\title{
Synthesis and Characterisation of Core-shell Gold Nanoparticles
}

by

Michelle Jane Cook

\author{
A thesis \\ submitted to Victoria University of Wellington \\ in partial fulfilment of the requirements for the degree of \\ Master of Science in Chemistry
}

Victoria University of Wellington 


\section{Abstract}

Technology developed at Victoria University of Wellington by Professor James H. Johnston and Dr Kerstin Lucas allows for the colouring of high quality wool fibres using spherical gold nanoparticles. Gold nanoparticles have interesting colours and optical properties due to surface plasmon resonance effects and, using this technology, a boutique range of colours can be imparted onto wool fibres. The colour of gold nanoparticles is determined by their size and shape, hence the colour range achievable using spherical nanoparticles is limited to those obtained by changing the particle diameter and degree of aggregation of these particles. This limitation can be overcome by using gold nanoparticles of different shapes in conjunction with other materials. This research details the synthesis and characterisation of gold nanoshells on spherical silica cores and their use for the colouring of wool. Silica cores were used in this research as they are reasonably chemically inert and so serve as a stable substrate for the gold shells. Silica spheres are also easily prepared in a manner that allows control over the final particle diameter.

Several syntheses of these core-shell particles have been previously devised however they are not suitable for commercial use. Such syntheses involve many timeconsuming steps, high temperatures or light-sensitive reagents. Synthetic methods set out in this research involve a novel in-situ seeding of gold nanoparticles for the growth of the shells eliminating the step of growing gold nanoparticles ex-situ commonly involved in other synthetic schemes. The need for light-sensitive reducing agents is eliminated by the use of other reductants such as sodium borohydride and hydroxylamine. All steps of the synthetic schemes are carried out at less than $100{ }^{\circ} \mathrm{C}$. Several methods of synthesising core-shell particles are outlined in this research, which achieved varying degrees of success. Many syntheses investigated successfully produced core-shell particles but also left many silica spheres without the desired gold shell coating. This was not a problem for the proposed application of colouring wool. As silica is easily dispersed in water and does not have the same affinity to bind to wool as gold does, the silica spheres without gold shells simply wash off after colouring. This allowed the core-shell particles synthesised in this research to be successfully used to colour wool fibres and achieve a shade of purple not previously obtained using the earlier methodologies. 


\section{Acknowledgements}

I would first like to thank my supervisor Professor Jim Johnston for all of his help and support throughout this research project. His guidance over the past two years has been incredibly valuable and I am very grateful for his time and thoughts.

Thanks also to everyone in the School of Chemical and Physical Sciences here at Victoria. Specific mention must go to David Flynn for his tireless help with the electron microscopes for which I am very appreciative. I would also like to thank all of the lecturing staff that contributed to the coursework to fulfil the other requirements of this degree.

To my lab group, past and present, including Dr Kerstin Lucas, Dr Mathew Cairns, Dr Maria Parry, Andrea Kolb, Thomas Nilsson, Matthias Herzog, and Eldon Tate I could not have survived this challenge without your help, and I am glad to have had the opportunity to work with such great, kind people.

A big thank you to everyone who helped proof read this thesis, Danielle Thorne, Struan Cummins and Thomas Nilsson.

I also thank Victoria University of Wellington for the Curtis-Gordon Research Scholarship in Chemistry for 2013, and again Professor Jim Johnston for providing me with an externally funded Masters Scholarship.

And finally, I would like to thank my family and friends for their constant loving support. 


\section{Contents}

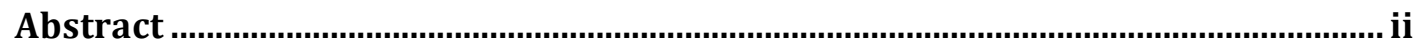

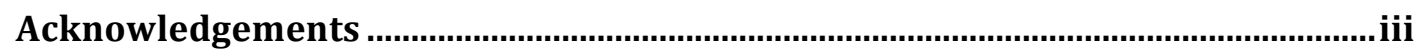

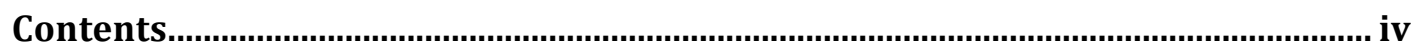

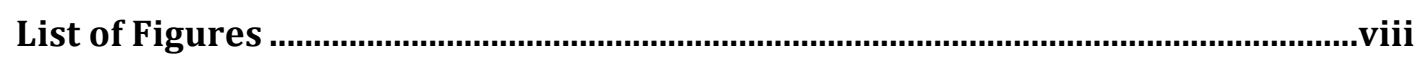

List of Tables...........................................................................................................

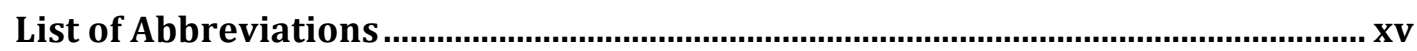

1. Introduction

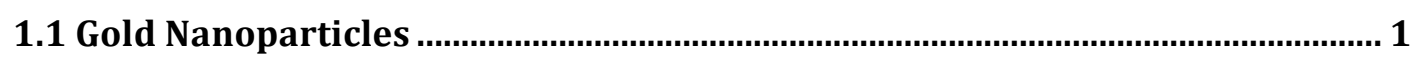

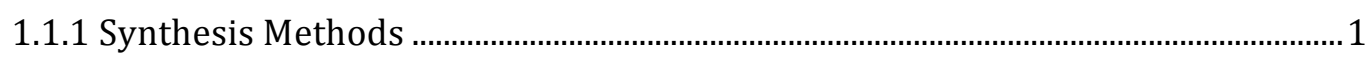

1.1.2 Applications of Gold Nanoparticles ......................................................................

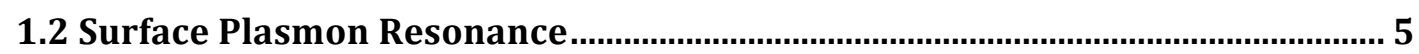

1.2.1 SPR of Spherical Particles .................................................................................................

1.2.2 SPR of Rod-shaped Particles .........................................................................................

1.2.3 SPR of Core-shell Particles........................................................................................ 11

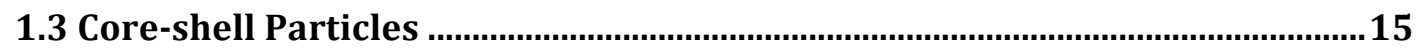

1.3.1 Gold Shells........................................................................................................... 15

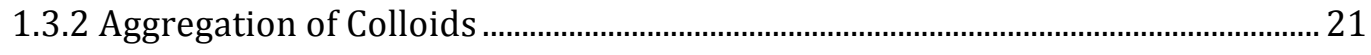

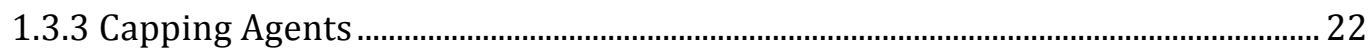

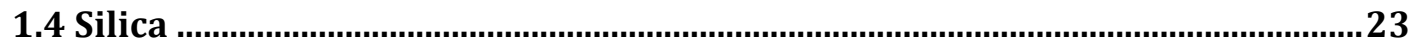

1.4.1 Stöber Process...................................................................................................... 24

1.5 Titania

1.6 Nanogold as a Colourant in Wool Fibres: An Application of SPR ......................28

1.7 Project Aims............................................................................................................

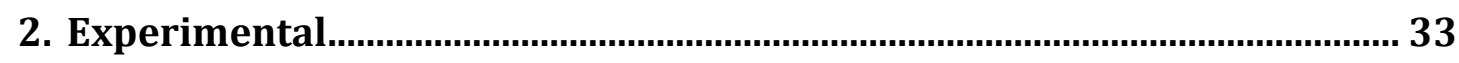

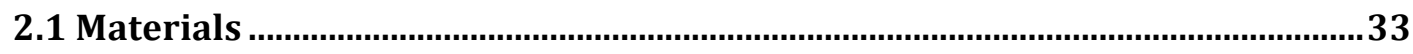

2.1.1 Purchased Materials ...................................................................................................... 33

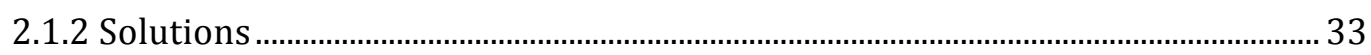

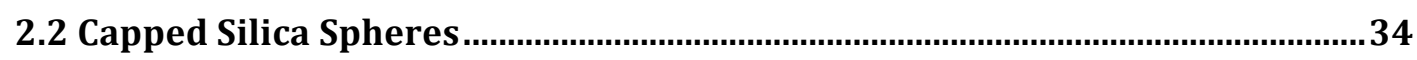

2.2.1 Silica Spheres...................................................................................................... 34

2.2.2 PEI Capped Silica Spheres ....................................................................................... 34

2.2.3 MPTMS Capped Silica Spheres............................................................................... 35

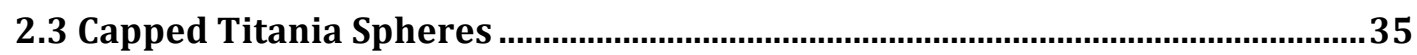

2.3.1 Titania Spheres Capped with MPTMS..................................................................... 35 
2.4 Gold Nanoshells on Capped Silica Spheres

2.4.1 Ex-situ Seeding on PEI Capped Silica Spheres......................................................... 35

2.4.2 In-situ Seeding on PEI Capped Silica Spheres................................................................ 36

2.4.3 In-situ Seeding on PEI Capped Silica Spheres with Hydroxylamine ..................... 36

2.4.4 In-situ Seeding on MPTMS Capped Silica Spheres.................................................... 36

2.4.5 In-situ Seeding on MPTMS Capped Silica Spheres with Hydroxylamine ........... 37

2.4.6 In-situ Seeding on MPTMS Capped Silica Spheres with $\mathrm{NaBH}_{4}$ and

Hydroxylamine ............................................................................................................. 37

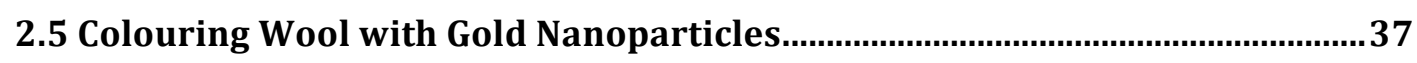

2.5.1 Spherical Gold Nanoparticles .................................................................................... 38

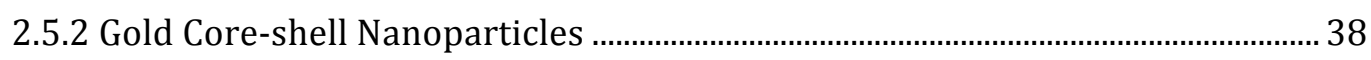

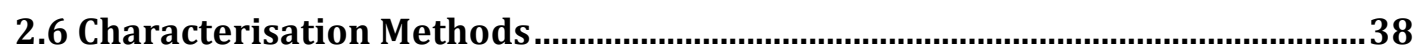

2.6.1 UV-Vis Absorption Spectroscopy ………………………………………........... 38

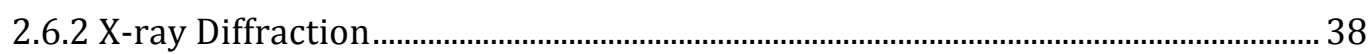

2.6.3 Scanning Electron Microscopy................................................................................... 38

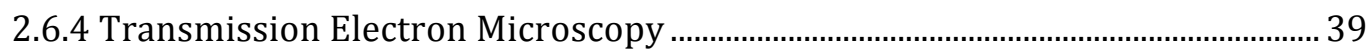

2.6.5 Size Measurements ................................................................................................. 39

2.6.6 Colour Measurements................................................................................................ 40

3. Characterisation of Silica and Titania ..................................................... 42

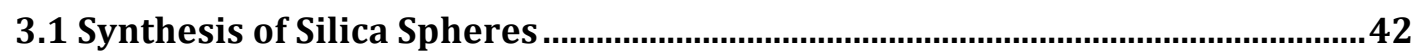

3.1.1 UV-Vis Absorption Spectroscopy ……………....................................................... 42

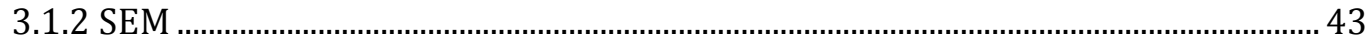

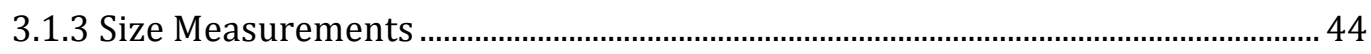

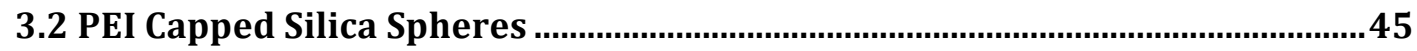

3.2.1 UV-Vis Absorption Spectroscopy ……………..................................................... 45

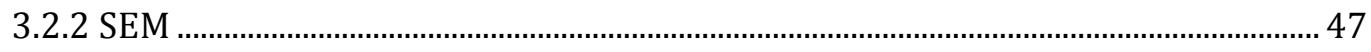

3.2.3 Size Measurements ................................................................................................ 49

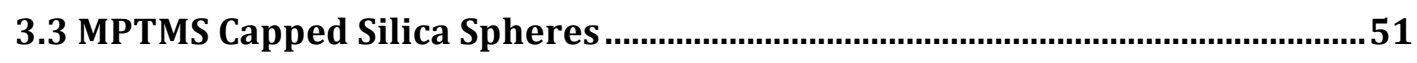

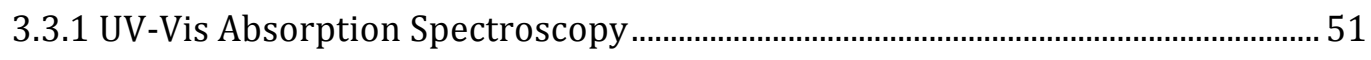

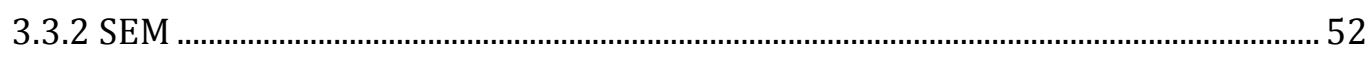

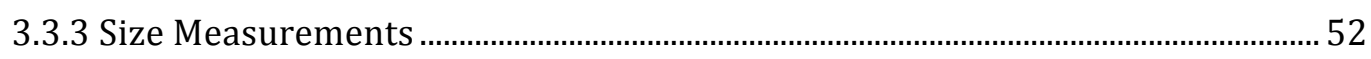

3.4 MPTMS Capped Titania Spheres …................................................................53

3.5 Conclusions from Characterisation of Silica and Titania ..................................53

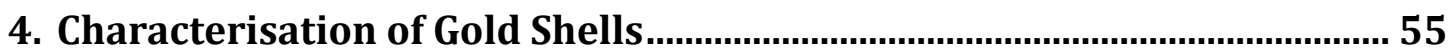

4.1 Ex-situ Seeding on PEI Capped Silica Spheres......................................................55 
4.1.1 UV-Vis Absorption Spectroscopy …………........................................................ 57

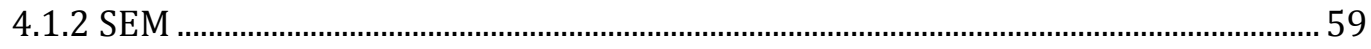

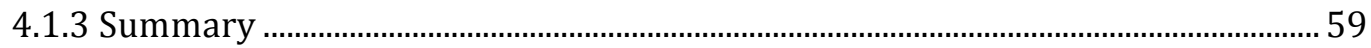

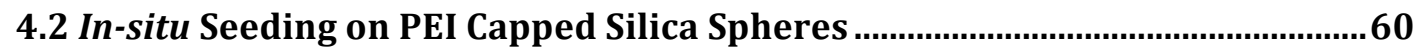

4.2.1 UV-Vis Absorption Spectroscopy …………............................................................ 62

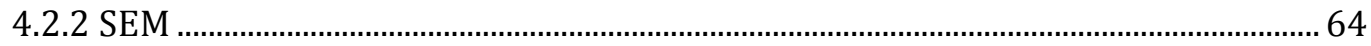

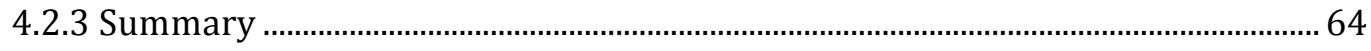

4.3 In-situ Seeding on PEI Capped Silica Spheres with Hydroxylamine..................65

4.3.1 UV-Vis Absorption Spectroscopy …………………………………………......... 65

4.4 In-situ Seeding on MPTMS Capped Silica Spheres.............................................66

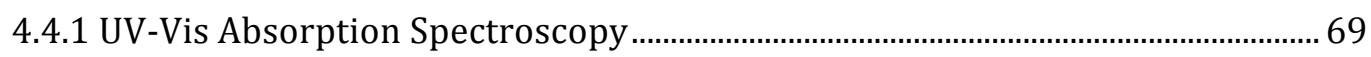

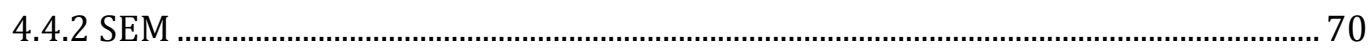

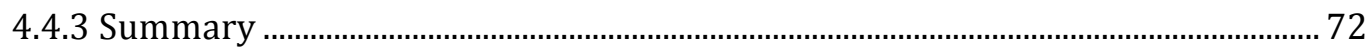

4.5 In-situ Seeding on MPTMS Capped Silica with Hydroxylamine ........................74

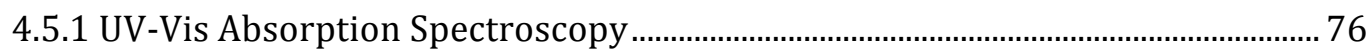

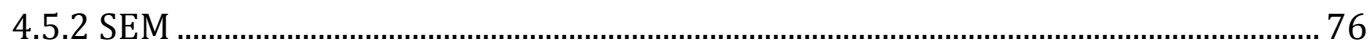

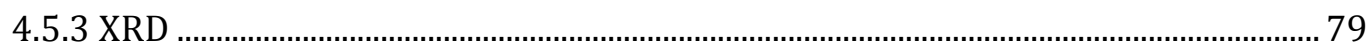

4.5.4 TEM

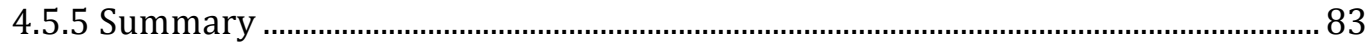

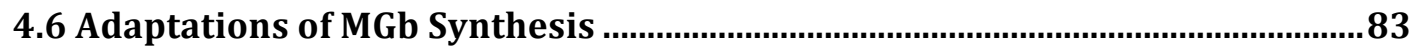

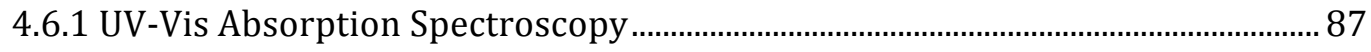

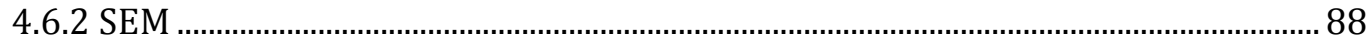

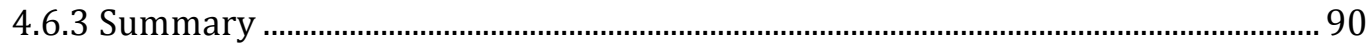

4.7 In-situ Seeding on MPTMS Capped Silica Spheres with $\mathrm{NaBH}_{4} \ldots \ldots . . . . . . . . . . . . . . . . . .91$

4.7.1 UV-Vis Absorption Spectroscopy ………………………………………….......... 92

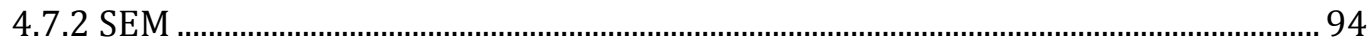

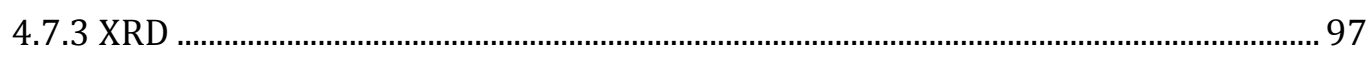

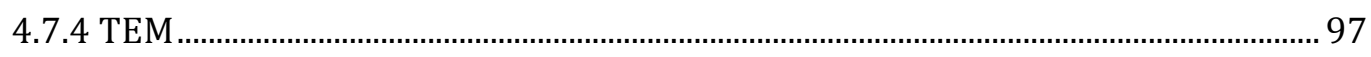

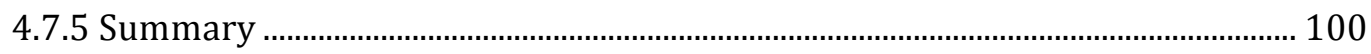

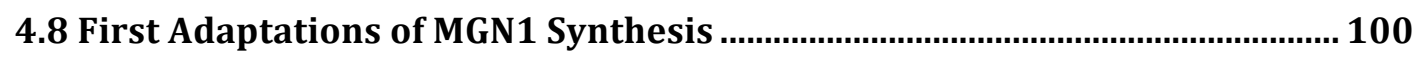

4.8.1 UV-Vis Absorption Spectroscopy …………........................................................... 102

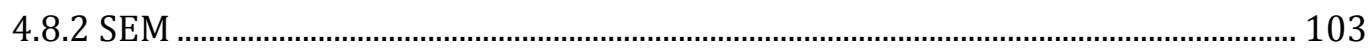

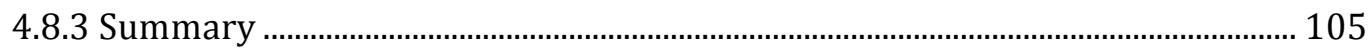

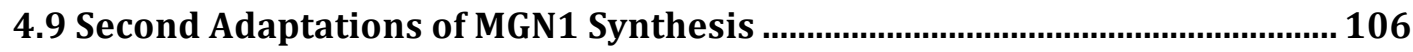

4.9.1 UV-Vis Absorption Spectroscopy ………………….............................................. 108

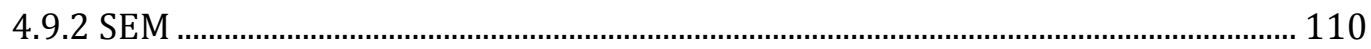


4.9.3 XRD

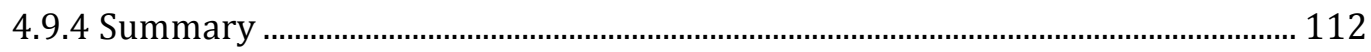

4.10 Conclusions from Silica-gold Particles ............................................................... 113

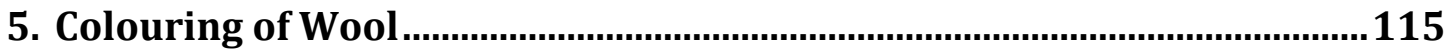

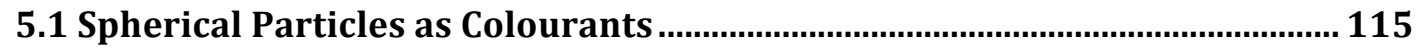

5.1.1 Colour Measurements........................................................................................... 115

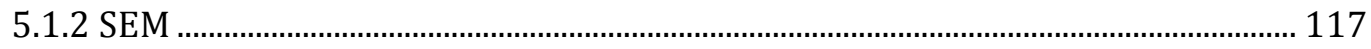

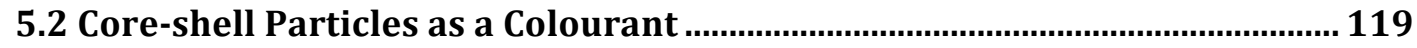

5.2.1 UV-Vis Absorption Spectroscopy ………………….............................................. 119

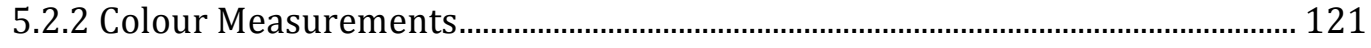

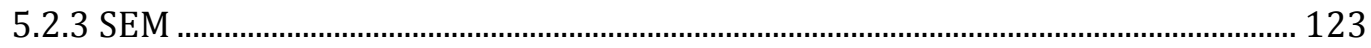

5.3 Conclusions from Colouring of Wool ................................................................ 125

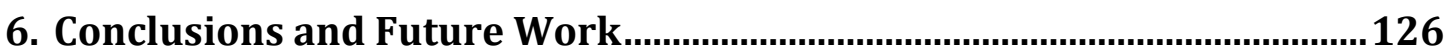

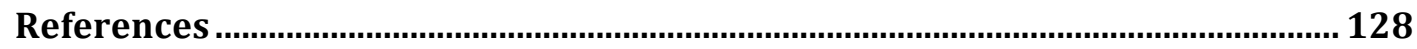




\section{List of Figures}

Figure 1.1: Pourbaix diagram of gold showing stability of $\mathrm{AuCl}_{4}{ }^{-}$complex. 2

Figure 1.2: Redox coupling of citric acid and tetrachloroaurate. 3

Figure 1.3: TEM image of gold nanoparticles synthesised using trisodium citrate...... 3

Figure 1.4: Turnover frequency for carbon monoxide oxidation for $\mathrm{Au} / \mathrm{TiO}_{2}$ as a function of the mean diameter of the particles at $273 \mathrm{~K}$. 5

Figure 1.5: The electric field of electromagnetic radiation interacts with the conduction band electrons of surface atoms, causing a wave of dipoles. 6

Figure 1.6 Electromagnetic radiation interactions with spherical and rod-shaped particles. 7

Figure 1.7: UV-Vis absorption spectra and TEM images of spherical gold nanoparticles, showing red shift of SPR absorption peak in larger diameter particles. 8

Figure 1.8: UV-Vis absorption spectra and TEM images of rod-shaped gold nanoparticles, showing both transverse and longitudinal SPR absorption peaks and their dependence on the aspect ratio of the particle. 10

Figure 1.9: Symmetric and anti-symmetric resonance modes of a metal nanoshell... 12

Figure 1.10: Schematic drawing of a core-shell particle showing core diameter and shell thickness. Theoretically calculated resonances of gold shells on spherical silica core particles showing the red shift of symmetric SPR absorption peak in particles with thicker shells. 13

Figure 1.11: Absorbance spectra of $\mathrm{AuS}_{2}-\mathrm{Au}$ core-shell particles predicted by Mie theory and gathered experimentally by Averitt et al. 14

Figure 1.12: TEM images of gold nanoparticles deposited onto silica core particles capped with APTMS forming large clusters with little interaction or capped with PEI forming very rough shells with large protrusions. 
Figure 1.13: TEM image of uncapped silica spheres with gold nanoparticle aggregates, TEM image of a uneven full gold shell on APTES capped silica sphere, UV-Vis absorption spectra of these full gold shell particles with varying shell to core volume ratios. 18

Figure 1.14: TEM images showing growth of gold nanoshells using K-gold solution after deposition of ex-situ synthesised gold nanoparticle seeds.

Figure 1.15: TEM images showing the formation of a gold shell on a polystyrene sphere capped with AET, UV-Vis absorption spectra of gold nanoparticles and gold decorated polystyrene spheres. 20

Figure 1.16: Schematic drawing demonstrating the factors involved in steric stabilisation of nanoparticles in a colloid due to the unfavourable interactions between adsorbed surfactant molecules. 22

Figure 1.17: EM image of silica spheres synthesised via the Stöber method. 24

Figure 1.18: Graph showing dependence of particle size on reagent concentrations in Stöber process of synthesising silica spheres.

Figure 1.19: UV-Vis of samples prepared by Pandikumar et al. with varying molar ratios of $\mathrm{TiO}_{2}$ :Au showing absorbance at approximately $520 \mathrm{~nm}$. TEM image of titania and gold particles synthesised by Pandikumar et al.

Figure 1.20: A selection of colours achieved using gold nanoparticles as colourant in wool. 30

Figure 2.1: CIE Lab colour space. 40

Figure 3.1: UV-Vis absorption spectrum of prepared silica spheres. 43

Figure 3.2: SEM image of synthesised silica particles showing size and monodispersity. 44

Figure 3.3: Size distribution of particles in prepared silica colloid showing average of $186 \mathrm{~nm}$ and good monodispersity. 45

Figure 3.4: UV-Vis absorption spectra of PEI capped silica spheres. 46 
Figure 3.5: UV-Vis absorption spectrum of a $5 \mathrm{wt} \%$ PEI solution in water. 46

Figure 3.6: SEM images of PEI capped silica spheres in both SEI and backscatter modes. 48

Figure 3.7: Size distributions of prepared PEI capped silica sphere colloids showing polydispersity and average particle size. 50

Figure 3.8: UV-Vis absorption spectrum of MPTMS capped silica spheres. 51

Figure 3.9: SEM images of silica spheres capped with MPTMS in both SEI and backscatter modes. 52

Figure 3.10: Size distribution of prepared MPTMS capped silica colloid showing monodispersity and average particle size.......

Figure 4.1: UV-Vis absorption spectra of ex-situ seeded gold shells on PEI capped silica spheres. The samples were not the same concentrations. 58

Figure 4.2: SEM images of Sample 10-A01 in both SEI and backscatter modes. 59

Figure 4.3: Photograph of samples 10-C01, 10-C02, 10-C01a and 10-C02a. 62

Figure 4.4: UV-Vis absorption spectra of samples prepared via in-situ seeding on PEI capped silica spheres. 63

Figure 4.5: SEM images of sample 10-C01 in both SEI and backscatter modes. 64

Figure 4.6: UV-Vis absorption spectrum of PGb showing no absorption peak in the visible region. 66

Figure 4.7: Photograph of samples MG98-1, MG98-2, MG98-3 and MG75. 68

Figure 4.8: Photographs of sample MG98-1, demonstrating different observed colours in reflected and transmitted light. 68

Figure 4.9: UV-Vis absorption spectra of samples prepared via in-situ seeding on MPTMS capped silica spheres. 70 
Figure 4.10: SEM images of sample MG98-3 showing large core-shell particles and of sample MG98-2 showing gold aggregated with silica spheres.

Figure 4.11: SEM images of sample MG75 in both SEI and backscatter modes showing small spherical gold particles, gold platelets and gold aggregates....... 71

Figure 4.12: EDS mapping of MG75 showing oxygen signal in the same areas as gold spheres

Figure 4.13: Photograph of samples MG, MGa and MGb. 75

Figure 4.14: UV-Vis absorption spectra of $\mathrm{MG}, \mathrm{MGa}$ and $\mathrm{MGb}$, prepared via in-situ seeding on MPTMS capped silica with hydroxylamine. Samples were not of equal concentrations. 76

Figure 4.15: SEM images of MG and MGa in both SEI and backscatter mode. 77

Figure 4.16: SEM images of MGb in both SEI and backscatter modes. 78

Figure 4.17: XRD spectrum of sample MGa showing peaks due to crystal planes of gold metal. 79

Figure 4.18: XRD spectrum of sample MGb showing peaks due to crystal planes of gold metal. 80

Figure 4.19: TEM image of MGb showing small gold nanoparticles and a silica sphere. 81

Figure 4.20: STEM EDS images of MGb showing large area of gold associated with silica spheres. 82

Figure 4.21: Photograph of samples MGb-a, -b, -c, -d, -e, -f, -g. 85

Figure 4.22: UV-Vis absorption spectra of samples prepared via the adaptation of MGb synthesis, all showing peaks at approximately $710 \mathrm{~nm}$. 87

Figure 4.23: UV-Vis absorption spectra of MGb-e and MGb-f prepared via adaptations of MGb synthesis showing peaks at approximately $525 \mathrm{~nm}$, deemed to be due to the presence of spherical gold nanoparticles in the samples. 88 
Figure 4.24: SEM image of MGb-c in backscatter mode representative of samples MGb-b, MGb-c, MGb-e and MGb-g, showing triangular and hexagonal platelets of high-density material.

Figure 4.25: EDS mapping of MGb-f showing silica-gold core-shell particle. 90

Figure 4.26: UV-Vis absorption spectra of samples MGN1, MGN2 and MGN3, synthesised via in-situ seeding on MPTMS capped silica spheres with $\mathrm{NaBH}_{4}$. Samples were not of equal concentration. 94

Figure 4.27: SEM images of MGN1 in both SEI and backscatter modes, showing smooth spheres of high-density material, of approximately the size of silica spheres. 95

Figure 4.28: SEM images of MGN2 in both SEI and backscatter modes. 95

Figure 4.29: EDS mapping of MGN1 showing silica-gold core-shell particle. 96

Figure 4.30: XRD spectrum of sample MGN1 showing peaks due to crystal planes of gold metal. 97

Figure 4.31: TEM images of large dense particles in sample MGN1, showing almost spherical shape. Silica spheres without gold shells are also seen in the image on the right. 98

Figure 4.32: STEM EDS maps of dense particles in sample MGN1, showing presence of silicon and oxygen with gold. 99

Figure 4.33: UV-Vis absorption spectra of samples prepared by first adaptations of MGN1 synthesis. 103

Figure 4.34: SEM images of MGN1-1a and MGN1-1W in SEI mode, showing large blobby areas of gold on silica sphere aggregates. 104

Figure 4.35: SEM images of MGN1-1b in both SEI and backscatter modes, showing particles that look like core-shells 104

Figure 4.36: EDS map of MGN1-1b showing silica-gold core-shell particle. 105 
Figure 4.38: UV-Vis absorption spectra of samples prepared by second adaptations of MGN1 synthesis.

Figure 4.39: SEM images of sample MGN1b in both SEI and backscatter modes, showing several core-shell particles. 110

Figure 4.40: EDS mapping of sample MGN1c, showing presence of oxygen in the areas deemed to be core-shell particles.

Figure 4.41: XRD spectrum of sample MGN1b showing peaks due to crystal planes of gold metal. 112

Figure 5.1: Photographs of wool samples coloured pink, purple and grey with spherical gold nanoparticles.

Figure 5.2: Kubelka-Munk transformed reflectance spectra of uncoloured wool and wools coloured with spherical gold nanoparticles. 116

Figure 5.3: SEM images of wool coloured pink, purple and grey by spherical gold nanoparticles. 118

Figure 5.4: Photograph of wool coloured using silica-gold core-shell particles. 119

Figure 5.5: UV-Vis absorption spectrum of liquid left over after colouring wool... 120

Figure 5.6: UV-Vis absorption spectrum of MGN1c before colouring wool. 121

Figure 5.7: Kubelka-Munk transformed reflectance spectrum of wool coloured with core-shell nanoparticles, compared to spectra of wool coloured with spherical gold nanoparticles. 122

Figure 5.8: SEM images in both SEI and backscatter modes of wool coloured with silica-gold core-shell particles. 124

Figure 5.9: EDS map of wool coloured with silica-gold core-shell particles. 125 


\section{List of Tables}

Table 1.1: Sulfur containing amino acids in wool.

Table 3.1: Size measurements of diameters of silica spheres capped with PEI 49

Table 4.1: Samples prepared via ex-situ seeding on PEI capped silica spheres. 56

Table 4.2: Samples prepared via in-situ seeding on PEI capped silica spheres. 61

Table 4.3: Samples prepared via in-situ seeding on MPTMS capped silica particles. 67

Table 4.4: Wavelengths of major and minor absorption bands of samples prepared via in-situ seeding on MPTMS capped silica spheres. 69

Table 4.5: Samples prepared via in-situ seeding on MPTMS capped silica spheres. 74

Table 4.6: Adaptations of MGb synthesis. 86

Table 4.7: Samples prepared via in-situ seeding on MPTMS capped silica spheres with $\mathrm{NaBH}_{4}$ 92

Table 4.8: Samples prepared via adaptation of MGN1 synthesis. 102

Table 4.9: Samples prepared by second adaptations of original MGN1 synthesis... 107

Table 4.10: Wavelengths of major absorption peaks in UV-Vis absorption spectra of samples prepared by second adaptations of MGN1 synthesis. 109

Table 5.1: CIE Lab values for uncoloured wool and wool coloured pink, purple and grey using spherical gold nanoparticles.

Table 5.2: CIE Lab values of uncoloured wool, wool coloured with spherical gold nanoparticles and wool coloured with silica-gold core-shell particles. 


\section{List of Abbreviations}

AET: 2-aminoethanethiol

APTES: (3-aminopropyl)-triethoxysilane

APTMS: (3-aminopropyl)-trimethoxysilane

EDS: $\quad$ Energy dispersive X-ray spectroscopy

MO: Molecular orbital

MPTMS: (3-mercaptopropyl)-trimethoxysilane

PEI: $\quad$ Polyethyleneimine

SEM: $\quad$ Scanning electron microscopy

SPR: $\quad$ Surface plasmon resonance

STEM: Scanning transmission electron microscopy

TA: $\quad$ Tannic acid

TEOS: Tetraethylorthosilicate

TEM: Transmission electron microscopy

TMOS: Tetramethylorthosilicate

TSC: $\quad$ Trisodium citrate

UV-Vis: Ultra-violet visible

XRD: X-ray Diffraction 


\section{Introduction}

\subsection{Gold Nanoparticles}

Gold nanoparticles have been used for hundreds of years. A common early use of gold nanoparticles was to impart red colouring in stained glass for windows in grand buildings such as cathedrals. Although the optical properties of gold nanoparticles have been exploited for centuries, the understanding of this phenomenon was not gained until much later. In 1857 Michael Faraday prepared the first pure sample of colloidal gold by reducing gold chloride with phosphorus. This led to the understanding that the ruby red colour was due to gold but the mechanism was still a mystery. In 1908 Gustav Mie solved Maxwell's equations for describing the scattering of electromagnetic radiation by a sphere, and it was found that particles of sizes on the scale of the wavelength of light behaved in an interesting manner causing them to appear coloured (see Section 1.2 Surface Plasmon Resonance). ${ }^{1}$

\subsubsection{Synthesis Methods}

Gold metal has low reactivity and so a more reactive gold compound must be used in chemical reactions. A solution of $\mathrm{AuCl}_{4}{ }^{-}$in acidic conditions can be prepared by dissolving gold metal in aqua regia (3:1 solution of concentrated nitric and hydrochloric acids) then removing any excess nitrate as nitrogen by reaction with urea. This solution is a desirable starting material for gold reactions as it is stable over a wide range of $\mathrm{pH}$ levels. As shown by Figure 1.1, some gold complexes are very sensitive to $\mathrm{pH}$ and can easily form insoluble $\mathrm{Au}(\mathrm{OH})_{3}$. For this reason, tetrachloroaurate is a common starting material for gold nanoparticle syntheses. 


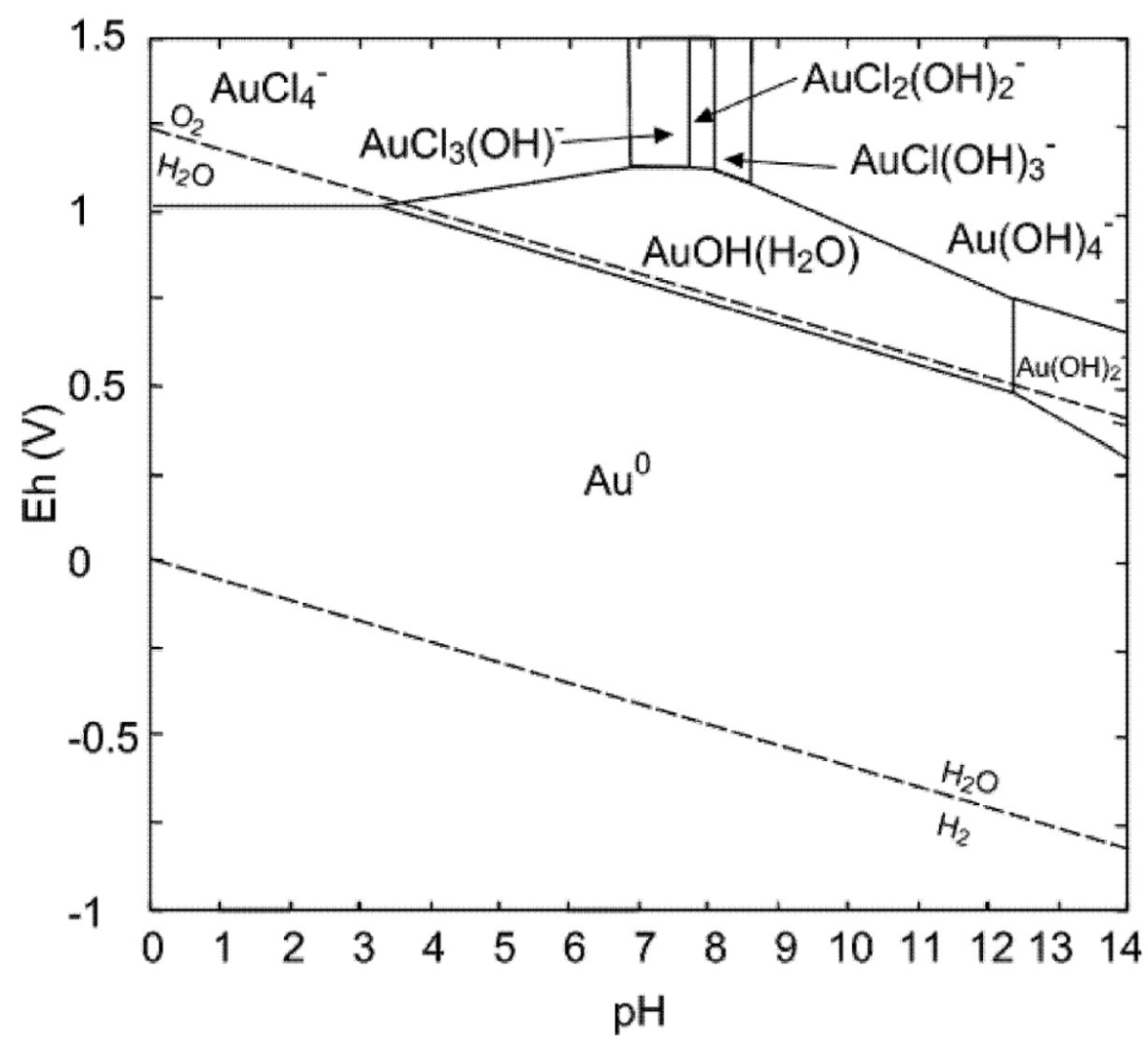

Figure 1.1: Pourbaix diagram of gold showing stability of $\mathrm{AuCl}_{4}^{-}$complex.

Synthesis of gold nanoparticles requires a reducing agent to reduce $\mathrm{AuCl}_{4}{ }^{-}$to $\mathrm{Au}^{0}$ and a stabilising agent to prevent aggregation of the resulting nanoparticles in order to obtain a monodisperse gold nanoparticle colloid. The simplest and most commonly used synthesis of gold nanoparticles was first developed by Turkevich and colleagues in 1951 and involves the use of trisodium citrate (TSC) as both a reductant and stabiliser. ${ }^{2}$ As shown in Figure 1.2, when added to the acidic tetrachloroaurate solution the trisodium citrate forms citric acid, which is the reactive species. The trisodium citrate is added to a boiling aqueous solution of $\mathrm{HAuCl}_{4}$ under stirring and the solution soon develops a ruby red colour, characteristic of gold nanoparticles, after passing through a deep blue colour. Recently, this deep blue colour has been attributed to a transient intermediate of gold nanowires. ${ }^{3}$ A TEM image of gold nanoparticles synthesised via this method is shown in Figure 1.3. 


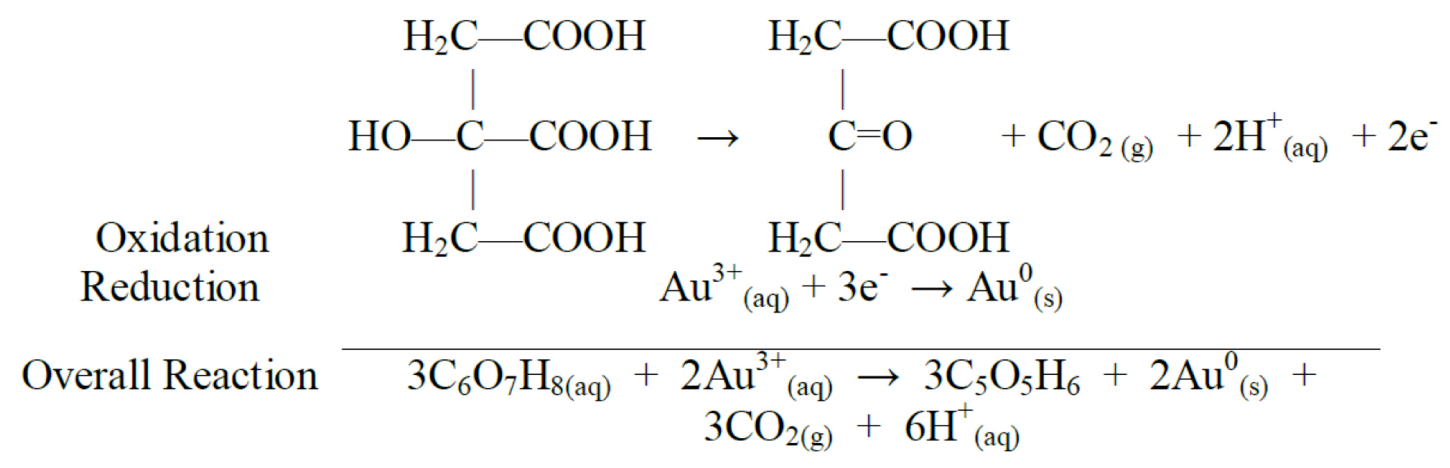

Figure 1.2: Redox coupling of citric acid and tetrachloroaurate. ${ }^{2}$

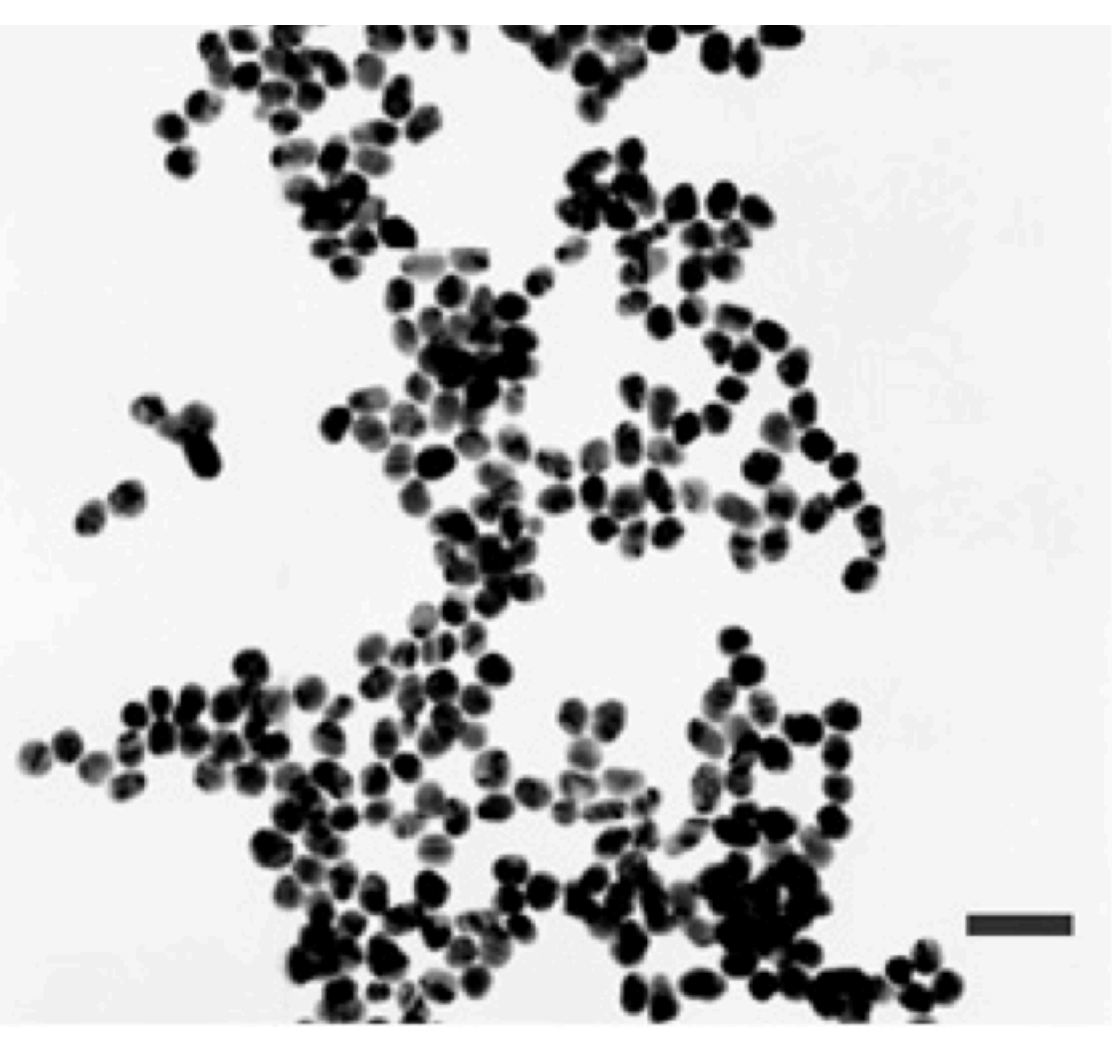

Figure 1.3: TEM image of gold nanoparticles synthesised using trisodium citrate. Scale bar is $100 \mathrm{~nm}^{4}$ 


\subsubsection{Applications of Gold Nanoparticles}

Gold is the least reactive metal and for this reason was long regarded as a poor heterogeneous catalyst. ${ }^{6}$ However, this notion was based on bulk gold, and it has since been found that gold nanoparticles can play an important part in catalysis reactions, including those for the low temperature oxidation of hydrogen and of carbon monoxide developed by M. Haruta and co-workers. ${ }^{7}$ A heterogeneous catalyst for these reactions can be formed by coprecipitation. This method involves adding an aqueous solution of tetrachloroaurate and a transition metal nitrate to an aqueous solution of sodium carbonate, followed by calcination of the coprecipitates. This produces gold nanoparticles of less than $10 \mathrm{~nm}$ diameter uniformly dispersed on the transition metal oxide. The transition metal oxides of iron, cobalt and nickel in conjunction with gold nanoparticles were highly active for both the oxidation of hydrogen and the oxidation of carbon monoxide. The catalytic activity of gold nanoparticles on transition metal oxide supports is heavily dependent on the mean diameter of the gold nanoparticles. It has been shown by Haruta and co-workers that the turnover frequency of these catalysts increases substantially with mean particle diameters of less than $5 \mathrm{~nm}$, as demonstrated by Figure 1.4. ${ }^{6-8}$ 


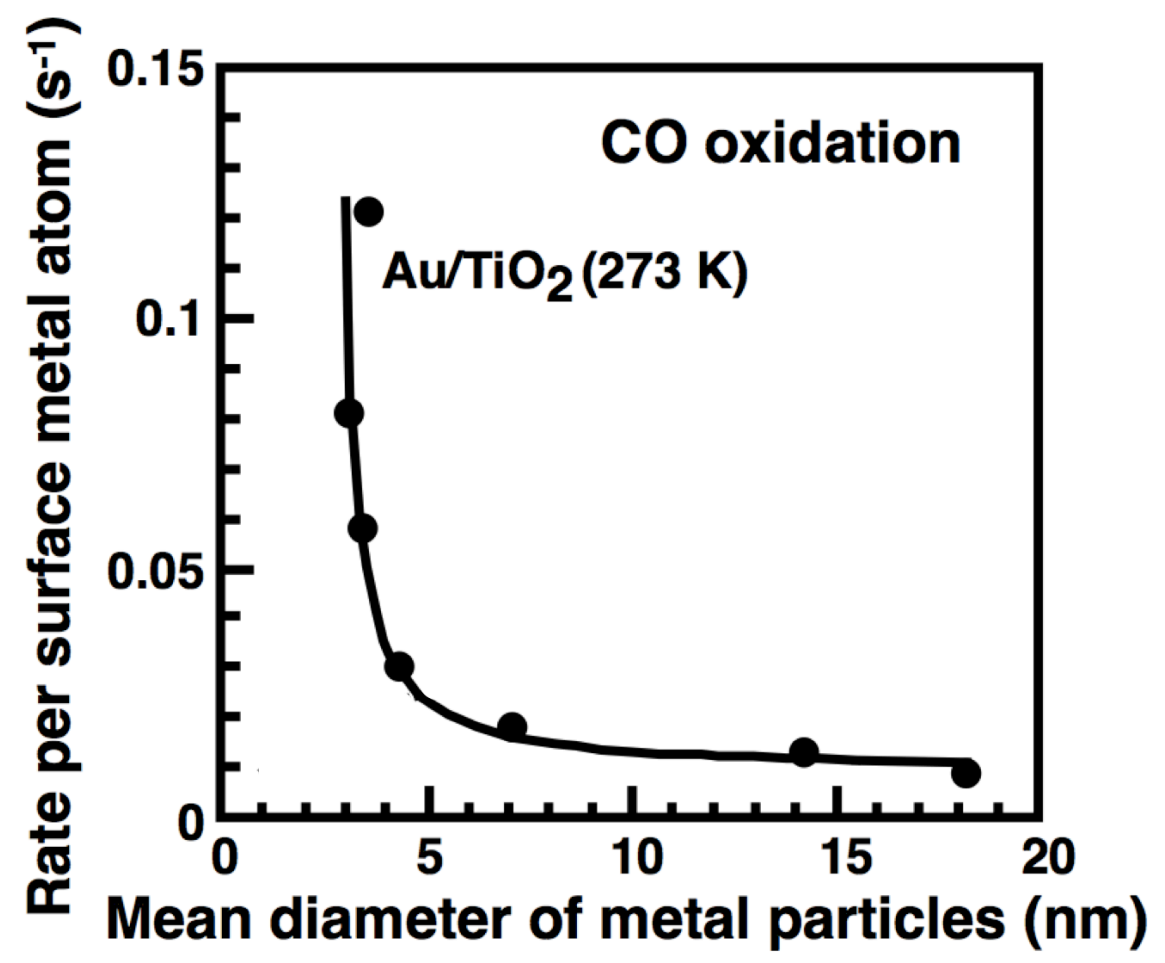

Figure 1.4: Turnover frequency for carbon monoxide oxidation for $\mathrm{Au} / \mathrm{TiO}_{2}$ as a function of the mean diameter of the particles at $273 \mathrm{~K}^{9}$

\subsection{Surface Plasmon Resonance}

Surface plasmon resonance (SPR) is a phenomenon that occurs in metal nanoparticles. The phenomenon has been used for several hundreds of years in applications such as the colouring of stained glass windows, but the underlying science has only been understood since last century. SPR is caused by electromagnetic radiation interacting with the electrons in the conduction band of metal atoms at the surface of a particle. When a metal nanoparticle is subjected to electromagnetic radiation, the electric field induces dipoles in the conduction band electrons. A wave of dipoles is then created across the atoms at the surface of the particle, which oscillates in phase with the electric field of the electromagnetic radiation. $^{10,11}$ These surface waves (surface plasmons) resonate at a specific wavelength, and light of that wavelength is absorbed by the particle causing it to 
appear coloured. This intense absorption, in the UV to IR region is the basis for many applications of SPR active particles. ${ }^{11}$

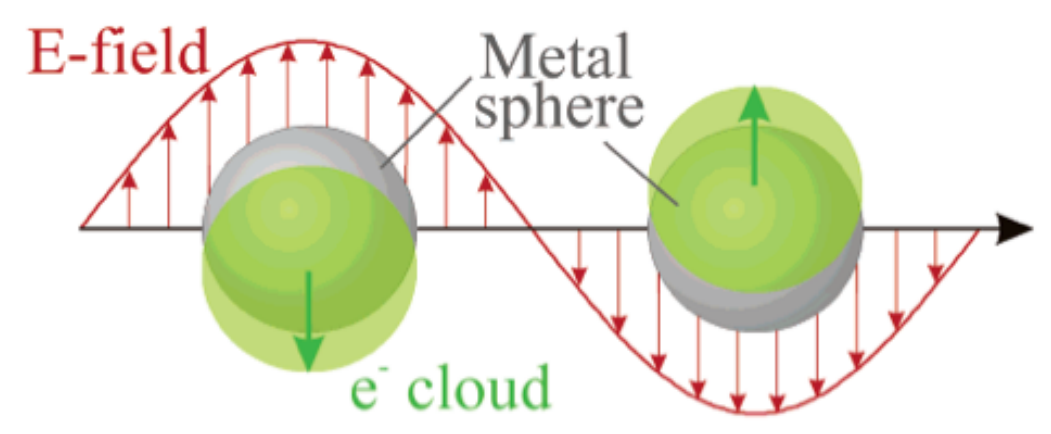

Figure 1.5: The electric field of electromagnetic radiation interacts with the conduction band electrons of surface atoms, causing a wave of dipoles. ${ }^{10}$

While SPR occurs in many metals, some metals such as aluminium and alkali earth metals have an oxide surface in air and do not appear coloured unless used in very inert atmospheres. Silver and gold are the most commonly used elements for SPR applications, as their SPR effects are the strongest in the visible region of the spectrum. ${ }^{11}$ However, as silver is more reactive than gold, SPR active silver nanoparticles will develop a thin oxide layer and the SPR absorption will be dampened and red shifted. ${ }^{12}$

SPR is a phenomenon that occurs at the surface of particles and is therefore sensitive to the dielectric constant of both the surrounding medium and the metal itself, as well as the shape and size of the particle. The intensity and wavelength of SPR absorptions are dependent on the surrounding medium as it affects both the incident light on the metal particle and the light reflected by the metal particle. A surrounding medium with a higher dielectric constant causes SPR absorption to occur at higher wavelengths. The dielectric constant, shape and size of the particle also affect the SPR absorption as the particle interacts differently with the incident electromagnetic radiation depending on these properties. ${ }^{11}$ 

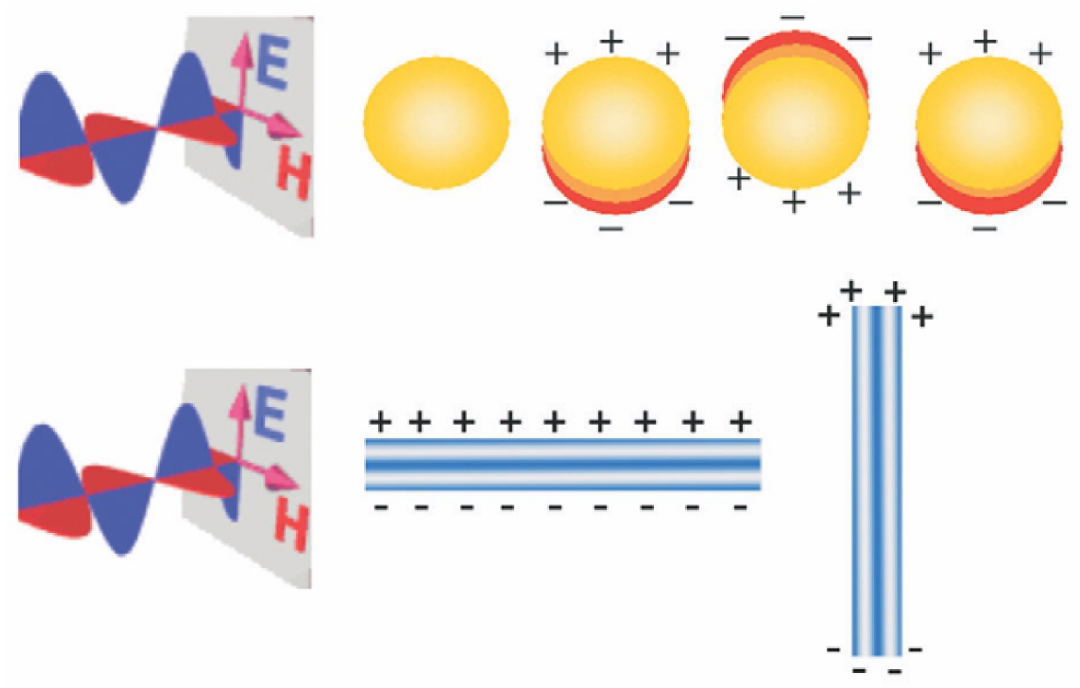

Figure 1.6 Electromagnetic radiation interactions with a) spherical and b) rodshaped particles. ${ }^{11}$

The effects of spherical particles on their SPR absorption can be explained by Mie theory. Equation $1.1^{11}$ shows the relationship between the extinction coefficient of the metal $\left(\mathrm{C}_{\mathrm{ext}}\right)$ and the radius of the particle $(\mathrm{R})$ depending on the wavelength of light $(\lambda)$, the dielectric constant of the metal ( $\varepsilon$ where $\left.\varepsilon=\varepsilon^{\prime}+\varepsilon^{\prime \prime}\right)$ and the dielectric constant of the surrounding medium $\left(\varepsilon_{\mathrm{m}}\right)$. As shown by the denominator of Equation 1.1, the extinction coefficient is highest when $\varepsilon^{\prime}=-2 \varepsilon_{\mathrm{m}}$ which correlates to when an absorption peak is observed. ${ }^{11}$

$$
C_{e x t}=\frac{24 \pi^{2} R^{3} \varepsilon_{m}^{3 / 2}}{\lambda} \frac{\varepsilon^{\prime \prime}}{\left(\varepsilon^{\prime}+2 \varepsilon_{m}\right)^{2}+\varepsilon^{\prime \prime 2}}
$$

Equation 1.1: Mie theory of SPR absorption in spherical particles. ${ }^{11}$

\subsubsection{SPR of Spherical Particles}

Spherical particles have only one SPR absorption peak as they have only one size dimension. The wavelength at which this absorption occurs depends on this size. 
That is to say, the SPR absorption wavelength of a spherical particle is determined by the diameter of the sphere, for a particular metal and surrounding medium. ${ }^{11}$ As demonstrated by Figure 1.7, the relationship between spherical particle diameter and SPR absorption wavelength is proportional, meaning a particle with a large diameter will have a high wavelength SPR absorption. The smallest particles shown in Figure 1.7 will appear pink whereas the larger particles will appear more purple as the particle diameter increases.
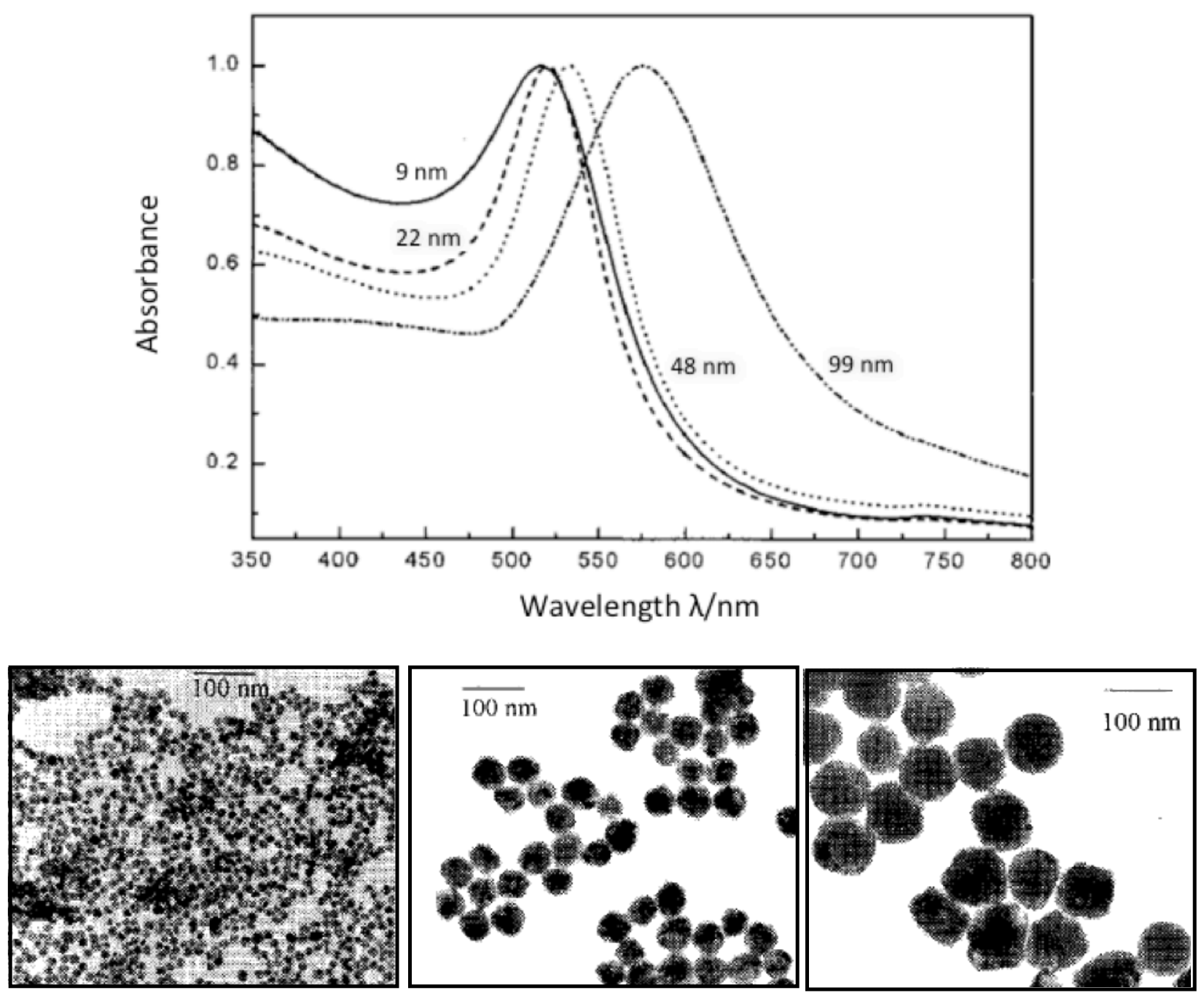

Figure 1.7: UV-Vis absorption spectra and TEM images of spherical gold nanoparticles, showing red shift of SPR absorption peak in larger diameter particles (TEM images: average size of gold nanoparticles, Left: $15 \mathrm{~nm}$, Middle: $48 \mathrm{~nm}$, Right: $99 \mathrm{~nm}){ }^{13}$ 


\subsubsection{SPR of Rod-shaped Particles}

Rod-shaped particles have two size dimensions and consequently have two SPR absorption peaks. One peak occurs at a wavelength controlled by the length-wise interaction of the particle with light, called the longitudinal peak. Conversely, the second peak occurs at a wavelength controlled by the width-wise interaction of the particle with light, called the transverse peak. ${ }^{11}$

Therefore, the observed colour of SPR active rod-shaped particles is dependent on the aspect ratio of the particle, i.e. the length of the particle relative to the width, for a particular metal and surrounding medium. This is demonstrated in Figure 1.8. The two SPR absorption peaks of short, fat rod-like particles with low aspect ratio (Sample A in Figure 1.8), can sometimes overlap, or appear at very similar wavelengths. This is intuitive as short, fat rod-like particles are closer to spherical and hence their SPR absorption characteristics are also close to spherical. By contrast, for long, thin rod-shaped particles with high aspect ratio (Sample E in Figure 1.8), the longitudinal peak occurs at higher wavelengths and with greater intensity than the transverse peak. The absorption spectra of low aspect ratio particles are more similar to those of spherical particles as the dimensions of the particle are closer to being spherical than high aspect ratio rod-shaped particles. ${ }^{14}$ 

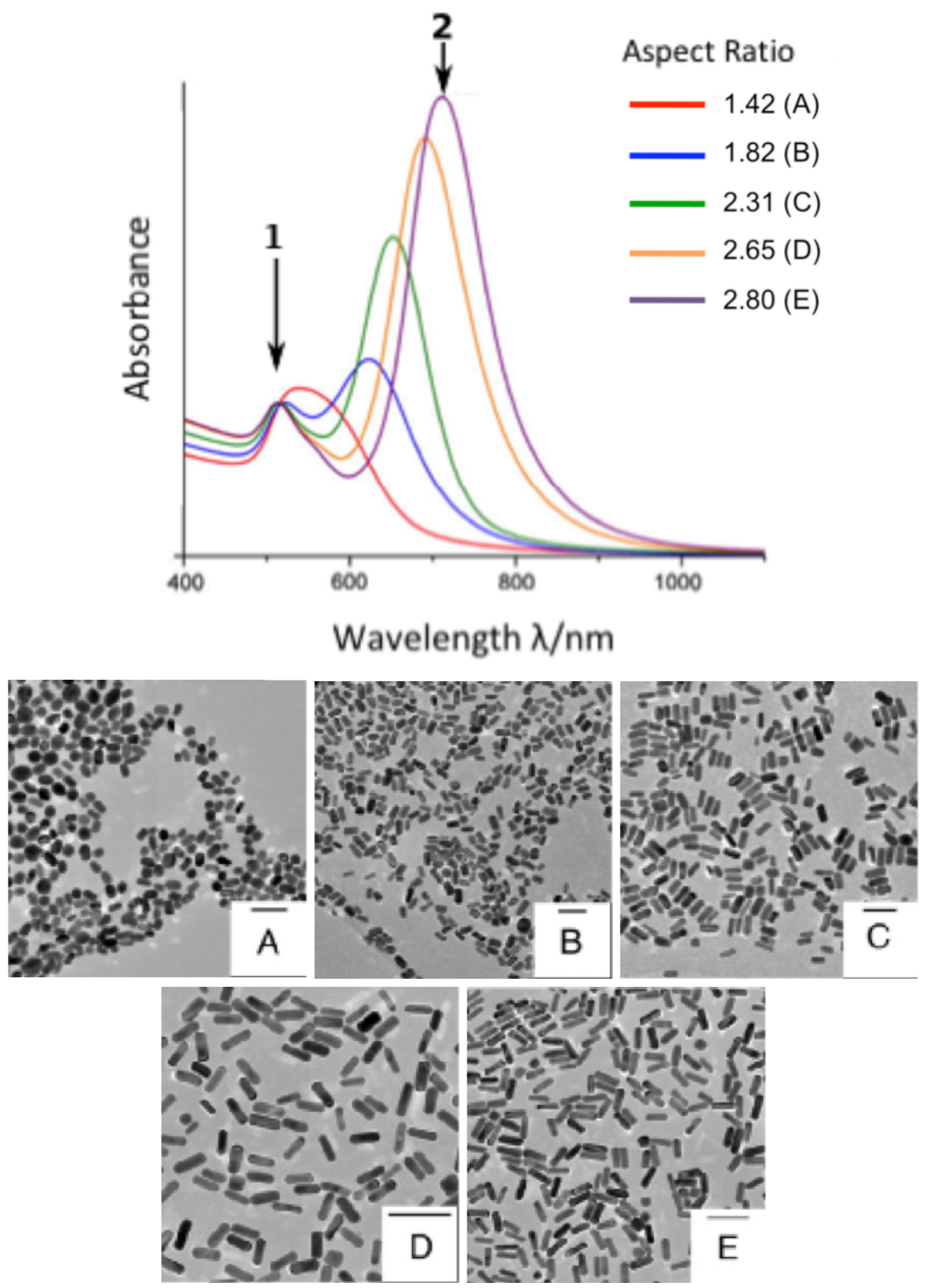

Figure 1.8: UV-Vis absorption spectra and TEM images of rod-shaped gold nanoparticles, showing both transverse (1) and longitudinal (2) SPR absorption peaks and their dependence on the aspect ratio of the particle (TEM images showing increasing aspect ratio from $A$ to $E) .^{14}$ 


\subsubsection{SPR of Core-shell Particles}

Core-shell particles are particles comprised of a spherical core of one material with an encompassing shell of another material (see Section 1.3 Core-shell Particles). SPR active metal shells have two SPR absorption peaks, similar to rod-shaped particles. However, while the two peaks of rod-shaped particles arise from coupling of interactions on two size dimensions, the two peaks of core-shell particles arise from the coupling of interactions of different materials. In core-shell particles, one interaction arises from the interface between the metal and the surrounding medium, while the other interaction arises from the interface between the metal and the core material. These interactions are hybridised in a way similar to molecular orbital (MO) theory. Instead of creating hybridised bonding and anti-bonding orbitals as in MO theory, the two interactions create hybridised symmetric and anti-symmetric resonance modes. ${ }^{15}$ These two resonance modes subsequently give rise to two SPR absorption peaks. The coupling of these two interactions and hence the SPR absorption wavelengths are determined by the distance between the surface and the core, therefore the observed colour of the particle is determined by the thickness of the shell relative to the total particle diameter. ${ }^{16}$ The thickness of the shell relative to the total particle diameter of a core-shell particle is commonly represented by the shell to core volume ratio. However, the anti-symmetric absorption mode is not significantly altered by size and lies near the bulk plasma frequency of the metal. The colour and optical properties of core-shell particles are determined by the symmetric absorption mode, so the anti-symmetric mode will not be investigated, as it is not relevant to this research. 

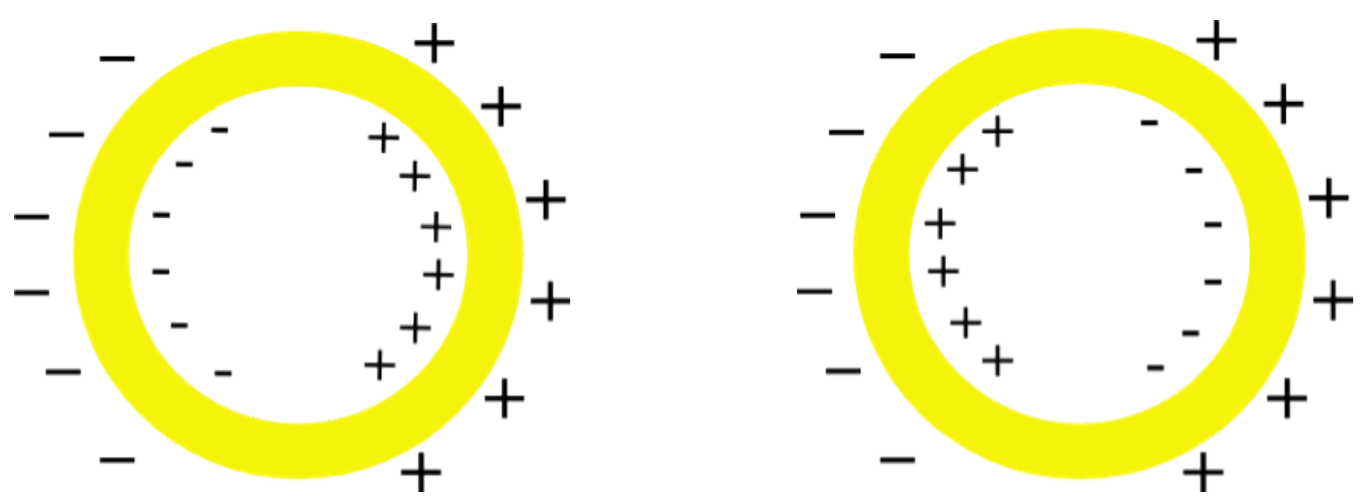

Figure 1.9: Symmetric (left) and anti-symmetric (right) resonance modes of a metal nanoshell. ${ }^{15}$

As shown in Figure 1.10, higher shell to core volume ratio causes the symmetric SPR absorption peak of the particle to blue shift and occur at shorter wavelengths. ${ }^{17,18}$ The low wavelength shoulder peak in the UV-Vis absorption spectra of thicker nanoshells is induced by the thickness of the shell and corresponds to a higher order SPR mode. The dependence of absorption characteristics on shell thickness allows for core-shell particles to be tailored to provide different optical properties, useful in many popular applications of core-shell particles (see Section 1.3 Core-shell Particles). 

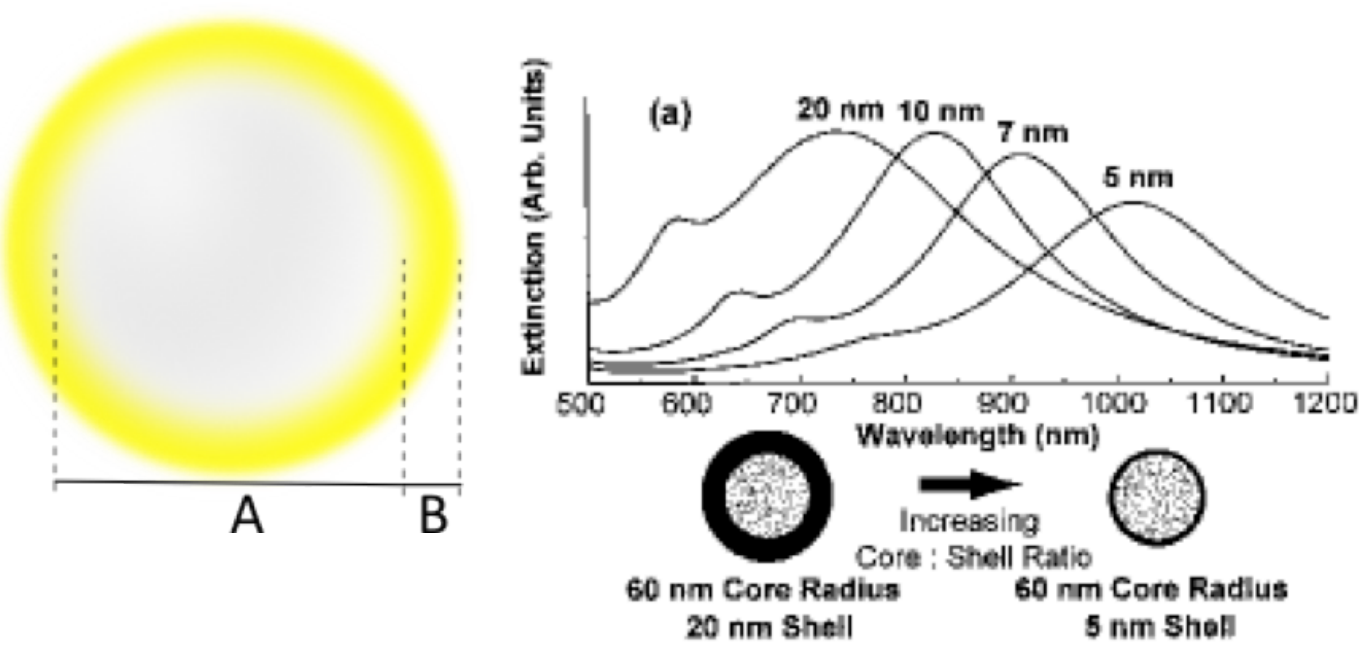

Figure 1.10: Left: Schematic drawing of a core-shell particle showing core diameter (A) and shell thickness (B), Right: Theoretically calculated resonances of gold shells on spherical silica core particles showing the red shift of symmetric SPR absorption peak in particles with thicker shells. ${ }^{17}$

Experimentally, the absorption spectra of colloids of inert core gold shell particles are more convoluted due to the presence of other particles in the colloid, and absorbance due to the core material. Most gold shell particle colloids contain spherical gold nanoparticles and core particles without shells in addition to the desired core-shell particles. These other particles can contribute to the absorption characteristics of the colloid, particularly the gold nanoparticles. It could be argued that the SPR characteristics observed at higher wavelengths may actually be due to aggregates of spherical gold nanoparticles in the colloid rather than the core-shell particles. ${ }^{19-22}$ However, Averitt et al. have provided evidence suggesting the absorbance is indeed due to the core-shell particles by showing that experimentally obtained absorption spectra of $\mathrm{AuS}_{2}$-Au core-shell particles agree with those predicted by Mie theory, as shown in Figure 1.11. ${ }^{23}$ 

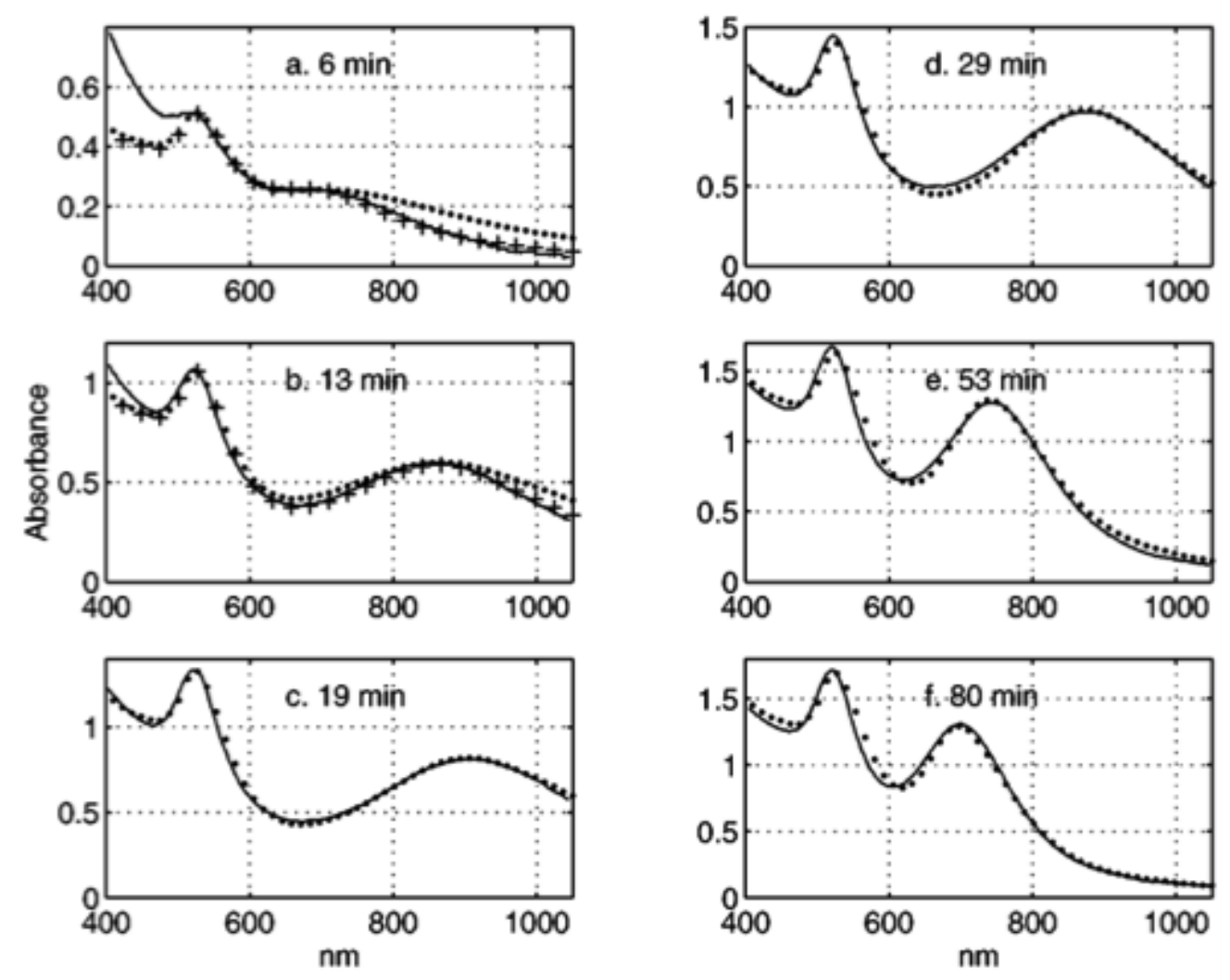

Figure 1.11: Absorbance spectra of $A u S_{2}$-Au core-shell particles predicted by Mie theory (dotted line) and gathered experimentally (solid line) by Averitt et al. after a. 6 minutes, b. 13 minutes, c. 19 minutes, d. 29 minutes, e. 53 minutes, $f .80$ minutes of growth. ${ }^{23}$

Spherical SPR active metal particles with non-metal shells have only one SPR absorption peak as the SPR active material is only the spherical particle, and the wavelength of this absorption peak is determined by the diameter of this sphere. However, the intensity of the SPR absorption peak is dependent on the shell thickness, as this affects the interaction of the metal with the electromagnetic radiation, and so with increased shell thickness the intensity of the SPR absorption peak is decreased. ${ }^{24}$ 


\subsection{Core-shell Particles}

Core-shell particles are being increasingly investigated as they allow for the tuning of physical and chemical properties of a material simply by using one particular material in conjunction with another. Coating a core material with a different material can allow the manipulation of optical, electrical and magnetic properties of the core material. ${ }^{25-27}$ Depositing a layer of shell material on a different material can allow for the coating material to be used with high surface area but less volume. For example, coating a cheap material with a catalytic material allows for less volume of the catalytic material to be used while still achieving the same catalytic activity, as the reactive surface area remains the same. ${ }^{28}$

\subsubsection{Gold Shells}

Gold nanoparticles are commonly used for their optical properties but also possess the useful property of being biologically inert, which allows their use in medical applications such as biological sensors for clinical diagnosis and in drug delivery systems. ${ }^{24,29}$ The optical properties of gold nanoparticles have been investigated for use in microscopy, however the SPR absorption wavelength is not optimal for near-infrared medical imaging. This problem could be solved by using core-shell particles with nanogold shells to increase SPR absorption wavelengths to the near-infrared region. However, synthesis of particles with even and continuous gold shells has proved challenging in the past.

Full gold shells have been successfully synthesised using methods that involved the deposition of ex-situ synthesised gold nanoparticles onto silica spheres capped with polyethyleneimine (PEI). However, as shown in Figure 1.12, these gold shells were very rough and uneven and also had large protruding clusters of gold nanoparticle aggregates. ${ }^{30}$ Syntheses using the same method but a different capping agent, 3-aminopropyltrimethoxysilane (APTMS), gave much different results. The use of spheres capped with APTMS did not lead to the formation of full gold shells, rather large clusters of gold nanoparticle aggregates formed that appeared to be only weakly associated with the silica surface. ${ }^{30}$ The failure to achieve full gold shells using APTMS as a capping agent where use of PEI succeeded can be attributed to the higher concentration of nitrogen in PEI than in APTMS. The higher concentration of 
nitrogen means that the silica spheres capped with PEI have greater affinity for the gold nanoparticles and so the interaction is stronger allowing the deposition of gold nanoparticles across the whole surface, forming full shells. UV-Vis absorption spectroscopy showed both samples presented in Figure 1.12 exhibited one broad peak absorbing from 500 to $700 \mathrm{~nm}$.
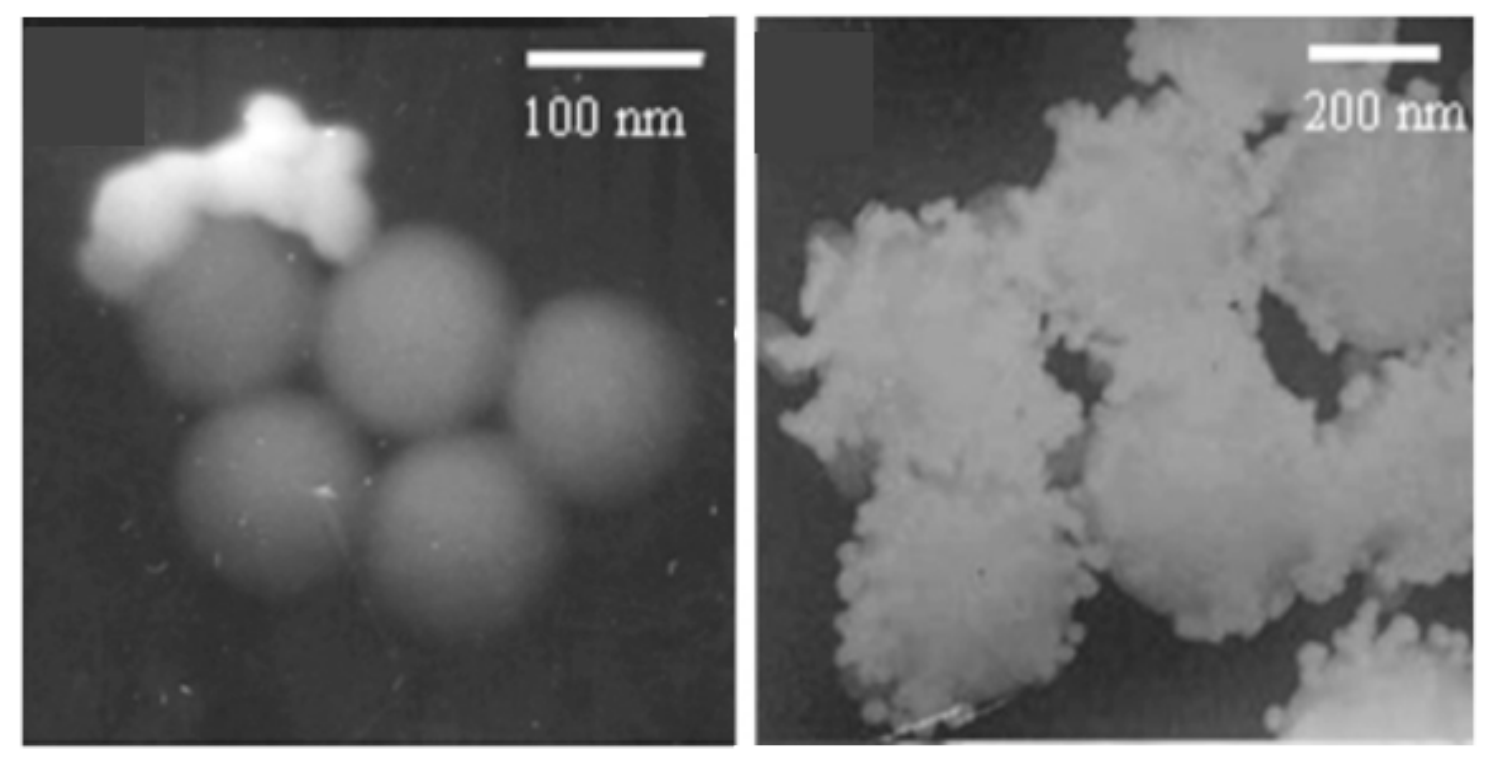

Figure 1.12: TEM images of gold nanoparticles (white areas) deposited onto silica core particles (grey spheres) capped with APTMS forming large clusters with little interaction (Left) or capped with PEI forming very rough shells with large protrusions (Right). ${ }^{30}$

Experiments involving the in-situ growth of gold nanoparticles onto prepared silica spheres simply by mixing silica with a solution of hydrogen tetrachloroaurate $\left(\mathrm{AuCl}_{4}{ }^{-}\right.$ions under acidic conditions), resulted in rough, uneven areas of gold nanoparticle aggregates on the silica surface, as shown in Figure 1.13. ${ }^{31}$ Further experiments included the use of 3-aminopropyltriethoxysilane (APTES) as a capping agent followed by in-situ growth of gold seed nanoparticles before in-situ growth of gold nanoshells. These syntheses resulted in some silica spheres being successfully surrounded by full gold shells. ${ }^{29}$ However, these gold shells were again found to be very rough and uneven and UV-Vis absorption spectroscopy showed one very broad 
SPR absorption peak, absorbing in the region of 500-900 nm, also shown in Figure $1.13 .^{29}$ The peaks in these UV-Vis absorption spectra are seen to shift to longer wavelength with increased shell thickness. As outlined in Section 1.2.3 SPR of Coreshell Particles, theory states that a thicker shell leads to a shorter wavelength shift; but this is not what is observed to happen in this case. This is due to the fact that the particles do not possess full gold shells, but rather many discrete gold nanoparticles. Hence, the UV-Vis absorption spectra show a red shift with greater shell thickness that is representative of the red shift associated with increasingly larger agglomerates of gold nanoparticles. The trend is seen to change between the thickest shells; in this case the UV-Vis absorption peak of the thicker shell is indeed blue shifted. This is due to the fact that the shell is now continuous and hence the SPR behaviour is due to a gold shell, not spherical gold nanoparticles. 

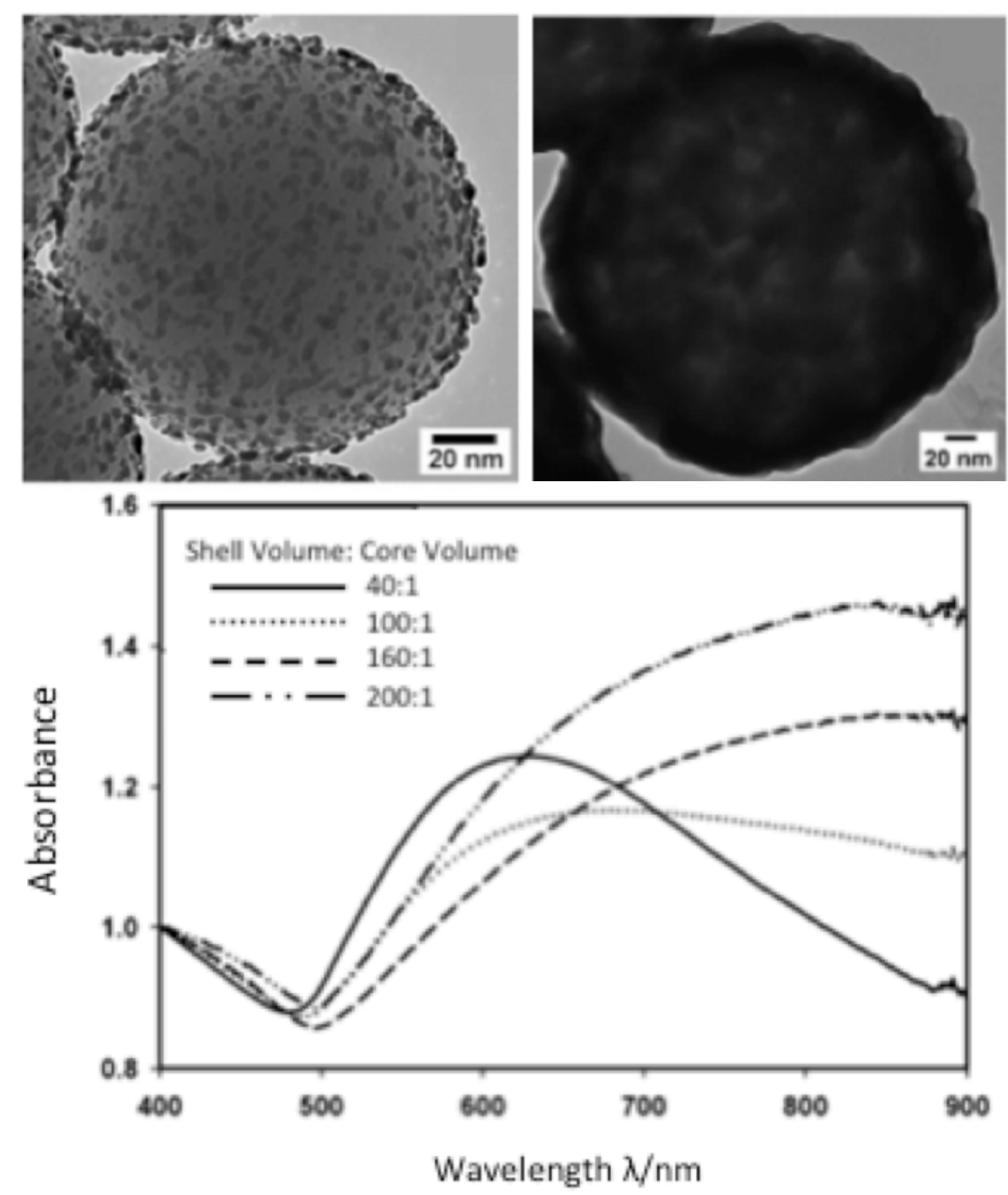

Figure 1.13: Top Left: TEM image of uncapped silica spheres with gold nanoparticle aggregates, ${ }^{31}$ Top Right: TEM image of a uneven full gold shell on APTES capped silica sphere, Bottom: UV-Vis absorption spectra of these full gold shell particles with varying shell to core volume ratios. ${ }^{29}$

In-situ growth of nanoshells onto silica surfaces decorated with gold seed nanoparticles is commonly achieved using a so-called "K-gold solution". 29,32,33 An aqueous solution of "K-gold" is prepared by adding $\mathrm{K}_{2} \mathrm{CO}_{3}$ to $\mathrm{HAuCl}_{4}$. The role of the $\mathrm{K}_{2} \mathrm{CO}_{3}$ in this solution is unclear, but the solution has been proven effective in producing gold shells. This solution must be kept in the dark and used within a few days. The in-situ growth of nanoshells is achieved by adding gold-seeded silica particles to the K-gold solution along with an external reducing agent, such as 
formaldehyde $^{33}$ or sodium borohydride. ${ }^{29,32}$ This shell growth method is usually preceded by ex-situ growth of gold nanoparticles, which are deposited onto the surface to serve as seed particles. ${ }^{32,33}$ The use of ex-situ synthesised gold nanoparticles as seeds allows for thinner shells to be achieved and was also found to lead to smoother shells than methods involving in-situ gold seeding that have been explored, as shown in Figure 1.14. The use of K-gold solution to prepare gold nanoshells is common, but the solution is light sensitive and degrades in some way. ${ }^{33}$ The exact chemical process of this degradation is unknown. The use of this reagent adds a difficulty to the synthesis that prevents the synthesis from being particularly compatible in commercial processes, meaning the development of a synthetic method without the use of this reagent would be desirable.

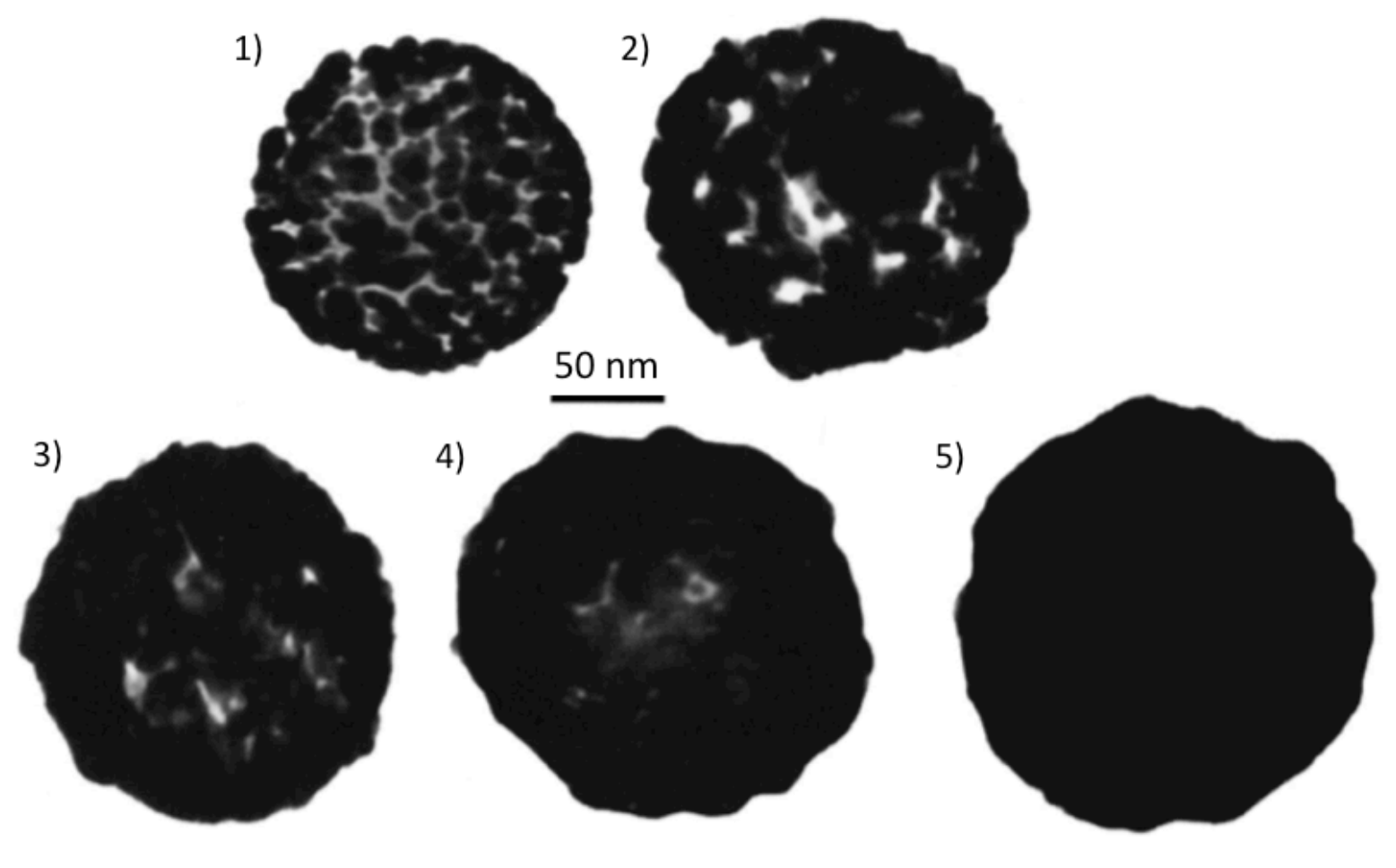

Figure 1.14: TEM images showing growth of gold nanoshells using K-gold solution after deposition of ex-situ synthesised gold nanoparticle seeds. ${ }^{32}$

Synthesis of gold shells has also been achieved by the deposition of ex-situ formed gold nanoparticles onto the surface of carboxylate-terminated polystyrene spheres, capped with 2-aminoethanethiol (AET). ${ }^{34}$ The shells were smoother and 
more even than those synthesised on silica spheres, as shown in Figure 1.15. However they were still reasonably rough allowing for different size dimensions to exist within each shell, which can influence the SPR of the particles. Also shown in Figure 1.15, the UV-Vis absorption spectroscopy showed that core-shell particles synthesised in this way again had one broad SPR absorption peak, absorbing in the range of approximately 500-700 $\mathrm{nm}$. The UV-Vis absorption spectra presented do not directly correlate to the TEM images shown. The red shift of these peaks is due to the increased aggregation of gold nanoparticles on the surface of the spheres in the shell growth process, rather than the increased thickness of a full shell. Larger diameter spherical particles have red shifted peaks; this agrees with the shift of the peaks in the spectra shown in Figure 1.15, and with the corresponding TEM images of similar particles with increased coverage of gold nanoparticles behaving as larger diameter gold particles.
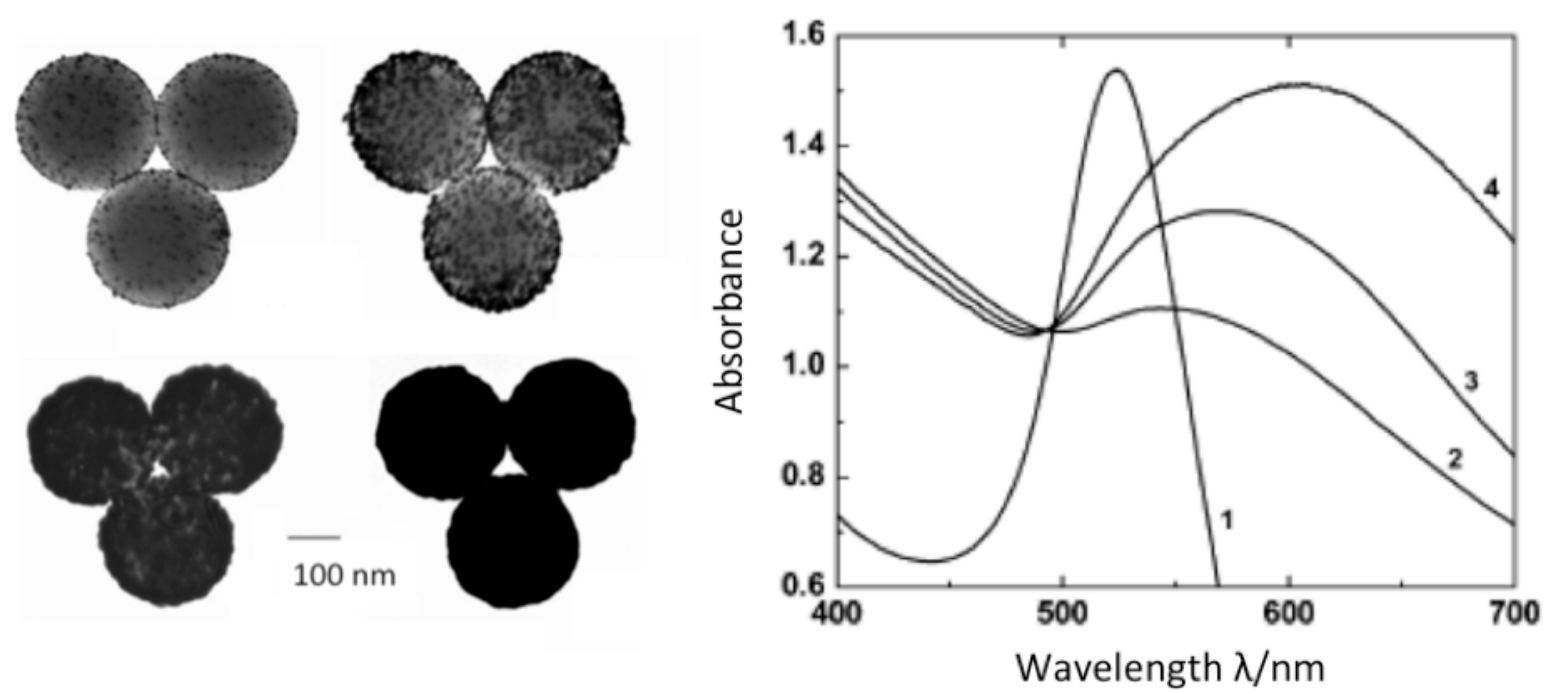

Figure 1.15: Left: TEM images showing the formation of a gold shell on a polystyrene sphere capped with AET, Right: UV-Vis absorption spectra of 1) Gold nanoparticles, and gold decorated polystyrene spheres with gold to polystyrene ratios of 2) 2.5:1, 3) $5: 1,4) 10: 1 .^{34}$

Previous synthetic methods have produced gold nanoshells with colours of purple, blue, green and yellow, with most involving the use of light sensitive K-gold 
solutions in addition to other reducing agents, do not achieve smooth regular shells and exhibit a broad UV-Vis absorption from 600 to $800 \mathrm{~nm}$.

The main objective of this research is to devise a potentially commercially viable method of synthesising smooth gold nanoshells on silica spheres in-situ with sharper UV-Vis absorption peaks between 500-800 $\mathrm{nm}$.

\subsubsection{Aggregation of Colloids}

A significant issue with stability of nanoparticle colloids is aggregation. The high surface area of nanoparticles causes a great energy cost to the system so aggregation is energetically favourable. Gold nanoparticle colloids are chemically inert so their stability is almost entirely dependent on their eventual aggregation due to electrostatic effects. Aggregation of gold nanoparticles causes changes in the SPR of the material. When two or more nanoparticles come together, within approximately half a particle diameter of each other, the surface plasmons are able to propagate between the particles and their SPR behaviour presents as that of one larger particle. ${ }^{35}$ As the SPR of a particle is dependent on it's size and shape, this aggregation therefore increases the effective particle size and changes the shape, thus changing the absorption characteristics of the sample. Aggregation of gold nanoparticles tends to cause the sample to appear a greyish colour as many different wavelengths are absorbed due to the sample containing particles of many different shapes and sizes, as opposed to absorbing at specific wavelengths due to the particular uniform size and shape of the nanoparticles. Aggregation can also cause unwanted effects in nanoparticle colloids of other materials. For example, aggregation of silica nanoparticle colloids leads to the monodispersity of the system being significantly decreased.

Several ways of preventing unwanted aggregation of nanoparticle colloids exist. The effects of aggregation can be prevented by utilising core-shell particles. Deposition of a secondary material around a nanoparticle core prevents the nanoparticles from coming within a specific distance of each other, that distance being twice the thickness of the deposited shell, even if nanoparticle aggregation occurs. 
One of the most widely used methods of preventing unwanted aggregation of nanoparticle colloids is steric stabilisation. Increased steric stability can be induced by the addition of a layer of organic functionality, such as a surfactant or capping agent, around the nanoparticles of a colloid. ${ }^{36}$ A layer of surfactant adsorbed onto the nanoparticles increases the steric stabilisation of the colloid by increasing the energy needed for the colloid to aggregate. This increased energy cost is due to the necessary displacement of the solvation spheres surrounding the surfactant layers to facilitate aggregation of the nanoparticles. The displacement of the solvation spheres is energetically unfavourable as it eliminates favourable interactions between the surfactant and the solvent and also decreases the entropy of the system; this is demonstrated in Figure 1.16.

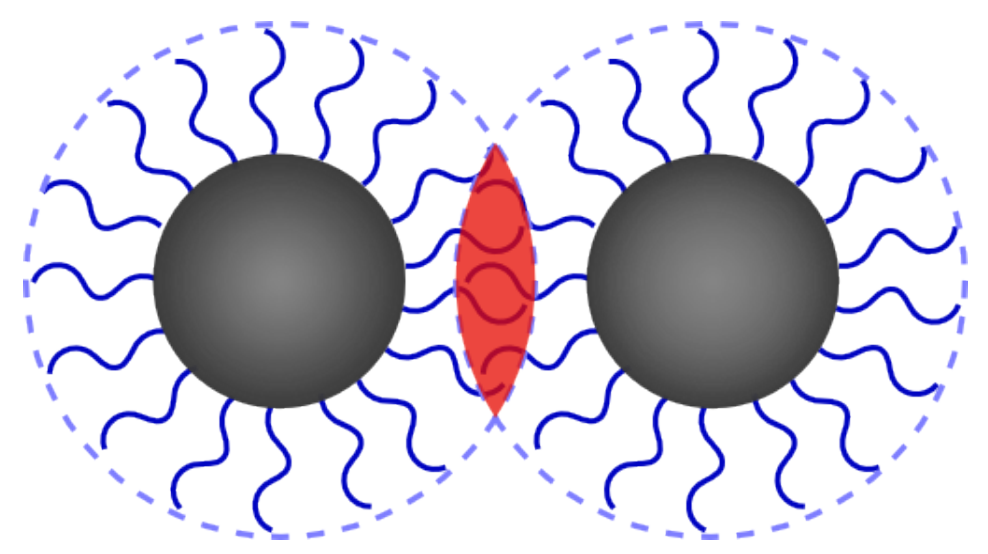

Figure 1.16: Schematic drawing demonstrating the factors involved in steric stabilisation of nanoparticles (grey) in a colloid due to the unfavourable interactions (red) between adsorbed surfactant molecules (blue). ${ }^{37}$

\subsubsection{Capping Agents}

Stabilisation of nanoparticle colloids using steric stabilisation also allows the functionalisation of the surface of the nanoparticles. The surfactant, or capping agent, used to stabilise the colloid can also be used to impart different reactivity to the surface. This emphasises the importance of making use of the right capping agent. For the synthesis of gold shells, it is important to have a capping agent that will make the 
surface of the cores possess a high affinity for gold. Functional groups containing nitrogen and sulfur have been shown to have great affinity for gold, ${ }^{38}$ hence the use of an amine or thiol terminated capping agent will more likely lead to successful gold shell synthesis. The capping agent must also bind to the surface of the core particles strongly and easily, so that the surface is evenly covered with this functionality, allowing for the synthesis of even shells. The use of silica as a core material allows for the use of silane terminated capping agents, as such silanes will bond strongly to the silica spheres.

In this research it is proposed that the capping agent will initiate reduction of the $\mathrm{AuCl}_{4}^{-}$adsorbed to the surface to create seed particles for the growth of gold shells. Hence, it is also important to consider the reducing power with respect to $\mathrm{AuCl}_{4}{ }^{-}$when selecting an appropriate capping agent.

\subsection{Silica}

Amorphous silica $\left(\mathrm{SiO}_{2}\right)$ is regarded as a chemically stable compound. In nature, amorphous silica can be found as the gemstone opal. Opal is made up of amorphous silica spheres with a regular close-packed arrangement. This regular closepacked arrangement is also seen in chemically synthesised silica spheres. Silica spheres are easily dispersed in aqueous solvent and can be readily functionalised using silane compounds. This allows for silica spheres to be used as a stable substrate in many applications and they are widely used as a stationary phase in column chromatography. 


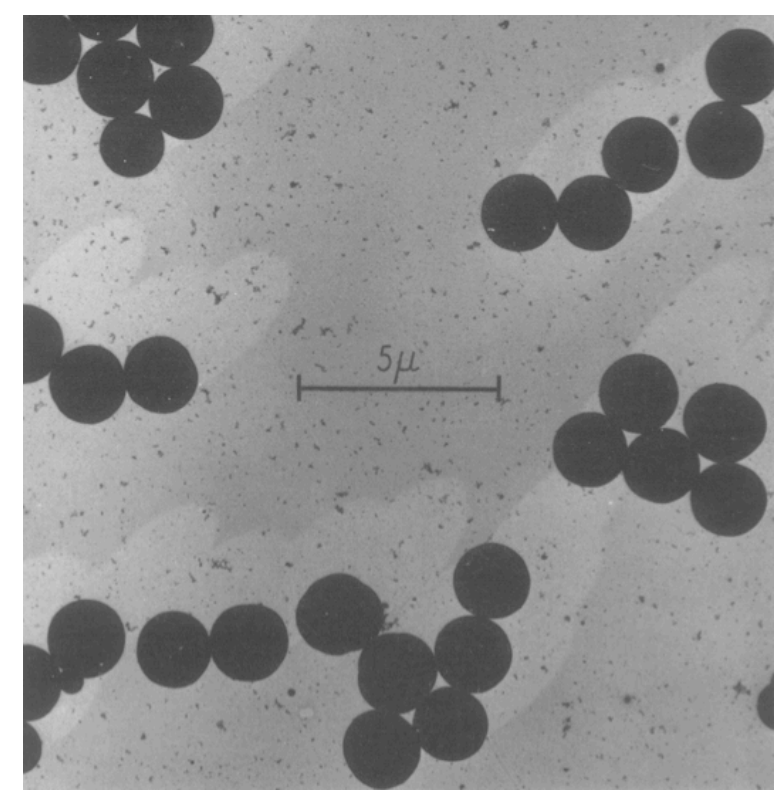

Figure 1.17: Electron micrograph of silica spheres synthesised via the Stöber method. $^{39}$

Silica spheres have previously been used as cores in silica-gold core-shell particles (see Section 1.3.1 Gold Shells). They provide a stable substrate that is easily functionalised. Another benefit of using silica as a core material is that the synthesis of silica spheres via the Stöber method allows for control over the final particle size. Due to these factors, silica spheres will be used as the core material in the core-shell particle syntheses investigated in this research.

\subsubsection{Stöber Process}

In 1968 Werner Stöber and colleagues published a method of preparing monodisperse samples of silica spheres by hydrolysing an alkyl orthosilicate starting material, now known as the Stöber Process. ${ }^{39}$ This method is widely used as it simply involves hydrolysis of either tetramethyl- or tetraethyl-orthosilicate (TMOS or TEOS, respectively) in ethanol using ammonia as a base. The hydrolysis proceeds by the reactions set out in Equations 2 and 3 below. 


$$
\begin{gathered}
\mathrm{Si}(\mathrm{OEt})_{4}+\mathrm{H}_{2} \mathrm{O} \rightarrow(\mathrm{OEt})_{3} \mathrm{Si}(\mathrm{OH})+\mathrm{EtOH} \\
(\mathrm{OEt})_{3} \mathrm{Si}(\mathrm{OH})+\mathrm{H}_{2} \mathrm{O} \rightarrow \mathrm{SiO}_{2}+3 \mathrm{EtOH}
\end{gathered}
$$

Equations 2 and 3: Hydrolysis of TEOS.

This simple synthesis merely requires the mixing of TEOS or TMOS with water, ammonia and ethanol as solvent under vigorous stirring for a few hours. The size of the silica spheres produced is determined by the relative quantities of reagents used in the synthesis, as shown in Figure 1.18. Using this process, silica spheres of diameter $0.1-1 \mu \mathrm{m}$ can be produced. For this research, particles of approximately 150-200 nm $(0.15-0.20 \mu \mathrm{m})$ will be synthesised.

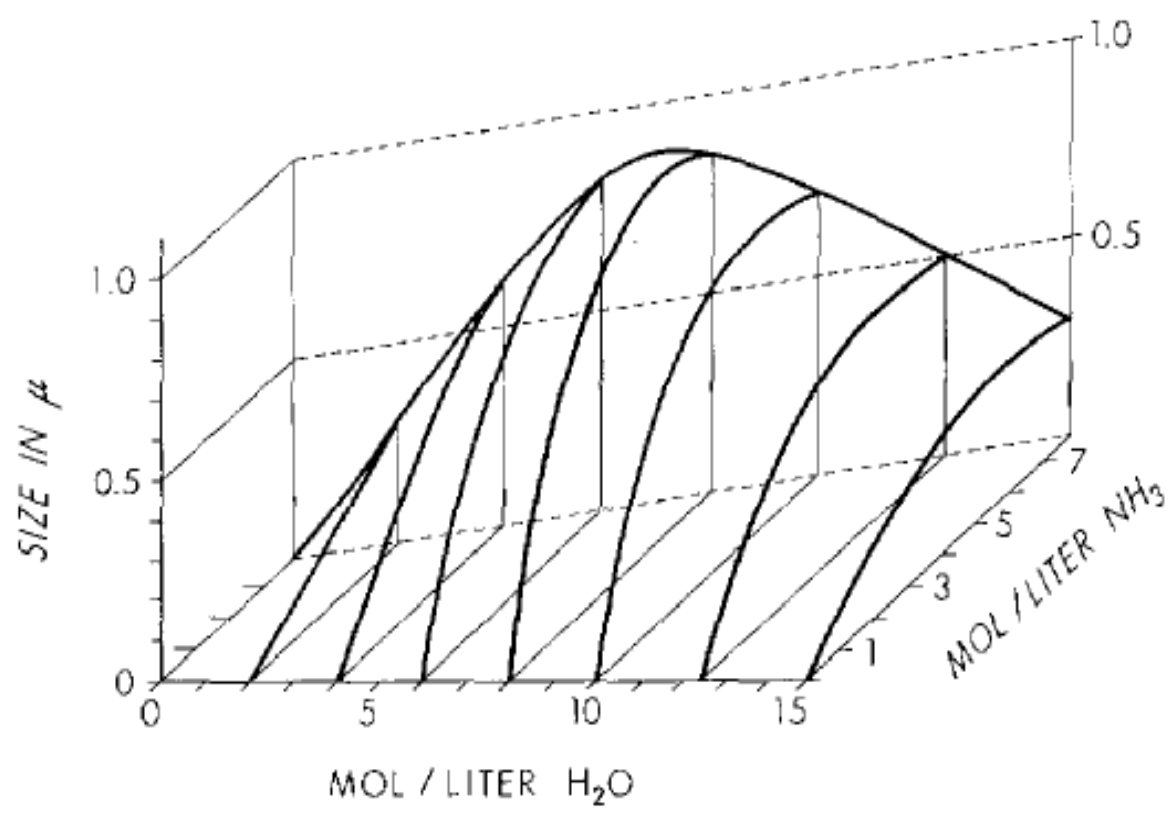

Figure 1.18: Graph showing dependence of particle size on reagent concentrations in Stöber process of synthesising silica spheres. ${ }^{39}$ 


\subsection{Titania}

Titania $\left(\mathrm{TiO}_{2}\right)$, like silica, is a network structure. However, titania exists in several forms with different crystal structures, most commonly rutile and anatase. ${ }^{40}$ As the physical properties of titania differ between structures, the different forms are used for different applications. Anatase has a higher band gap energy than rutile, so anatase is the preferred form for photovoltaic applications of the semiconductor material. ${ }^{41}$ However, it is becoming increasingly common to utilise a mixture of the two forms for enhanced photocatalytic activity. ${ }^{42}$ Rutile has a higher refractive index than anatase and hence rutile is used for whitening applications of titania. ${ }^{40}$

Titania is another potentially promising core material due to its low reactivity, semiconductor properties and high refractive index; it has been previously used in conjunction with gold to synthesise core-shell structures with interesting optical properties. ${ }^{43-45}$ Previously synthesised structures include functionalised titanium core particles decorated with gold nanoparticles synthesised by Pandikumar and co-workers. $^{43,44}$ This synthesis involved the use of the capping agent [3(2-aminoethylamino)propyl]trimethoxysilane to functionalise the preformed titania spheres and the use of sodium borohydride to reduce $\mathrm{AuCl}_{4}^{-}$to $\mathrm{Au}^{0}$. The resulting particles of this synthesis were found to be very irregular, as seen in Figure 1.19. The irregularity of the particles is determined to have arisen in the synthesis of the titania particles, as the UV-Vis spectra (also shown in Figure 1.19) exhibit sharp SPR absorption peaks, indicating reasonably monodisperse gold nanoparticles were formed. 

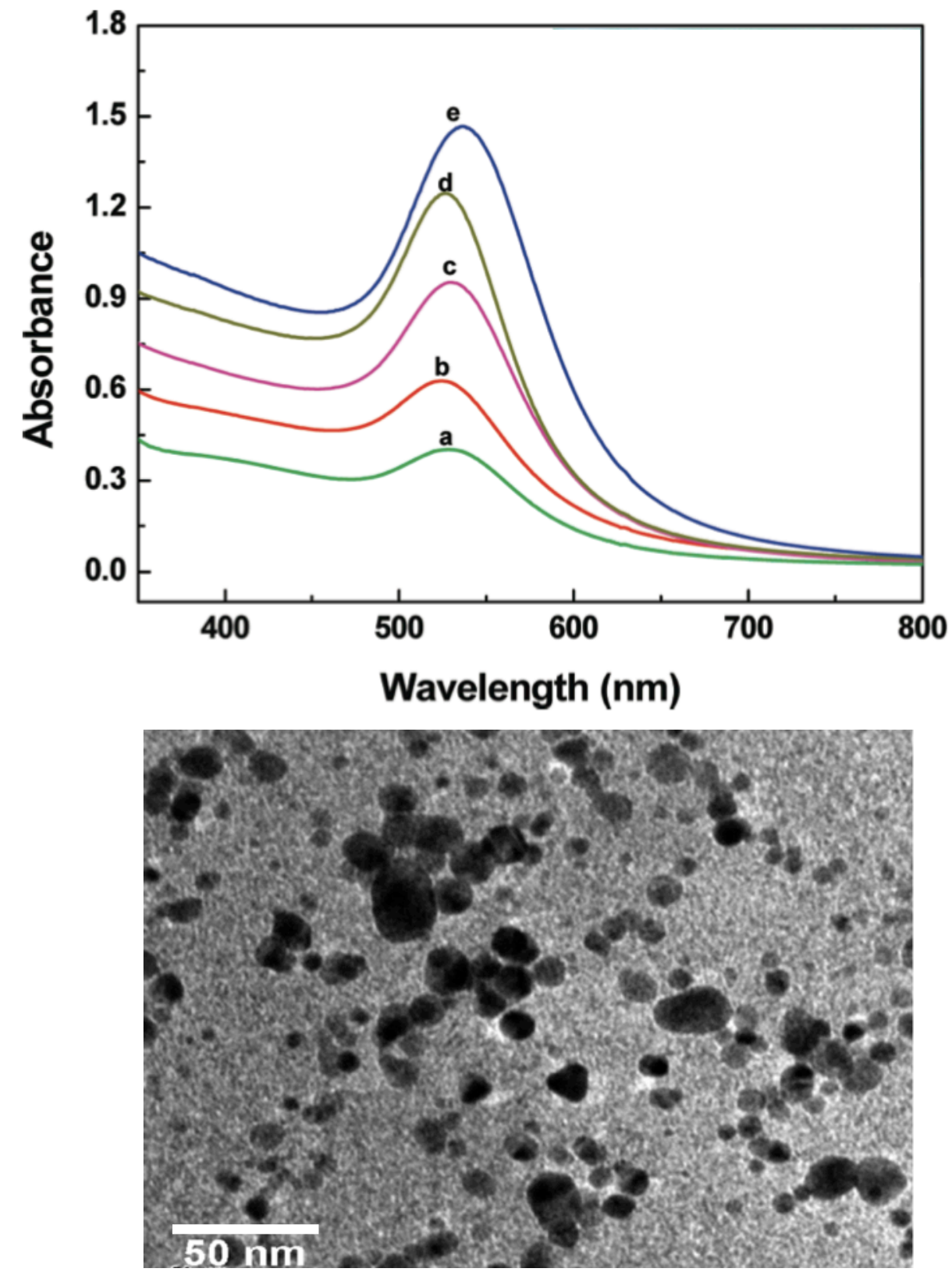

Figure 1.19: Top: UV-Vis of samples prepared by Pandikumar et al. with varying molar ratios of $\mathrm{TiO}_{2}$ :Au showing absorbance at approximately $520 \mathrm{~nm}$. $\mathrm{TiO}_{2}: \mathrm{Au}$ molar ratios are a) 100:1, b) 50:1, c) 33:1, d) 25:1 and e) 20:1. Bottom: TEM image of titania and gold particles synthesised by Pandikumar et al. ${ }^{43}$ 
As the refractive index of titania is much greater than that of silica, titania core gold shell particles should have significantly red shifted SPR absorption bands when compared to those of silica-gold particles. This shift in SPR absorption bands will lead to a different observed colour of the particles.

\subsection{Nanogold as a Colourant in Wool Fibres: An Application of SPR}

In 2008 Prof. James H. Johnston and Dr Kerstin Lucas (née Burridge) of Victoria University of Wellington developed a novel application of the SPR phenomenon of gold nanoparticles, by using coloured nanogold to colour New Zealand wool. ${ }^{38,46-50}$ This technology relies on the sulfur-containing amino acids found in wool (see Table 1.1) and the affinity of this sulfur for gold. The cross-linked thiols in some of these amino acids can also be used to reduce gold in-situ, reducing the number of other reagents used in the synthesis. ${ }^{46}$ The gold nanoparticles bind to these sulfur groups in the wool fibres and form a stable composite material with high value. In addition, gold nanoparticles formed ex-situ have the same affinity for sulfur and can be bound to wool fibres in the same way. 
Table 1.1: Sulfur containing amino acids in wool. ${ }^{51}$

Name of Amino Acid Side chain Concentration ( $\mu$ mol $g^{-1}$ )

\begin{tabular}{ccc} 
Cysteine & $-\mathrm{CH}_{2}-\mathrm{SH}$ & 10 \\
\hline Thiocysteine & $-\mathrm{CH}_{2}-\mathrm{S}-\mathrm{SH}$ & 5 \\
\hline Cysteic Acid & $-\mathrm{CH}_{2}-\mathrm{SO}_{3} \mathrm{H}$ & 10 \\
Cystine & $-\mathrm{CH}_{2}-\mathrm{S}-\mathrm{S}-\mathrm{CH}_{2}-$ & 460 \\
\hline Lanthionine & $-\mathrm{CH}_{2}-\mathrm{S}-\mathrm{CH}_{2}-$ & 5 \\
\hline Methionine & $-\left(\mathrm{CH}_{2}\right)_{2}-\mathrm{S}_{-}-\mathrm{CH}_{3}$ & 50
\end{tabular}

Since the colour of the composite gold-wool fibres depends entirely on the colour of the nanogold incorporated into the wool fibre matrix, different coloured gold-wool samples can be achieved by using gold nanoparticles of different sizes and shapes with different SPR absorbance properties. ${ }^{46}$ However, as the range of the achievable colour palette with spherical gold nanoparticles is currently somewhat limited to light pinks, purples and greys (see Figure 1.20), it is the aim of this research to extend the range to other hues, using gold core-shell particles.

To achieve this, a detailed study of the synthesis of core-shell particles will be required, along with an investigation of the parameters that control the resulting colour of the particles. Previous synthetic methods have shown that it is possible to synthesise silica-gold core-shell particles to obtain purple, blue, green or yellow colloids, depending on the thickness and completeness of the shell. ${ }^{32}$ As the gold component comprises the shell of these particles, their reactivity towards wool is similar to that of spherical gold particles. This allows for the coloured core-shell particles to be attached to wool fibres in a similar way as that developed by Johnston and Lucas. 


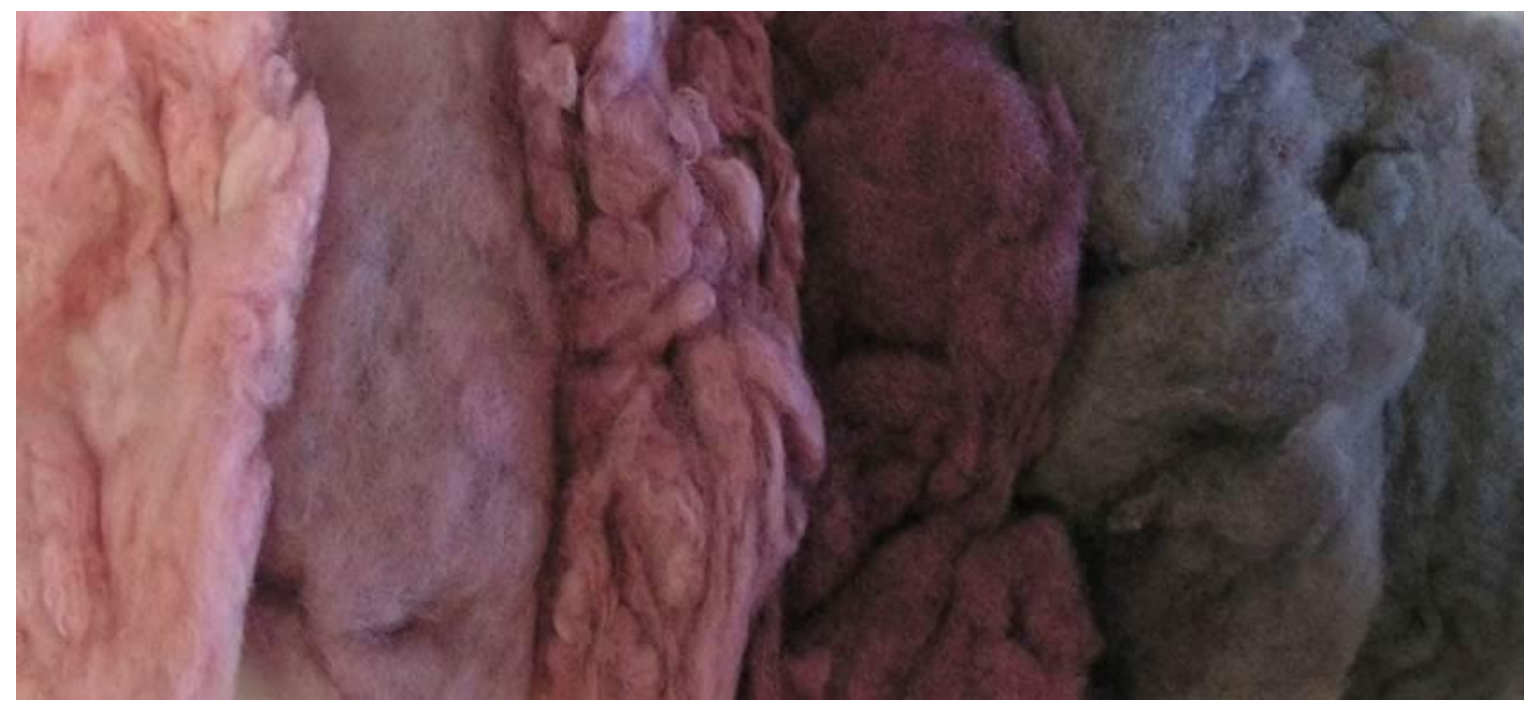

Figure 1.20: A selection of colours achieved using spherical gold nanoparticles as colourant in wool.

\subsection{Project Aims}

Gold wool is a promising commercial application of the SPR of gold nanoparticles but has a somewhat limited colour range available by using only spherical gold nanoparticles. This project aims to extend this colour range by synthesising and characterising silica core gold shell particles for the commercial colouring of wool. Silica will be used as the core material in this application as it is reasonably chemically inert and thus provides a stable substrate for the SPR active gold shells. Titania will also be investigated as a core material. As titania has a much greater dielectric constant than silica, the SPR characteristics of the surrounding gold shell will be shifted, providing a greater range of colours.

Currently published methods for synthesising these silica-gold core-shell particles present significant challenges in scale-up and commercialisation, such as multiple steps in the synthetic scheme and the use of light-sensitive reagents. This project aims to devise a simpler synthetic method involving minimal steps, mild temperatures and reagents that are light stable. The approach will utilise syntheses involving the in-situ growth of seed particles for the gold shells, decreasing the 
number of steps required in the synthesis. Using capping agents with the ability to reduce $\mathrm{AuCl}_{4}^{-}$to gold nanoparticles will facilitate the in-situ seeding of gold nanoparticles. This seeding step will be allowed to proceed above room temperature to make the progression of the reaction more favourable. The seeding step will be enhanced by the addition of sodium borohydride, a strong reductant that will reduce any $\mathrm{AuCl}_{4}{ }^{-}$not reduced by the capping agent. The growth of the gold shells will utilise hydroxylamine, a weak reducing agent capable of reducing $\mathrm{AuCl}_{4}{ }^{-}$onto surfaces already decorated with gold metal, so as to reduce gold onto the core surface and not into the solution. ${ }^{52}$ The use of a combination of reducing agents in conjunction with $\mathrm{AuCl}_{4}{ }^{-}$to grow the gold shells will eliminate the need for the lightsensitive K-gold solution.

After a thorough investigation of previous synthetic methods of gold shells, silica spheres have been chosen as an appropriate core material due to the ease of synthesis with the ability to control the final particle size via the Stöber method, and the ease of functionalisation with capping agents. In this research it is proposed that the capping agent will initiate reduction of the $\mathrm{AuCl}_{4}{ }^{-}$adsorbed to the surface to create seed particles for the growth of gold shells. Hence, it is also important to consider the reducing power with respect to $\mathrm{AuCl}_{4}{ }^{-}$when selecting an appropriate capping agent. As outlined above, it is desirable to have either amine or thiol functionality on the capping agent. It is also desirable to have silane functionality in the capping agent. (3-mercaptopropyl)-trimethoxysilane (MPTMS) has a terminal thiol group as well as a terminal silane. Another beneficial property of MPTMS is that thiol groups have been shown to reduce $\mathrm{AuCl}_{4}{ }^{-}$to gold nanoparticles. ${ }^{38,47}$ These factors should allow for successful synthesis of silica-gold core-shell particles and hence its use will be investigated. Some amines have been shown to reduce $\mathrm{AuCl}_{4}{ }^{-}$. However these are only polymeric amines such as polyethyleneimine (PEI). ${ }^{53} \mathrm{PEI}$ does not have a silane functionality allowing for strong bonding to the silica cores, but it has previously been proven to bind to silica spheres as a capping agent (see Section 1.3.1 Gold Shells), so the use of PEI as a capping agent will also be investigated.

The particles will be used to colour the wool by the same methods used for spherical gold nanoparticles. As the outside shell of the particles is gold, the 
interactions with the sulfur-containing amino acids in the wool fibres will remain, and may be utilised for the binding of the particles to the wool. 


\section{Experimental}

\subsection{Materials}

\subsubsection{Purchased Materials}

All water used in experiments was distilled water unless specified. All ethanol used in experiments was absolute ethanol unless specified. (3-aminopropyl)trimethoxysilane (APTMS) 97\%, (3-mercaptopropyl)-trimethoxysilane (MPTMS) $95 \%$, sodium borohydride powder $\geq 98 \%$, low molecular weight water-free polyethyleneimine (PEI), tetraethylorthosilicate (TEOS) $\geq 99 \%$, glycine $\geq 99 \%$, tannic acid (TA) ACS reagent $\geq 99 \%$, and sodium citrate tribasic dihydrate (trisodium citrate, TSC) ACS reagent $\geq 99 \%$ were purchased from Sigma-Aldrich. Hydroxylammonium chloride $\geq 99 \%$ was purchased from AnalaR. Hydrochloric acid 37\% was purchased from Panreac. Ammonia solution 35\% AR was purchased from Pure Science. Aeroxide $^{\circledR} \mathrm{TiO}_{2}$ P25 (P1S1264) was purchased from Degussa AG, containing approximately $70 \%$ anatase and $30 \%$ rutile. Gold metal was sourced from NZ gold bullion supplies. Approximately $20 \mu \mathrm{m}$ fibre diameter, scoured merino top wool was supplied from Ashford Handicrafts Ltd., Timaru.

\subsubsection{Solutions}

The following solutions were prepared for use in experiments and will be referred to as standard. A solution of $5 \mathrm{wt} \%$ TSC was prepared by adding $0.5 \mathrm{~g}$ of TSC to a volumetric flask and making up to $10 \mathrm{~mL}$ with water. A solution of $1 \mathrm{wt} \%$ TA was prepared by adding $0.5 \mathrm{~g}$ of TA to a volumetric flask and making up to $50 \mathrm{~mL}$ with water. A solution of $5 \mathrm{M} \mathrm{HCl}$ was prepared by adding $4.17 \mathrm{~mL}$ of concentrated $\mathrm{HCl}$ to $5.83 \mathrm{~mL}$ of water. A solution of approximately $0.19 \mathrm{M} \mathrm{NH}_{2} \mathrm{OH}$ was prepared by adding $0.265 \mathrm{~g}$ of hydroxylammonium chloride to a volumetric flask and making up to $20 \mathrm{~mL}$ with water. Solutions of $\mathrm{NaBH}_{4}$ were prepared by adding $0.028 \mathrm{~g}$ of sodium borohydride powder to a volumetric flask and making up to $100 \mathrm{~mL}$ with either water or ethanol depending on the solvent of the sample to which 
the $\mathrm{NaBH}_{4}$ was to be added. $\mathrm{NaBH}_{4}$ solutions were prepared when needed and used immediately.

A solution of $\mathrm{AuCl}_{4}{ }^{-}$in acidic conditions was prepared by dissolving metallic gold in aqua regia. Aqua regia is a 3:1 mixture of concentrated hydrochloric and nitric acids. This process is followed by the addition of urea to the solution; this converts the excess nitric acid into nitrogen and carbon dioxide gases and water. This solution was decanted to remove excess urea and distilled down to approximately $30 \mathrm{~mL}$ to remove excess hydrochloric acid. This concentrated solution was then made up to $250 \mathrm{~mL}$ with distilled water. The concentration of this solution was measured with atomic absorption spectroscopy and found to be $3.08 \mathrm{wt} \%$.

\subsection{Capped Silica Spheres}

\subsubsection{Silica Spheres}

Silica was synthesised using modified Stöber $\operatorname{process}^{39}$ (see Section 1.4.1 Stöber Process) as used by Xue et al. ${ }^{30} 1.0 \mathrm{~mL}$ of distilled water and $1.5 \mathrm{~mL}$ of TEOS were added to $50 \mathrm{~mL}$ of ethanol and heated to $40{ }^{\circ} \mathrm{C}$ under continuous stirring. When the solution reached $40{ }^{\circ} \mathrm{C}, 4.2 \mathrm{~mL}$ of $\mathrm{NH}_{3}$ was added and the solution was stirred and kept at $40{ }^{\circ} \mathrm{C}$ for 3 hours. Another $1 \mathrm{~mL}$ of TEOS was added and stirring and heating continued for another 3 hours. This reaction mixture was added to approximately equal volume of drum acetone and shaken vigorously to break the microemulsion state before being centrifuged at approximately $2500 \mathrm{rpm}$ for 30 minutes. The sample was then washed by being decanted, redispersed in ethanol via sonication and centrifuged for another 30 minutes at approximately $2500 \mathrm{rpm}$. This washing procedure was carried out 3 times followed by a fourth washing with distilled water in the place of ethanol, before the resulting solid was redispersed in distilled water.

\subsubsection{PEI Capped Silica Spheres}

Solutions of $1 \mathrm{wt} \%, 5 \mathrm{wt} \%$ and $10 \mathrm{wt} \%$ PEI in water were prepared and the $\mathrm{pH}$ adjusted to approximately 8 using hydrochloric acid. $10 \mathrm{~mL}$ of $1 \mathrm{wt} \%$ silica colloid was added to $10 \mathrm{~mL}$ of each PEI solution, and shaken overnight. The reaction mixtures were then centrifuged at approximately $2500 \mathrm{rpm}$ for 30 minutes. The 
samples were then washed by being decanted, redispersed in distilled water via sonication and centrifuged for another 30 minutes at approximately $2500 \mathrm{rpm}$. This washing procedure was carried out 3 times before the resulting solids were redispersed in distilled water to give approximately $1 \mathrm{wt} \%$ colloids.

A colloid was also prepared containing $2 \mathrm{~mL} 10 \mathrm{wt} \%$ PEI and $2 \mathrm{~mL} 1 \mathrm{wt} \%$ silica in ethanol, this colloid was left at $50{ }^{\circ} \mathrm{C}$ for 5 days. The reaction mixture was then centrifuged and washed as above and redispersed in ethanol. This colloid is subsequently referred to as sample SP.

\subsubsection{MPTMS Capped Silica Spheres}

The method used for capping silica with MPTMS was based on that used by Meeks et al. ${ }^{54}$ Approximately $0.5 \mathrm{~g}$ of silica (dispersed in distilled water) was added to $1.56 \mathrm{~mL}$ of MPTMS and left in a shaking water bath at $50{ }^{\circ} \mathrm{C}$ for 7 days. The reaction mixture was centrifuged at approximately $2500 \mathrm{rpm}$ for 30 minutes. The sample was then decanted, redispersed in ethanol and centrifuged for another 30 minutes at approximately $2500 \mathrm{rpm}$ to wash the solid. The solid was washed a total of three times in this way, before being redispersed in ethanol.

\subsection{Capped Titania Spheres}

\subsubsection{Titania Spheres Capped with MPTMS}

Titania spheres were capped with MPTMS using a similar method to that used to cap silica, however more MPTMS was used, as there was more surface area in the titania samples with smaller diameter particles. Approximately $0.01 \mathrm{~g}$ of titania was dispersed in $50 \mathrm{~mL}$ of distilled water, to this solution, $1.56 \mathrm{~mL}$ of MPTMS was added and the final colloid was left in a shaking water bath at $50{ }^{\circ} \mathrm{C}$ for 7 days.

\subsection{Gold Nanoshells on Capped Silica Spheres}

\subsubsection{Ex-situ Seeding on PEI Capped Silica Spheres}

Two gold nanoparticle colloids were prepared as ex-situ seed particles. Colloid A was prepared according to Xue et al. ${ }^{30}$ by adding $0.2558 \mathrm{~mL}$ of $\mathrm{AuCl}_{4}{ }^{-}$ 
solution and $2 \mathrm{~mL}$ of TSC solution to $100 \mathrm{~mL}$ of water at $95{ }^{\circ} \mathrm{C}$. This solution was left boiling and stirring for 10 minutes, removed from the heat and stirred for a further 15 minutes. A further $0.30 \mathrm{~mL}$ of TSC solution was then added and stirred for a further 30 minutes. Colloid B was prepared by adding $38.5 \mu \mathrm{L}$ of $\mathrm{AuCl}_{4}{ }^{-}$solution and $125 \mu \mathrm{L}$ of TSC solution to $100 \mathrm{~mL}$ of distilled water at $95^{\circ} \mathrm{C}$. This solution was stirred and heated for 2 hours.

Samples containing $1 \mathrm{~mL}$ of PEI capped silica spheres and $2 \mathrm{~mL}, 1 \mathrm{~mL}$ or $0.5 \mathrm{~mL}$ of a gold colloid (either colloid A or colloid B, as prepared above) were prepared and made up to $5 \mathrm{~mL}$ with distilled water. The samples were placed on a shaker table for 24 hours.

\subsubsection{In-situ Seeding on PEI Capped Silica Spheres}

Samples containing $1 \mathrm{~mL}$ of a $1 \mathrm{wt} \%$ solution of PEI capped silica and $1 \mathrm{~mL}$ or $2 \mathrm{~mL}$ of a $100 \mathrm{ppm} \mathrm{AuCl}_{4}{ }^{-}$solution, with or without additional $2 \mathrm{~mL}$ of $1 \mathrm{wt} \%$ PEI solution, were placed in a shaking water bath at $50{ }^{\circ} \mathrm{C}$ for 7 days.

\subsubsection{In-situ Seeding on PEI Capped Silica Spheres with Hydroxylamine}

A sample was prepared containing $10 \mathrm{~mL}$ of $0.1 \mathrm{wt} \%$ PEI capped silica in ethanol and $50 \mu \mathrm{L}$ of $\mathrm{AuCl}_{4}{ }^{-}$. This sample was left at $50{ }^{\circ} \mathrm{C}$ for 5 days, before $0.209 \mathrm{~mL}$ of hydroxylamine solution was added to the sample. The sample was then kept at $50{ }^{\circ} \mathrm{C}$ for a further 3 days, with $50 \mu \mathrm{L}$ of $\mathrm{AuCl}_{4}{ }^{-}$added each day.

\subsubsection{In-situ Seeding on MPTMS Capped Silica Spheres}

Samples were prepared containing $1 \mathrm{~mL}$ of $1 \mathrm{wt} \%$ MPTMS capped silica, $\mathrm{AuCl}_{4}{ }^{-}$solution and $19 \mathrm{~mL}$ of distilled water. These samples were stirred and heated to either $75^{\circ} \mathrm{C}$ or $98^{\circ} \mathrm{C}$ for 2 hours. 


\subsubsection{In-situ Seeding on MPTMS Capped Silica Spheres with}

\section{Hydroxylamine}

The methods of gold nanoshell synthesis using hydroxylamine were based on variations of the method used by Brown and Natann ${ }^{52}$ to reduce gold.

A sample was prepared, containing $1 \mathrm{~mL}$ of $1.2 \mathrm{wt} \%$ MPTMS capped silica, $50 \mu \mathrm{L}$ of $\mathrm{AuCl}_{4}{ }^{-}$and $9 \mathrm{~mL}$ of ethanol, the sample was put in a shaking water bath at $50{ }^{\circ} \mathrm{C}$ for 24 hours. After 24 hours, $0.209 \mathrm{~mL}$ of $\mathrm{NH}_{2} \mathrm{OH}$ solution was added and the sample returned to the shaking water bath at $50{ }^{\circ} \mathrm{C}$ for more than 6 hours. Another $50 \mu \mathrm{L}$ of $\mathrm{AuCl}_{4}^{-}$was added and the sample returned to the shaking water bath for more than 6 hours. This addition was repeated so the total amount of $\mathrm{AuCl}_{4}{ }^{-}$in the sample was $150 \mu \mathrm{L}$, and the final sample was then left in the shaking water bath at $50{ }^{\circ} \mathrm{C}$ for 24 hours.

\subsubsection{In-situ Seeding on MPTMS Capped Silica Spheres with $\mathrm{NaBH}_{4}$ and Hydroxylamine}

Samples were prepared containing $1 \mathrm{~mL}$ of $1.2 \mathrm{wt} \%$ MPTMS capped silica spheres, $50 \mu \mathrm{L}$ of $\mathrm{AuCl}_{4}{ }^{-}$and $9 \mathrm{~mL}$ of ethanol and left at room temperature for 3 hours. $\mathrm{NaBH}_{4}$ solution was then added to each sample and left at room temperature overnight. Subsequently, $0.209 \mathrm{~mL}$ of $\mathrm{NH}_{2} \mathrm{OH}$ was then added to each sample and put in a shaking water bath at $50{ }^{\circ} \mathrm{C}$ for more than 6 hours. After this time, the samples were cooled and another $50 \mu \mathrm{L}$ of $\mathrm{AuCl}_{4}{ }^{-}$was added and the samples were returned to the shaking water bath at $50{ }^{\circ} \mathrm{C}$ for more than 6 hours, this addition was repeated so each of the samples contained a total of $150 \mu \mathrm{L}$ of $\mathrm{AuCl}_{4}{ }^{-}$.

\subsection{Colouring Wool with Gold Nanoparticles}

Before colouring, all wool samples were washed in hot tap water. Wool was added to hot tap water, and completely wet. This water was then changed and left to soak for 10 minutes, changed again and left for another 10 minutes. This preparation procedure is in accordance with previously developed methods. ${ }^{50}$ 


\subsubsection{Spherical Gold Nanoparticles}

These samples were prepared using a proprietary method. A gold colloid was prepared of which the $\mathrm{pH}$ was lowered. Wool was added to the $\mathrm{pH}$ adjusted gold colloid along with a wetting agent, and soaked overnight until all the gold colloid was absorbed. The coloured wool was then rinsed in tap water and left to dry at ambient temperature.

\subsubsection{Gold Core-shell Nanoparticles}

The $\mathrm{pH}$ of a colloid of washed core-shell nanoparticles dispersed in $20 \mathrm{~mL}$ of water was adjusted to approximately 1.4. A sample of $0.08 \mathrm{~g}$ of washed wool was added to this colloid. The wool was soaked at room temperature overnight. The coloured wool was then rinsed in tap water and left to dry on a windowsill.

\subsection{Characterisation Methods}

\subsubsection{UV-Vis Absorption Spectroscopy}

UV-Vis absorption spectroscopy was carried out on a Varian Cary 100 scan spectrometer UV-Vis spectrophotometer. Spectra were collected between $400-800 \mathrm{~nm}$. Samples were prepared by diluting or concentrating colloids to achieve appropriate absorbance levels.

\subsubsection{X-ray Diffraction}

X-ray diffraction (XRD) measurements were taken on a PANalytical X'Pert Pro MPD diffractometer with copper X-ray source, with a voltage of $45 \mathrm{kV}$ and a current of $40 \mathrm{~mA}$. Samples were prepared by dropcasting onto a Perspex blank disc. Scans were run from $10-80^{\circ}(2 \theta)$ at a scan rate of $1.32^{\circ}(2 \theta) \mathrm{min}^{-1}$.

\subsubsection{Scanning Electron Microscopy}

Scanning electron microscopy (SEM) was performed on two instruments. The first, a JEOL-6500F Field Emission Scanning Electron Microscope fitted with an Energy Dispersive X-ray Analysis Spectroscopy (EDS) probe for elemental mapping 
of sample composition. Colloid samples were prepared by dropcasting colloids onto silicon wafers mounted on aluminium stubs. These samples were coated with $8 \mathrm{~nm}$ of carbon. Coating with carbon is a common practice in SEM preparation as it provides a conductive surface to reduce charging while imaging. Wool samples were prepared by placing fibres onto carbon tape on aluminium stubs. These samples were also coated with $8 \mathrm{~nm}$ of carbon. Imaging was obtained in both secondary electron imaging (SEI) and backscatter (BS) modes with an accelerating voltage of $15 \mathrm{kV}$.

Secondly, a JEOL-JSM-6610LA was also used. Colloid samples were again prepared by dropcasting colloids onto silicon wafers mounted on aluminium stubs, however these samples were either not coated or coated with 4-16 nm of carbon or $10 \AA ̊$ of platinum as required. Wool samples were again prepared by placing fibres on carbon tape on aluminium stubs, and coated with $8 \mathrm{~nm}$ of carbon. Imaging was again obtained with an accelerating voltage of $15 \mathrm{kV}$.

Both instruments were also used to carry out Energy Dispersive X-ray Spectroscopy (EDS) to give details on the elemental composition of samples. EDS spectra and maps were carried out with an accelerating voltage of $15 \mathrm{kV}$.

\subsubsection{Transmission Electron Microscopy}

Transmission electron microscopy (TEM) was performed on a JEOL-2010 Transmission Electron Microscope. Samples were prepared by placing a drop of colloid onto a copper TEM grid and allowing the solvent to evaporate slowly. Images were obtained using an accelerating voltage of $200 \mathrm{kV}$.

Scanning Transmission Electron Microscopy (STEM) was also used to carry out EDS analysis, also on a JEOL-2010 TEM with an accelerating voltage of $200 \mathrm{kV}$ to give details on the elemental composition of samples.

\subsubsection{Size Measurements}

Size measurements using dynamic light scattering were carried out on a Malvern Instruments Nanoseries Zetasizer Nano-ZS. Samples were diluted to appropriate concentrations using the respective solvent, ethanol or water. The 
measurements were taken at $25^{\circ} \mathrm{C}$, each measurement consisted of three runs and each measurement was taken three times and averaged.

\subsubsection{Colour Measurements}

The colour of wool fibres was measured using a Hunterlab ColourQuest spectrophotometer. This instrument can be used to measure CIE Lab values of samples (shown in Figure 2.1), as well as measure the visible reflectance spectra.

The CIE Lab values are coordinates that represent a colour in the CIE Lab colour space. Three axes define this colour space. The $\mathrm{L}^{*}$ axis ranges from black $\left(\mathrm{L}^{*}=0\right)$ to white $\left(\mathrm{L}^{*}=100\right)$ and indicates lightness, which is determined by the cube root of the relative luminance. The $\mathrm{a}^{*}$ axis ranges from green $\left(-\mathrm{a}^{*}\right)$ to red $\left(+\mathrm{a}^{*}\right)$ and the $b^{*}$ axis ranges from blue $\left(-b^{*}\right)$ to yellow $\left(+b^{*}\right)$. The CIE Lab colour space is used as it includes every colour perceivable.

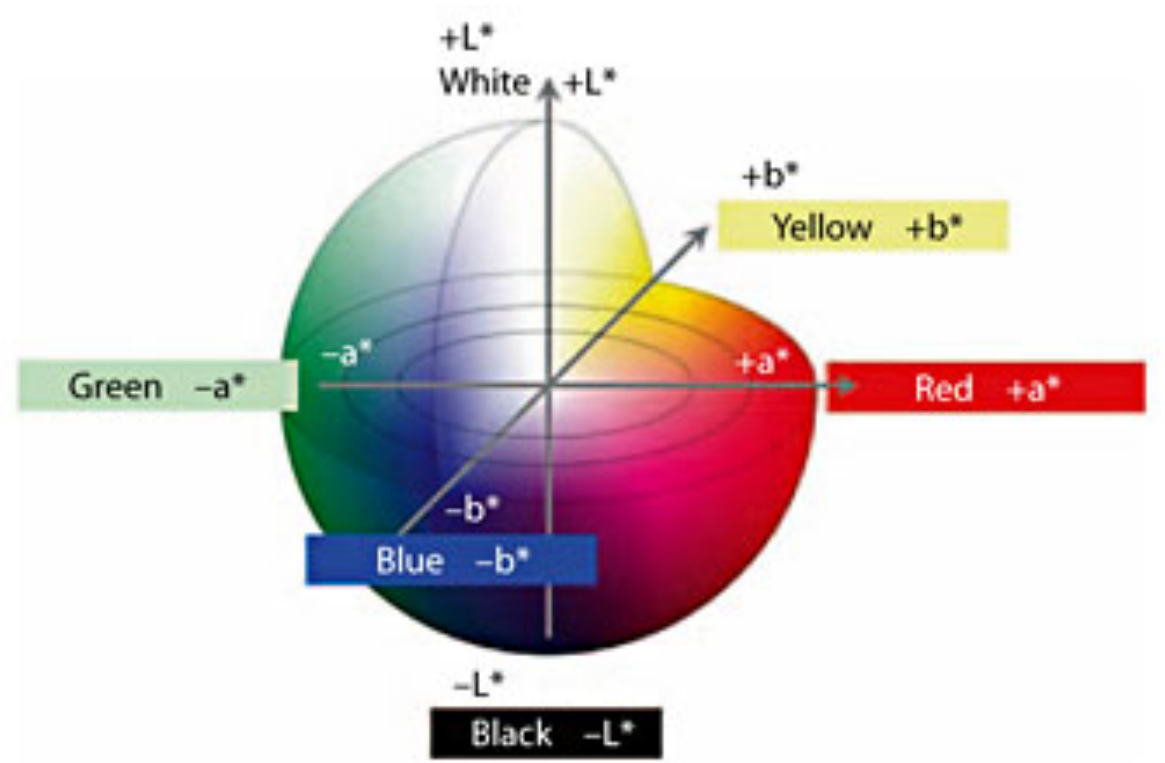

Figure 2.1: CIE Lab colour space. ${ }^{55}$

The reflectance spectra obtained from the ColourQuest instrument are represented in percentage reflectance. This percentage reflectance is divided by one 
hundred to give absolute reflectance. Using the absolute reflectance, a Kubelka-Munk transformation can be performed on the reflectance data to give spectra representing the colour yield of the sample. Although absorbance is not measured, these Kubelka-Munk transformed reflectance spectra can demonstrate the approximate SPR behaviour of the sample. The Kubelka-Munk transformation is represented in Equation 2.1, where $\mathrm{R}$ is the absolute reflectance, $\mathrm{K}$ is the absorption coefficient and $\mathrm{S}$ is the scattering coefficient.

$$
F(R)=\frac{(1-R)^{2}}{2 R}=K / S
$$

Equation 2.1: The Kubelka-Munk transformation. 


\section{Characterisation of Silica and Titania}

This chapter discusses the results of silica synthesis and capping procedures of PEI and MPTMS on silica and titania spheres.

\subsection{Synthesis of Silica Spheres}

Silica spheres were synthesised using a modified Stöber process as described by Xue et al..$^{30}$ (see Section 2.2.1 Silica Spheres). After addition of ammonia, a white precipitate was formed and the sample was very pale translucent white, similar in appearance to very dilute milk. After the additional TEOS was added to the sample, the amount of white precipitate present increased and the sample turned almost opaque white. This solid was washed and redispersed easily in water. The solid's white appearance and easy dispersion in water were properties characteristic of silica spheres, the composition of the solid was tested and confirmed using SEM and the size of the spheres was determined using SEM and size measurements.

\subsubsection{UV-Vis Absorption Spectroscopy}

As seen in Figure 3.1, the UV-Vis absorption spectrum of prepared silica colloid shows a broad absorbance across the visible region, decreasing toward longer wavelength. This shows that silica has the potential to serve as a useful core material for these core-shell particles as the silica itself does not have any absorbance bands likely to interfere with the observation of any SPR bands due to the gold shells. 


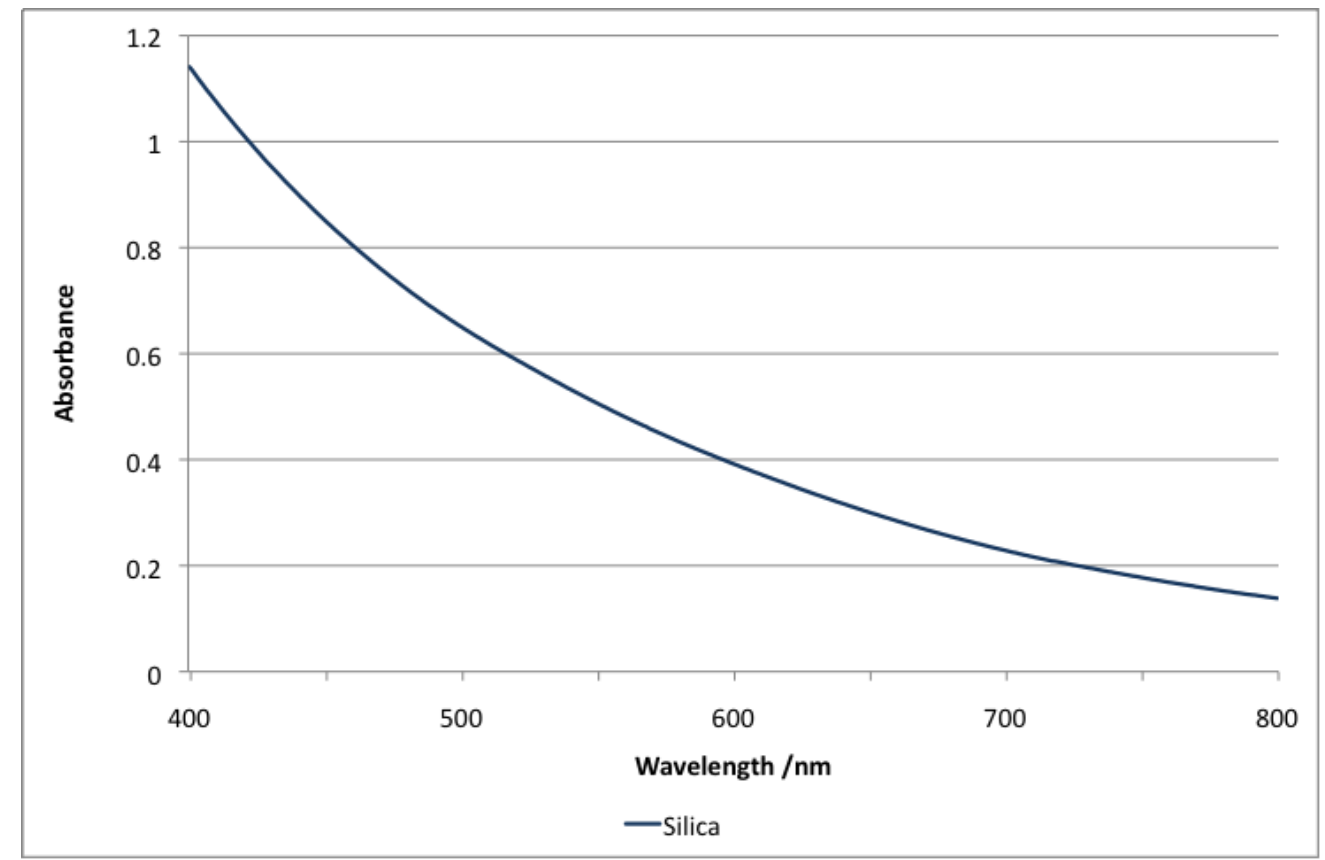

Figure 3.1: UV-Vis absorption spectrum of prepared silica spheres.

\subsubsection{SEM}

SEM imaging was used to determine the size and shape of the particles formed. SEM imaging was carried out on the JEOL-JSM- 6610LA instrument, and the samples were coated with $8 \mathrm{~nm}$ of carbon. As seen in Figure 3.2, images showed that the sample was reasonably monodisperse with particle diameter of approximately $250 \mathrm{~nm}$. 


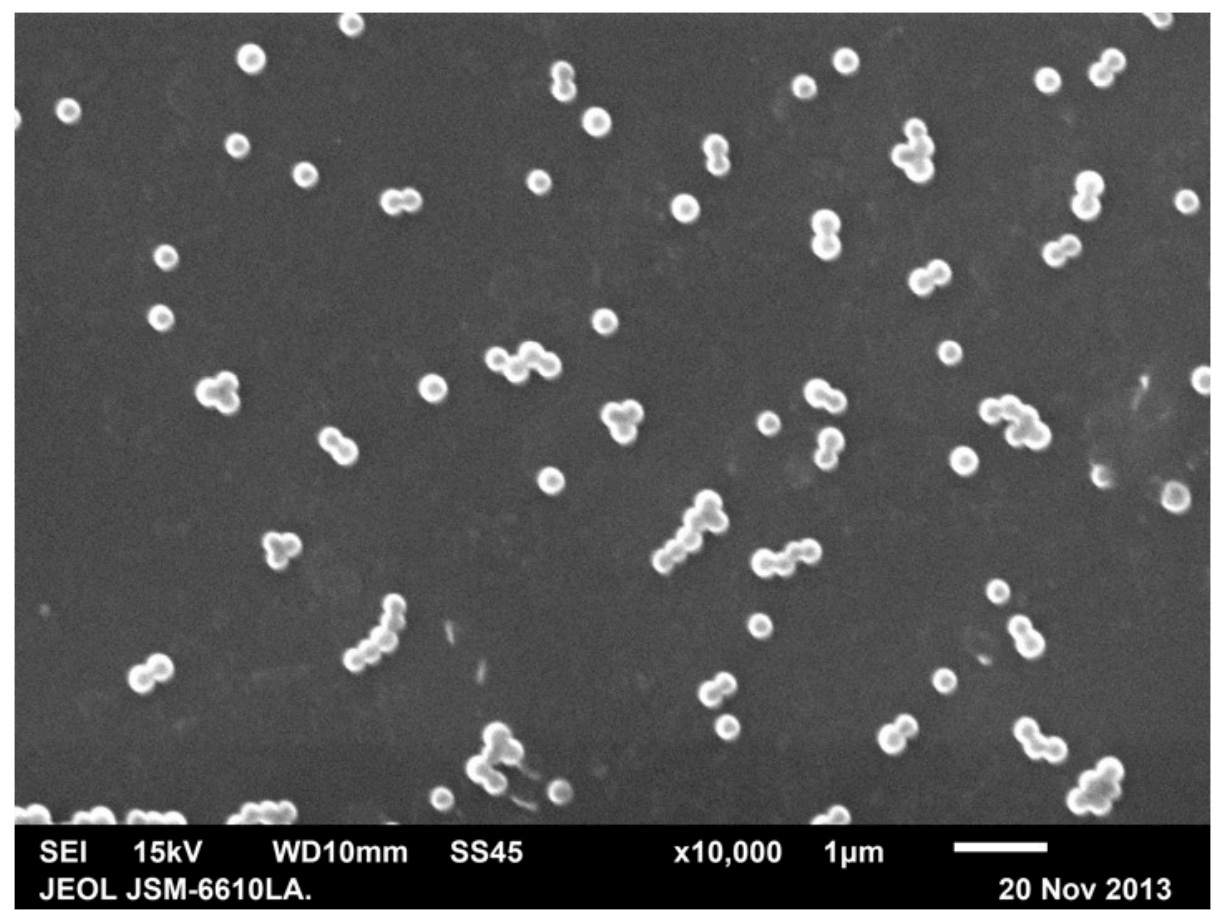

Figure 3.2: SEM image of synthesised silica particles showing size and monodispersity.

\subsubsection{Size Measurements}

Dynamic light scattering size measurements of the prepared silica were obtained using a Zeta-sizer. This method of size measurement determined the silica particles to be of $186 \mathrm{~nm}$ diameter, with very good monodispersity, as shown in Figure 3.3. The measurement obtained using this method slightly disagrees with observations from SEM imaging. Due to the different methods used, dynamic light scattering size measurements have a tendency to disagree with SEM measurements; dynamic light scattering measurements can include surface adsorbed molecules. However, this method is important as a source of evidence to support sizes determined by SEM and to show the polydispersity of the sample. 


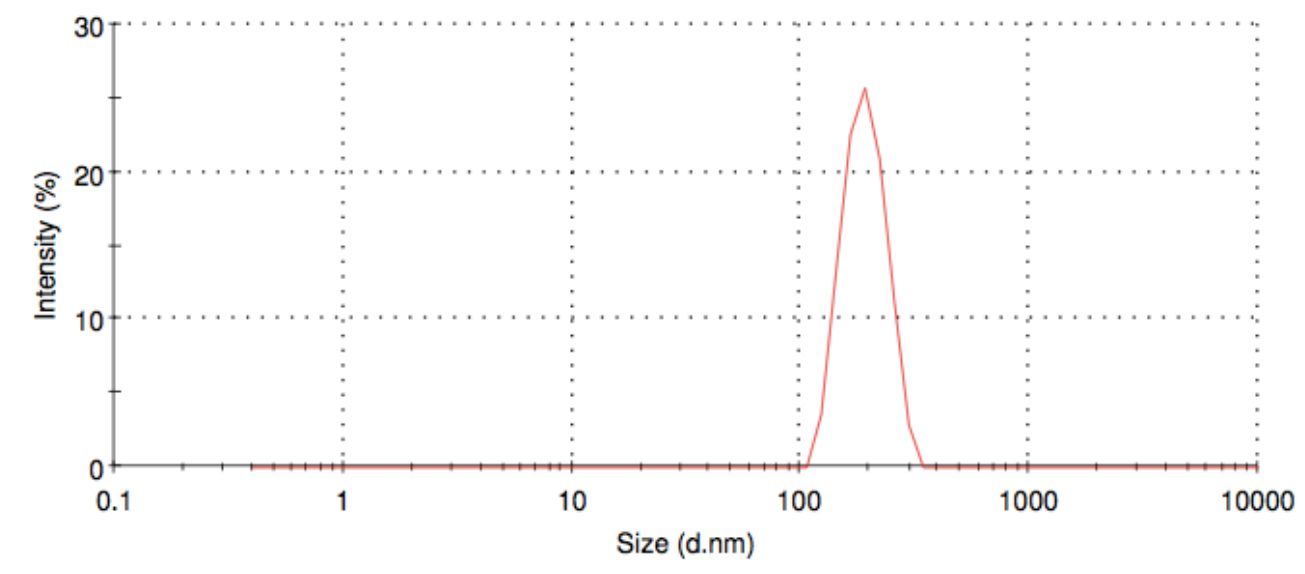

Figure 3.3: Size distribution of particles in prepared silica colloid showing average of $186 \mathrm{~nm}$ and good monodispersity.

\subsection{PEI Capped Silica Spheres}

Four samples of PEI capped silica spheres were prepared by adding PEI solution to silica spheres in water (see Section 2.2.2 PEI Capped Silica Spheres). Three samples were prepared in the same way and will be referred to by the wt $\%$ of PEI solution used in the reaction, while the fourth sample used a slightly different method and will be referred to by the label SP. The success and degree of capping was investigated using SEM imaging, EDS mapping and size measurements.

\subsubsection{UV-Vis Absorption Spectroscopy}

As shown in Figure 3.4, the UV-Vis absorption spectra of PEI capped silica spheres shows broad absorbance across the visible region. This broad absorbance decreases toward longer wavelength and appears to peak below $400 \mathrm{~nm}$. The absorbance is increased in the PEI capped silica compared to the uncapped silica due to the high absorbance of PEI itself, as shown in Figure 3.5. In the fourth sample, SP, which was synthesised in a different way, this absorbance decreases faster than the other samples, hence the absorption characteristics of this colloid are likely to interfere less with the anticipated SPR absorption bands of gold shells. 


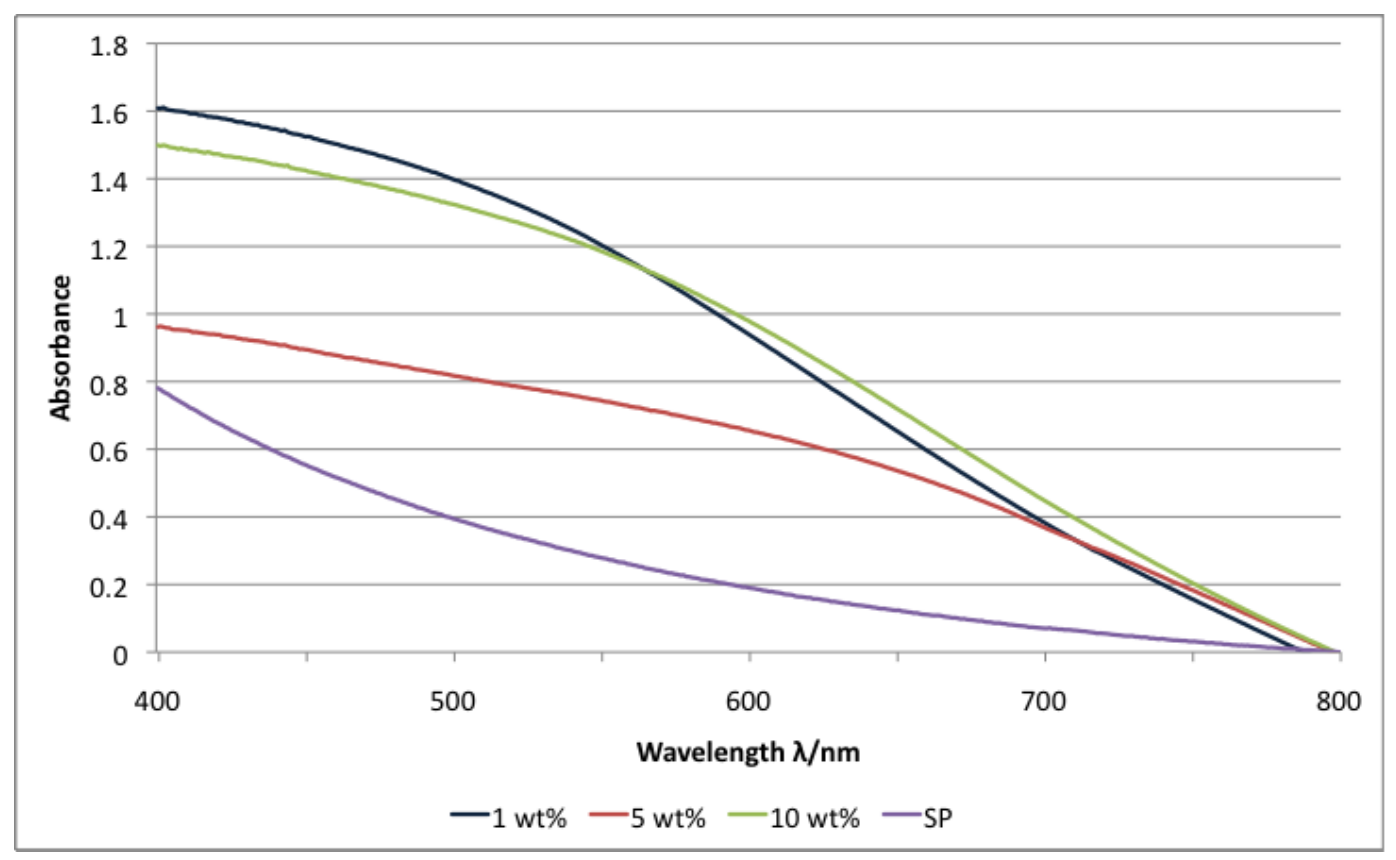

Figure 3.4: UV-Vis absorption spectra of PEI capped silica spheres.

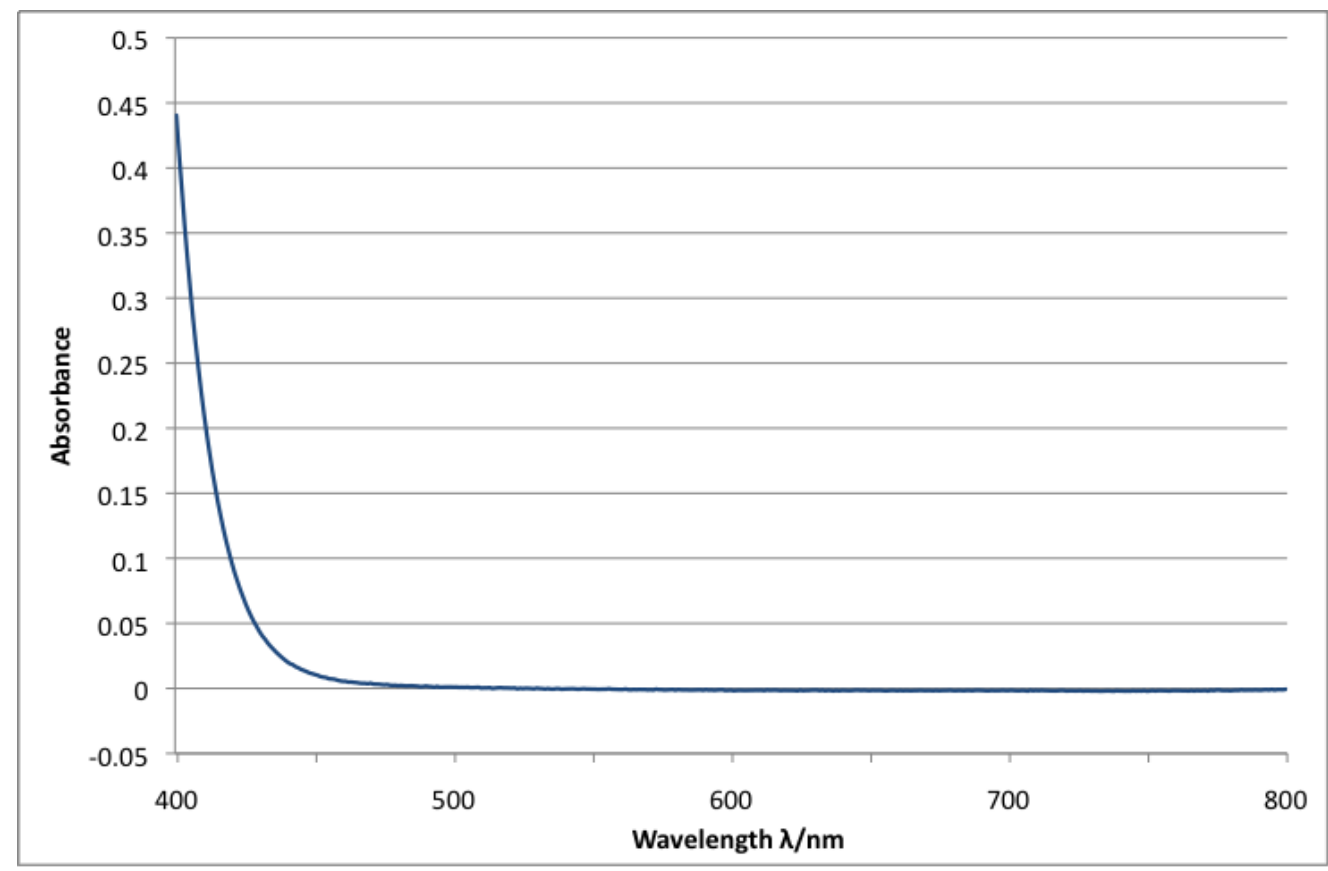

Figure 3.5: UV-Vis absorption spectrum of a 5 wt\% PEI solution in water. 


\subsubsection{SEM}

SEM imaging was used to determine the size and shape of silica spheres after capping with PEI. SEM imaging was carried out on the JEOL-JSM-6610LA instrument, and samples were coated with $8 \mathrm{~nm}$ of carbon. As seen in Figure 3.6, images showed that the silica spheres retained their monodispersity and were approximately $200 \mathrm{~nm}$ in diameter. This is a smaller diameter than that measured for uncapped silica spheres using SEM imaging. The Ostwald ripening and aggregation of the uncapped silica can explain this difference in size. As the SEM imaging was performed several days after the samples were prepared, the uncapped silica samples were left standing without a stabiliser, meaning aggregation and Ostwald ripening were free to occur; this caused the average particle size to increase in that colloid. In contrast, the particles in the colloids of capped silica particles were stabilised and Ostwald ripening was prevented. The use of backscatter mode on the SEM allowed investigation into the extent of the capping. Backscatter imaging shows higher atomic number (higher density) material as a brighter signal, hence in the backscatter images where there is a darker area surrounding the particles, it can be interpreted that there is a full layer of capping agent present on each of the silica spheres. 

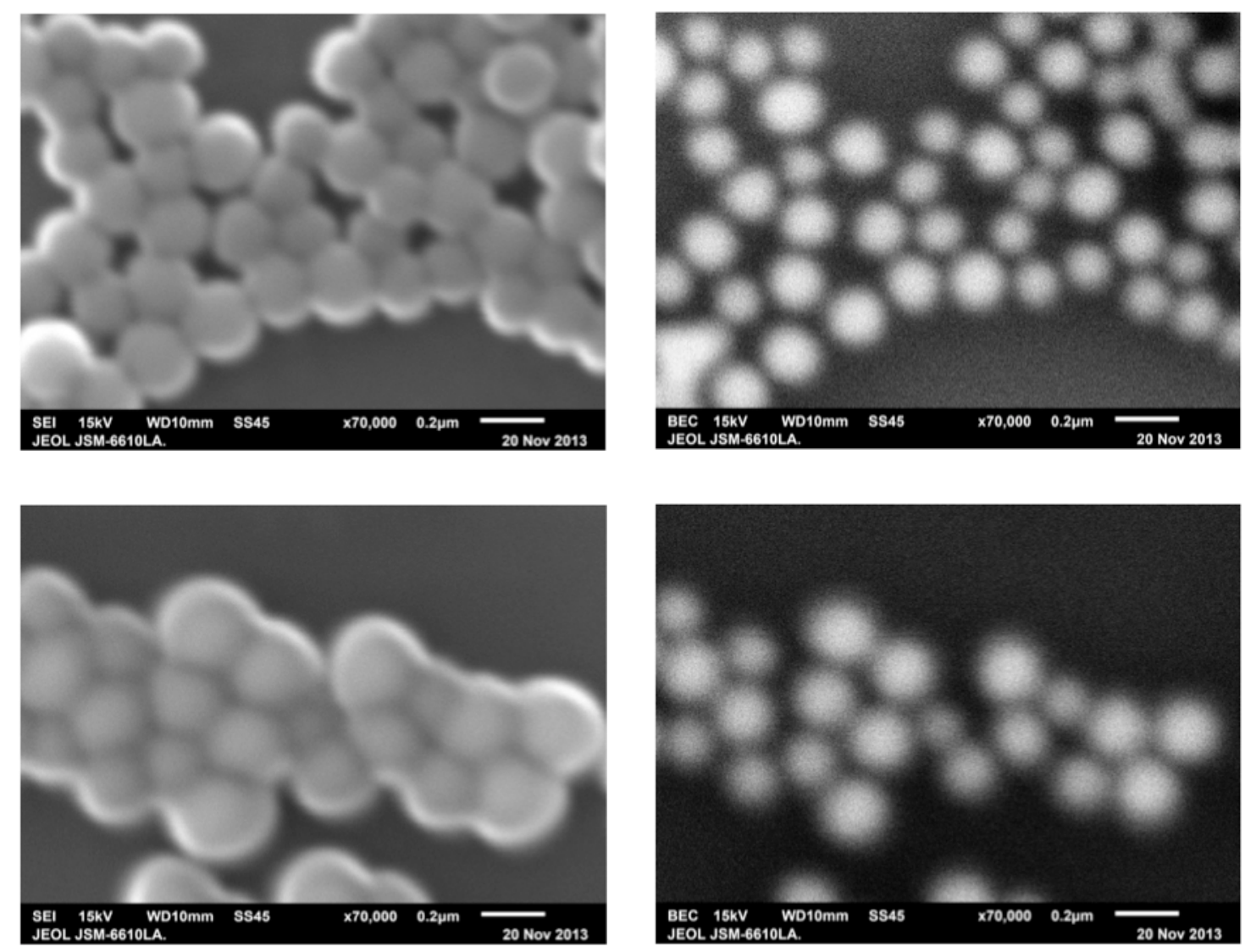

Figure 3.6: SEM images of PEI capped silica spheres (10 wt\% - top and SP - bottom samples) in both SEI (left) and backscatter (right) modes. 


\subsubsection{Size Measurements}

Dynamic light scattering size measurements of the four samples of silica spheres capped with PEI were obtained using a Zeta-sizer. The results of these measurements are shown in Figure 3.7 and summarised in Table 3.1. The three samples prepared in a similar fashion using differing wt $\%$ of PEI solutions all had a hugely increased particle diameter compared to the uncapped silica and poor monodispersity, shown by a broad peak in the particle size distribution. The particle size distribution showed a second peak at higher particle diameter. This could be due to contaminants such as dust. Hence the average diameter presented below is that of the first peak only. The fourth sample had a smaller diameter and much better monodispersity, shown by a sharper peak in the particle size distribution. No second peak was seen in the particle size distribution for this sample.

Table 3.1: Size measurements of diameters of silica spheres capped with PEI.

$\begin{array}{ccc}\text { Sample } & \text { Average diameter }(\mathrm{nm}) & \text { Monodispersity } \\ 1 \mathrm{wt} \% & 379 & \text { Poor } \\ 5 \mathrm{wt} \% & 544 & \text { Very Poor } \\ 10 \mathrm{wt} \% & 422 & \text { Poor } \\ \mathrm{SP} & 270 & \text { Very Good }\end{array}$



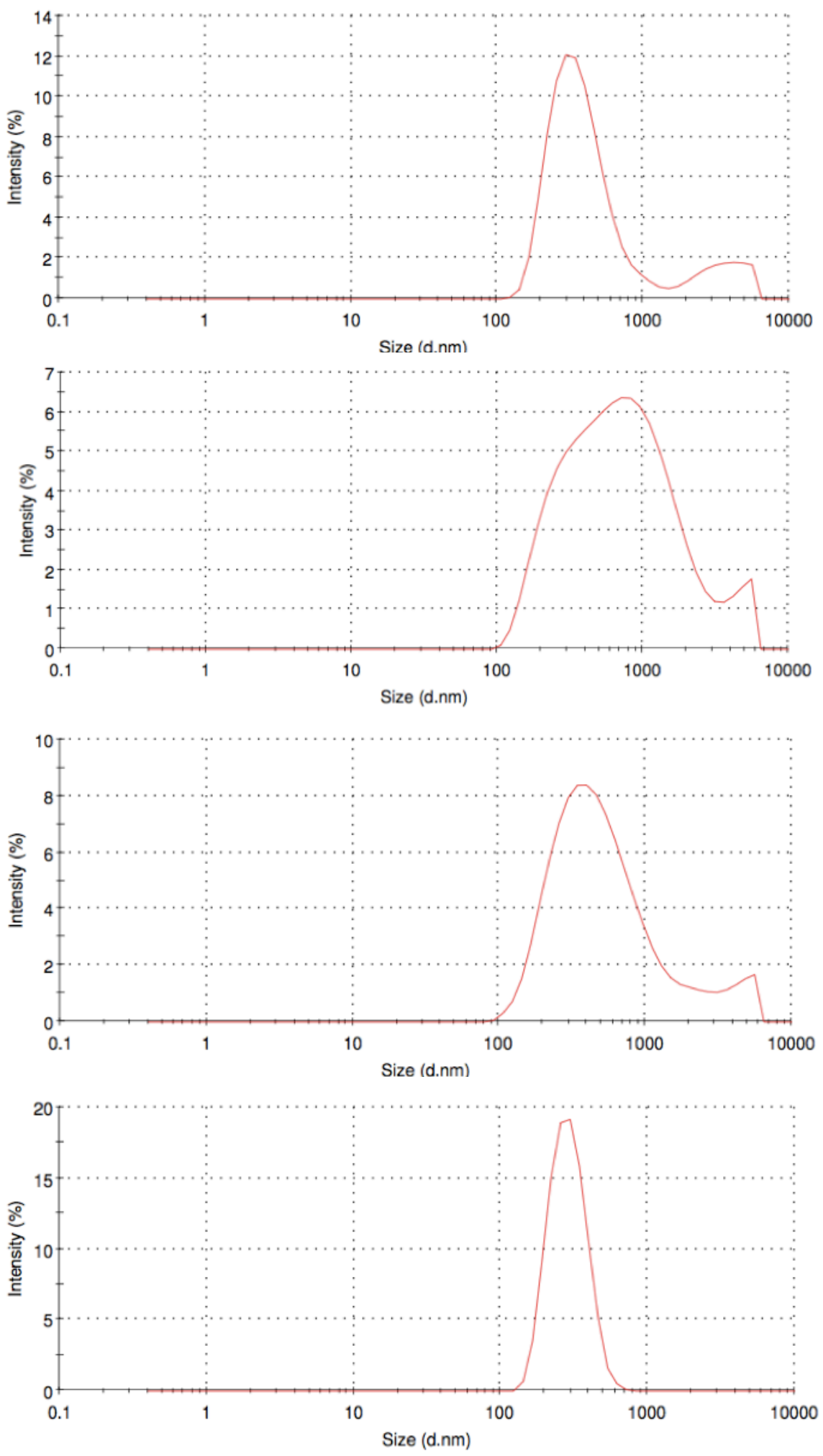

Figure 3.7: Size distributions of prepared PEI capped silica sphere colloids showing polydispersity and average particle size. Top to bottom: 1 wt $\%, 5 w t \%, 10 w t \%, S P$. 


\subsection{MPTMS Capped Silica Spheres}

MPTMS capped silica spheres were prepared by adding MPTMS to silica spheres (see Section 2.2.3 MPTMS Capped Silica Spheres). The success and degree of capping with MPTMS was investigated using, SEM imaging, EDS mapping and size measurements.

\subsubsection{UV-Vis Absorption Spectroscopy}

As shown in Figure 3.8, the UV-Vis absorption spectrum of MPTMS capped silica shows a broad absorption across the visible region. This absorption decreases toward longer wavelength. Hence the absorption behaviour of these MPTMS capped silica spheres are not anticipated to interfere with the observation of SPR absorption bands of gold shells. MPTMS solution itself does not show any absorbance in the visible region 400-800 $\mathrm{nm}$.

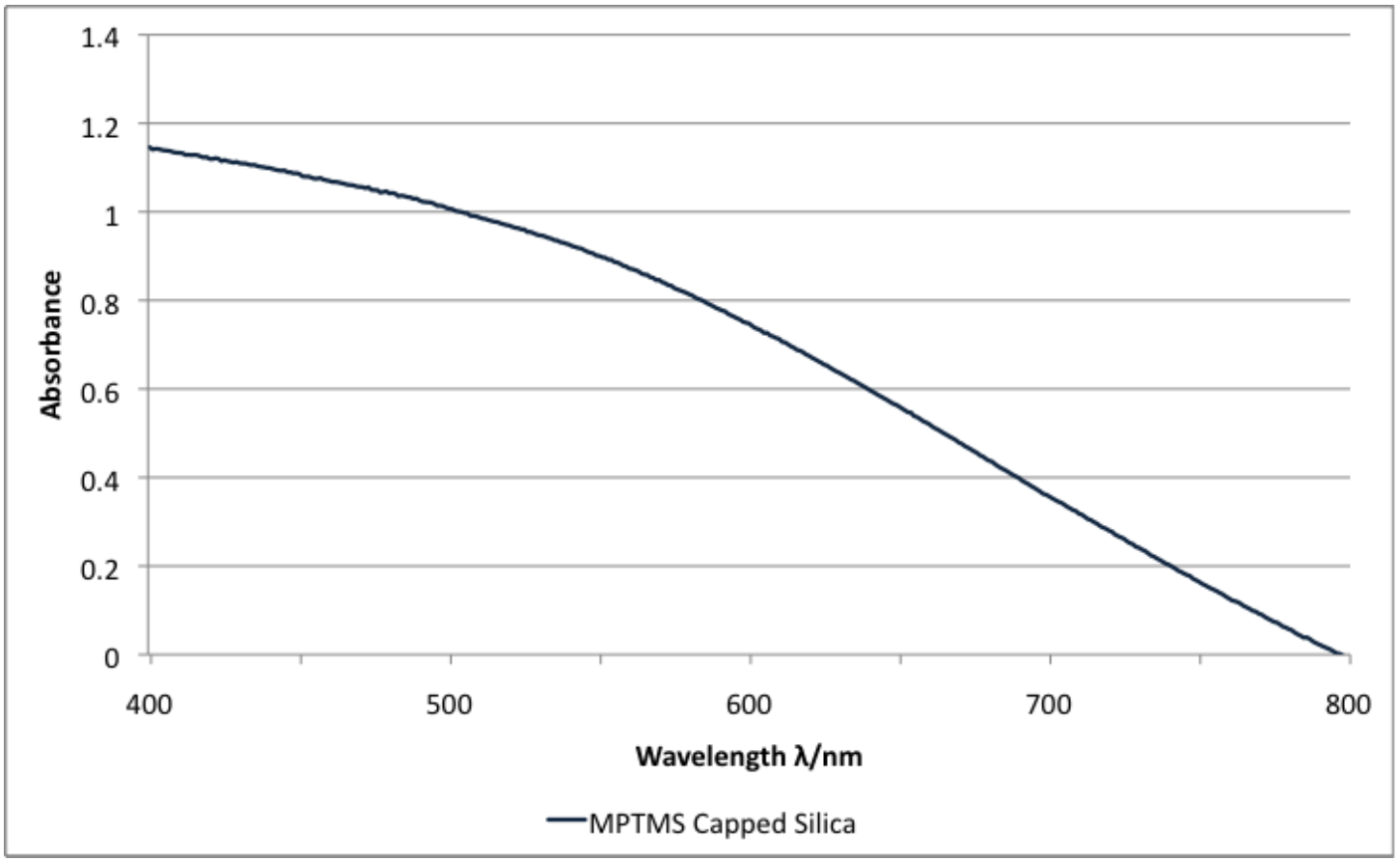

Figure 3.8: UV-Vis absorption spectrum of MPTMS capped silica spheres. 


\subsubsection{SEM}

SEM imaging was again used to determine the size and shape of silica spheres after capping with MPTMS. SEM imaging was carried out on the JEOL-JSM-6610LA instrument, and samples were coated with $8 \mathrm{~nm}$ of carbon. As seen in Figure 3.9, images show that the MPTMS capped silica spheres retained the monodispersity of uncapped silica spheres and were approximately $200 \mathrm{~nm}$ in diameter. Again, this is a smaller diameter than that measured for uncapped silica spheres by SEM imaging; this difference in diameter between uncapped and capped silica spheres can be explained by aggregation and Ostwald ripening of the unstabilised uncapped silica spheres. Backscatter imaging again showed that each silica sphere had a complete capping layer of MPTMS, shown as a dark area surrounding each sphere.
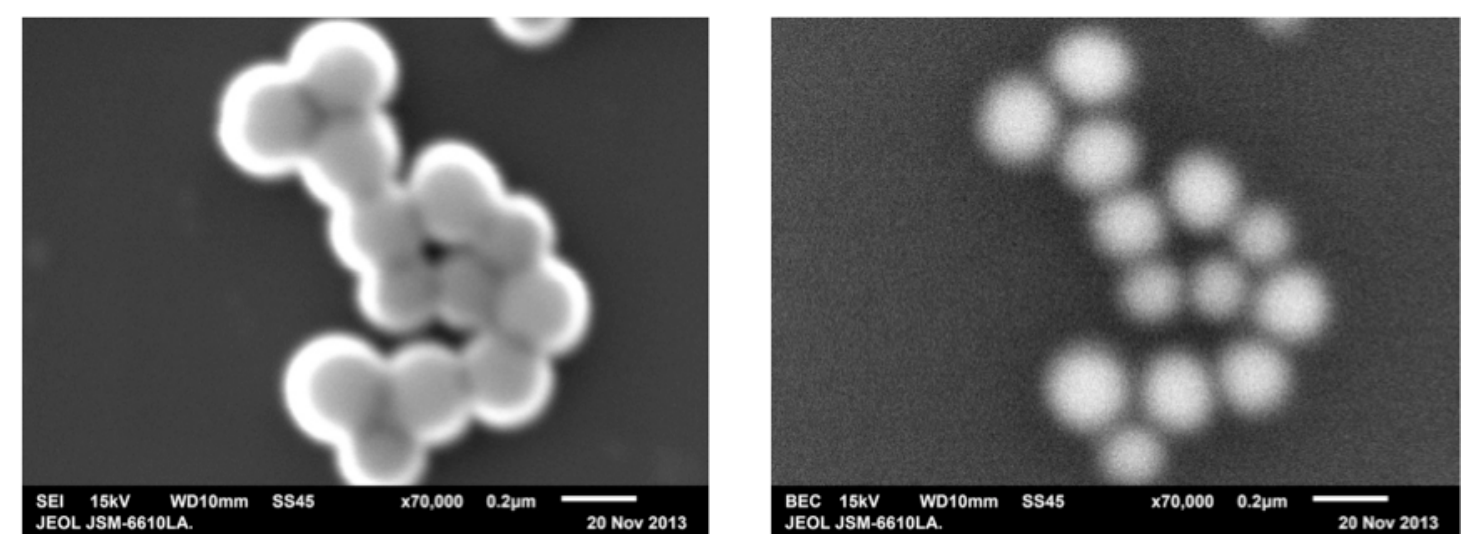

Figure 3.9: SEM images of silica spheres capped with MPTMS in both SEI (left) and backscatter (right) modes.

\subsubsection{Size Measurements}

Dynamic light scattering size measurements of the silica spheres capped with MPTMS were obtained using a Zeta-sizer. This method of size measurement determined the silica particles to be of $252 \mathrm{~nm}$ diameter, with good monodispersity, as seen in Figure 3.10. 


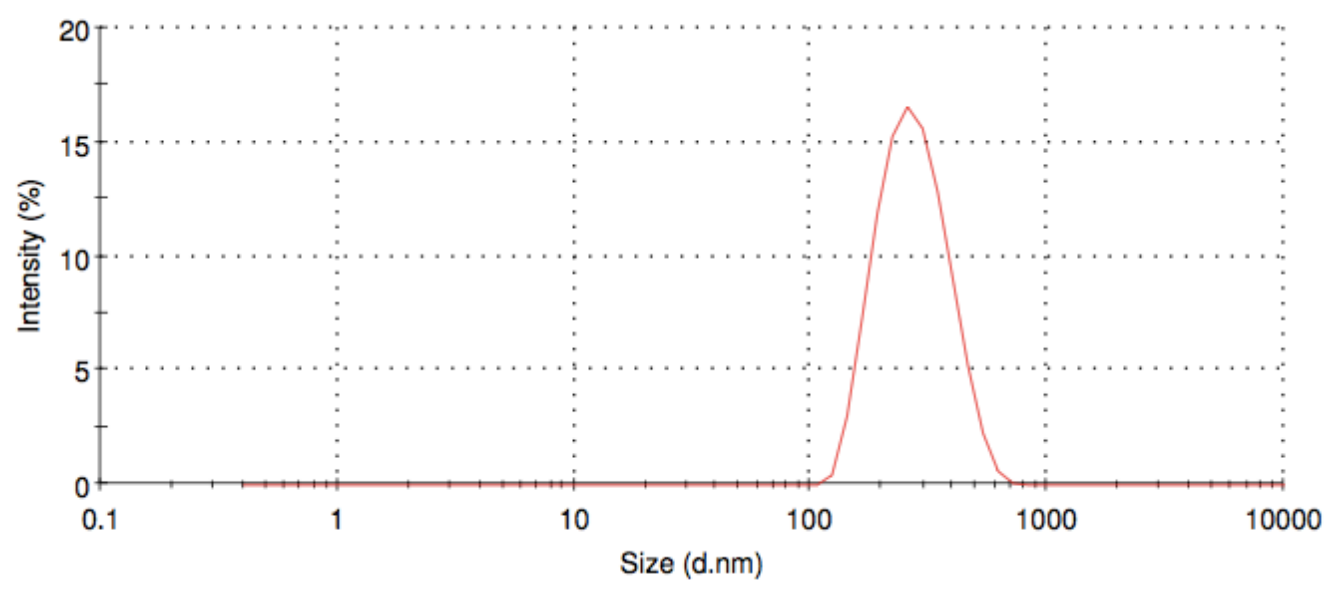

Figure 3.10: Size distribution of prepared MPTMS capped silica colloid showing monodispersity and average particle size.

\subsection{MPTMS Capped Titania Spheres}

Titania spheres were dispersed in water and MPTMS was added. This mixture was left shaking at $50{ }^{\circ} \mathrm{C}$ for 7 days (see Section 2.3.1 Titania Spheres Capped with MPTMS). During this time, the sample became inhomogeneous and the titania appeared to agglomerate and adhere to the sides of the vial. The capping of titania using MPTMS was deemed unsuccessful and therefore the titania spheres were deemed to be unsuitable as substrates for gold shells. As a result, further reactions to synthesise gold shells were not attempted on these particles.

\subsection{Conclusions from Characterisation of Silica and Titania}

SEM and EDS analysis of prepared silica samples showed that the silica spheres were approximately $180 \mathrm{~nm}$ in diameter and were successfully capped with either PEI or MPTMS. The size measurements obtained from the Zeta-sizer instrument were found to be slightly larger, this is expected, as the method of measurement tends to overestimate particle size slightly. The successful capping of the spheres will allow for their use as substrates for gold shells. 
SEM imaging showed that the average particle diameter of the uncapped silica spheres was greater than that of the capped silica spheres (both PEI and MPTMS capped samples). This increase in size of the uncapped silica particles was suspected to be due to aggregation and Ostwald ripening of the unstabilised colloid, as the samples were left to stand for several days before SEM analysis was carried out. As the capping agents acted as stabilisers in the capped silica colloids, aggregation and Ostwald ripening was prevented so the final particle sizes measured with SEM were smaller for these samples. Further experiments analysing the colloids after varied standing times are necessary to confirm Ostwald ripening and aggregation. As Zetasizer measurements were taken soon after the samples were prepared, these measurements show the expected trend of the capped silica samples having greater average particle diameter, as the capped silica samples contained the same silica spheres with additional volume of the capping layers.

The attempted capping of titania with MPTMS led to an inhomogeneous sample with the titania agglomerating and adhering to the sides of the vial. The capping process was deemed unsuccessful, hence the titania was not considered a good substrate for gold shells and was not used in further reactions. 


\section{Characterisation of Gold Shells}

This chapter discusses the results of all gold shell syntheses investigated. This includes characterisation of samples produced and discussion on the outcome of each synthetic scheme investigated in this research.

\subsection{Ex-situ Seeding on PEI Capped Silica Spheres}

Ex-situ seeding on PEI capped silica spheres was investigated in order to compare the resulting gold shells with those obtained using methods involving in-situ seeding steps.

Samples were prepared by adding $1 \mathrm{~mL}$ of PEI capped silica to one of two gold colloids (A and B), made up to $5 \mathrm{~mL}$ and left on a shaker table at room temperature overnight. See Section 2.4.1 Ex-situ Seeding on PEI Capped Silica Spheres and Table 4.1 for full experimental details. 
Table 4.1: Samples prepared via ex-situ seeding on PEI capped silica spheres. PEI column denotes what concentration of PEI solution was used in the capping of the silica spheres (see Section 2.2.2 PEI Capped Silica Spheres).

\begin{tabular}{|c|c|c|c|}
\hline Sample name & $P E I$ & $\begin{array}{l}\text { Gold } \\
\text { colloid }\end{array}$ & $\begin{array}{l}\text { Volume of gold colloid } \\
(m L)\end{array}$ \\
\hline 01-A005 & $1 \mathrm{wt} \%$ & A & 0.5 \\
\hline 01-A01 & $1 \mathrm{wt} \%$ & A & 1.0 \\
\hline 01-B01 & $1 \mathrm{wt} \%$ & B & 1.0 \\
\hline 01-B02 & $1 \mathrm{wt} \%$ & B & 2.0 \\
\hline 05-A005 & $5 \mathrm{wt} \%$ & A & 0.5 \\
\hline 05-A01 & $5 \mathrm{wt} \%$ & A & 1.0 \\
\hline 05-B01 & $5 \mathrm{wt} \%$ & B & 1.0 \\
\hline 05-B02 & $5 \mathrm{wt} \%$ & B & 2.0 \\
\hline $10-\mathrm{A} 005$ & $10 \mathrm{wt} \%$ & A & 0.5 \\
\hline $10-\mathrm{A} 01$ & $10 \mathrm{wt} \%$ & A & 1.0 \\
\hline 10-B01 & $10 \mathrm{wt} \%$ & B & 1.0 \\
\hline 10-B02 & $10 \mathrm{wt} \%$ & B & 2.0 \\
\hline
\end{tabular}




\subsubsection{UV-Vis Absorption Spectroscopy}

UV-Vis absorption spectra were obtained for all prepared samples, as shown in Figure 4.1. It was seen that, for all PEI capped silica samples (01-, 05- and 10-), the -A01 samples showed two small absorption peaks in the visible region of the spectrum, at approximately $520 \mathrm{~nm}$ and $640 \mathrm{~nm}$. Due to the nature of the syntheses, it was expected that some spherical gold nanoparticles would be formed in the sample. The SPR absorption of these nanoparticles was expected to be seen at approximately $525 \mathrm{~nm}$, hence the first peak in these spectra was attributed to these spherical particles. SPR absorption of core-shell particles is expected to occur at higher wavelengths than discrete gold nanoparticles, therefore the higher wavelength peak, occurring at approximately $640 \mathrm{~nm}$ could be attributed to core-shell particles in the samples. The other samples showed only very small peaks and most only exhibited a single absorption peak in the visible region, which was deemed to be solely due to spherical gold nanoparticles in the sample. Therefore, all -A01 samples were submitted for further analysis, while all other samples were deemed unsuccessful. 


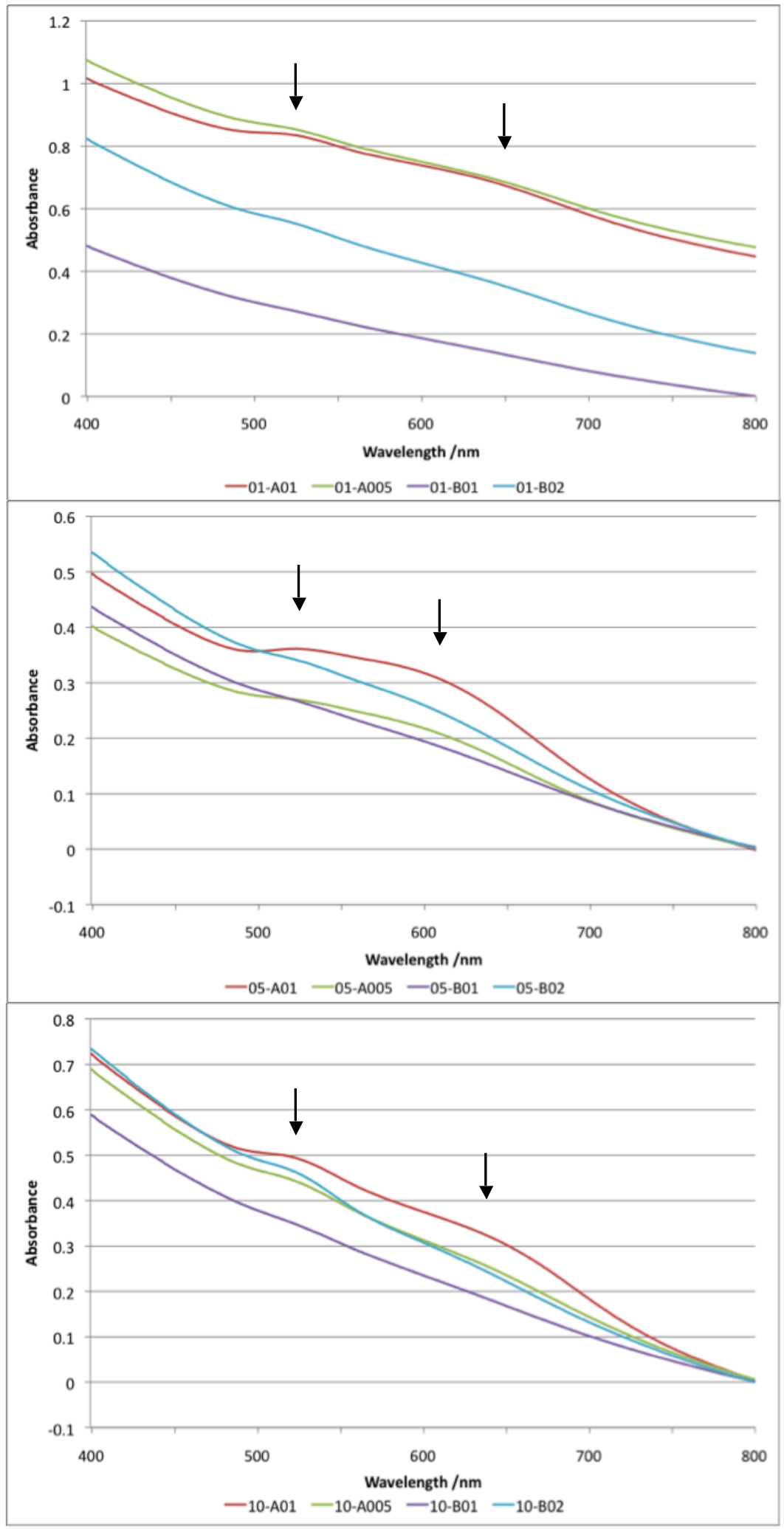

Figure 4.1: UV-Vis absorption spectra of ex-situ seeded gold shells on PEI capped silica spheres. The samples were not the same concentrations. 


\subsubsection{SEM}

SEM was carried out on the JEOL-JSM-6610LA instrument, and samples were coated with $8 \mathrm{~nm}$ of carbon. All -A01 samples were imaged and all gave similar results. As shown by Figure 4.2, it was found that the samples contained some discrete gold nanoparticles and some large aggregates. Backscatter imaging showed that these areas of gold were sometimes associated with the PEI capping layer, as they were correlated with the areas of lower density material surrounding the cores. However, although these gold particles and aggregates were somewhat associated with the PEI layer, no core-shell particles or partial shells were found to be present in any of the samples examined.
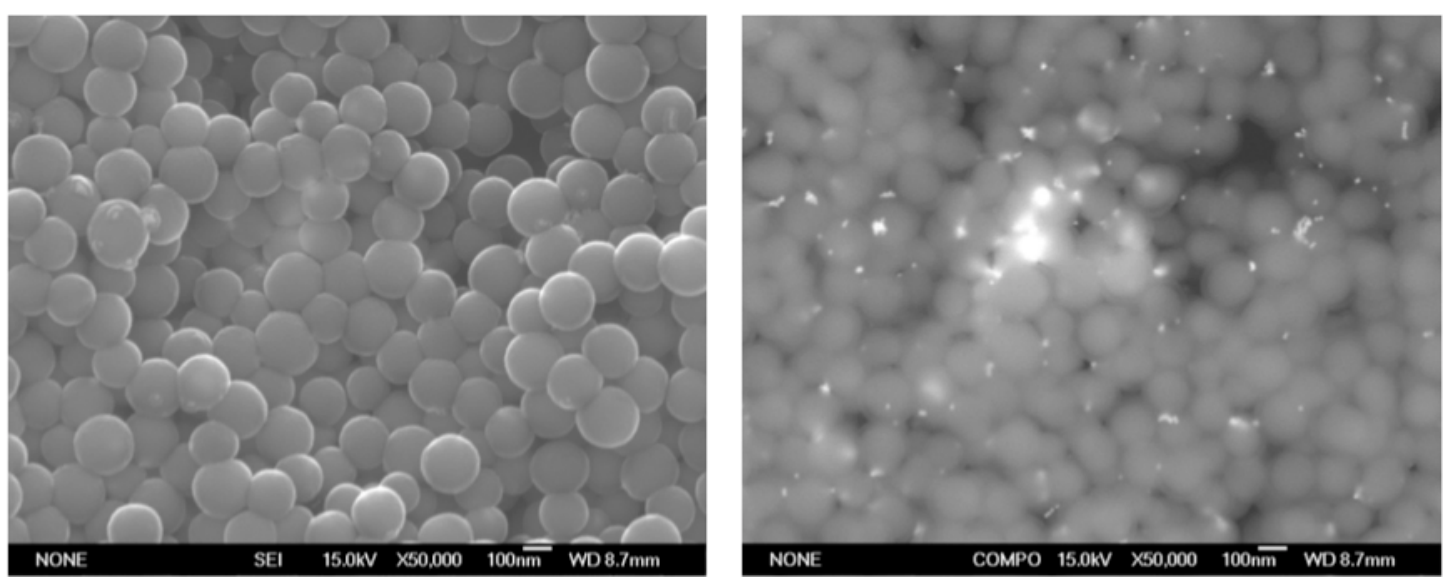

Figure 4.2: SEM images of Sample 10-A01 in both SEI (top) and backscatter (bottom) modes. In backscatter image, gold is shown as bright white areas.

\subsubsection{Summary}

The UV-Vis absorption spectra of the -A01 group of samples showed two peaks in the visible range; the first peak occurred at approximately $520 \mathrm{~nm}$, which was attributed to discrete spherical gold nanoparticles present in the sample. The second peak at $640 \mathrm{~nm}$ was suspected to be due to potential core-shell particles. SEM images showed that these samples contained discrete gold nanoparticles as well as many aggregates, however no core-shell particles were seen and these reactions were deemed unsuccessful. Hence, the higher wavelength peaks in the UV-Vis absorption 
spectra were determined to be due to gold aggregates rather than core-shell particles as first suspected.

\subsection{In-situ Seeding on PEI Capped Silica Spheres}

In-situ seeding on PEI capped silica spheres was investigated using only the capping layer of PEI as reductant or with additional PEI in solution as external reducing agent. These syntheses require only PEI, silica spheres and $\mathrm{AuCl}_{4}{ }^{-}$solution; hence they have fewer steps and fewer reagents than previously reported methods outlined in Section 1.3.1 Gold Shells.

Samples were prepared by adding PEI capped silica and $\mathrm{AuCl}_{4}{ }^{-}$together, with or without additional free PEI as an external reductant and left at $50{ }^{\circ} \mathrm{C}$ for 7 days, see Section 2.4.2 In-situ Seeding on PEI Capped Silica Spheres and Table 4.2 for full experimental details. 
Table 4.2: Samples prepared via in-situ seeding on PEI capped silica spheres. PEI column denotes what concentration of PEI solution was used in the capping of the silica spheres.

\begin{tabular}{|c|c|c|c|}
\hline Sample Name & $P E I$ & $\begin{array}{l}\text { Volume of } 100 \mathrm{ppm} \\
\mathrm{AuCl}_{4}^{-} \text {solution (mL) }\end{array}$ & $\begin{array}{c}\text { Volume of additional } \\
\qquad 1 w t \% \text { PEI (mL) }\end{array}$ \\
\hline 01-C01 & $1 \mathrm{wt} \%$ & 1.0 & 0.0 \\
\hline 01-C02 & $1 \mathrm{wt} \%$ & 2.0 & 0.0 \\
\hline 01-C01a & $1 \mathrm{wt} \%$ & 1.0 & 2.0 \\
\hline $01-\mathrm{C} 02 \mathrm{a}$ & $1 \mathrm{wt} \%$ & 2.0 & 2.0 \\
\hline 05-C01 & $5 \mathrm{wt} \%$ & 1.0 & 0.0 \\
\hline 05-C02 & $5 \mathrm{wt} \%$ & 2.0 & 0.0 \\
\hline 05-C01a & $5 \mathrm{wt} \%$ & 1.0 & 2.0 \\
\hline $05-\mathrm{C} 02 \mathrm{a}$ & $5 \mathrm{wt} \%$ & 2.0 & 2.0 \\
\hline $10-\mathrm{C} 01$ & $10 \mathrm{wt} \%$ & 1.0 & 0.0 \\
\hline $10-\mathrm{C} 02$ & $10 \mathrm{wt} \%$ & 2.0 & 0.0 \\
\hline $10-\mathrm{C} 01 \mathrm{a}$ & $10 \mathrm{wt} \%$ & 1.0 & 2.0 \\
\hline $10-\mathrm{C} 02 \mathrm{a}$ & $10 \mathrm{wt} \%$ & 2.0 & 2.0 \\
\hline
\end{tabular}




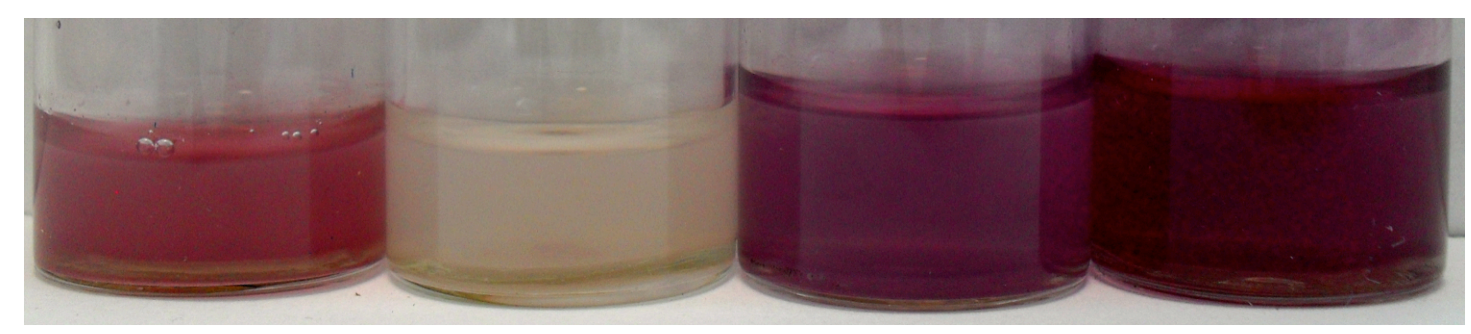

Figure 4.3: Photograph of samples 10-C01, 10-C02, 10-C01a and 10-C02a (left to right).

\subsubsection{UV-Vis Absorption Spectroscopy}

UV-Vis absorption spectra were obtained for all samples prepared. As shown in Figure 4.4, some samples presented one peak in the visible region. However these occurred at wavelengths typical of SPR absorption of spherical gold nanoparticles, not core-shell particles. No samples showed absorption peaks at higher wavelengths indicative of core-shell particles and so the reactions were deemed unsuccessful in synthesising the desired particles. 


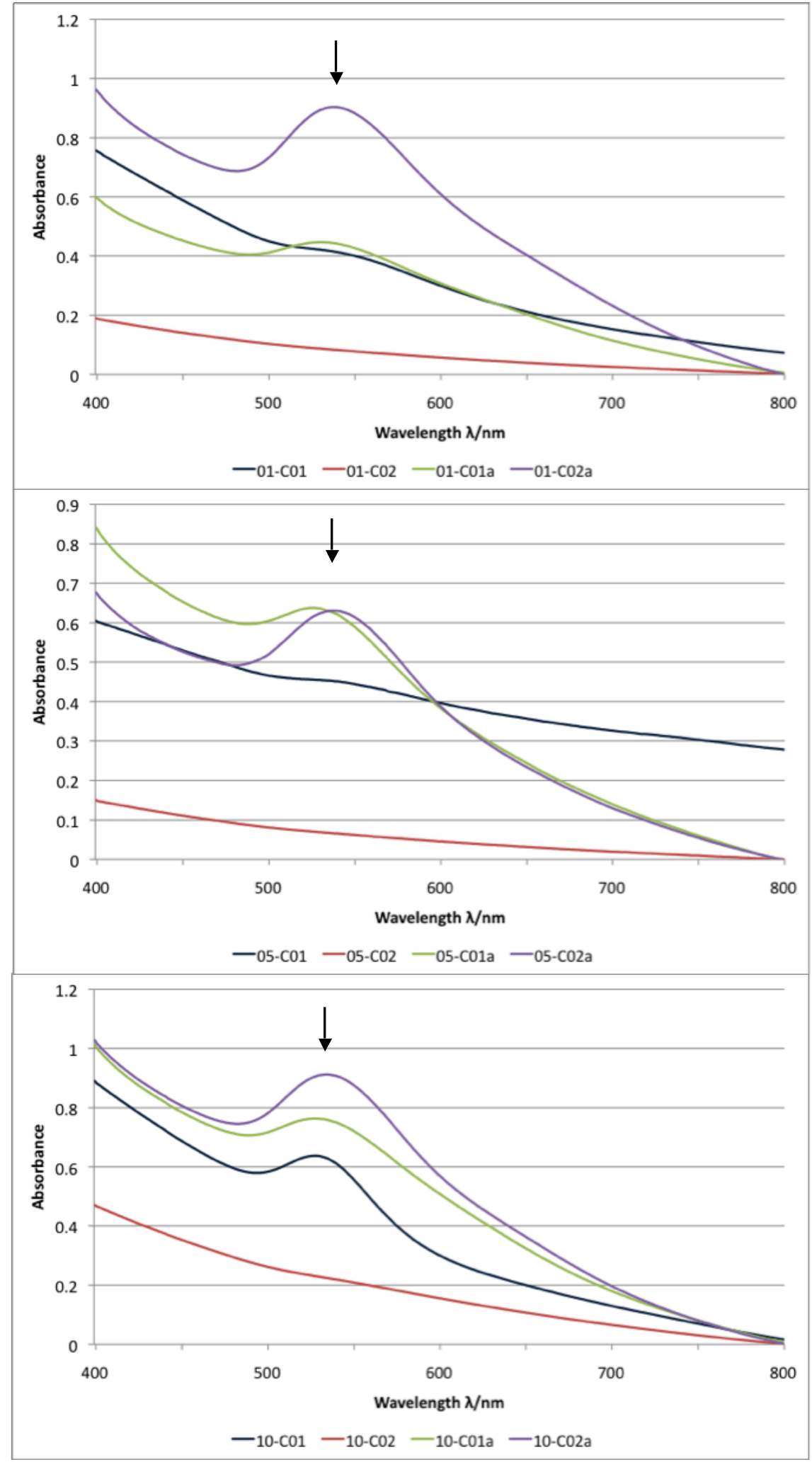

Figure 4.4: UV-Vis absorption spectra of samples prepared via in-situ seeding on PEI capped silica spheres. The samples were different concentrations. 


\subsubsection{SEM}

Sample 10-C01 was imaged on JEOL-6500F SEM, after being coated with $8 \mathrm{~nm}$ of carbon, and confirmed to have no core-shell particles in the sample. Instead, the sample contained groups of gold nanoparticles on the surface of the silica spheres. These gold nanoparticles, shown as bright areas in SEM images (Figure 4.5), were surrounded by a lower-density material, this is most likely PEI.

Previous literature has shown that the growth of discrete spherical gold nanoparticles can be an early stage in the synthesis of gold shells, as shown in Figure $1.15 .^{34}$ However, those gold nanoparticles are evenly distributed across the core surfaces, whereas in these reactions the gold nanoparticles are found in specific areas and are unevenly distributed across the silica sphere surfaces. Therefore, it is determined that these gold nanoparticles are not an early stage of potential gold shells.
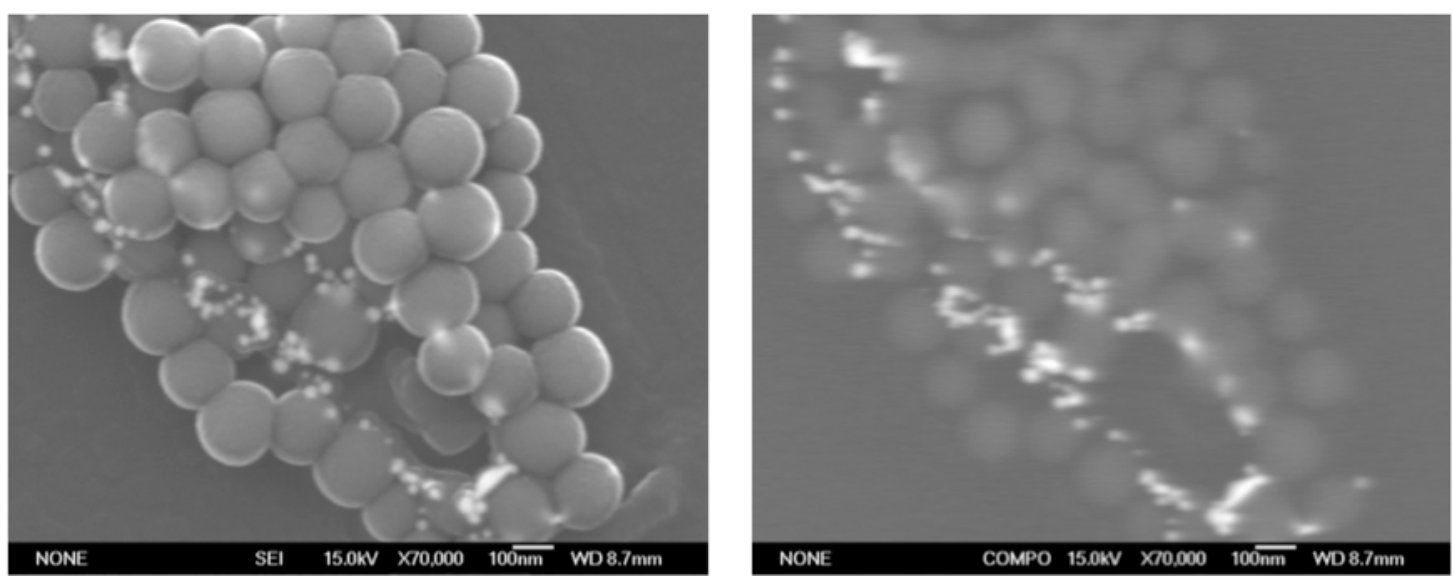

Figure 4.5: SEM images of sample 10-C01 in both SEI (left) and backscatter (right) modes.

\subsubsection{Summary}

Samples synthesised by these methods appeared similar colours to colloids of spherical gold nanoparticles and none of the UV-Vis absorption spectra of these samples showed peaks suggesting the formation of core-shell particles. SEM showed 
that core-shell particles were not synthesised, showing only discrete spherical gold nanoparticles. Hence, all syntheses were deemed unsuccessful.

\subsection{In-situ Seeding on PEI Capped Silica Spheres with Hydroxylamine}

It was found that in-situ seeding on PEI capped silica spheres without additional reducing agents was unsuccessful in producing the desired core-shell particles. Here, the use of the additional reducing agent hydroxylamine is investigated. Hydroxylamine is a weak reductant and will only reduce gold onto a surface that already has gold on it. Hence, the synthesis still relies on the PEI capping layer for the initial seeding of gold nanoparticles on the surface.

The sample (sample name PGb) was prepared by adding $\mathrm{AuCl}_{4}{ }^{-}$to PEI capped silica, followed by addition of hydroxylamine solution, and 3 further additions of $\mathrm{AuCl}_{4}^{-}$, see Section 2.4.3 In-situ Seeding on PEI Capped Silica Spheres with Hydroxylamine. During the synthesis, the yellow colour of $\mathrm{AuCl}_{4}{ }^{-}$was lost, but no colour was developed and the sample remained colourless with white precipitate. This indicated that core-shell particles had not been formed. This was investigated with UV-Vis absorption spectroscopy, which confirmed that the sample had no absorption peaks in the visible region.

\subsubsection{UV-Vis Absorption Spectroscopy}

As shown in Figure 4.6, UV-Vis absorption spectroscopy showed that there was no absorption in the visible region. This confirms the failure to synthesise coreshell particles in this sample. The lack of any absorption peak indicates that spherical gold nanoparticles were not formed, indicating that the PEI capping agent was not effective in reducing $\mathrm{AuCl}_{4}{ }^{-}$to gold metal for the initial gold nanoparticle seeds. 


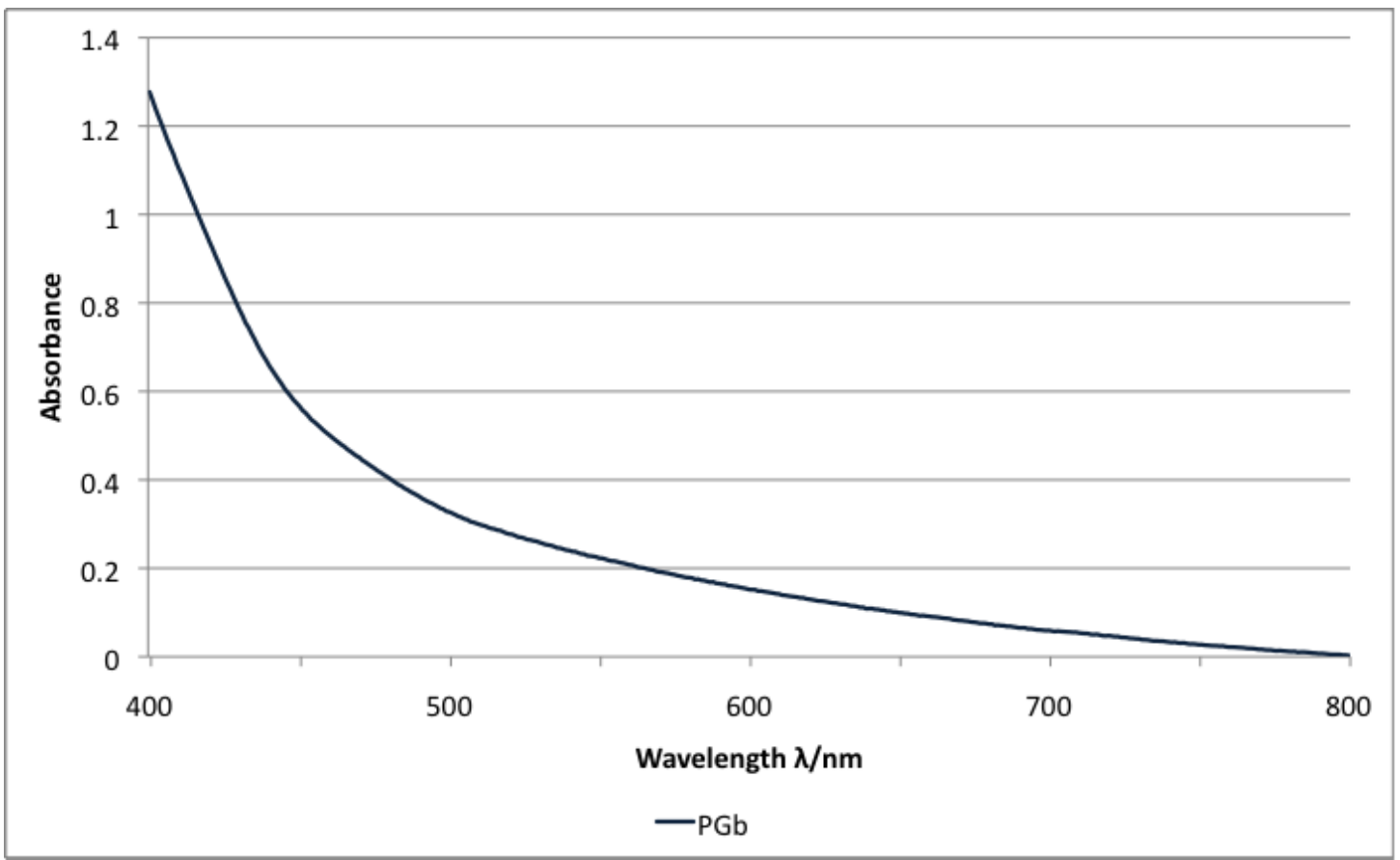

Figure 4.6: UV-Vis absorption spectrum of PGb showing no absorption peak in the visible region.

\subsection{In-situ Seeding on MPTMS Capped Silica}

\section{Spheres}

It was determined that the PEI capping agent was not effective in reducing $\mathrm{AuCl}_{4}{ }^{-}$to gold metal in the initial in-situ seeding. Here, the use of MPTMS as a capping agent is investigated. These first syntheses investigated with MPTMS capped silica spheres involved only $\mathrm{AuCl}_{4}{ }^{-}$and MPTMS capped silica in water as solvent. These gold shell syntheses require higher temperatures, however they involve very few steps and only two reagents.

Samples were prepared by adding $\mathrm{AuCl}_{4}{ }^{-}$solution to $1 \mathrm{~mL}$ of $1 \mathrm{wt} \% \mathrm{MPTMS}$ capped silica spheres in $19 \mathrm{~mL}$ of water and heating for 2 hours, see Section 2.4.4 In-situ Seeding on MPTMS Capped Silica Spheres. 
Table 4.3: Samples prepared via in-situ seeding on MPTMS capped silica particles.

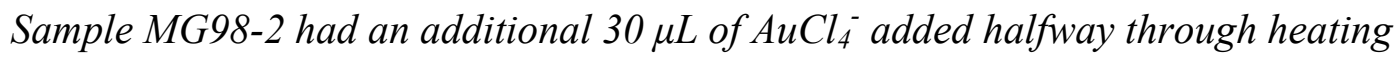
time (after 1 hour).

Sample name

Temperature $\left({ }^{\circ} \mathrm{C}\right)$

Volume of $\mathrm{AuCl}_{4}^{-}(\mu L)$

MG98-1

98

50

MG98-2

98

$50+30$

MG98-3

98

80

MG75

75

50

Within 30 minutes, the colour of the samples changed. MG98-1 and MG98-2 turned quite dark orange/brown (slightly purple in transmitted light), whereas MG98-3 turned a similar colour but much lighter. MG98-3 also had some large precipitate particles after 1 hour. After the second addition of $\mathrm{AuCl}_{4}^{-}(30 \mu \mathrm{L})$ to MG98-2 the sample became slightly darker. MG75 lost the yellow colour during heating but only developed a slight orange/purple colour that appeared after 2 hours of heating. After standing at room temperature for a few days, MG98-1 noticeably had a much more intense colour than the other three samples, MG98-2 had lost most of its colour. Final colours of samples are shown in Figure 4.7. 


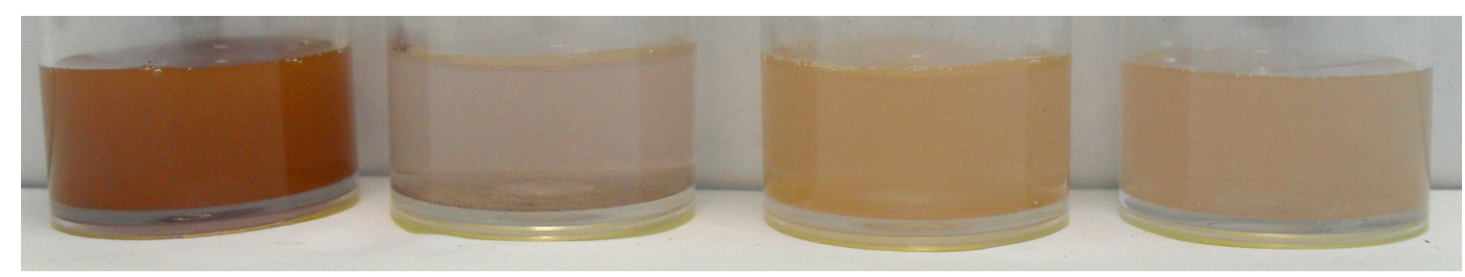

Figure 4.7: Photograph of samples MG98-1, MG98-2, MG98-3 and MG75 (left to right).

It was observed that the samples appeared different colours in reflected light than in transmitted light, as demonstrated in Figure 4.8. This phenomenon is similar to that observed in the Lycurgus Cup. ${ }^{56}$ This is due to the nanogold components causing different wavelengths of light to be scattered upon reflection and transmission.
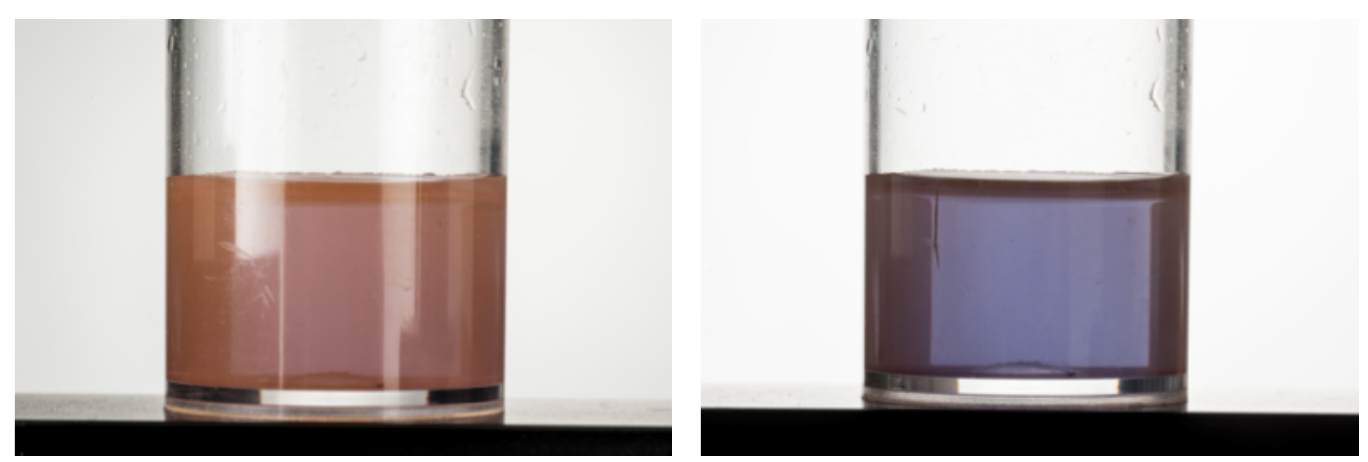

Figure 4.8: Photographs of sample MG98-1, demonstrating different observed colours in reflected (left) and transmitted (right) light. This same phenomenon occurred for all samples that appeared a similar brown/orange colour in reflected light. 


\subsubsection{UV-Vis Absorption Spectroscopy}

UV-Vis absorption spectra, seen in Figure 4.9, showed that samples exhibited one major absorption peak at approximately $550 \mathrm{~nm}$ and another small minor peak at approximately $710 \mathrm{~nm}$. The specific wavelengths of these absorption peaks are summarised in Table 4.4. The major peak observed at approximately $550 \mathrm{~nm}$ was deemed to be due to spherical gold nanoparticles present in the sample, while the smaller peak at higher wavelength was deemed to be indicative of core-shell particles. MG98-1 was not observed to exhibit this peak at higher wavelength. However it cannot be definitively stated that the peak is absent rather if it is present, it is too small to be detected above the background of the spectrum.

Table 4.4: Wavelengths of major and minor absorption bands of samples prepared via in-situ seeding on MPTMS capped silica spheres. MG98-1 did not present a significant minor peak.

Sample name

Major peak wavelength

$\left(\lambda_{\max } / n m\right)$
Minor peak wavelength

$$
\left(\lambda_{\max } / n m\right)
$$

MG98-1

MG98-2

MG98-3

MGB75 


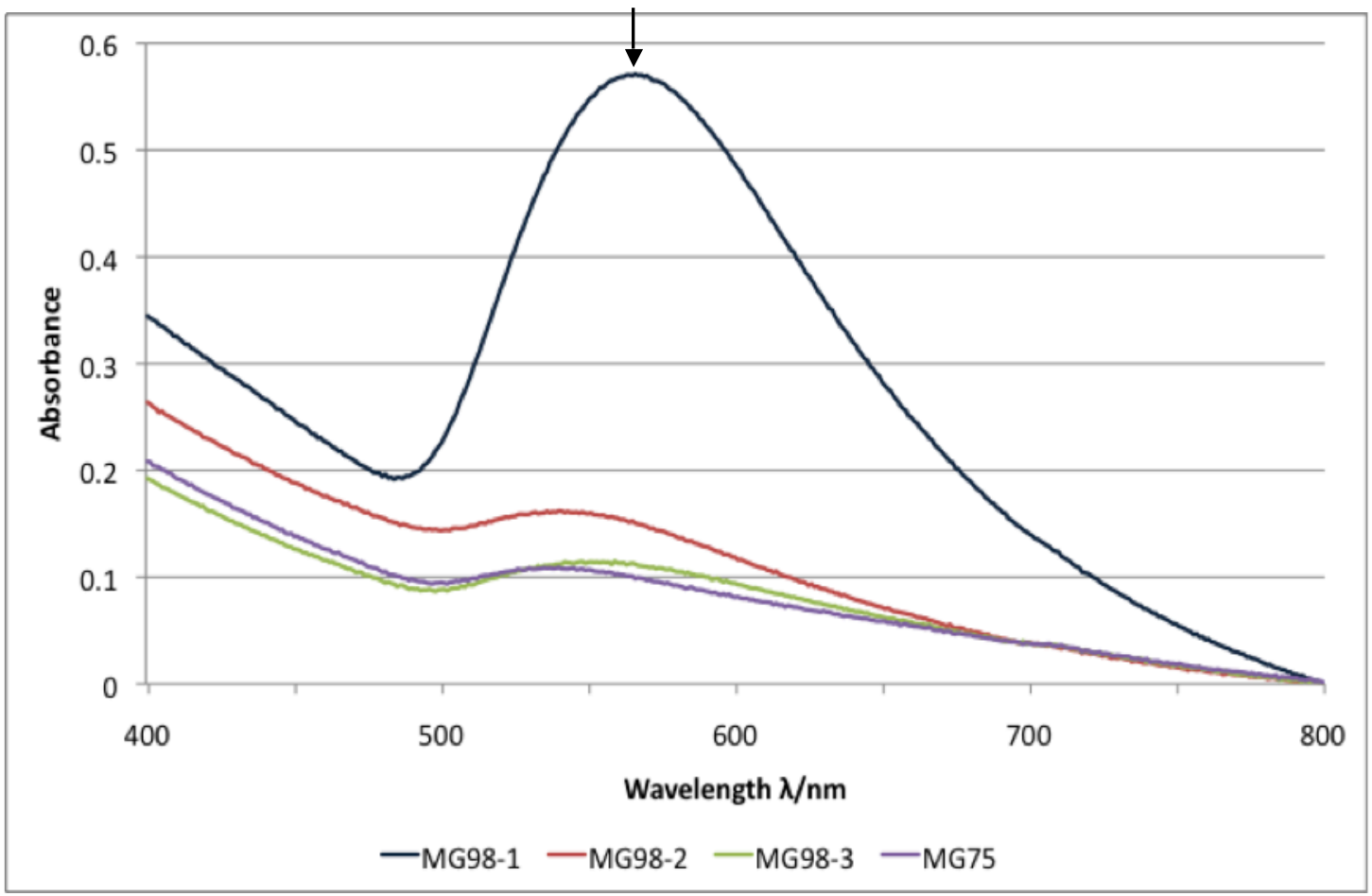

Figure 4.9: UV-Vis absorption spectra of samples prepared via in-situ seeding on MPTMS capped silica spheres. Samples were not of equal concentrations.

\subsubsection{SEM}

SEM was carried out on JEOL-JSM-6610LA instrument with samples coated with $4 \mathrm{~nm}$ of carbon. Samples prepared at $98{ }^{\circ} \mathrm{C}$ showed clumped areas of gold aggregated together with silica spheres, as seen in Figure 4.10. Some spherical particles of high-density material were present in the samples and determined to be core-shell particles using EDS analysis. These particles were considerably larger than the silica spheres, approximately $375 \mathrm{~nm}$ in diameter, hence the gold shells formed in these samples were rather thick. 


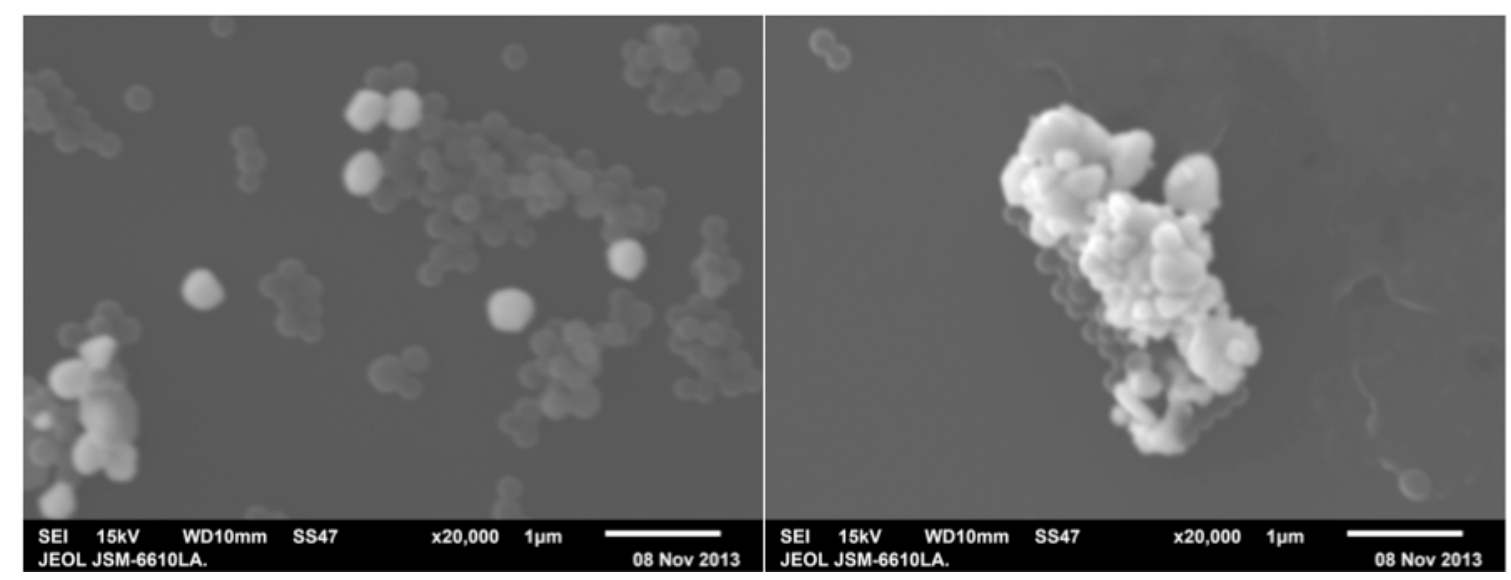

Figure 4.10: SEM images of sample MG98-3 showing large core-shell particles (left image) and of sample MG98-2 showing gold aggregated with silica spheres.

As seen in Figure 4.11, SEM imaging of MG75 showed some platelets of gold and some clumps of gold aggregated together with silica spheres. A few core-shell-like particles were found in the sample. EDS analysis, shown in Figure 4.12, confirmed the presence of oxygen in the same areas as these gold spheres, indicating they were indeed silica-gold core-shell particles.
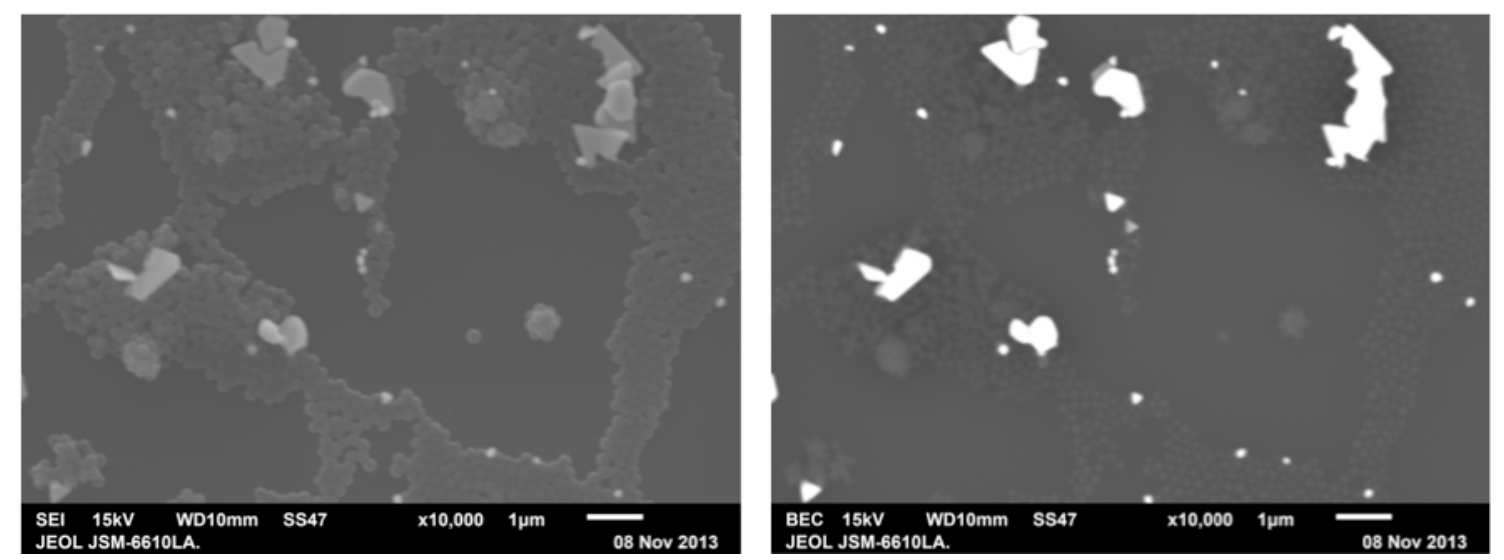

Figure 4.11: SEM images of sample MG75 in both SEI (left) and backscatter (right) modes showing small spherical gold particles, gold platelets and gold aggregates. 
EDS analysis was carried out on the samples to determine the elemental composition of the core-shell particles. Due to the thickness of the dense gold shells the oxygen signals from the silica cores were very weak and maps had to run for extended time periods to give a clear signal indicating the presence of oxygen in these areas.
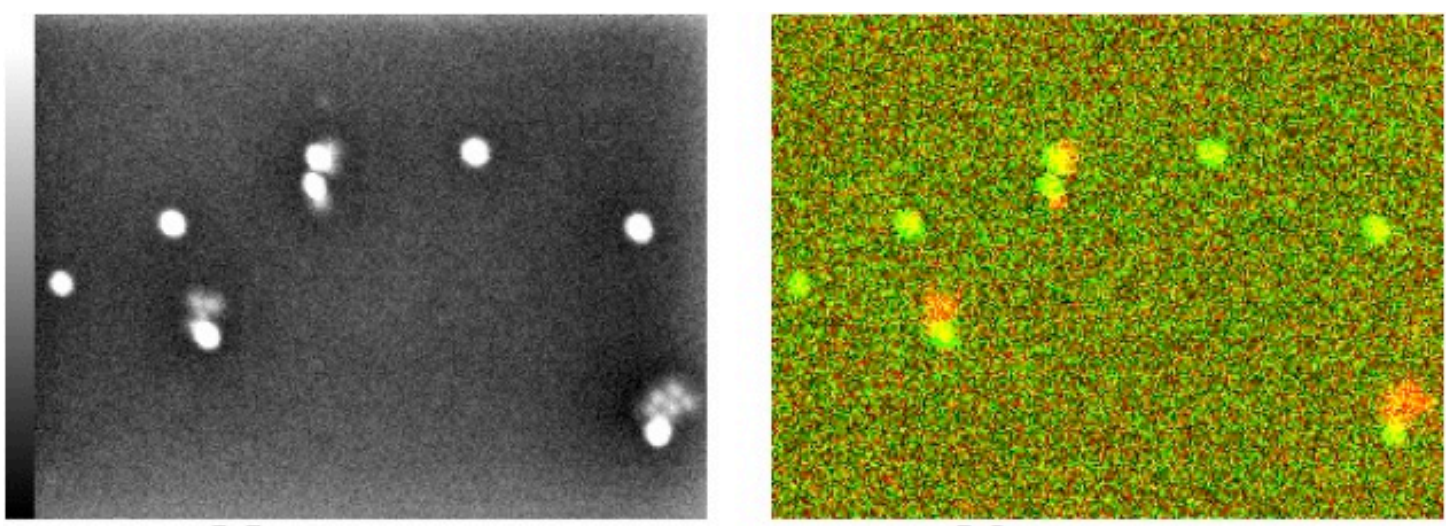

$2.0 \mu \mathrm{m}$

BF $2.0 \mu \mathrm{m}$
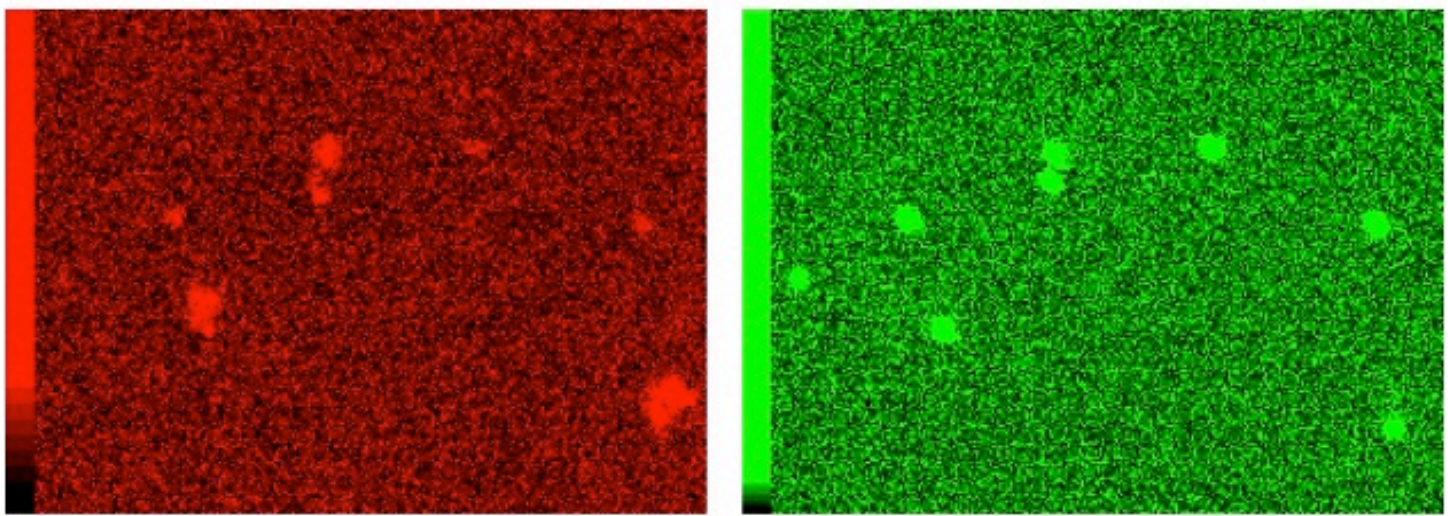

$2.0 \mu \mathrm{m}$

O K

$2.0 \mu \mathrm{m}$

Au M

Figure 4.12: EDS mapping of MG75 showing oxygen signal in the same areas as gold spheres. Top left: Backscatter image. Bottom left: Oxygen elemental map.

Bottom right: Gold elemental map. Top Right: Overlay of oxygen and gold elemental maps.

\subsubsection{Summary}

UV-Vis absorption spectroscopy showed that all samples exhibited two peaks except MG98-1, which exhibited only one peak at lower wavelength with greater 
intensity. This lower wavelength peak has increased intensity and a significant tail, and so it is possible it is masking a less intense, higher wavelength absorption. The absorption peaks observed at lower wavelengths occurring at approximately $550 \mathrm{~nm}$ were determined to be due to spherical gold nanoparticles also present in the sample. However, the minor second peaks observed in the spectra of all samples except MG98-1 are indicative of core-shell particles. SEM of samples prepared at $98{ }^{\circ} \mathrm{C}$, showed that all three samples produced core-shell particles. However, the samples had very few core-shell particles and many areas of gold aggregated together with silica spheres. The core-shell particles were found to be quite large, approximately $375 \mathrm{~nm}$ in diameter. This led to weak oxygen signals in the EDS analysis but some oxygen was detected leading to the conclusion that core-shell particles with thick shells were successfully formed. SEM of sample MG75 showed the formation of gold platelets in the sample, along with some areas of gold aggregated together with silica spheres and very few core-shell-like particles, also approximately $375 \mathrm{~nm}$ in diameter. These core-shell-like particles were analysed using EDS to show the presence of oxygen, confirming the core-shell structure.

The low wavelength peaks in the UV-Vis absorption spectra were determined to be due to spherical gold nanoparticles present in the sample. However, if this absorption was solely due to discrete spherical gold nanoparticles, these particles would be quite large, greater than $50 \mathrm{~nm}$ in diameter (see Figure 1.7), which would be seen in SEM images; spherical gold nanoparticles of this diameter are not seen in the SEM images. This indicates that the SPR absorption of spherical gold nanoparticles in these samples is shifted. As the wavelength of SPR absorption is dependent on the surrounding medium, this shift is considered to be caused by the higher refractive index of silica. It could be argued that this lower wavelength absorption is actually due to the core-shell particles in the sample. However, in order to exhibit SPR absorption of such a low wavelength the shells of these particles would need to be considerably thick. This thickness would induce a shoulder peak on this major peak, as shown in Figure 1.10. As this shoulder peak is not observed in these spectra it is determined that this is not the case. These lower wavelength peaks were also observed to exhibit significant tails. This also supports the notion that these peaks are caused by spherical gold nanoparticles, as aggregation of gold nanoparticles can induce tailing of the SPR absorption peaks, as seen in Figure 5.7. 


\subsection{In-situ Seeding on MPTMS Capped Silica with Hydroxylamine}

The MTPMS capping layer had been shown to effectively reduce $\mathrm{AuCl}_{4}{ }^{-}$to form gold shells. Here the use of MPTMS as a reductant for the initial in-situ seeding step is investigated with the use of hydroxylamine as an external reductant. As the reduction of $\mathrm{AuCl}_{4}{ }^{-}$by MPTMS was previously been investigated at $75^{\circ} \mathrm{C}$ and $98{ }^{\circ} \mathrm{C}$, the use of an additional, external reducing agent allows for the reaction temperature to be lowered. Hydroxylamine is a weak reductant and will only reduce $\mathrm{AuCl}_{4}{ }^{-}$onto a surface already decorated with gold; hence its use will also allow for more control over the growth of the gold shell.

Samples were prepared by adding MPTMS capped silica and $\mathrm{AuCl}_{4}{ }^{-}$to ethanol and heating at $50{ }^{\circ} \mathrm{C}$ followed by the addition of hydroxylamine solution either in sequential additions or in one addition. One sample also had subsequent additions of extra $\mathrm{AuCl}_{4}{ }^{-}$solution. See Section 2.4.5 In-situ Seeding on MPTMS Capped Silica Spheres with Hydroxylamine and Table 4.5 for full experimental details.

Table 4.5: Samples prepared via in-situ seeding on MPTMS capped silica spheres.

\begin{tabular}{|c|c|c|c|c|c|}
\hline $\begin{array}{c}\text { Sample } \\
\text { name }\end{array}$ & $\begin{array}{c}\text { Volume of } \\
\text { M-Silica } \\
(m L)\end{array}$ & 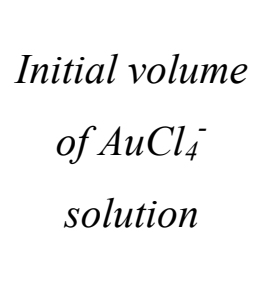 & $\begin{array}{l}\text { Volume of } \\
\text { ethanol } \\
(m L)\end{array}$ & $\begin{array}{c}\text { Volume of } \\
\text { hydroxylamine } \\
\text { solution }(\mathrm{mL})\end{array}$ & $\begin{array}{c}\text { Volume of } \\
\text { additional } \\
\mathrm{AuCl}_{4}^{-} \text {solution } \\
(\mu L)\end{array}$ \\
\hline MG & 2.5 & $1.22 \mathrm{~mL}$ & 6.28 & 2.0 & 0.0 \\
\hline $\mathrm{MGa}$ & 2.5 & $1.22 \mathrm{~mL}$ & 6.28 & $4 \times 0.5$ & 0.0 \\
\hline MGb & 1.0 & $50 \mu \mathrm{L}$ & 9.0 & 0.209 & $2 \times 50$ \\
\hline
\end{tabular}


MG and MGa samples were both left for 5 days to allow for the reduction of gold seeds by the MPTMS capping layer before addition of hydroxylamine solution to grow the gold shell. The hydroxylamine solution was added to MGa over 2 days, with two $0.5 \mathrm{~mL}$ additions per day. Both $\mathrm{MG}$ and $\mathrm{MGa}$ were kept at $50{ }^{\circ} \mathrm{C}$ for a total of 8 days. MGb was left to reduce gold seeds only overnight before the addition of hydroxylamine solution, and subsequent additions of $\mathrm{AuCl}_{4}{ }^{-}$were performed when all yellow colour was lost from the sample or after 6 hours (whichever occurred first). MGb was kept at $50{ }^{\circ} \mathrm{C}$ for a total of only 3 days.

During the synthesis, these samples changed colour from translucent yellow to opaque brown and specks of bulk gold settled out in MG and MGa. The formation of bulk gold in these samples indicated that the concentration of gold was much too high, hence $\mathrm{MGb}$ was synthesised with much less $\mathrm{AuCl}_{4}{ }^{-}$and no bulk gold was seen in the sample. The yellow colour of the initial $\mathrm{AuCl}_{4}{ }^{-}$was lost after the addition of hydroxylamine solution and the sample became white, however the colour remained after further additions of $\mathrm{AuCl}_{4}{ }^{-}$, with the sample developing a slightly orange colour by the end of the synthesis. The final colours of the samples are shown in Figure 4.13. It was observed that samples MG and MGb appeared different colours in reflected and transmitted light. MG appeared dark orange in reflected light and purple in transmitted light, MGb appeared dull brown in reflected light and slightly blue in transmitted light. All three samples were analysed using UV-Vis absorption spectroscopy and SEM, MGa and MGb were also analysed using XRD and MGb was finally analysed using TEM.

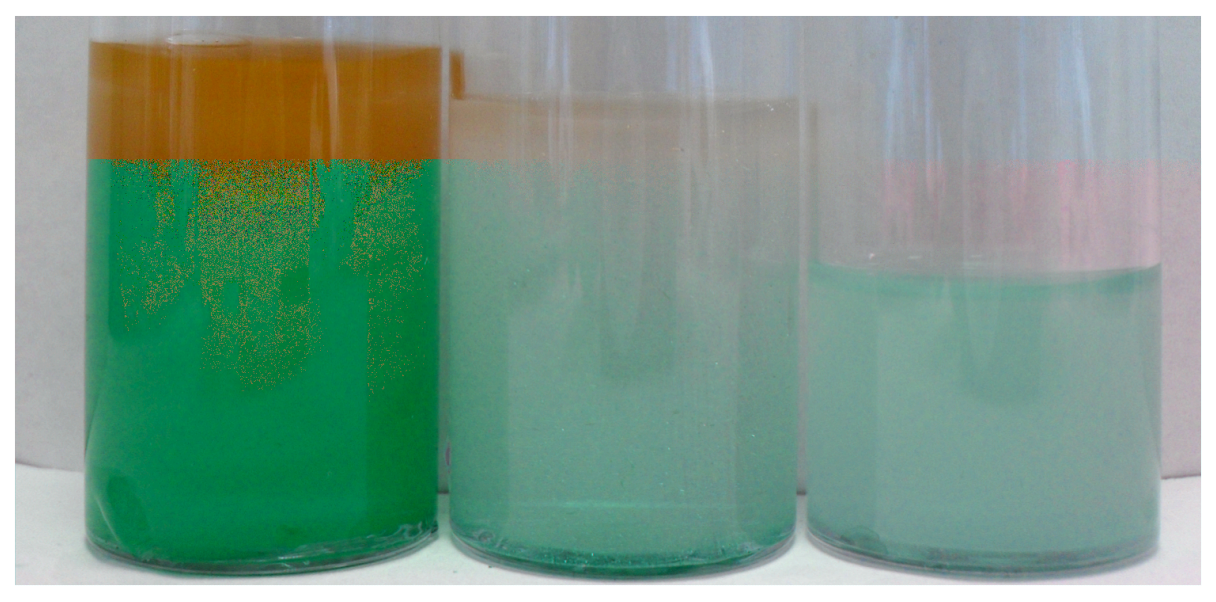

Figure 4.13: Photograph of samples $M G, M G a$ and $M G b$ (left to right). 


\subsubsection{UV-Vis Absorption Spectroscopy}

As seen in Figure 4.14, the UV-Vis absorption spectra of these samples showed no peaks in the visible region. MG showed a small broad absorption at approximately $520-700 \mathrm{~nm}$, this suggested the presence of bulk gold particles in the sample of many different sizes and shapes.

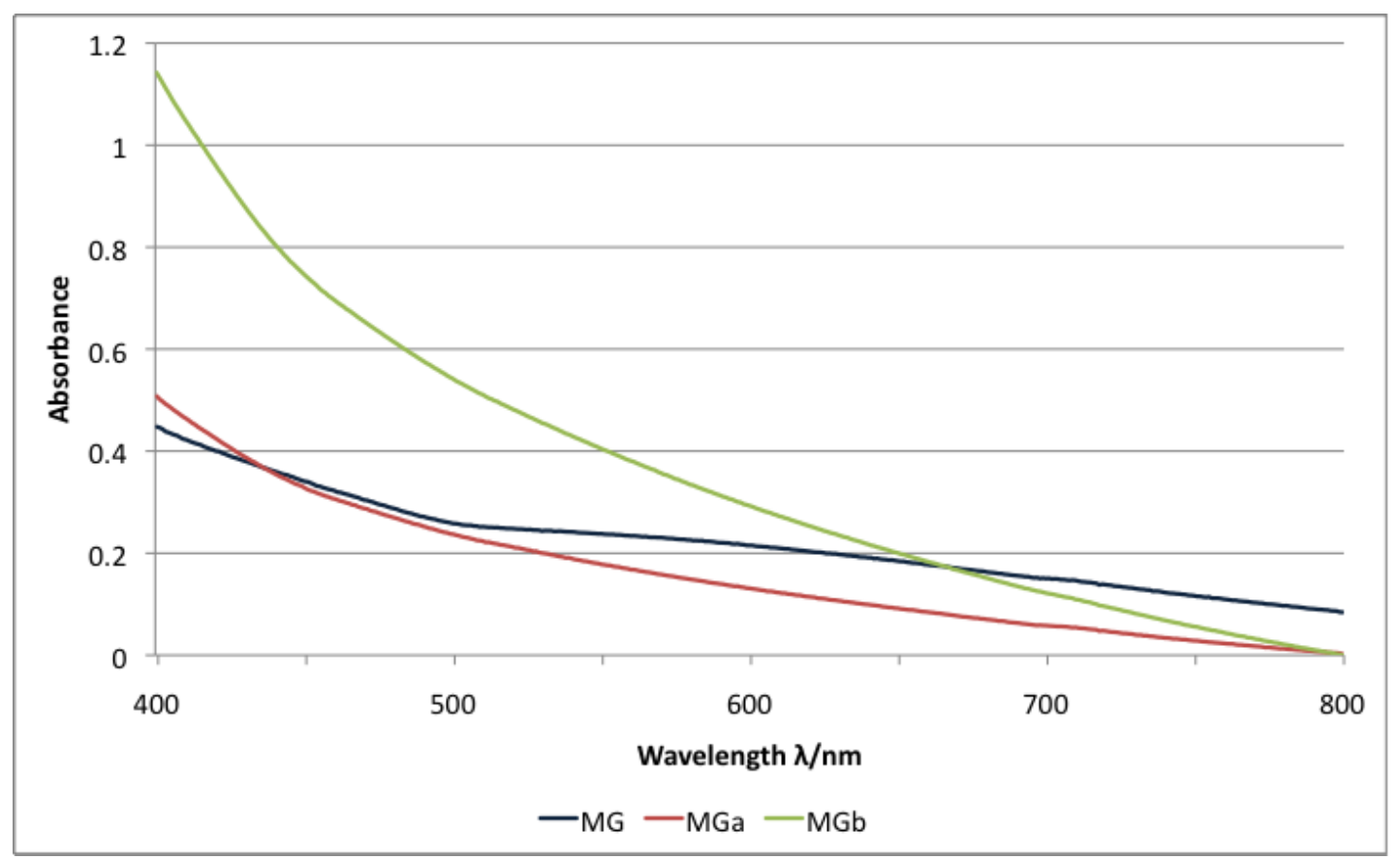

Figure 4.14: UV-Vis absorption spectra of $M G, M G a$ and $M G b$, prepared via in-situ seeding on MPTMS capped silica with hydroxylamine. Samples were not of equal concentrations.

\subsubsection{SEM}

All samples were coated with $8 \mathrm{~nm}$ of carbon and imaged on the JEOL-6500F instrument. SEM imaging of MG and MGa showed the presence of many large gold microplatelets of varying size. This supports the observation of specks of bulk gold in the samples. Some areas of small gold nanoparticles were found on the silica spheres, however no core-shell particles were found in the samples. No SPR absorption peaks 
were seen in the UV-Vis absorption spectra of these samples, indicating that the amount of gold nanoparticles in the samples is very low. Representative SEM images of samples MG and MGa are shown in Figure 4.15.
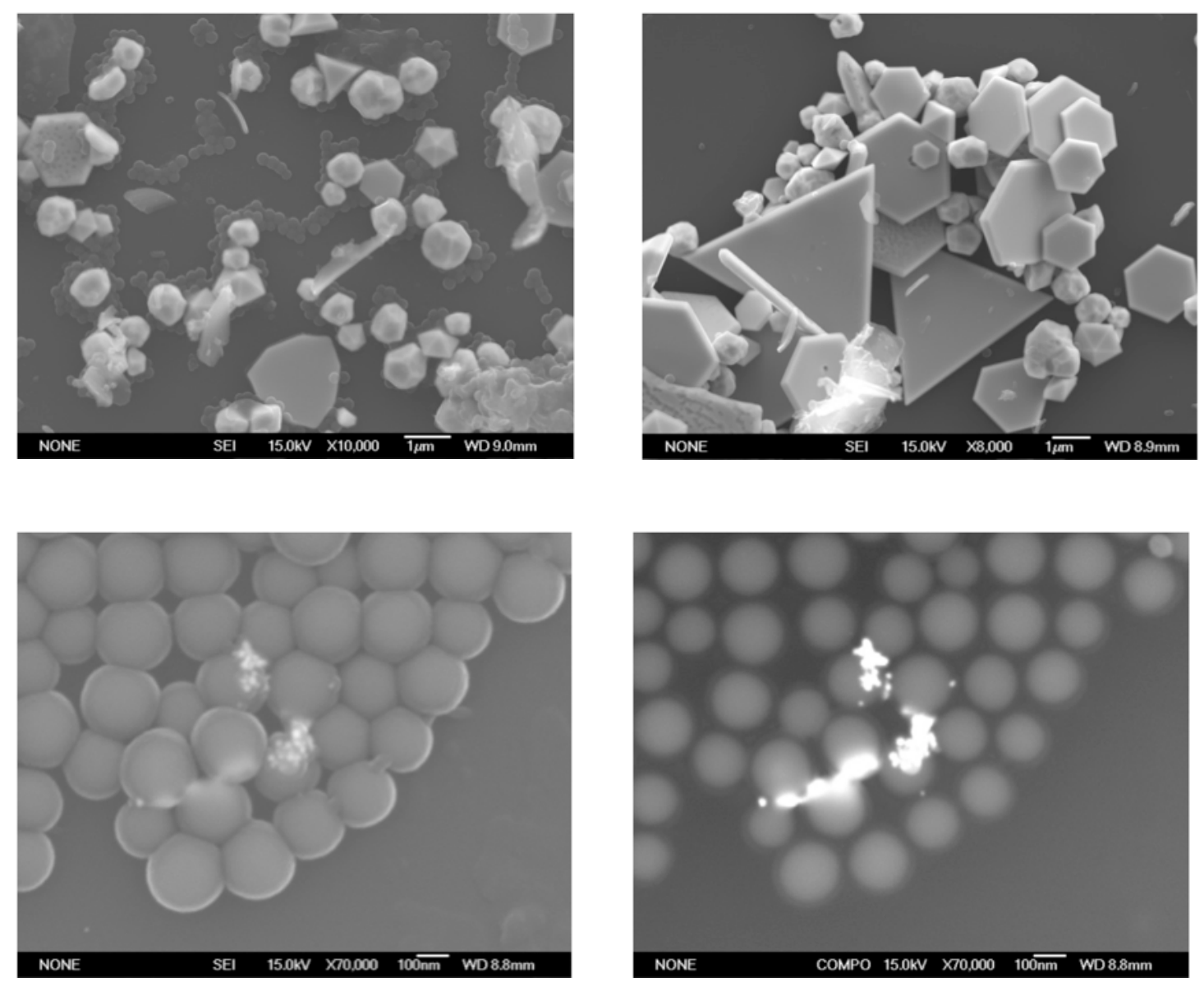

Figure 4.15: SEM images of MG (top) in SEI mode and MGa (bottom) in both SEI (left) and backscatter mode (right).

As seen in Figure 4.16, SEM imaging of MGb revealed that there was an abundance of gold surrounding the MPTMS capped silica spheres in the sample. This was an important result as it indicates that the gold had been successfully adsorbed into the MPTMS capping layer. However, this gold did not appear crystalline and it was suspected that the gold present was $\mathrm{AuCl}_{4}{ }^{-}$not fully reduced to gold metal. This agrees with the UV-Vis absorption spectrum, which did not show the presence of an SPR absorption band, as the gold in the sample remained $\mathrm{AuCl}_{4}^{-}$, which is not SPR active. Upon closer inspection, it was determined that within these areas of unreduced 
gold, there were some small gold nanoparticles. This indicated that some reduction of $\mathrm{AuCl}_{4}{ }^{-}$to $\mathrm{Au}^{0}$ did occur, but the majority of the gold remained oxidised. It is assumed that the gold nanoparticles found in the sample did not exhibit an observable SPR absorption band in the UV-Vis absorption spectrum, as there were very few of them in the sample.
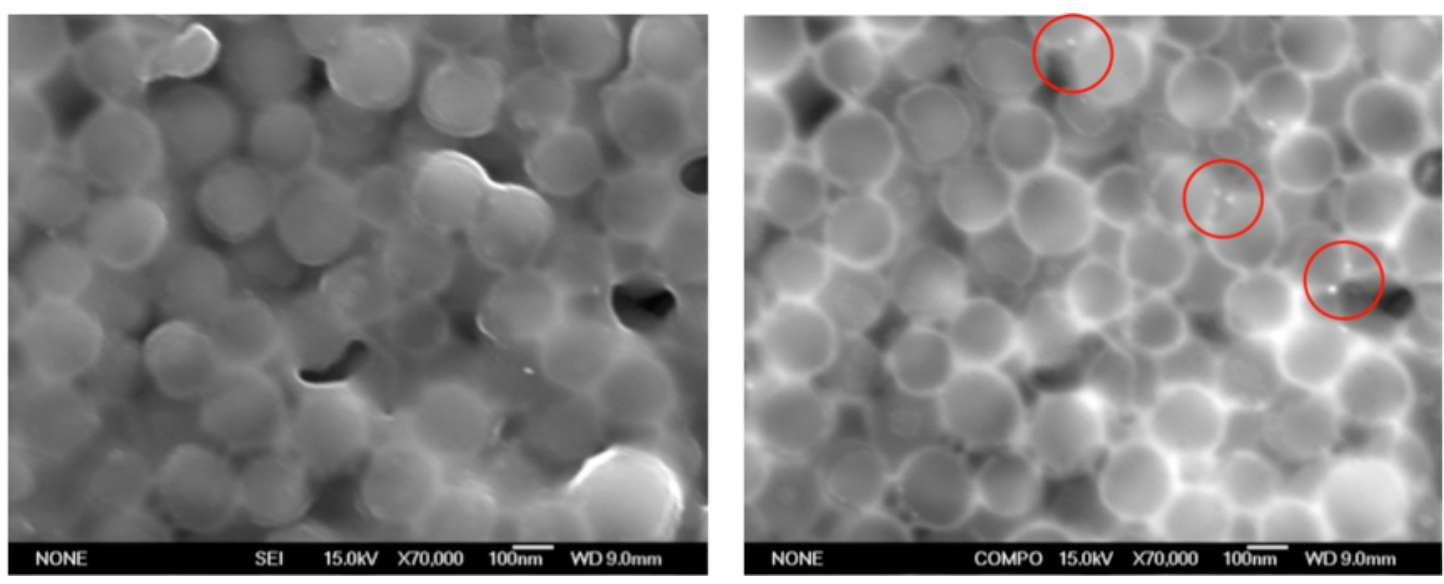

Figure 4.16: SEM images of MGb in both SEI (left) and backscatter (right) modes. Red circles in the backscatter image show some areas where small gold nanoparticles have formed. 


\subsubsection{XRD}

XRD analysis of MGa confirmed the presence of crystalline gold metal. This is expected as large flecks of gold were observed in the sample.

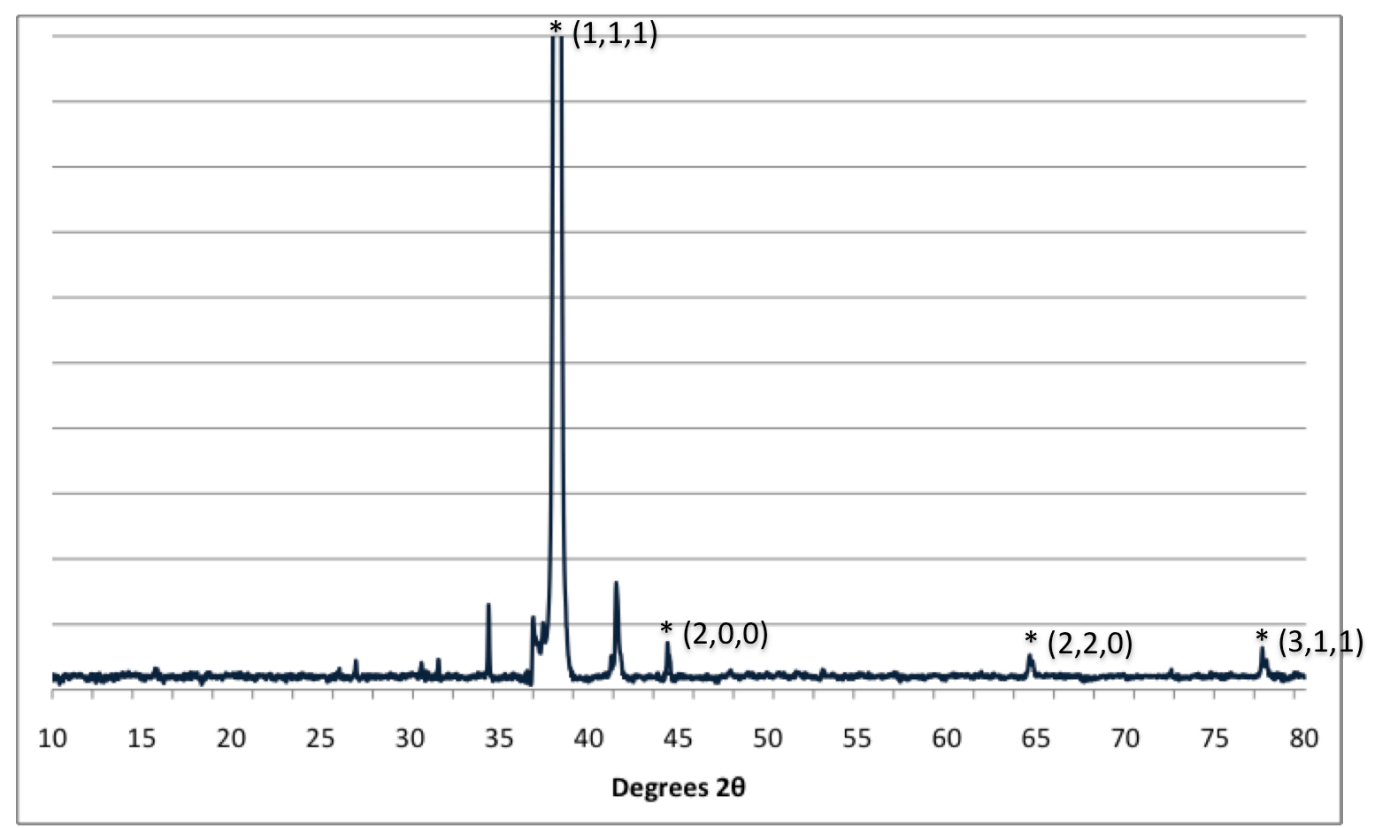

Figure 4.17: XRD spectrum of sample MGa showing peaks due to crystal planes of gold metal.

$\mathrm{XRD}$ analysis of MGb also showed the presence of crystalline gold metal. This supports the conclusions drawn from the SEM analysis, that although there was gold in the sample that was not fully reduced and remained as $\mathrm{AuCl}_{4}^{-}$, the hydroxylamine and MPTMS capping layers had sufficient reducing power to reduce some of the $\mathrm{Au}^{3+}$ to $\mathrm{Au}^{0}$ in the sample to form a few gold nanoparticles. 


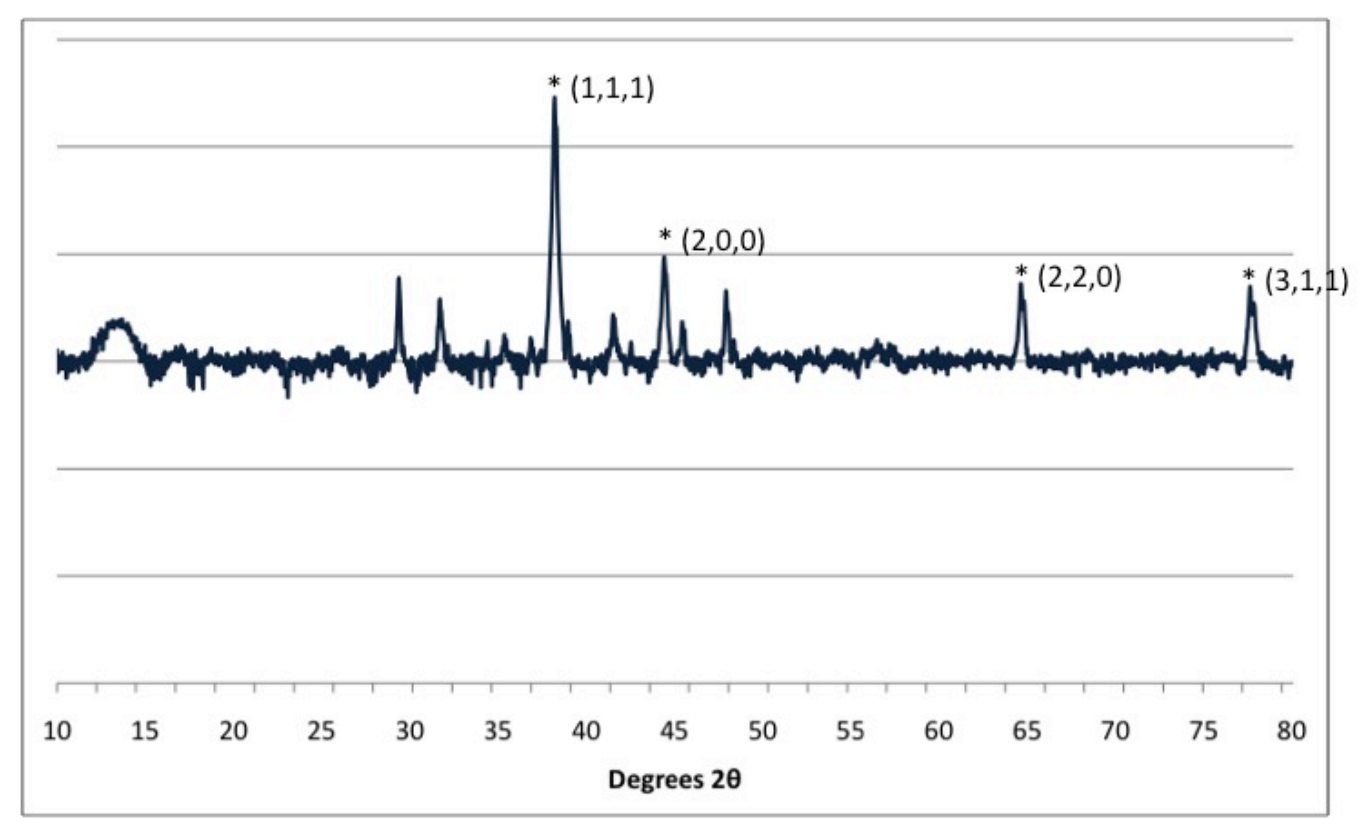

Figure 4.18: XRD spectrum of sample MGb showing peaks due to crystal planes of gold metal.

\subsubsection{TEM}

TEM characterisation of MGb revealed the presence of some small gold nanoparticles in the sample, seen in Figure 4.19, as well as some larger areas of gold associated with the silica spheres, but these larger areas did not form core-shell particles, as seen in Figure 4.20. The STEM EDS images of these larger gold areas showed a non-uniform silicon map in the area of the gold. This indicates that the large area of gold is not a shell on the surface of one silica sphere, rather it is a large clump of gold on a silica sphere aggregate. This confirms that although a lot of gold was adsorbed into the MPTMS capping layer, as seen in SEM images, this gold was not always reduced, and where it was, there were insufficient gold nanoparticles to form continuous gold shells. 


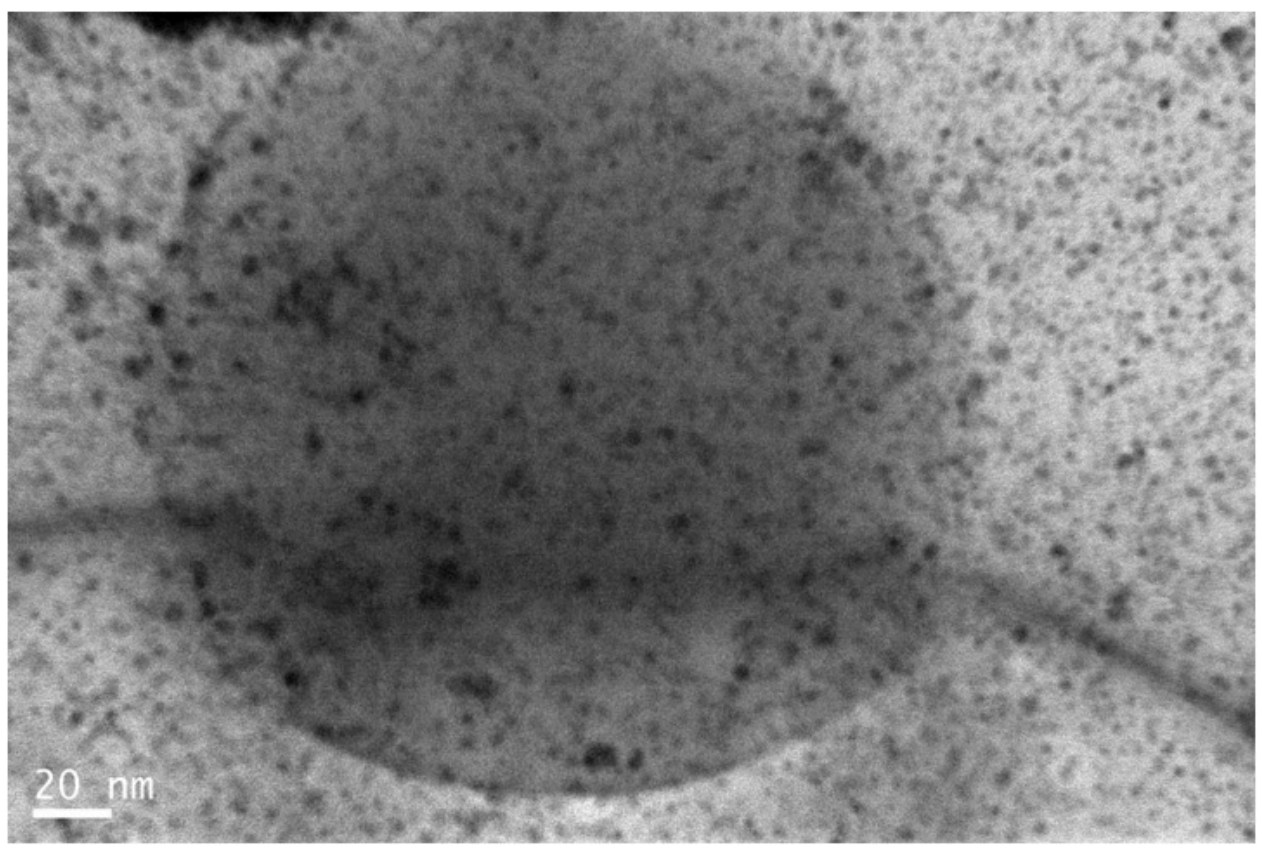

Figure 4.19: TEM image of MGb showing small gold nanoparticles (darker spots) and a large silica sphere. 
$2.0 \mu \mathrm{m}$

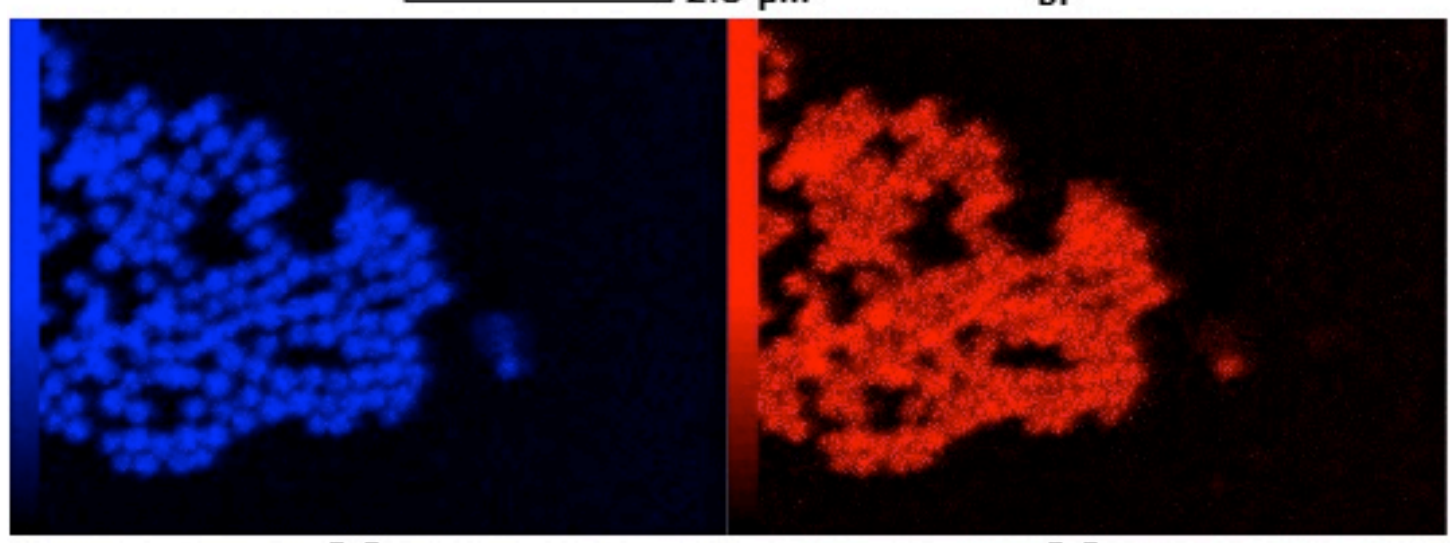

$2.0 \mu \mathrm{m}$

Si K

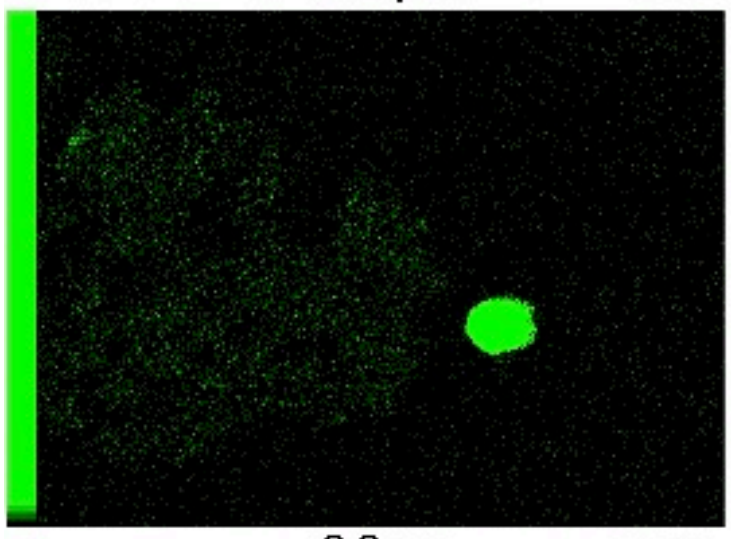

Au M

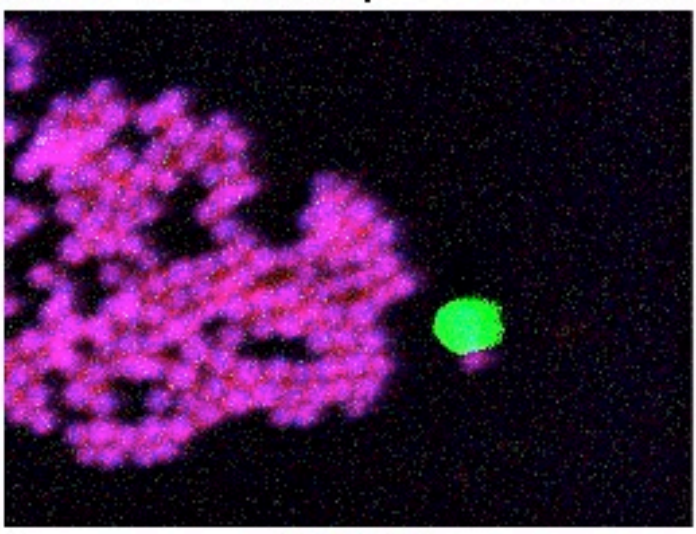

$2.0 \mu \mathrm{m}$

Figure 4.20: STEM EDS images of MGb showing large area of gold associated with silica spheres. Top: STEM image. Middle left: Silicon map. Middle right: Oxygen map. Bottom left: Gold map. Bottom right: Overlap of silicon, oxygen and gold elemental maps. 


\subsubsection{Summary}

MG and MGa were determined to be unsuccessful in synthesising core-shell particles, instead forming large gold platelets in the sample, not associated with the silica spheres. MGb was found to have gold successfully adsorbed into the MPTMS capping layer. However, this gold was not always fully reduced so no core-shell particles were found in the sample. This led to the investigation of adaptations to this synthetic method, using more reducing agents in an effort to successfully reduce the gold adsorbed onto the silica and successfully form core-shell particles.

\subsection{Adaptations of MGb Synthesis}

The sample MGb was determined to have successfully adsorbed gold into the MPTMS capping layer of the silica spheres. However, it was found that this gold was not fully reduced, and hence crystalline gold shells were not formed. Here, changes to reaction parameters were investigated in an attempt to fully reduce the gold in the system and form gold shells. The gold was successfully adsorbed into the capping layer in the original MGb synthesis but as this is a vital step, the time allowed for this process was increased in all further syntheses to ensure maximum adsorption occurred. Other reaction parameters that were altered included both initial and final volumes of $\mathrm{AuCl}_{4}{ }^{-}$solution, solvent, volume of silica and volume of hydroxylamine solution used, as well as investigation into the use of sodium borohydride.

Samples were prepared by adding $\mathrm{AuCl}_{4}{ }^{-}$to MPTMS capped silica in solvent. These samples were then kept at $50{ }^{\circ} \mathrm{C}$ for 3 days before further reagents were added (60 hours more than original MGb synthesis) and until at least 6 hours after the final addition of $\mathrm{AuCl}_{4}{ }^{-}$. After the initial yellow colour was lost or at least 6 hours, further additions of $\mathrm{AuCl}_{4}{ }^{-}$or hydroxylamine solution were added to the samples, with at least 6 hours between additions of $\mathrm{AuCl}_{4}^{-}$. Samples were then returned to room temperature and one sample had $\mathrm{NaBH}_{4}$ added. Full experimental details can be found in Section 2.4.5 In-situ Seeding on MPTMS Capped Silica Spheres with Hydroxylamine, and changes to the original MGb synthesis are summarised in Table 4.6. 
MGb-a had water as the solvent in place of ethanol in the original MGb synthesis. Before the addition of hydroxylamine solution, the sample had already lost the initial yellow colour and appeared slightly opaque white with a tinge of orange, and some orange material had plated the sides of the vial. The original MGb sample had not lost the yellow colour at this stage, which implies that the water solvent promotes the reduction of $\mathrm{AuCl}_{4}{ }^{-}$(source of yellow colour). By the end of the synthesis MGb-a had developed a strong orange colour.

MGb-b was left at $50{ }^{\circ} \mathrm{C}$ for 3 days before the hydroxylamine solution was added to the sample. The sample was left for longer to allow more time for the gold to be absorbed into the MPTMS capping layer and be reduced. This sample was observed to react in a similar way to the original MGb sample, but no orange colour was developed and the sample remained white.

MGb-c had more $\mathrm{AuCl}_{4}{ }^{-}$in the initial sample than the original $\mathrm{MGb}$ sample. This sample lost all initial yellow colour after the addition of hydroxylamine solution. After the first extra $\mathrm{AuCl}_{4}{ }^{-}$addition the sample had developed a slight orange colour that persisted throughout the rest of the synthesis, similar to the reaction of the original MGb sample.

MGb-d had extra $\mathrm{AuCl}_{4}{ }^{-}$added in the sequential addition steps of the original MGb synthesis. After the addition of hydroxylamine solution, the sample lost all initial yellow colour and lost the yellow colour after each further addition of $\mathrm{AuCl}_{4}^{-}$, leaving a colourless solution with white precipitate, no other colour was formed in the synthesis.

MGb-e had $\mathrm{NaBH}_{4}$ added at the very end of the synthesis in an effort to effectively reduce all the $\mathrm{AuCl}_{4}{ }^{-}$left in the sample. Until the point where $\mathrm{NaBH}_{4}$ was added this sample reacted the same as the original MGb sample and a slight orange colour was developed. After the addition of $\mathrm{NaBH}_{4}$ the orange colour became much more intense and slightly browner, and the sample was observed to appear purple in transmitted light.

MGb-f had less silica in the sample. The sample appeared less opaque than the original MGb sample but mostly appeared to react the same. 
MGb-g had both more $\mathrm{AuCl}_{4}{ }^{-}$added to the sample (as did MGb-c) as well as more hydroxylamine solution added. This sample appeared to react in a similar way to the original MGb sample; the colour of the final sample was similar to that of MGb.

The final colour of all samples is shown in Figure 4.21. It was observed that samples MGb-a, -c, -e, -f, -g appeared different colours in reflected and transmitted light. The samples appeared a shade of brown/orange in reflected light and more blue/purple in transmitted light. Samples MGb-b and MGb-d did not have this property.

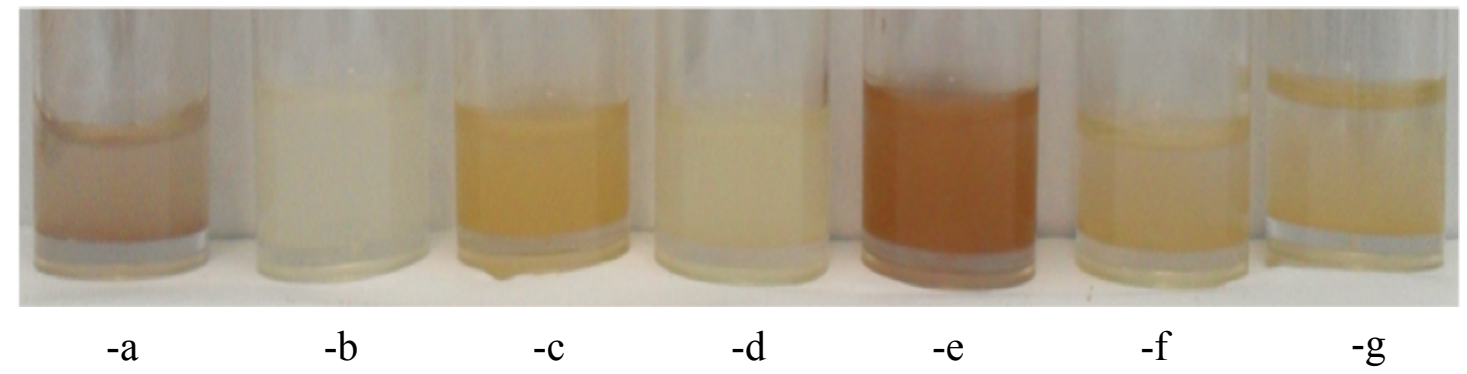

Figure 4.21: Photograph of samples MGb-a, -b, -c, -d, -e, -f, -g (left to right). 
Table 4.6: Adaptations of MGb synthesis, altering time kept at $50{ }^{\circ} \mathrm{C}$ in addition to solvent, volume of silica, initial and added volumes of $\mathrm{AuCl}_{4}^{-}$, volume of hydroxylamine solution or volume of $\mathrm{NaBH}_{4}$.

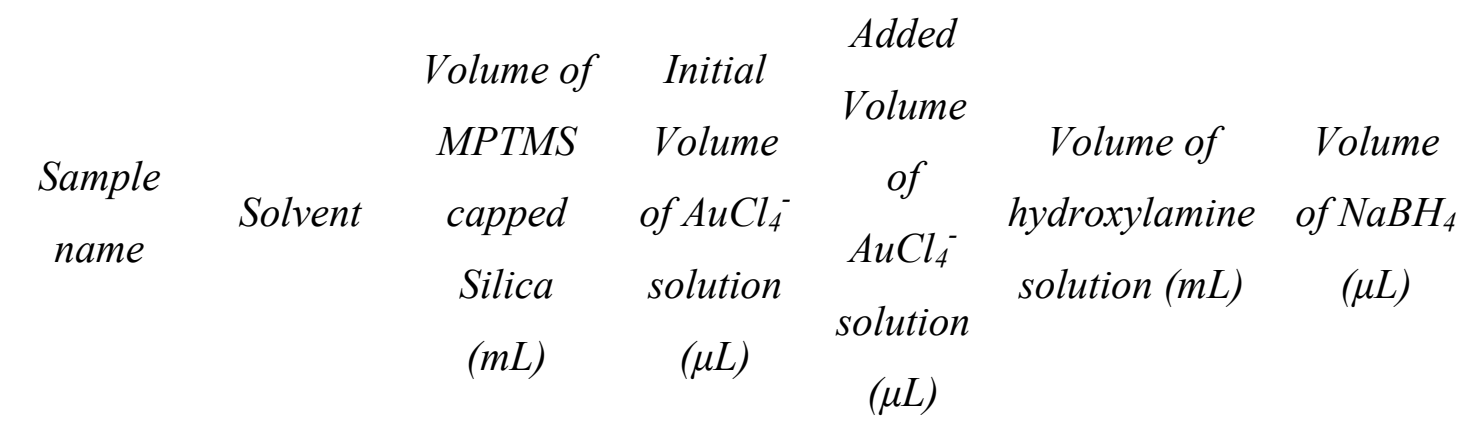

\begin{tabular}{|c|c|c|c|c|c|c|}
\hline $\mathrm{MGb}$ & $\begin{array}{l}9.0 \mathrm{~mL} \\
\text { ethanol }\end{array}$ & 1.0 & 50 & $2 \times 50$ & 0.209 & 0 \\
\hline MGb-a & $\begin{array}{l}9.0 \mathrm{~mL} \\
\text { water }\end{array}$ & 1.0 & 50 & $2 \times 50$ & 0.209 & 0 \\
\hline MGb-b & $\begin{array}{l}9.0 \mathrm{~mL} \\
\text { ethanol }\end{array}$ & 1.0 & 50 & $2 \times 50$ & 0.209 & 0 \\
\hline MGb-c & $\begin{array}{l}9.0 \mathrm{~mL} \\
\text { ethanol }\end{array}$ & 1.0 & 75 & $2 \times 50$ & 0.209 & 0 \\
\hline MGb-d & $\begin{array}{l}9.0 \mathrm{~mL} \\
\text { ethanol }\end{array}$ & 1.0 & 50 & $\begin{array}{c}2 \times 50+ \\
25\end{array}$ & 0.209 & 0 \\
\hline MGb-e & $\begin{array}{l}9.0 \mathrm{~mL} \\
\text { ethanol }\end{array}$ & 1.0 & 50 & $2 \times 50$ & 0.209 & 30 \\
\hline MGb-f & $\begin{array}{l}9.5 \mathrm{~mL} \\
\text { ethanol }\end{array}$ & 0.5 & 50 & $2 \times 50$ & 0.209 & 0 \\
\hline MGb-g & $\begin{array}{l}9.0 \mathrm{~mL} \\
\text { ethanol }\end{array}$ & 1.0 & 75 & $2 \times 50$ & $0.209+0.1$ & 0 \\
\hline
\end{tabular}




\subsubsection{UV-Vis Absorption Spectroscopy}

All samples were investigated using UV-Vis absorption spectroscopy. The absorption spectra showed all samples had a small peak at approximately $710 \mathrm{~nm}$ indicative of core-shell particles (see Figure 4.22), while MGb-e and MGb-f also had a slightly more intense peak at $525 \mathrm{~nm}$ characteristic of spherical gold nanoparticles (see Figure 4.23).

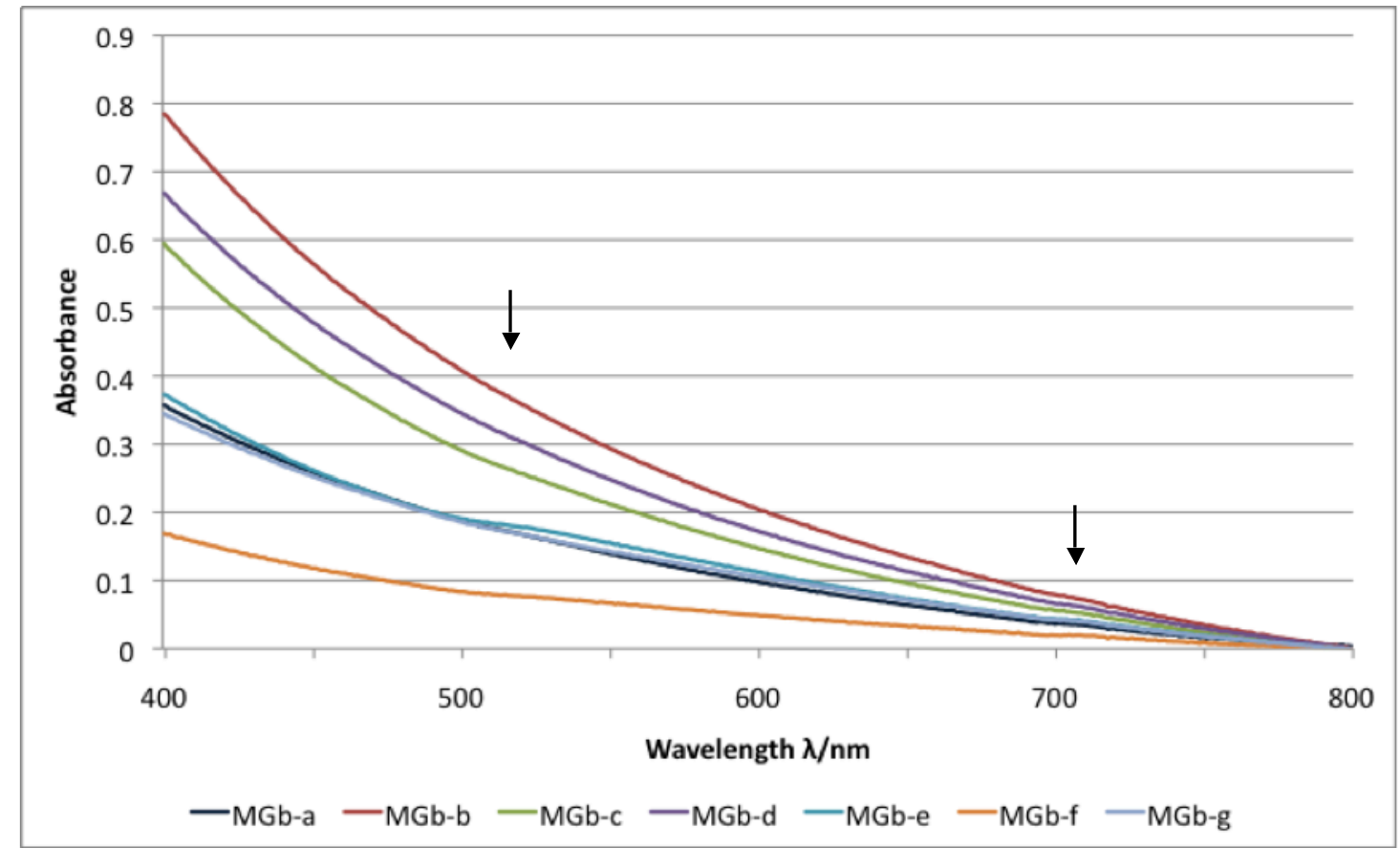

Figure 4.22: UV-Vis absorption spectra of samples prepared via the adaptation of MGb synthesis, all showing peaks at approximately $710 \mathrm{~nm}$ (samples were not of equal concentrations). 


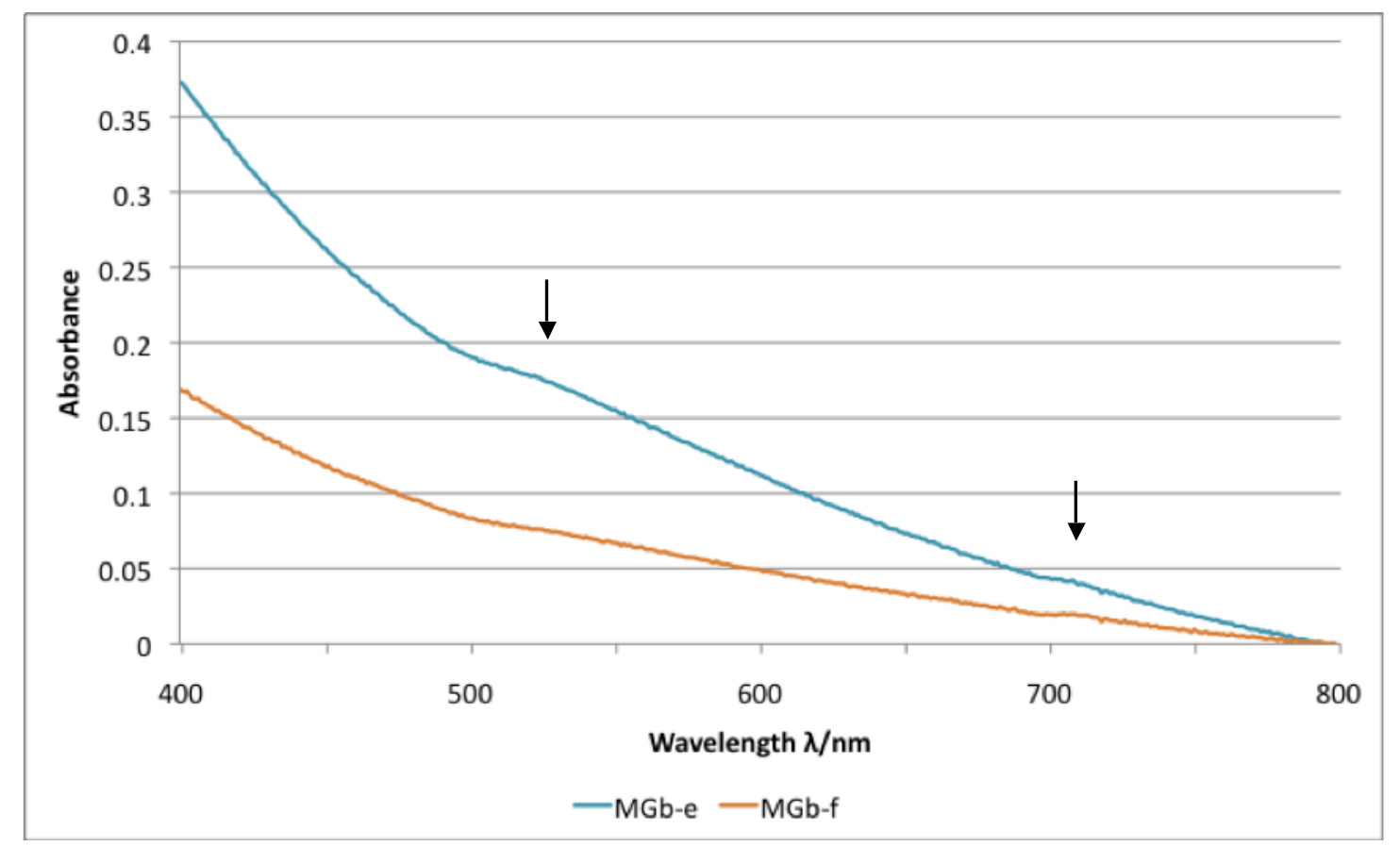

Figure 4.23: UV-Vis absorption spectra of $M G b$-e and MGb-f prepared via adaptations of MGb synthesis showing peaks at approximately $525 \mathrm{~nm}$, deemed to be due to the presence of spherical gold nanoparticles in the samples (samples were not of equal concentrations).

\subsubsection{SEM}

Samples were analysed on JEOL-JSM-6610LA instrument and were coated with $10 \AA$ of platinum. The platinum coat caused interference with gold signals in backscatter mode and in EDS mapping so the signal range was manipulated in EDS software to eliminate any signal overlap.

Some triangular and hexagonal platelets of high-density material were found in samples MGb-b, MGb-c, MGb-e and MGb-g, shown in Figure 4.24. These were determined to be gold as gold can take on hexagonal close packed structure causing triangular and hexagonal shaped crystals and is of high-density. The formation of these platelets indicated an excess of gold in the reaction. 


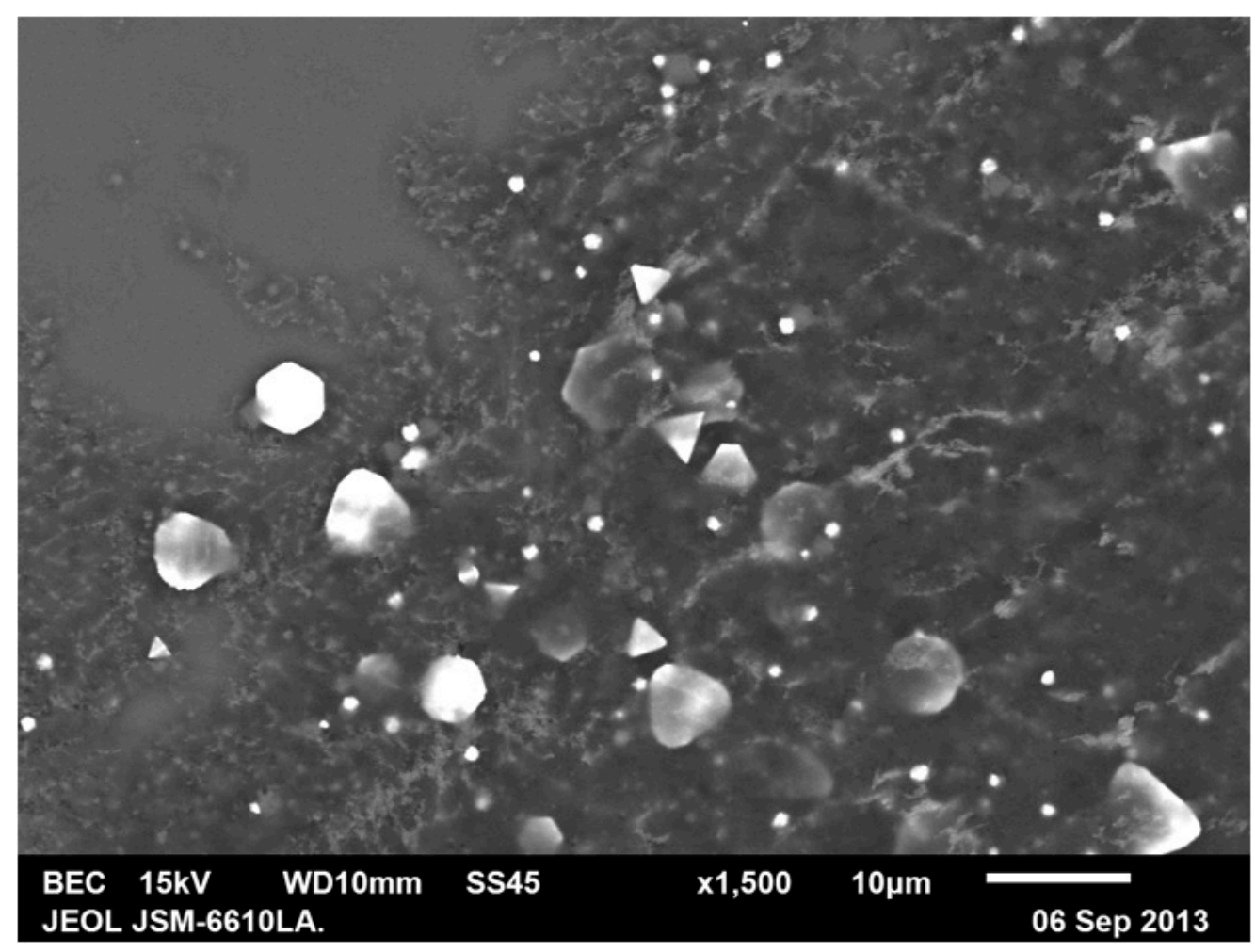

Figure 4.24: SEM image of MGb-c in backscatter mode representative of samples $M G b-b, M G b-c, M G b-e$ and $M G b-g$, showing triangular and hexagonal platelets of high-density material.

Some spherical particles of high-density material of approximately the size of silica spheres, approximately $200 \mathrm{~nm}$ in diameter, were found in MGb-f and these were analysed using EDS mapping (see Figure 4.25). Silicon was not mapped in this analysis as the samples were drop cast onto a silicon wafer, which interferes with the silicon mapping. This confirmed that the high-density material was gold and that the shape was a shell over an oxygen-containing compound, deemed to be silica. This indicates that it is likely that the spherical particles found in other samples were also silica-gold core-shell particles.

Similar spherical particles of high-density material of approximately the size of silica spheres, approximately $200 \mathrm{~nm}$ in diameter, were found in MGb-a, MGb-d, MGb-e and MGb-g; this suggested that silica-gold core-shells had formed in these samples also. However EDS analysis was not carried out on these samples so it was 
not confirmed that this high-density material was gold or that the spherical shape was representative of a shell over silica.

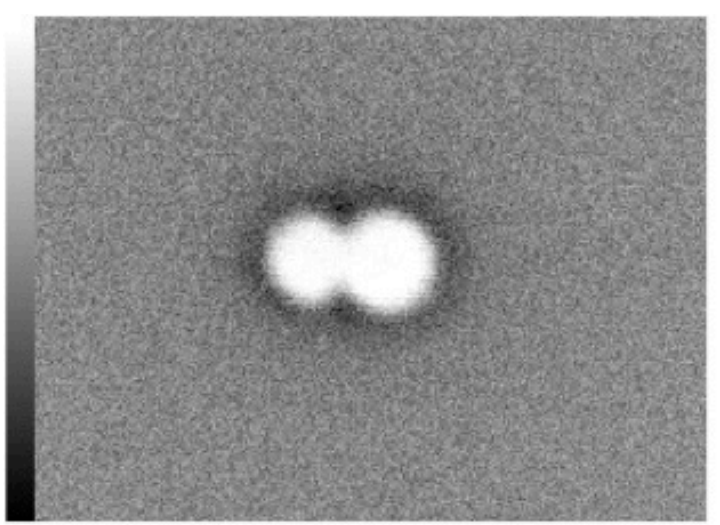

$0.5 \mu \mathrm{m}$

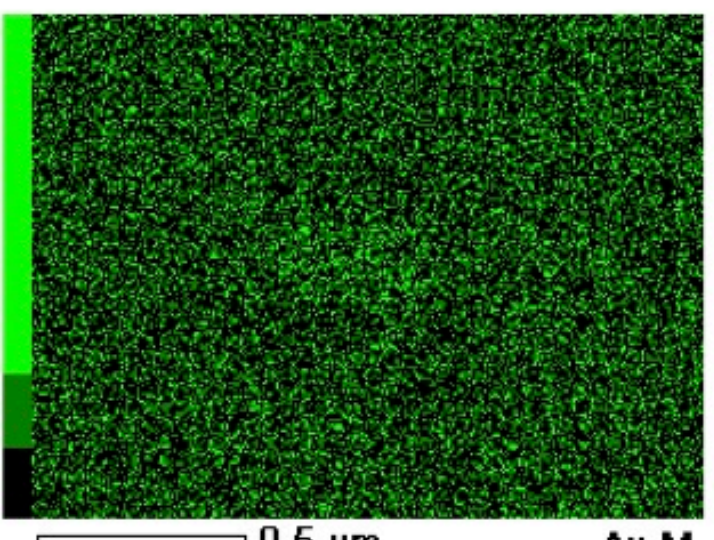

$0.5 \mu \mathrm{m}$
BF(frame1)

Au M

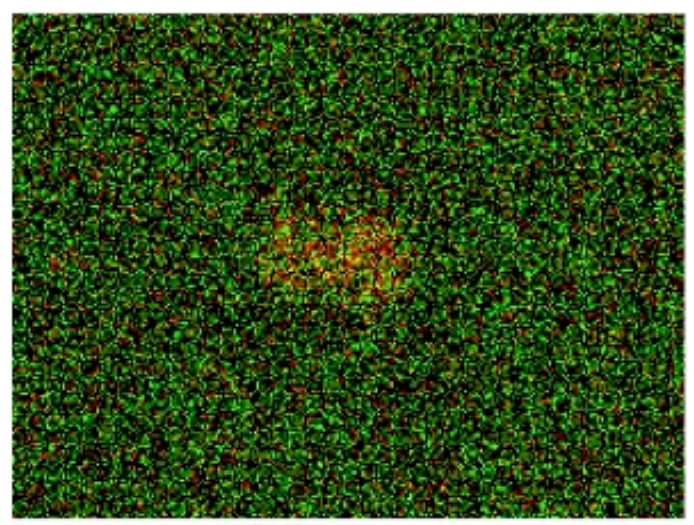

$0.5 \mu \mathrm{m}$

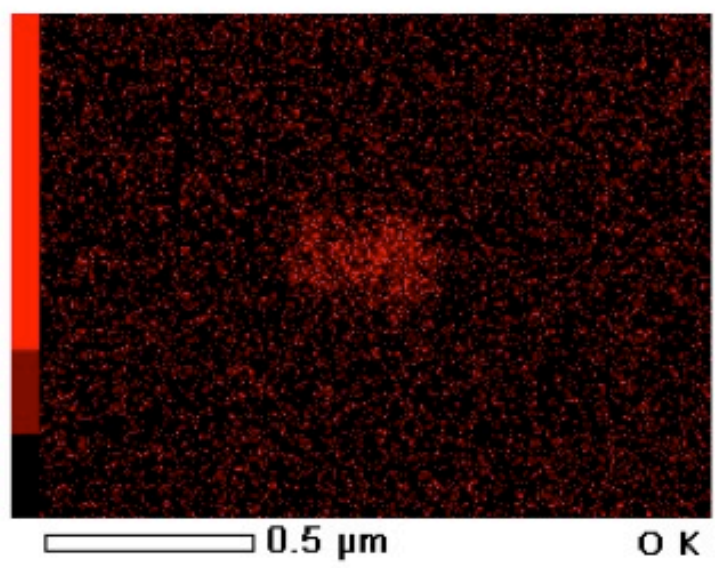

Figure 4.25: EDS mapping of MGb-f showing silica-gold core-shell particle.

Top left: Backscatter image. Bottom left: Gold map. Bottom right: Oxygen map.

Top right: Overlay of gold and oxygen elemental maps.

\subsubsection{Summary}

UV-Vis absorption spectra indicated that core-shell particles could be present in all samples due to all spectra showing small absorption peaks at approximately $700 \mathrm{~nm}$. It was also found that samples MGb-e and MGb-f likely contained spherical gold nanoparticles as their respective spectra also showed absorption peaks at approximately $525 \mathrm{~nm}$. SEM showed no evidence of core-shell particles in MGb-b 
and MGb-c, some evidence of potential core-shell particles in MGb-a, MGb-d, MGb-e and MGb-g and core-shells were found in MGb-f and confirmed with EDS. Some gold platelets were seen to have formed in samples MGb-b, MGb-c, MGb-e and MGb-g, indicating the samples contained a significant excess of gold.

Synthesis involving half the amount of silica in the original MGb synthesis was confirmed to have successfully formed core-shell particles and it was deemed likely that syntheses involving water as solvent, more additional gold, more initial gold and more hydroxylamine and the use of $\mathrm{NaBH}_{4}$, also successfully formed core-shell particles. Only syntheses with half the amount of silica or with $\mathrm{NaBH}_{4}$ yielded samples with absorption peaks at approximately $525 \mathrm{~nm}$ in their respective UV-Vis absorption spectra. Hence it was determined that other syntheses did not produce significant amounts of spherical gold nanoparticles.

\subsection{In-situ Seeding on MPTMS Capped Silica Spheres with $\mathrm{NaBH}_{4}$}

The use of sodium borohydride as an external reducing agent in the seeding step of previous syntheses using hydroxylamine was proven to be beneficial. The effect of sodium borohydride in these syntheses was investigated further by altering the volume of sodium borohydride used.

Samples were prepared by adding $50 \mu \mathrm{L}_{\text {of }} \mathrm{AuCl}_{4}{ }^{-}$to $1 \mathrm{~mL}$ of MPTMS capped silica in $9 \mathrm{~mL}$ of ethanol. After 2 hours at room temperature $\mathrm{NaBH}_{4}$ was added to these samples and left at room temperature overnight. Subsequently, $0.209 \mathrm{~mL}$ of hydroxylamine solution was added to each solution and the solutions were left at $50{ }^{\circ} \mathrm{C}$ until the yellow colour was lost. Two further additions of $50 \mu \mathrm{L}$ of $\mathrm{AuCl}_{4}{ }^{-}$were then added to each sample and the samples were left at $50{ }^{\circ} \mathrm{C}$ after each addition until the yellow colour had been lost, or for 6 hours (whichever occurred first). The samples were left at $50{ }^{\circ} \mathrm{C}$ for a total of 3 days. See Section 2.4.6 In-situ Seeding on MPTMS Capped Silica Spheres with $\mathrm{NaBH}_{4}$ and Hydroxylamine and Table 4.7 for full experimental details. 
Table 4.7: Samples prepared via in-situ seeding on MPTMS capped silica spheres with $\mathrm{NaBH}_{4}$.

Sample name

MGN1

MGN2

MGN3
Volume of $\mathrm{NaBH}_{4}(\mu L)$

10

15

20
$\mathrm{NaBH}_{4}: \mathrm{AuCl}_{4}^{-}$mole ratio

$1: 2$

$2: 3$

$1: 1$

Upon addition of $\mathrm{NaBH}_{4}$ to the samples a darker colour was seen in the colloid where the $\mathrm{NaBH}_{4}$ solution had flowed. This colouration disappeared upon mixing. After being left overnight some of the yellow colour had been lost and the samples appeared browner with some pink/purple precipitate. After addition of hydroxylamine solution the samples appeared opaque brown, with MGN3 having a slight pink colour to it, and MGN1 being the darkest of the three. These colours remained throughout the rest of the experiment. It was noted that the samples appeared different colours in reflected light and transmitted light. All samples appeared brownish orange in reflected light and purple in transmitted light.

All three samples were analysed using UV-Vis absorption spectroscopy and SEM, with EDS and XRD being carried out on MGN1. MGN1 was also further analysed using TEM and STEM EDS.

\subsubsection{UV-Vis Absorption Spectroscopy}

Each sample presented one peak in the visible region of the UV-Vis absorption spectra, deemed to be due to spherical gold nanoparticles present in the samples. As seen in Figure 4.26, MGN1 showed a reasonably sharp and well-defined peak at $555 \mathrm{~nm}$, MGN2 showed a broad peak at $560 \mathrm{~nm}$ and MGN3 showed a small peak at $540 \mathrm{~nm}$. All spectra have high absorbance at low wavelength due to the uncapped silica in the sample which hides some of the MGN3 peak, making it appear 
as more of a shoulder. The silica also absorbs at higher wavelength to a lesser degree, which could be masking a small secondary peak, indicative of core-shell particles.

It can be observed that the absorbance peak exhibited by MGN3 was significantly blue shifted compared to the absorbance peaks exhibited by MGN1 and MGN2. If these peaks were interpreted to be due to gold shells, this blue shift would indicate an increase in the thickness of the shells in MGN3. If these peaks were interpreted to be due to spherical gold particles, this blue shift would indicate a decrease in diameter of the spherical gold particles in MGN3. In the synthesis of gold nanoparticle colloids, increased sodium borohydride concentrations lead to larger particle diameters. ${ }^{57}$ The synthesis of MGN3 involved more sodium borohydride than the syntheses of MGN1 and MGN2; therefore it is possible that the MGN3 sample produced gold shells of greater thickness than those found in MGN1 and MGN2. However, in order for gold shells SPR absorbance to occur at such low wavelengths the shells must be of sufficient thickness to induce a lower wavelength shoulder absorption peak in the UV-Vis absorption spectra, as demonstrated by Figure 1.10. As no shoulder absorption peak is observed at lower wavelength nor is a greater intensity peak observed at higher wavelength it is considered unlikely that these peaks are due to gold shells in the samples.

As the synthesis of MGN3 involved a greater volume of sodium borohydride than the syntheses of MGN1 and MGN2 any spherical gold nanoparticles formed in solution would be of larger diameter, causing a red shift of the UV-Vis absorption peak. Hence, it can be determined that these peaks were not due to discrete spherical gold nanoparticles formed in the sample. However, as no stabiliser was used in the reactions, aggregation of spherical gold nanoparticles formed in the sample was free to occur. It is possible that aggregation occurred to a greater degree in the samples with less sodium borohydride. As smaller particles have greater surface energy, they will aggregate more readily to stabilise the system. It is possible that this increased aggregation in MGN1 and MGN2 resulted in the samples having larger aggregates of gold in the samples than in MGN3 as the gold nanoparticles formed in MGN3 were of greater diameter and would have been less likely to aggregate. 


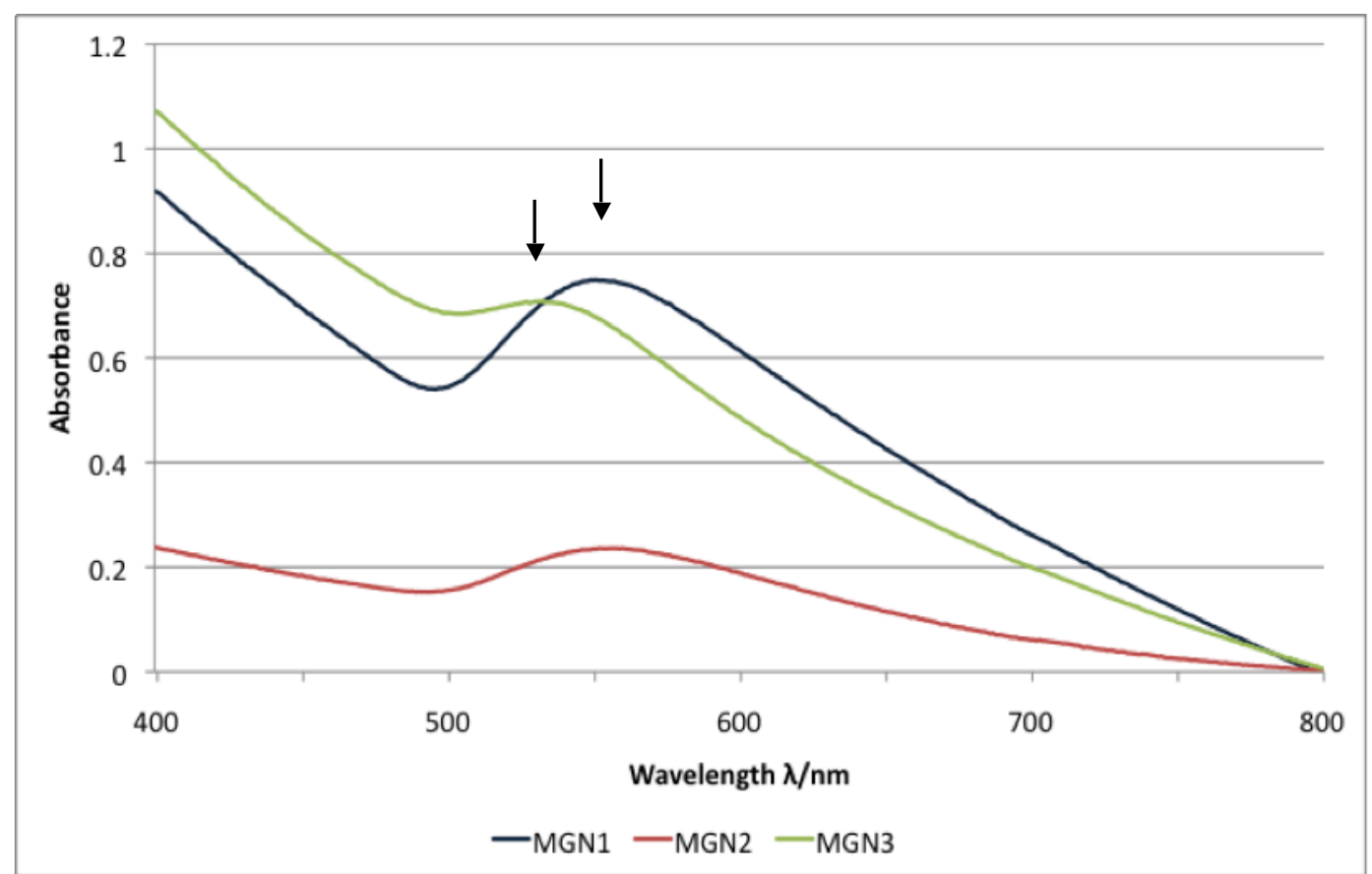

Figure 4.26: UV-Vis absorption spectra of samples MGN1, MGN2 and MGN3, synthesised via in-situ seeding on MPTMS capped silica spheres with $\mathrm{NaBH}_{4}$. Samples were not of equal concentration.

\subsubsection{SEM}

SEM was carried out on JEOL-JSM-6610LA and samples were coated with $8 \mathrm{~nm}$ of carbon. As seen in Figure 4.27, SEM imaging of MGN1 showed some areas of smooth spheres of high-density material, approximately the size of silica spheres, approximately $180 \mathrm{~nm}$ in diameter, as well as unmodified capped silica spheres. 


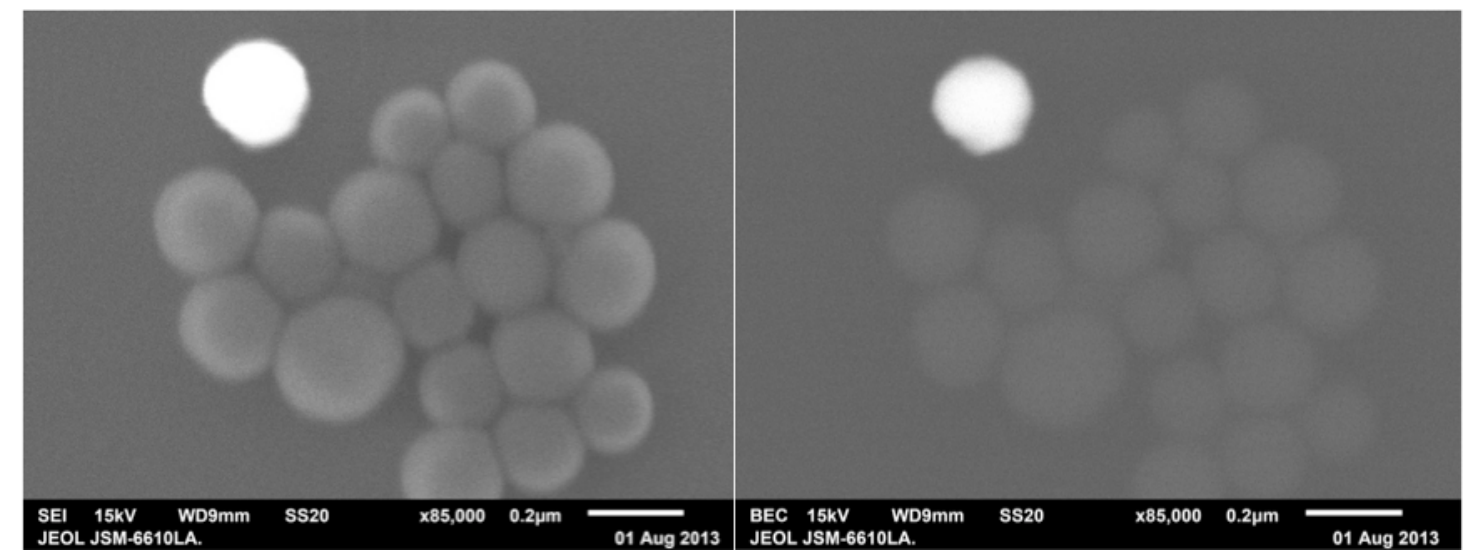

Figure 4.27: SEM images of MGN1 in both SEI (left) and backscatter (right) modes, showing smooth spheres of high-density material, of approximately the size of silica spheres.

As seen in Figure 4.28, SEM imaging of MGN2 showed some areas similar to the core-shell particles in MGN1, these areas were spherical and of similar size to those gold shells found in MGN1. However, SEM imaging also showed that the sample contained areas of high-density material that were not gold shells as they were of irregular size and shape. These areas were determined to be areas of gold metal that had been fully reduced but had not formed shells on the surface of the silica spheres.
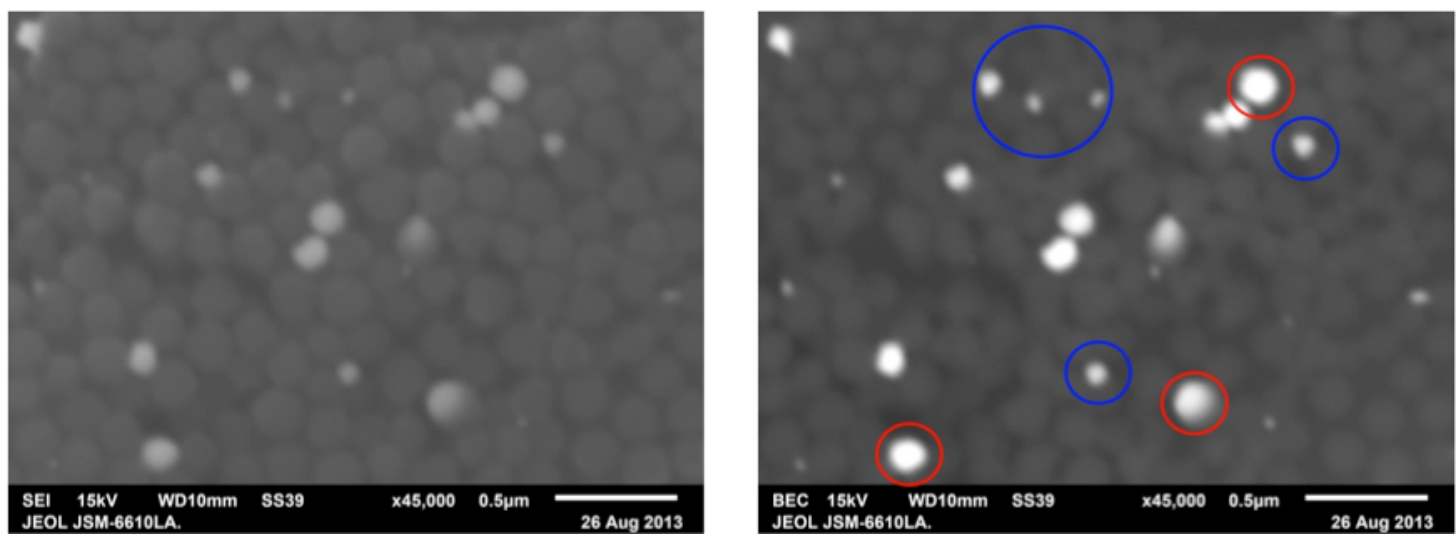

Figure 4.28: SEM images of MGN2 in both SEI (left) and backscatter (right) modes. Red circles indicate spherical gold areas similar to core-shell particles in MGN1 sample, blue circles indicate areas of gold of irregular shape and size. 
Figure 4.29 shows the EDS map of the elemental composition of an area in MGN1. This EDS analysis confirms the high-density material is gold, and also shows the presence of oxygen in the same area, indicating a silica-gold core-shell particle. As samples were drop cast onto silicon wafers, the mapping of silicon gives no useful information.

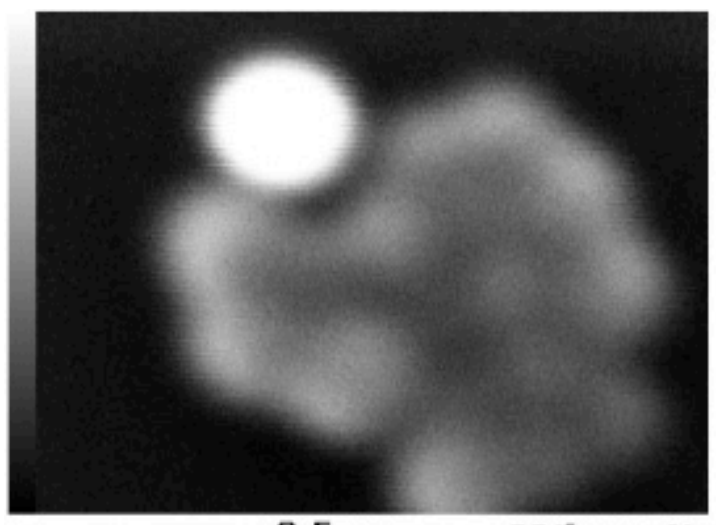

$0.5 \mu \mathrm{m}$

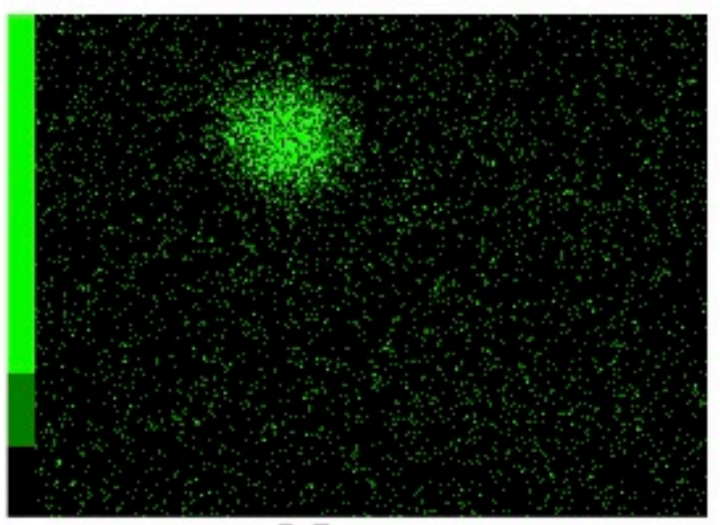

$0.5 \mu \mathrm{m}$
Au $\mathbf{M}$

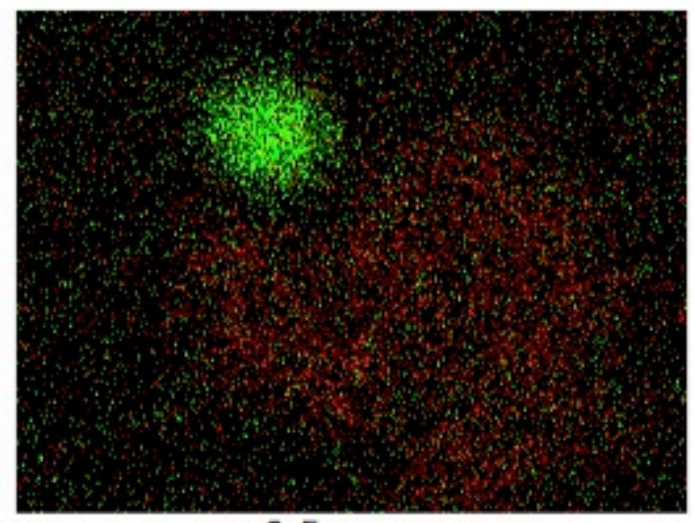

$0.5 \mu \mathrm{m}$

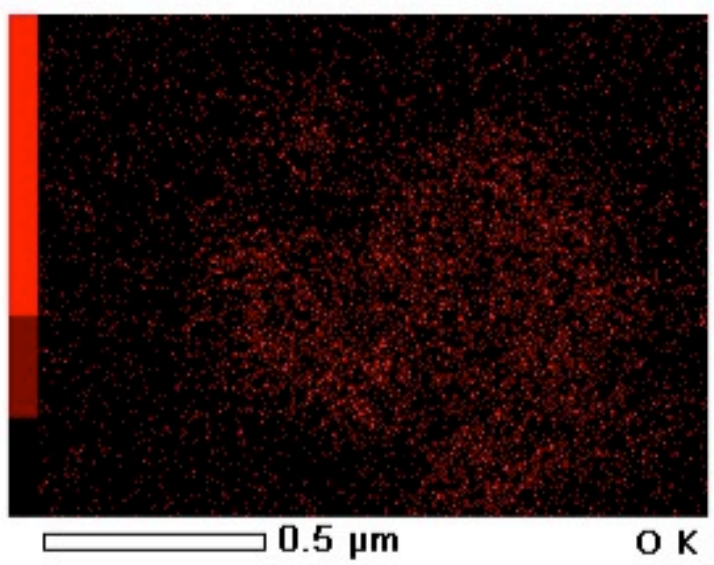

Figure 4.29: EDS mapping of MGN1 showing silica-gold core-shell particle.

Top left: Backscatter image. Bottom left: Gold map. Bottom right: Oxygen map.

Top right: Overlay of gold and oxygen elemental maps. 


\subsubsection{XRD}

XRD analysis confirmed the presence of crystalline gold metal in sample MGN1. This confirms that gold present in this sample was fully reduced to gold metal.

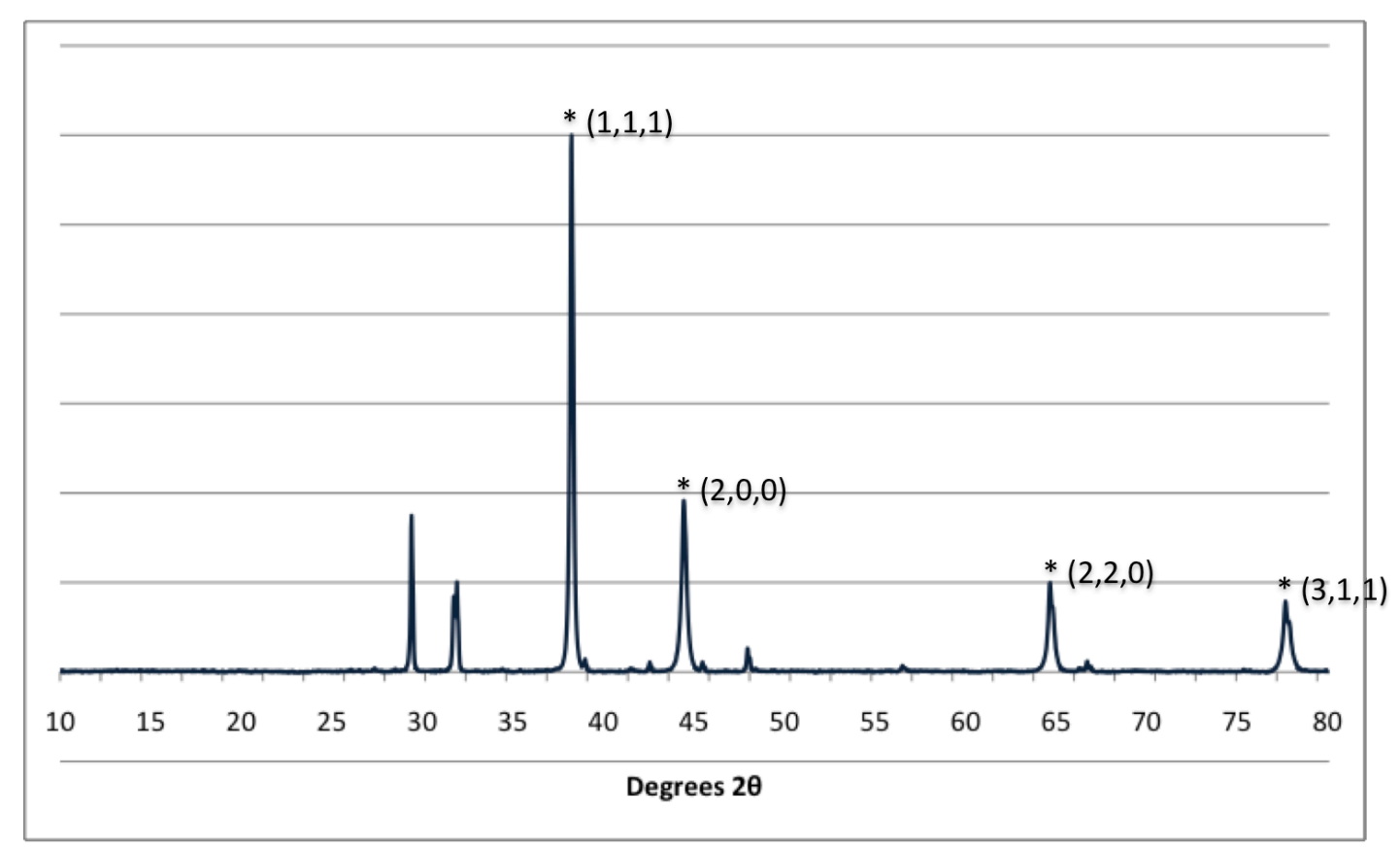

Figure 4.30: XRD spectrum of sample MGN1 showing peaks due to crystal planes of gold metal.

\subsubsection{TEM}

TEM of MGN1 showed the presence of large dense particles of reasonably spherical shape, seen as dark areas in Figure 4.31. TEM also showed these particles to be of approximately $200 \mathrm{~nm}$ in diameter. These particles were suspected to be silica-gold core-shell particles and so the elemental composition of these particles was analysed using STEM EDS. 

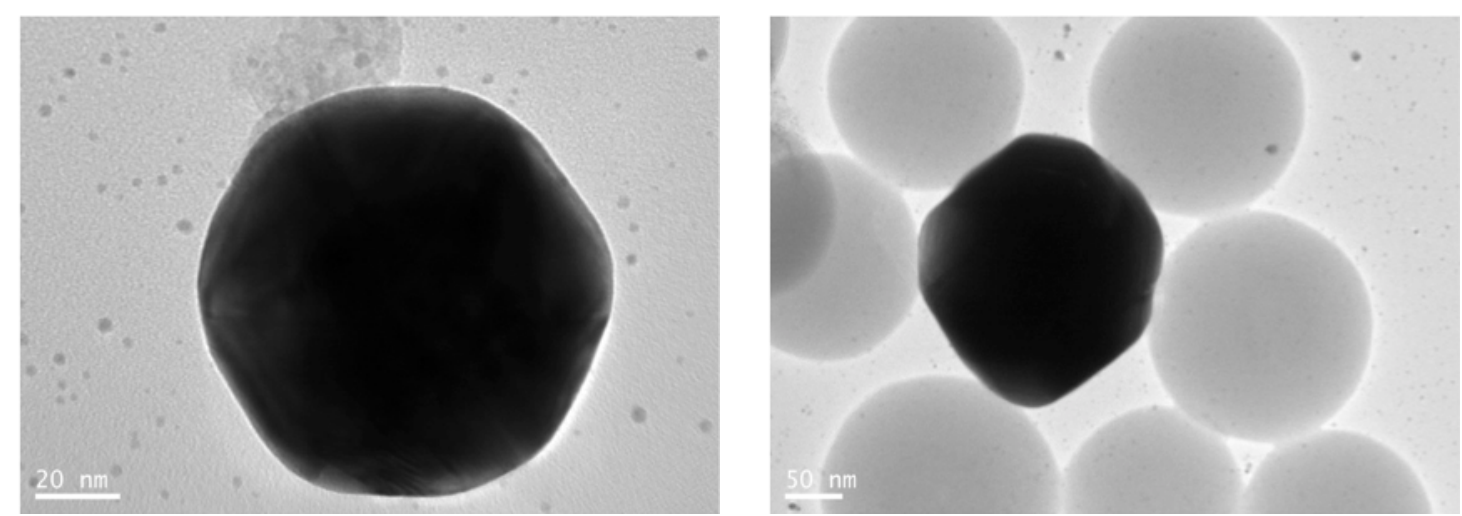

Figure 4.31: TEM images of large dense particles in sample MGN1 (dark areas), showing almost spherical shape. Silica spheres without gold shells are also seen in the image on the right.

STEM EDS analysis showed that these dense particles were composed of gold, but also showed the presence of silicon and oxygen, as shown in Figure 4.32. Due to the strength of the gold signal, the overlay image does not clearly show the silicon and oxygen signals in the same area. However, in the individual elemental maps, it is clear that both silicon and oxygen are mapped to the same area as gold. This suggests that the particles are silica-gold core-shell particles. 


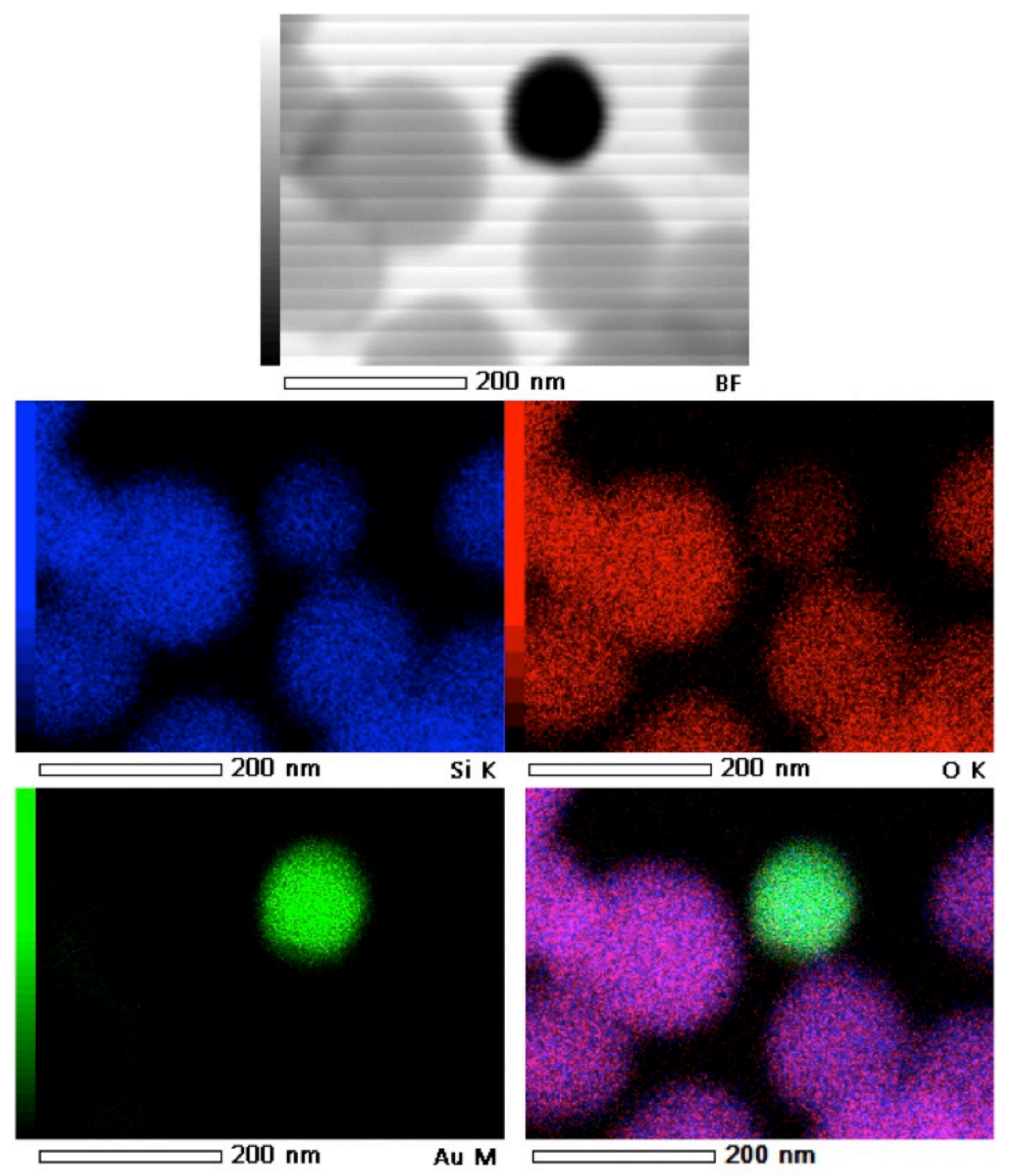

Figure 4.32: STEM EDS maps of dense particles in sample MGN1, showing presence of silicon and oxygen with gold. Top: STEM image. Middle left: Silicon map.

Middle right: Oxygen map. Bottom left: Gold map. Bottom right: Overlap of silicon, oxygen and gold elemental maps. 


\subsubsection{Summary}

The UV-Vis absorption spectra of these samples each showed only one significant peak at approximately $540-560 \mathrm{~nm}$, characteristic of spherical gold nanoparticles, with MGN3 having the lowest intensity peak at the lowest wavelength. This indicated the syntheses had been unsuccessful in forming gold shells, however the observed colours of the samples indicated that a result had occurred. SEM showed the presence of core-shell-like particles in all samples, with the core-shell particles being smoothest in MGN1 and the most concentrated in MGN2. MGN3 had some core-shell particles but the sample also contained many large areas of gold on silica spheres that had not formed shells. MGN1 was then analysed using XRD, confirming the presence of crystalline gold metal. MGN1 was deemed to have the best core-shell particles and so MGN1 was further analysed using TEM, to confirm the presence silica-gold core-shell particles.

Although none of these samples showed high wavelength peaks in UV-Vis absorption spectroscopy, SEM, XRD and TEM showed that all samples contained core-shell particles. The samples also contained many large gold nanoparticles, which could have obscured the UV-Vis absorption and concealed the SPR absorption peaks of the core-shell particles. Sample MGN1 successfully synthesised reasonably spherical core-shell particles, but there were many silica spheres in the sample left without shells. This highlights an inefficiency in the synthetic method and it was decided to further investigate this issue by altering some of the reaction parameters.

\subsection{First Adaptations of MGN1 Synthesis}

Sample MGN1 was found to have successfully formed silica-gold core-shell particles, but many capped silica spheres were left bare, without gold shells. To form more gold shells, the effects of some reaction parameters were investigated. Since there were many bare capped silica spheres, the adapted syntheses were carried out with 20 times less silica in the reaction mixture. Water was used as a solvent in one sample as this would provide a cheaper, safer synthetic method. In an effort to form more shells, the amount of gold in the seeding step was increased and the amount of 
gold in the growth steps was also increased, as was the amount of hydroxylamine solution to reduce this gold.

Samples were prepared by adding silica and $\mathrm{AuCl}_{4}{ }^{-}$together in a solvent. These samples were left at room temperature for 24 hours before $\mathrm{NaBH}_{4}$ was added and the samples were again left at room temperature, this time shaking, for 72 hours. Hydroxylamine solution was then added to the samples and they were left at $50{ }^{\circ} \mathrm{C}$ overnight. Successive aliquots of $50 \mu \mathrm{L}$ of $\mathrm{AuCl}_{4}{ }^{-}$were then added to the samples until the necessary total amount of $\mathrm{AuCl}_{4}{ }^{-}$had been delivered to each sample. Samples were left at $50{ }^{\circ} \mathrm{C}$ for at least 6 hours between additions and for 12 hours after the final addition. See Section 2.4.6 In-situ Seeding on MPTMS Capped Silica Spheres with $\mathrm{NaBH}_{4}$ and Hydroxylamine and Table 4.8 for full experimental details.

After addition of hydroxylamine solution MGN1-1W very quickly changed from translucent yellow to pink, then almost opaque orange/brown. MGN1-1a, MGN1-b and MGN1-1c all lost yellow colour but did not develop any other colour at this stage and remained white. After first subsequent addition of $\mathrm{AuCl}_{4}{ }^{-}, \mathrm{MGN1} 1 \mathrm{~W}$ had big, dark coloured clumps (approximately 0.1-0.5 mm diameter) and sediment plated on the bottom of the vial, while still looking orange/brown but had lost some opacity. MGN1-1a, MGN1-1b and MGN1-1c had similar clumps to MGN1-1W but smaller and less of them, as well as similar sediment plating the bottom of the vial. The colours of the samples had all changed to orange/brown.

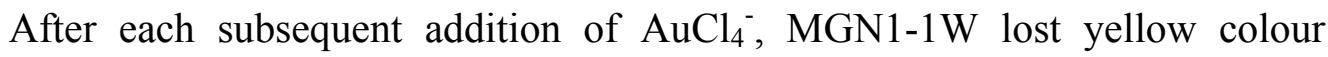
quickly and remained orange/brown throughout the synthesis. MGN1-1b lost yellow colour after first subsequent addition of $\mathrm{AuCl}_{4}{ }^{-}$but the yellow colour remained after further additions.

MGN1-1a quickly lost yellow colour after first subsequent addition of $\mathrm{AuCl}_{4}{ }^{-}$, as did MGN1-1c although the loss of colour took longer for this sample. After $100 \mu \mathrm{L}$ of $\mathrm{AuCl}_{4}^{-}$had been added to the samples the yellow colour was not lost, and $0.104 \mathrm{~mL}$ of hydroxylamine solution was added to samples MGN1-1a and MGN1-1c to reduce the remaining $\mathrm{AuCl}_{4}^{-}$. After this extra hydroxylamine solution was added the yellow colour still remained in the samples, so another $0.104 \mathrm{~mL}$ of hydroxylamine solution was added to each sample. The yellow colour was then lost 
from the samples and a final $50 \mu \mathrm{L}$ of $\mathrm{AuCl}_{4}{ }^{-}$was added. Some of the yellow colour from this final addition of $\mathrm{AuCl}_{4}{ }^{-}$remained throughout the rest of the synthesis.

The colour change observed in the samples was consistent with that observed in the original MGN1 sample. All samples were observed to appear different colours in reflected and transmitted light. In reflected light the samples appeared brown/orange and in transmitted light the samples appeared blue/purple.

Table 4.8: Samples prepared via adaptation of MGN1 synthesis. Extra hydroxylamine solution was added to samples MGN1-1 a and MGN1-1c between additions of $\mathrm{AuCl}_{4}^{-}$.

\begin{tabular}{|c|c|c|c|c|c|}
\hline $\begin{array}{c}\text { Sample } \\
\text { name }\end{array}$ & Solvent & $\begin{array}{c}\text { Volume of } \\
\text { MPTMS } \\
\text { silica }\end{array}$ & $\begin{array}{c}\text { Initial } \\
\text { volume of } \\
\text { AuCl }_{4}^{-} \\
\text {solution } \\
\quad(\mu L)\end{array}$ & $\begin{array}{c}\text { Added } \\
\text { volume of } \\
\text { AuCl }_{4}^{-} \\
\text {solution } \\
\quad(\mu L)\end{array}$ & $\begin{array}{c}\text { Volume of } \\
\text { hydroxylamine } \\
\text { solution }(m L)\end{array}$ \\
\hline MGN1 & $\begin{array}{l}9.0 \mathrm{~mL} \\
\text { ethanol }\end{array}$ & $1.0 \mathrm{~mL}$ & 50 & $2 \times 50$ & 0.209 \\
\hline MGN1-1W & $10 \mathrm{~mL}$ water & $50 \mu \mathrm{L}$ & 50 & $2 \times 50$ & 0.209 \\
\hline MGN1-1a & $10 \mathrm{~mL}$ ethanol & $50 \mu \mathrm{L}$ & 50 & $3 \times 50$ & $0.209+2 \times 0.104$ \\
\hline MGN1-1b & $10 \mathrm{~mL}$ ethanol & $50 \mu \mathrm{L}$ & 100 & $2 \times 50$ & 0.209 \\
\hline MGN1-1c & $10 \mathrm{~mL}$ ethanol & $50 \mu \mathrm{L}$ & 100 & $3 \times 50$ & $0.209+2 \times 0.104$ \\
\hline
\end{tabular}

\subsubsection{UV-Vis Absorption Spectroscopy}

UV-Vis absorption spectra of all samples were obtained. All samples showed a very minor peak at approximately $710 \mathrm{~nm}$ and a shoulder peak at approximately 
580-600 nm. This small absorption at higher wavelength was indicative of successful synthesis of gold shells, while the peaks at lower wavelengths were determined to be caused by spherical gold nanoparticles also present in the samples, red shifted due to the higher refractive index of silica.

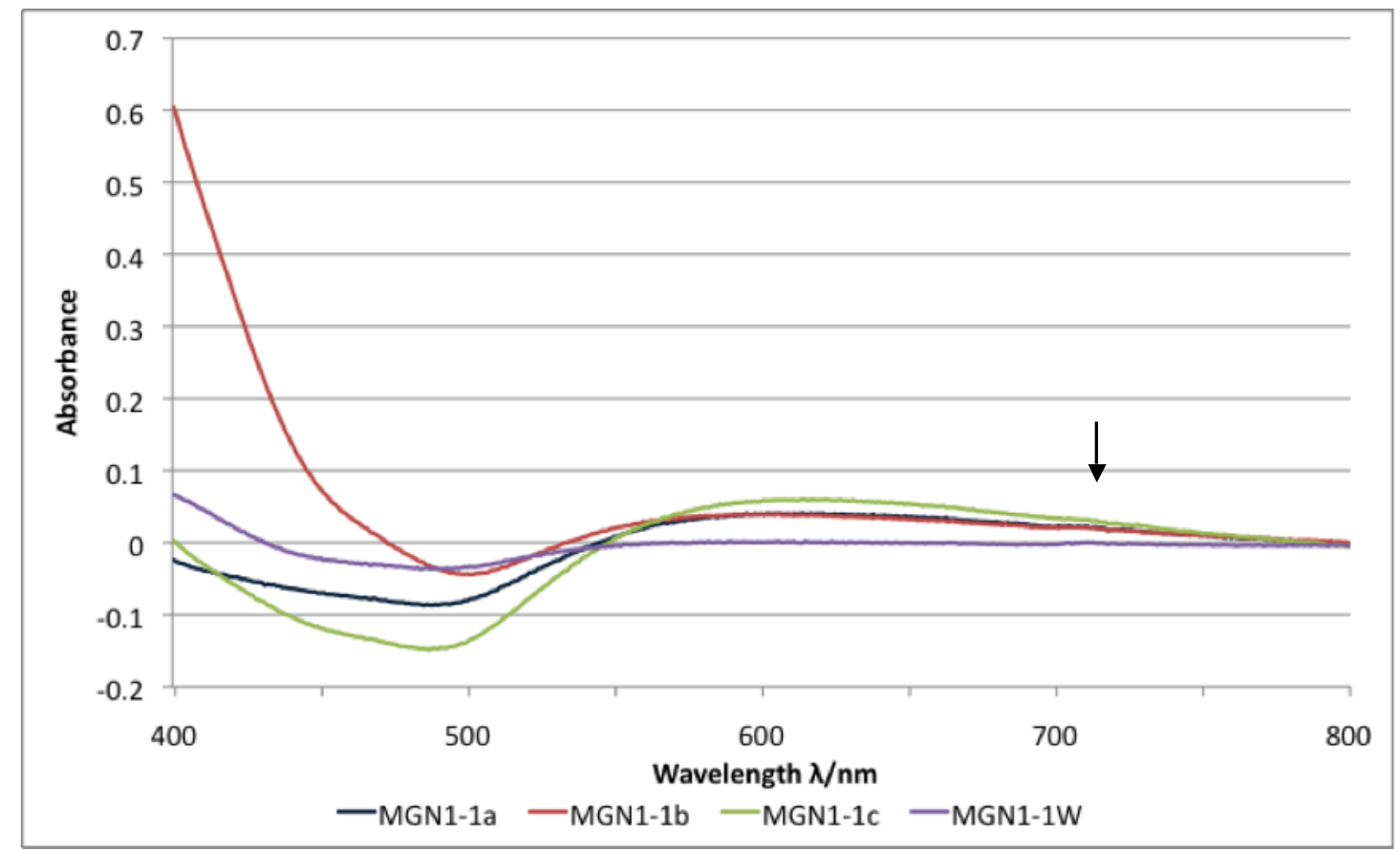

Figure 4.33: UV-Vis absorption spectra of samples prepared by first adaptations of MGN1 synthesis.

\subsubsection{SEM}

SEM was carried out on JEOL-JSM-6610LA instrument and all samples were coated with $8 \mathrm{~nm}$ of carbon. As seen in Figure 4.34, sample MGN1-1a contained many large areas of gold on top of silica sphere aggregates. These large areas of gold were also found in samples MGN1-1b, MGN1-1c and MGN1-1W, while smaller spherical particles of diameter approximately $200 \mathrm{~nm}$ were also found in MGN1-1b, MGN1-1c and MGN1-1W, as shown in Figure 4.35. These smaller spherical particles were likely core-shell particles and this was investigated using EDS mapping. 


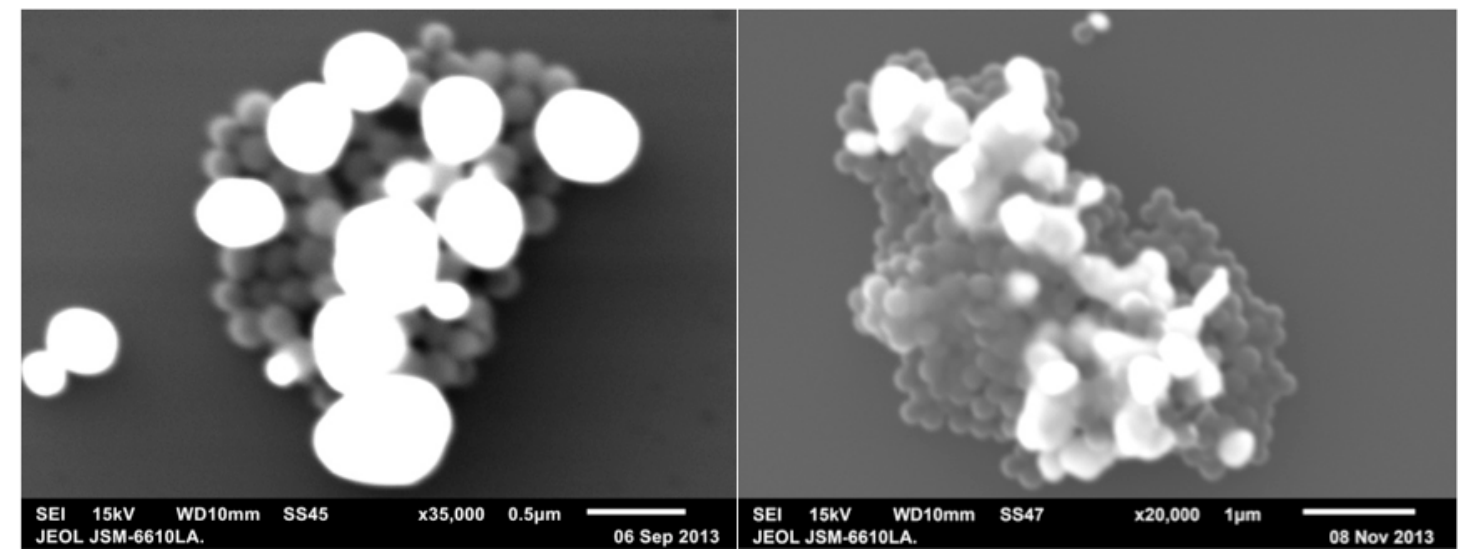

Figure 4.34: SEM images of MGN1-1 a (left) and MGN1-1W (right) in SEI mode, showing large blobby areas of gold on silica sphere aggregates.
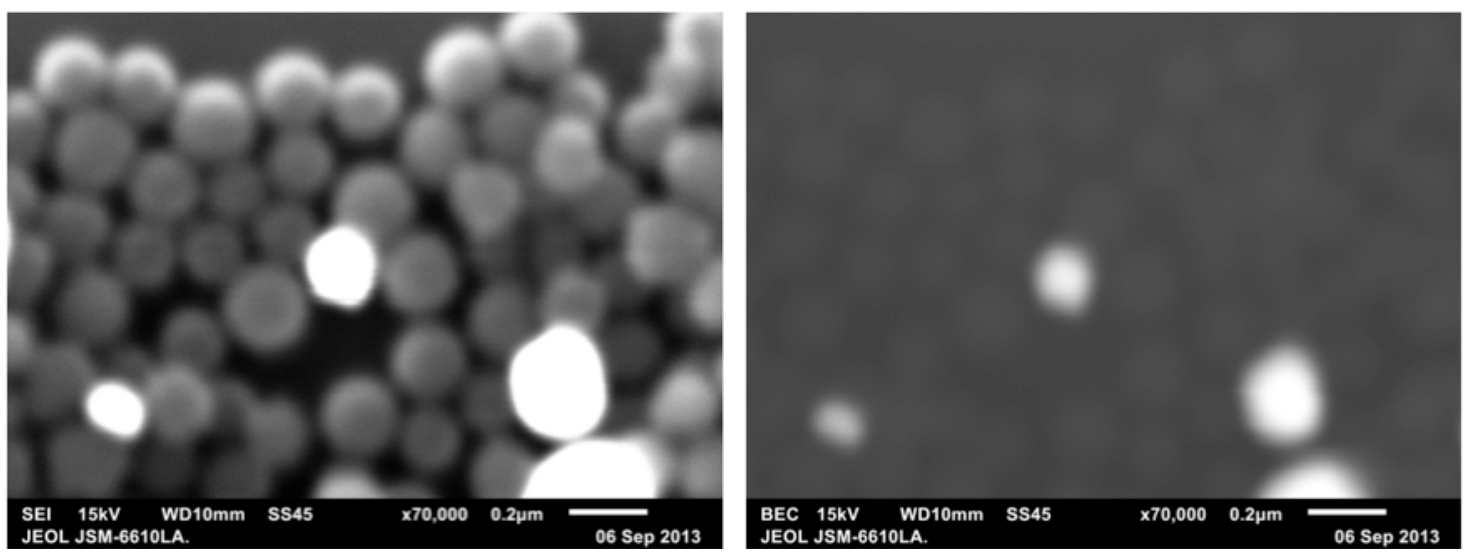

Figure 4.35: SEM images of MGN1-1b in both SEI (left) and backscatter (right) modes, showing particles that look like core-shells (bright round areas).

EDS mapping of sample MGN1-1b showed that oxygen was present in the same areas as the spherical gold particles thought to be core-shells. This indicates that 
the particles are silica-gold core-shell particles. EDS mapping was not carried out on MGN1-1c, but it is likely that the similar spherical gold particles are also silica-gold core-shell particles.
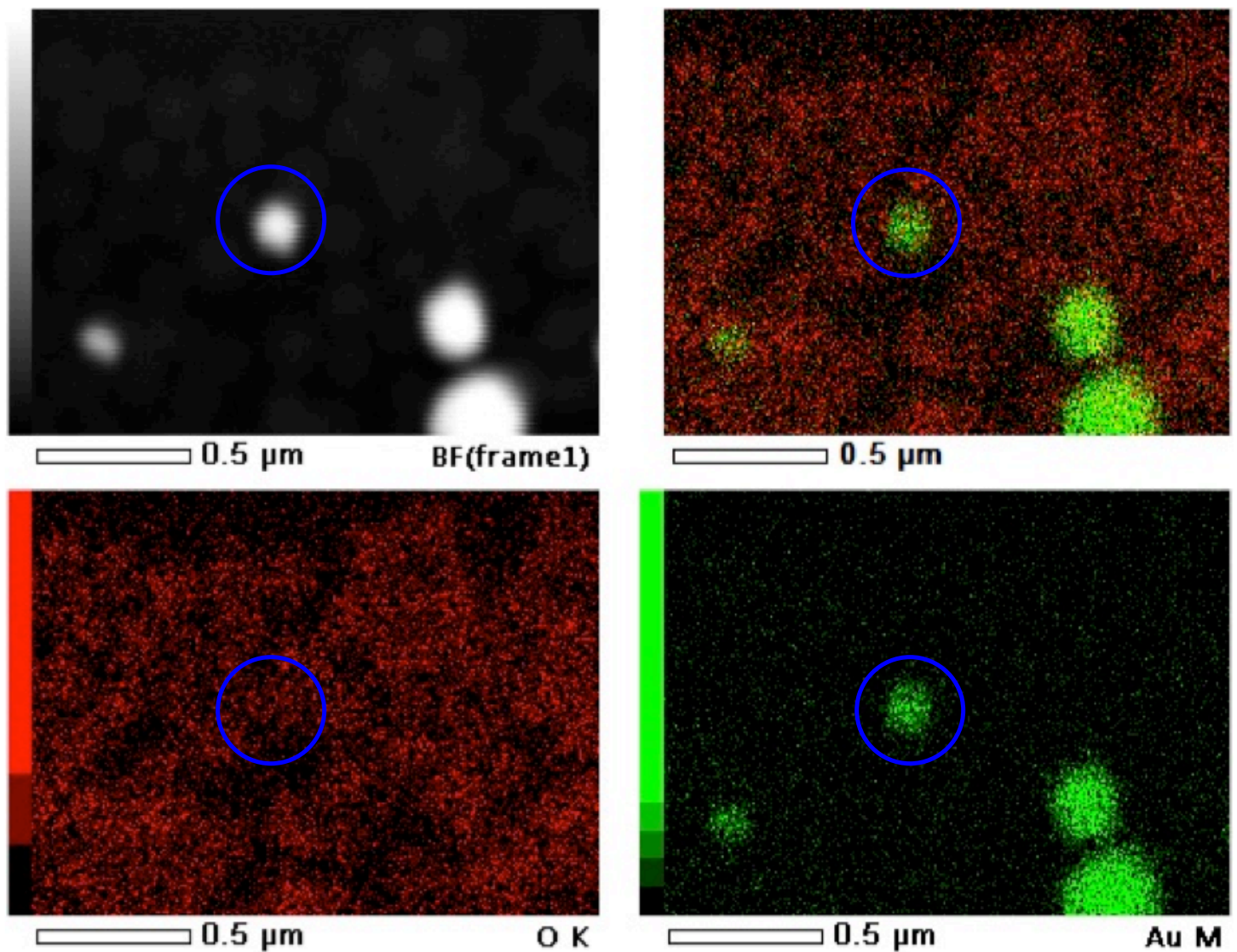

Figure 4.36: EDS map of MGN1-1b showing silica-gold core-shell particle, circled in blue. Top left: Backscatter image. Bottom left: Oxygen map. Bottom right: Gold map.

Top right: Overlay of oxygen and gold elemental maps.

\subsubsection{Summary}

As seen in Figure 4.33, UV-Vis absorption spectroscopy showed that all samples presented two peaks in the visible region, suggesting the successful formation of core-shell particles along with the presence of spherical gold nanoparticles. SEM imaging showed MGN1-1a had large irregular shaped areas of gold on silica sphere aggregates, but no core-shell particles were found. MGN1-1b was also found to have these large areas of gold on silica sphere aggregates but 
additionally had some particles that looked like core-shells. MGN1-1c was found to be similar to MGN1-1b, as it contained both irregular shaped areas of gold on silica sphere aggregates and particles that looked like core-shells, but there were much fewer potential core-shell particles in this sample than in MGN1-1b. MGN1-1W was also found to have formed some clumpy areas of gold on silica spheres and some potential core-shell particles. EDS mapping of MGN1-1b supported the notion that the sample contained core-shell particles as oxygen was found to map to the same area as gold.

\subsection{Second Adaptations of MGN1 Synthesis}

Further investigation into the reaction parameters of MGN1 synthesis was carried out after initial investigations were unsuccessful in overcoming the problems in the MGN1 sample. After the first adaptations of MGN1 synthesis involving lower concentrations of capped silica spheres yielded large masses of gold on silica that did not form spheres, the concentration of capped silica was increased to the original concentration of the MGN1 synthesis, and other reaction parameters were altered as summarised in Table 4.9.

Samples were prepared by combining MPTMS capped silica in ethanol with $\mathrm{AuCl}_{4}{ }^{-}$and leaving for 24 hours at $50{ }^{\circ} \mathrm{C}$. After this time, $\mathrm{NaBH}_{4}$ was added to the samples and they were left on a shaker table overnight at room temperature. At this point, one sample was washed with ethanol. The next day, $0.209 \mathrm{~mL}$ of hydroxylamine solution was added to the samples and they were left at $50{ }^{\circ} \mathrm{C}$. Two further additions of $50 \mu \mathrm{L}$ of $\mathrm{AuCl}_{4}{ }^{-}$were added to the samples. Samples were kept at $50{ }^{\circ} \mathrm{C}$ before and after each addition of $\mathrm{AuCl}_{4}{ }^{-}$. 
Table 4.9: Samples prepared by second adaptations of original MGN1 synthesis.

\begin{tabular}{|c|c|c|c|c|}
\hline Sample name & $\begin{array}{c}\text { Volume of } \\
\text { MPTMS silica } \\
\text { (mL) }\end{array}$ & $\begin{array}{l}\text { Initial volume } \\
\text { of } \mathrm{AuCl}_{4}^{-} \\
\text {solution }(\mu L)\end{array}$ & $\begin{array}{c}\text { Time and } \\
\text { temperature left } \\
\text { before addition } \\
\text { of } \mathrm{NaBH}_{4}\end{array}$ & $\begin{array}{c}\text { Washing of } \\
\text { reaction } \\
\text { mixture after } \\
\text { addition of } \\
\mathrm{NaBH}_{4}\end{array}$ \\
\hline MGN1 & 1.0 & 50 & 3 hours@RT & No \\
\hline MGN1a & 1.0 & 50 & 24 hours @ $50^{\circ} \mathrm{C}$ & No \\
\hline MGN1b & 1.0 & 50 & 24 hours @ $50^{\circ} \mathrm{C}$ & Yes \\
\hline MGN1c & 1.0 & 40 & 24 hours $@ 50{ }^{\circ} \mathrm{C}$ & No \\
\hline MGN1d & 0.5 & 40 & 24 hours @ $50{ }^{\circ} \mathrm{C}$ & No \\
\hline
\end{tabular}

Upon washing sample MGN1b after addition of $\mathrm{NaBH}_{4}$, the first supernatant had a yellow colour. Yellow colour in the samples of these syntheses was attributed to $\mathrm{AuCl}_{4}{ }^{-}$Therefore, in the washing process all $\mathrm{AuCl}_{4}{ }^{-}$that was not reduced by $\mathrm{NaBH}_{4}$ was lost. Hence, MGN1b had less gold in the total synthesis than the original MGN1 synthesis. The solid obtained in the washing process was slightly purple in colour. This colour changed to orange/brown, with a slight purple, by the end of the synthesis. After the addition of hydroxylamine, the solutions had a red/orange precipitate and a transparent solution. This colour persisted to the end of the syntheses. The final colour of all samples is shown in Figure 4.37. Again, all samples were observed to appear brown/orange in reflected light and blue/purple in transmitted light. 


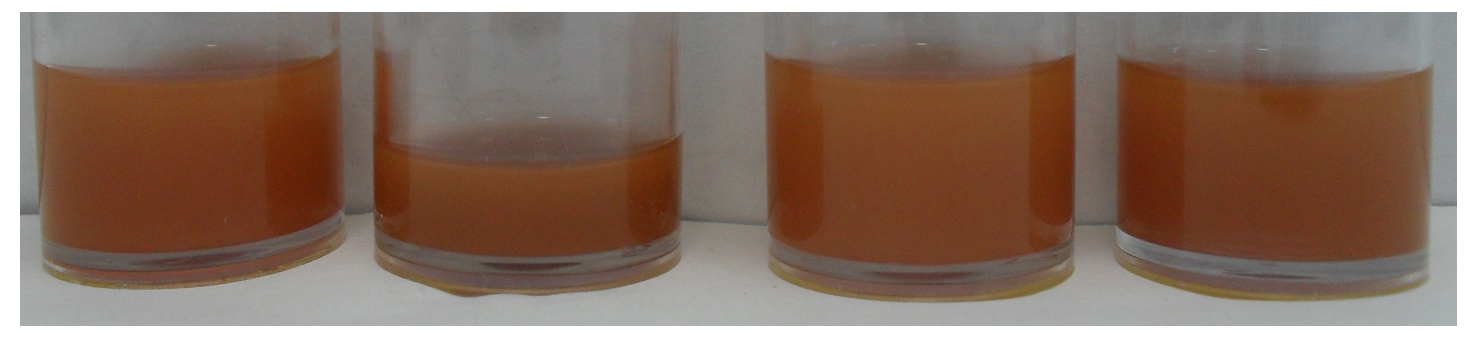

Figure 4.37: Photograph of samples MGN1a, MGN1b, MGN1c and MGN1d (left to right).

\subsubsection{UV-Vis Absorption Spectroscopy}

UV-Vis absorption spectroscopy showed that each sample had only one absorption peak with significant intensity at approximately $565 \mathrm{~nm}$, as shown by Figure 4.38. This peak was attributed to spherical gold nanoparticles present in the sample, and was determined to have been red shifted due to the higher refractive index of silica. Although no absorption peaks were observed at higher wavelengths, which would be indicative of the presence of core-shell particles, the results were similar to those obtained for the original MGN1 sample and it was determined that the SPR absorption peaks of the core-shell particles in these samples were concealed by the broad absorption of other components in the samples, such as silica spheres and large gold particles. 
Table 4.10: Wavelengths of major absorption peaks in UV-Vis absorption spectra of samples prepared by second adaptations of MGN1 synthesis.

Sample name

MGN1a

MGN1b

MGN1c

MGN1d
Major peak wavelength $\left(\lambda_{\max } / \mathrm{nm}\right)$

$$
560
$$

570

565

565

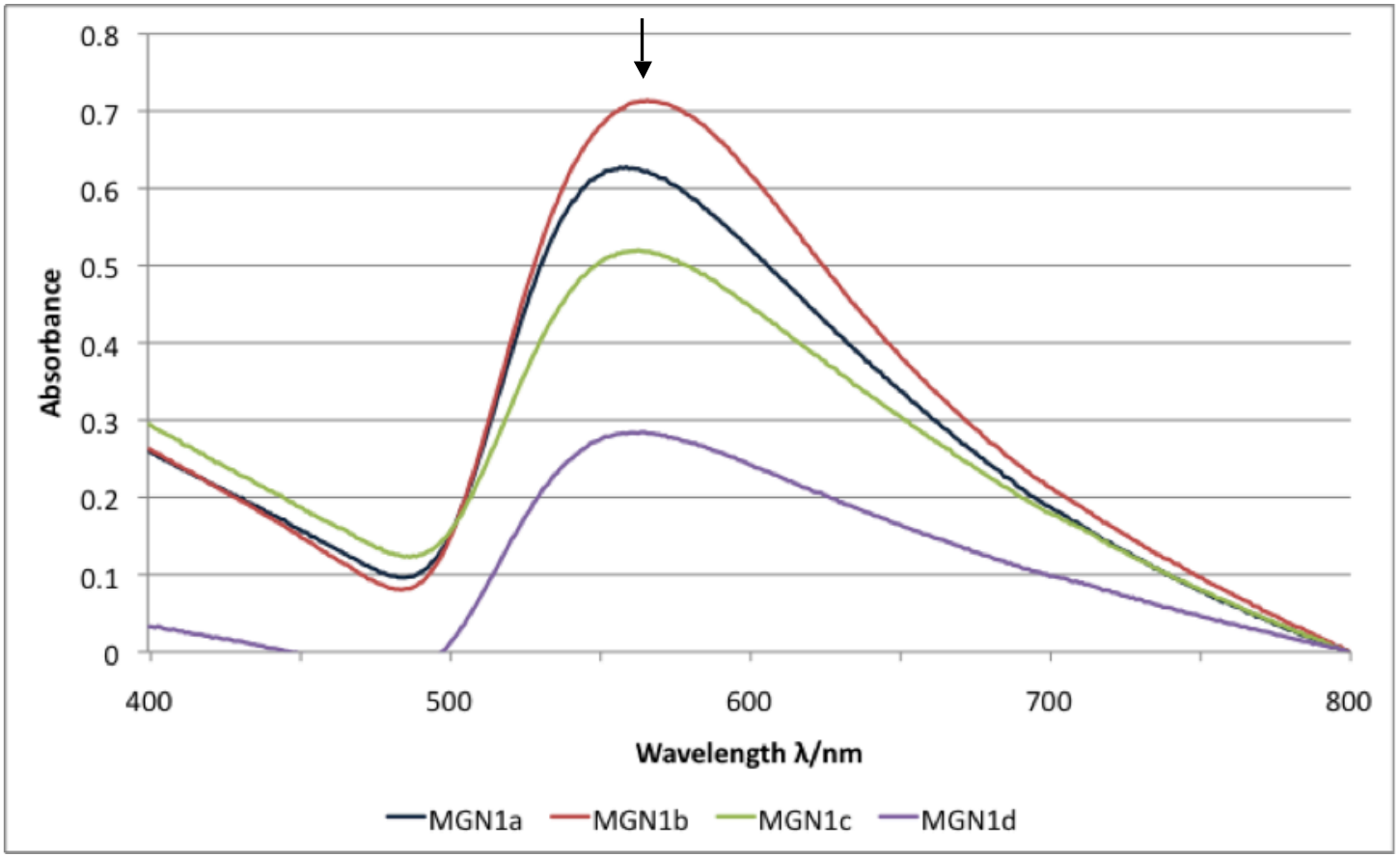

Figure 4.38: UV-Vis absorption spectra of samples prepared by second adaptations of MGN1 synthesis. Samples were not of equal concentrations. 


\subsubsection{SEM}

All samples were coated with $8 \mathrm{~nm}$ of carbon and imaged using JEOL-JSM-6610LA SEM. EDS analysis was carried out on all samples, also using the JEOL-JSM-6610LA SEM. Sample MGN1a was found to have formed many core-shell particles. However some areas of large gold agglomerates on top of silica sphere aggregates were also present in the sample. Sample MGN1b was found to have formed the most core-shell particles, seen in Figure 4.39, but a few gold agglomerates were also found in this sample. Samples MGN1c and MGN1d were found to have many large gold agglomerates and only a few core-shell particles. The core-shell particles in these samples were found to be slightly larger, with diameter of approximately $220 \mathrm{~nm}$.
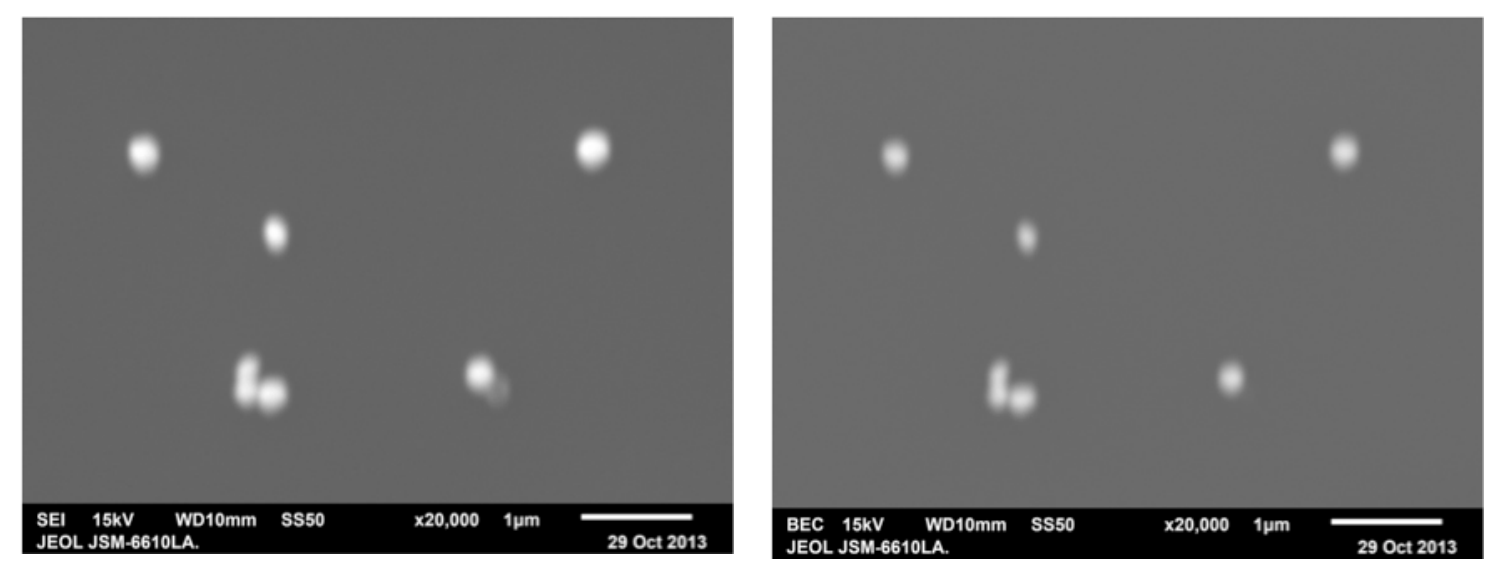

Figure 4.39: SEM images of sample MGN1b in both SEI (left) and backscatter (right) modes, showing several core-shell particles.

All samples were also analysed using EDS. EDS mapping determined oxygen was present in the areas deemed to be core-shell particles due to their size and shape during imaging (see Figure 4.40). Large agglomerates of gold were found to have a significantly weaker oxygen signal in the area, confirming that they are agglomerates of gold and not aggregates of core-shell particles. 


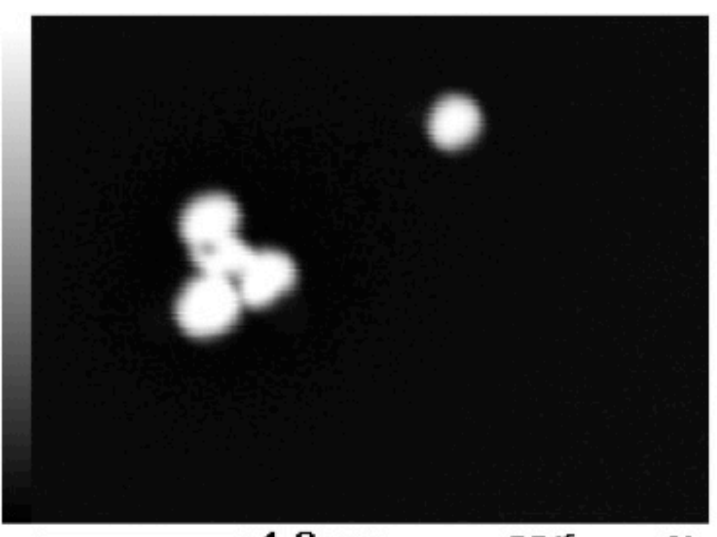

$1.0 \mu \mathrm{m}$

BF(frame1)
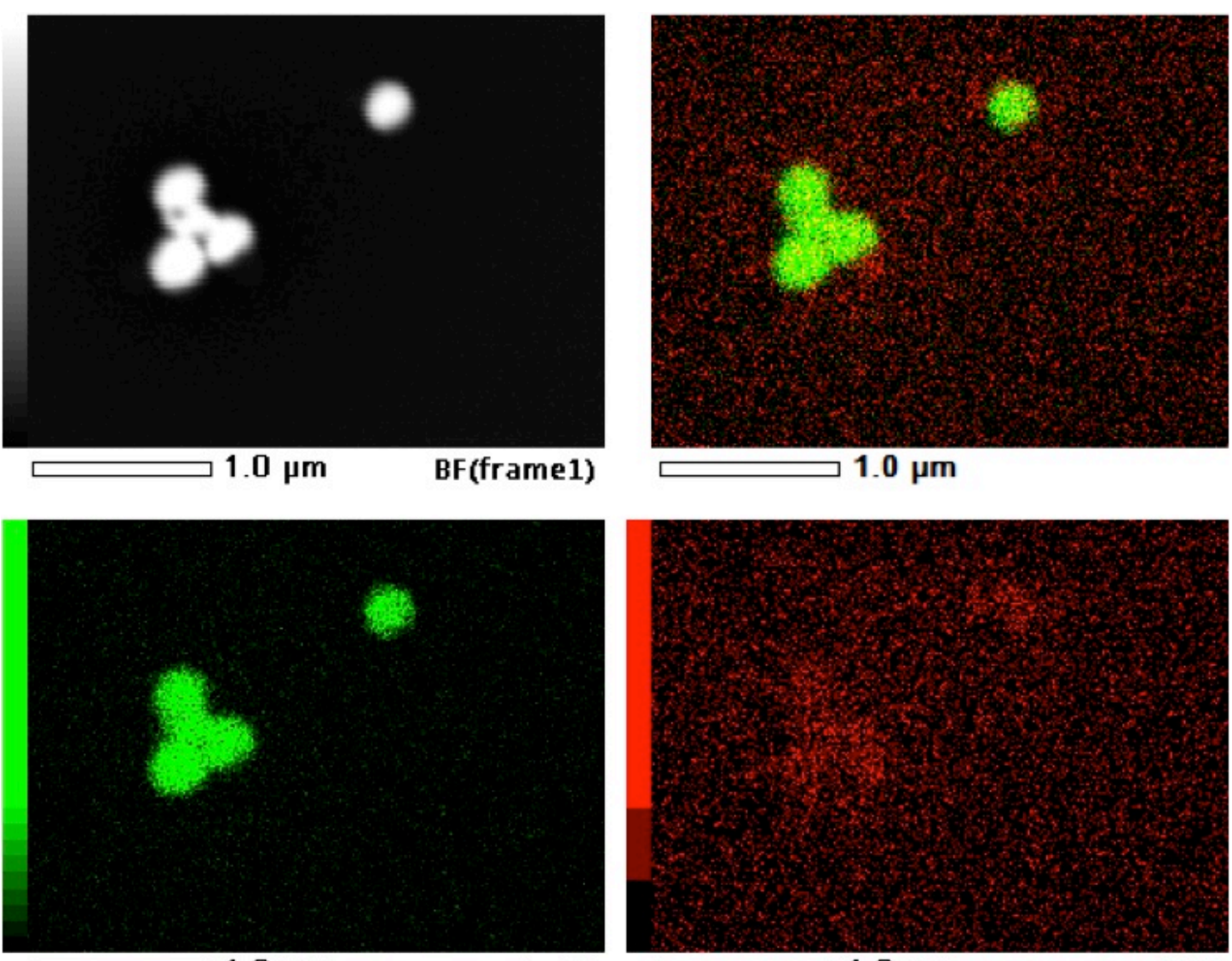

$1.0 \mu \mathrm{m}$

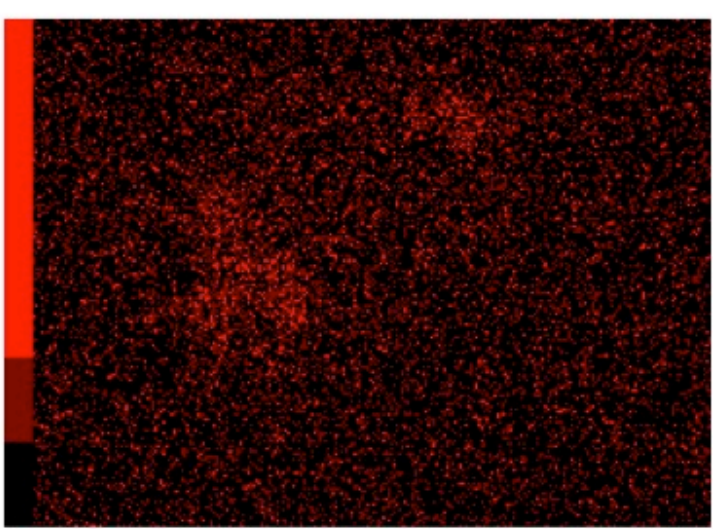

$1.0 \mu \mathrm{m}$

Figure 4.40: EDS mapping of sample MGN1c, showing presence of oxygen in the areas deemed to be core-shell particles. Top left: Backscatter image.

Bottom left: Gold map. Bottom right: Oxygen map. Top right: Overlay of gold and oxygen elemental maps.

\subsubsection{XRD}

XRD analysis confirmed the presence of crystalline gold metal in sample MGN1b. This confirms that the gold shells were reduced fully to gold metal. 


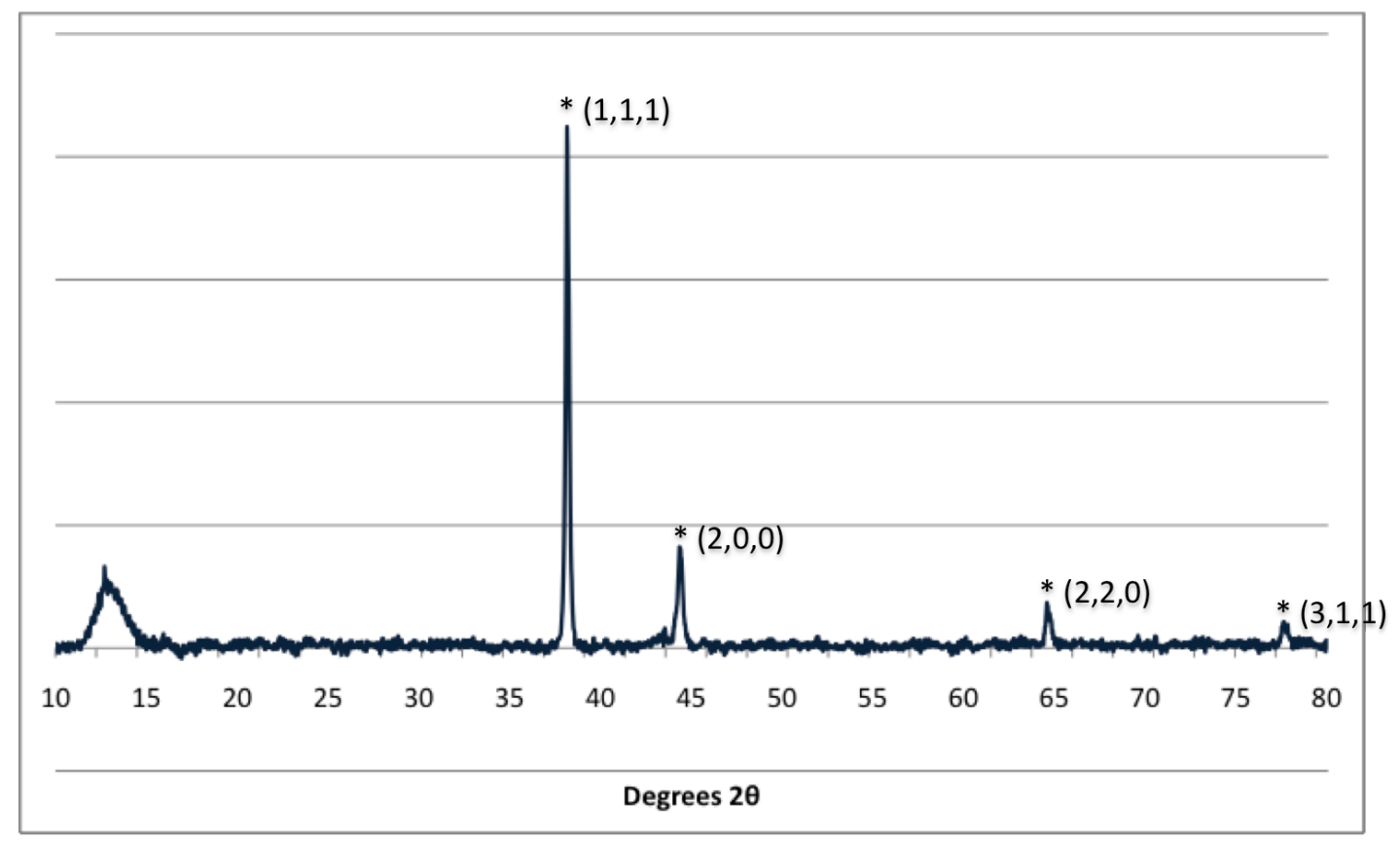

Figure 4.41: XRD spectrum of sample MGN1b showing peaks due to crystal planes of gold metal.

\subsubsection{Summary}

Samples were analysed using UV-Vis absorption spectroscopy and SEM. The UV-Vis absorption spectra of the samples showed they each had an intense absorption peak at approximately $565 \mathrm{~nm}$ due to spherical gold nanoparticles, again red shifted due to the higher refractive index of silica, but no noticeable second peak at higher wavelength. The UV-Vis absorption spectra of these samples were similar to the UV-Vis absorption spectrum of the original MGN1 sample in that they did not indicate the presence of core-shell particles. However, using SEM imaging and EDS analysis, the original MGN1 was in fact found to have successfully synthesised core-shell particles, hence these samples were further analysed. SEM showed that all samples contained core-shell particles, confirmed with EDS analysis. Samples MGN1c and MGN1d were found to have many areas of large gold agglomerates on top of silica sphere aggregates. Samples MGN1a and MGN1b were found to have many core-shell particles, with MGN1b having the most core-shells and least bare 
MPTMS capped silica spheres of any sample synthesised in this research. XRD analysis was also completed to confirm the crystallinity of the gold shells.

\subsection{Conclusions from Silica-gold Particles}

It was found that MPTMS was a better capping agent to use in the synthesis of gold shells. No gold shells were formed on the PEI capped silica, however many different synthetic methods proved successful in synthesising gold shells on MPTMS capped silica spheres.

Silica-gold core-shell particles were successfully synthesised using different synthetic schemes. The most significant problems encountered in the syntheses were the formation of large gold agglomerates and failure to form gold shells on all of the silica cores in the sample. Many parameters were altered in efforts to solve these problems. The volume of gold used in both seeding and growth of the gold shells, the volume of silica used in the synthesis, the reducing agents and reaction times were some parameters investigated. It was found that increasing the volume of gold used or decreasing the amount of silica used in the synthesis, increased the amount of gold agglomerates formed in the samples. However if too little gold was used, the samples were found to contain a high number of silica particles without gold shells. The most successful synthesis was MGN1b, which had the highest concentration of core-shells in the sample and fewest areas of gold agglomerates. This synthesis involved increased reaction times, as well as an additional washing step after reduction by sodium borohydride. This additional washing step proved to be crucial as it is supposed that this reduced the amount of gold reduced into the sample, thereby increasing the amount of gold reduced onto the silica spheres and reducing the amount of gold available to form large gold aggregates.

Most gold shells synthesised in this research had approximate diameters of either $375 \mathrm{~nm}$ or $200-220 \mathrm{~nm}$. Syntheses involving higher temperatures and no external reducing agents yielded larger particle sizes, whereas syntheses involving lower temperatures and external reducing agents, hydroxylamine and sodium borohydride, yielded smaller particle sizes. As the silica cores were the same size for all syntheses, this increased particle size indicates thicker shells. Theoretically, this could be investigated using UV-Vis absorption spectra as thicker shells should have 
red shifted SPR bands. However, the UV-Vis absorption spectra of samples synthesised at high temperatures without external reducing agents did not show a peak indicative of gold shells so this cannot be tested. The concentration of core-shell particles in these samples were quite low, hence it is supposed that the gold shell SPR peaks were too small to be observed over the background absorbance of the core material.

The colloids of core-shell particles were coloured. The colloids appeared orange/brown in reflected light and purple in transmitted light. The UV-Vis absorption spectra of the core-shell particles synthesised show that the particles have the potential to allow for a new colour to be obtained using these gold nanostructures as colourants in wool. 


\section{Colouring of Wool}

\subsection{Spherical Particles as Colourants}

Three samples shown in Figure 5.1 were prepared using proprietary methods (see Section 2.5 Colouring Wool with Gold Nanoparticles), to obtain pink, purple and grey samples. These samples were prepared for comparison to the colour achieved when using core-shell particles as colourants.

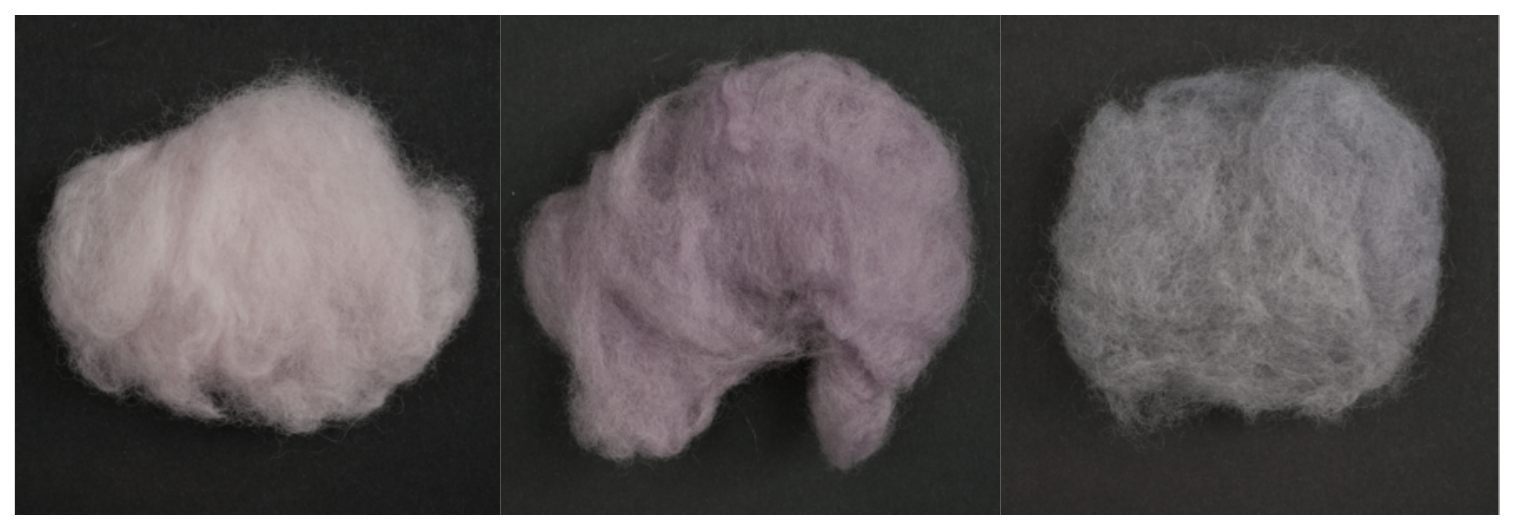

Figure 5.1: Photographs of wool samples coloured pink, purple and grey with spherical gold nanoparticles.

\subsubsection{Colour Measurements}

As shown in Figure 5.2, the Kubelka-Munk transformed reflectance spectra of wool coloured with spherical gold nanoparticles demonstrates SPR characteristics agreeing with theory. All transformed spectra show peaks at just above $400 \mathrm{~nm}$, including that of the uncoloured wool, showing that this is due to the wool itself and not the gold nanoparticles. The pink coloured wool sample has one peak, due to one size of spherical gold nanoparticles. The purple coloured wool sample has a single broader peak due to a few different sized agglomerates of spherical gold 
nanoparticles. The grey coloured wool sample has a very broad peak across the visible range due to many agglomerates of spherical gold nanoparticles of different sizes and shapes.

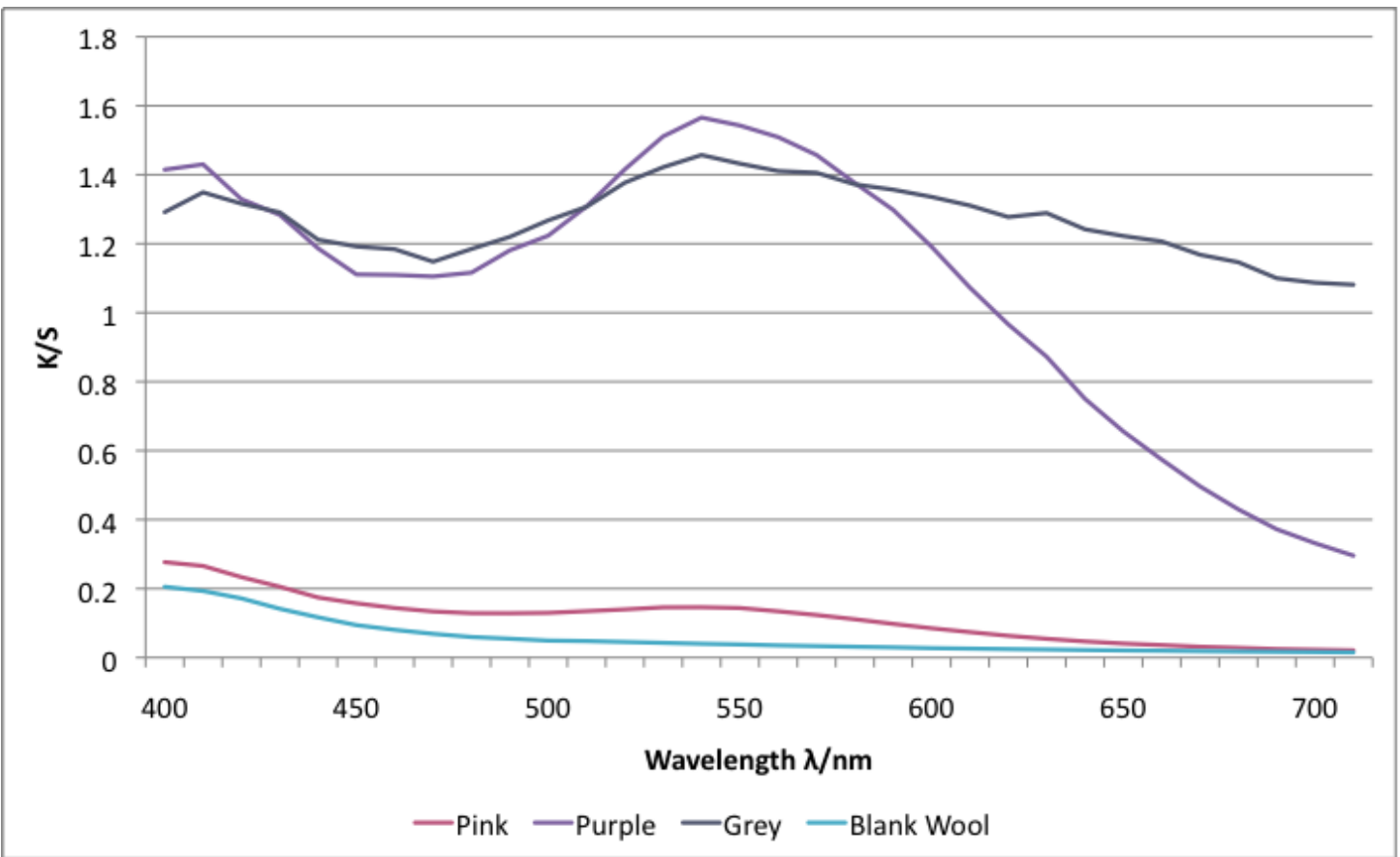

Figure 5.2: Kubelka-Munk transformed reflectance spectra of uncoloured wool and wools coloured with spherical gold nanoparticles.

The CIE Lab values of the uncoloured wool and wool samples coloured with spherical gold nanoparticles are presented in Table 5.1. The $\mathrm{L}^{*}$ value effectively represents lightness, hence the results have the expected trend of the pink sample having the highest $\mathrm{L}^{*}$ value and the grey sample having the lowest $\mathrm{L}^{*}$ value. It should be noted that the $\mathrm{L}^{*}$ value of the purple sample is very close to that of the grey sample however, the $a^{*}$ values of the samples are very different, with the purple sample having a more positive $a^{*}$ value indicating it is more red. The $b^{*}$ values of these samples are also intuitive, as the pink sample has a positive $b^{*}$ value, indicating a more yellow colour that was present in the uncoloured wool, while the purple and grey samples have negative $b^{*}$ values, indicating a more blue colour. All samples have positive $\mathrm{a}^{*}$ values, indicating they are all more red than green. 
Table 5.1: CIE Lab values for uncoloured wool and wool coloured pink, purple and grey using spherical gold nanoparticles.

$\begin{array}{cccc}\text { Sample colour } & L^{*} \text { value } & a^{*} \text { value } & b^{*} \text { value } \\ \text { Uncoloured } & 89.77 & -0.41 & 9.36 \\ \text { Pink } & 82.78 & 4.17 & 4.51 \\ \text { Purple } & 54.89 & 6.64 & -2.76 \\ \text { Grey } & 54.39 & 1.45 & -2.71\end{array}$

\subsubsection{SEM}

SEM was carried out on the JEOL-JSM-6610LA instrument after samples were coated with $16 \mathrm{~nm}$ of carbon. SEM imaging was found to damage the wool fibres, causing holes to form in the fibres when exposed to the electron beam for extended periods (seen in Figure 5.9). SEM of wool coloured with spherical nanoparticles shows a tendency for the particles to adsorb on to the wool around the cuticle edges of the fibres. This is due to the increased Gibbs free energy of these sites. As seen in Figure 5.3, SEM imaging of wool coloured pink by spherical nanoparticles shows the lowest concentration of gold nanoparticles on the surface of the wool fibres with some aggregation. However, this aggregation is limited and the particles remain discrete. The SEM images of wool coloured purple by spherical gold nanoparticles show a higher concentration of gold nanoparticles on the surface of the wool and more aggregation than in the pink wool sample. The SEM images of wool coloured grey by spherical gold nanoparticles show the highest concentration of gold nanoparticles with many aggregates of different sizes on the surface of the wool. 

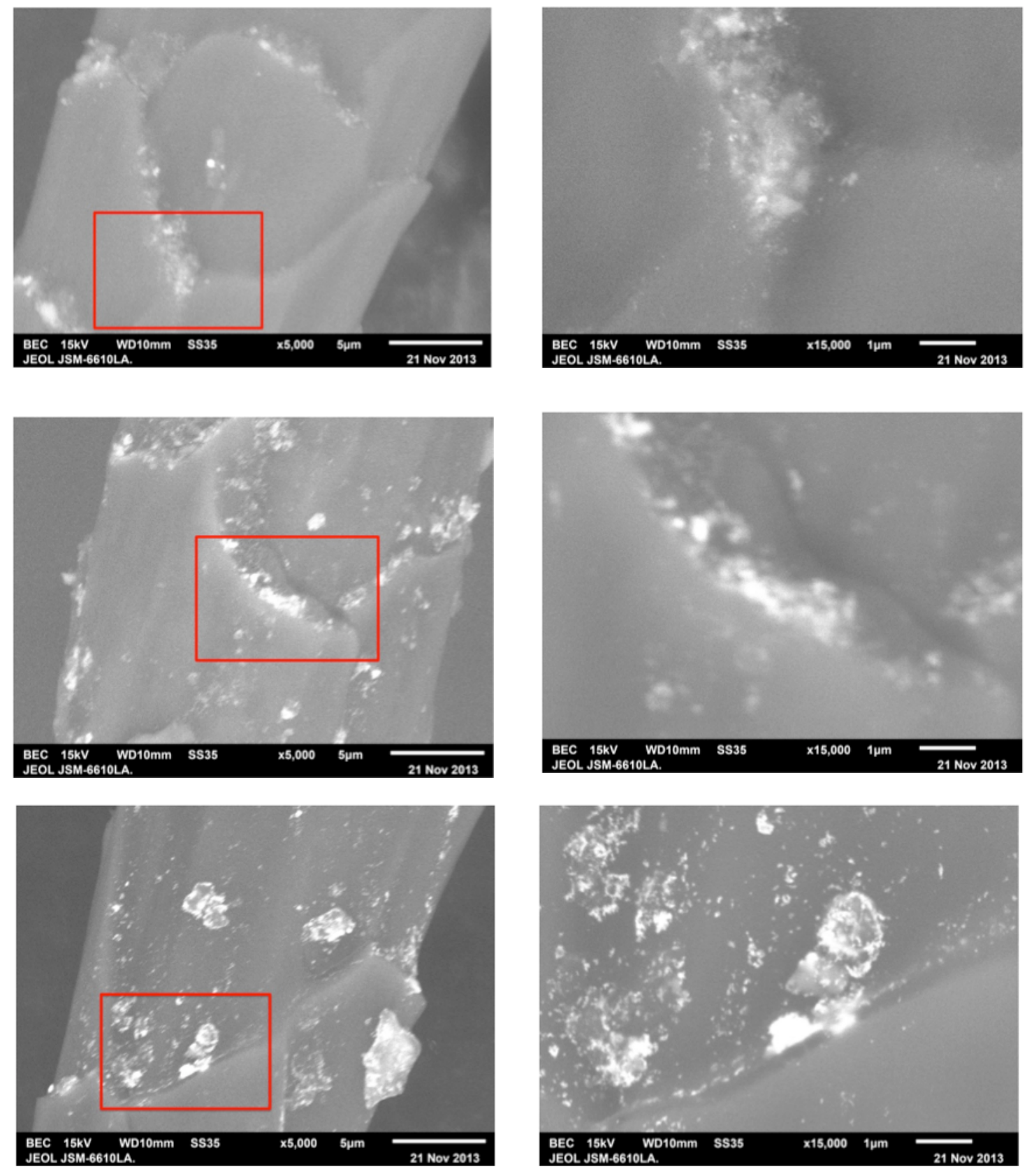

Figure 5.3: SEM images of wool coloured pink (top), purple (middle) and grey (bottom) by spherical gold nanoparticles, at low magnification (left) and high magnification (right, magnified areas shown in red boxes on left images). 


\subsection{Core-shell Particles as a Colourant}

Wool was coloured using silica-gold core-shell particles as synthesised in sample MGN1c, see Section 4.9 Second Adaptations of MGN1 Synthesis. The colloid was washed three times with ethanol by centrifugation and redispersed in water. The $\mathrm{pH}$ of this colloid was lowered and the wool was soaked at room temperature overnight. Some colour remained in the colloid and this was analysed using UV-Vis absorption spectroscopy. The coloured wool was analysed using ColourQuest to obtain CIE Lab values and reflectance data, SEM and XRD. A Photograph of the wool coloured using core-shell particles is shown in Figure 5.4.

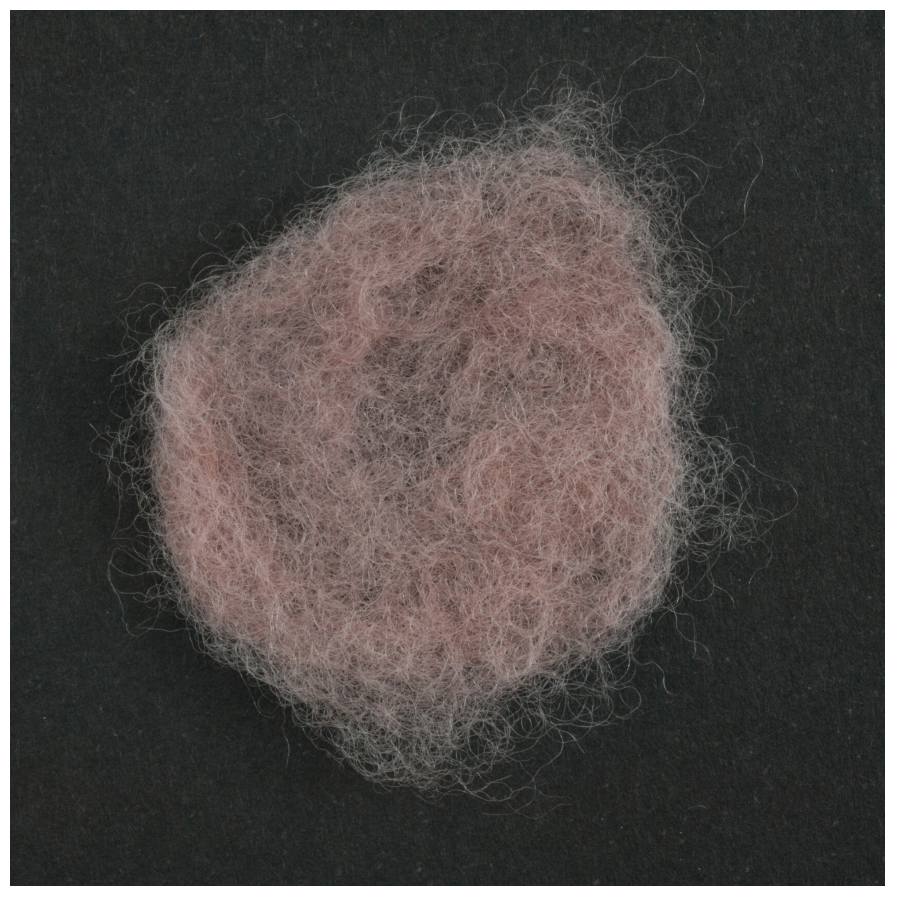

Figure 5.4: Photograph of wool coloured using silica-gold core-shell particles.

\subsubsection{UV-Vis Absorption Spectroscopy}

UV-Vis absorption spectroscopy of the liquid left over after the colouring of the wool showed that some gold nanoparticles were left in the colloid as SPR absorption bands were still exhibited. As seen in Figure 5.5, the UV-Vis absorption 
spectrum shows a major peak at $580 \mathrm{~nm}$ and a minor peak at $710 \mathrm{~nm}$, indicating that the particles left in the colloid were likely to be spherical gold nanoparticles as well as some core-shell particles, respectively. As seen in Figure 5.6, this spectrum shows a peak at $710 \mathrm{~nm}$, which was not seen in the UV-Vis absorption spectrum of MGN1c before the colouring of wool (Figure 5.6). This indicates that peaks at lower wavelengths may have been concealing higher wavelength peaks in previous spectra.

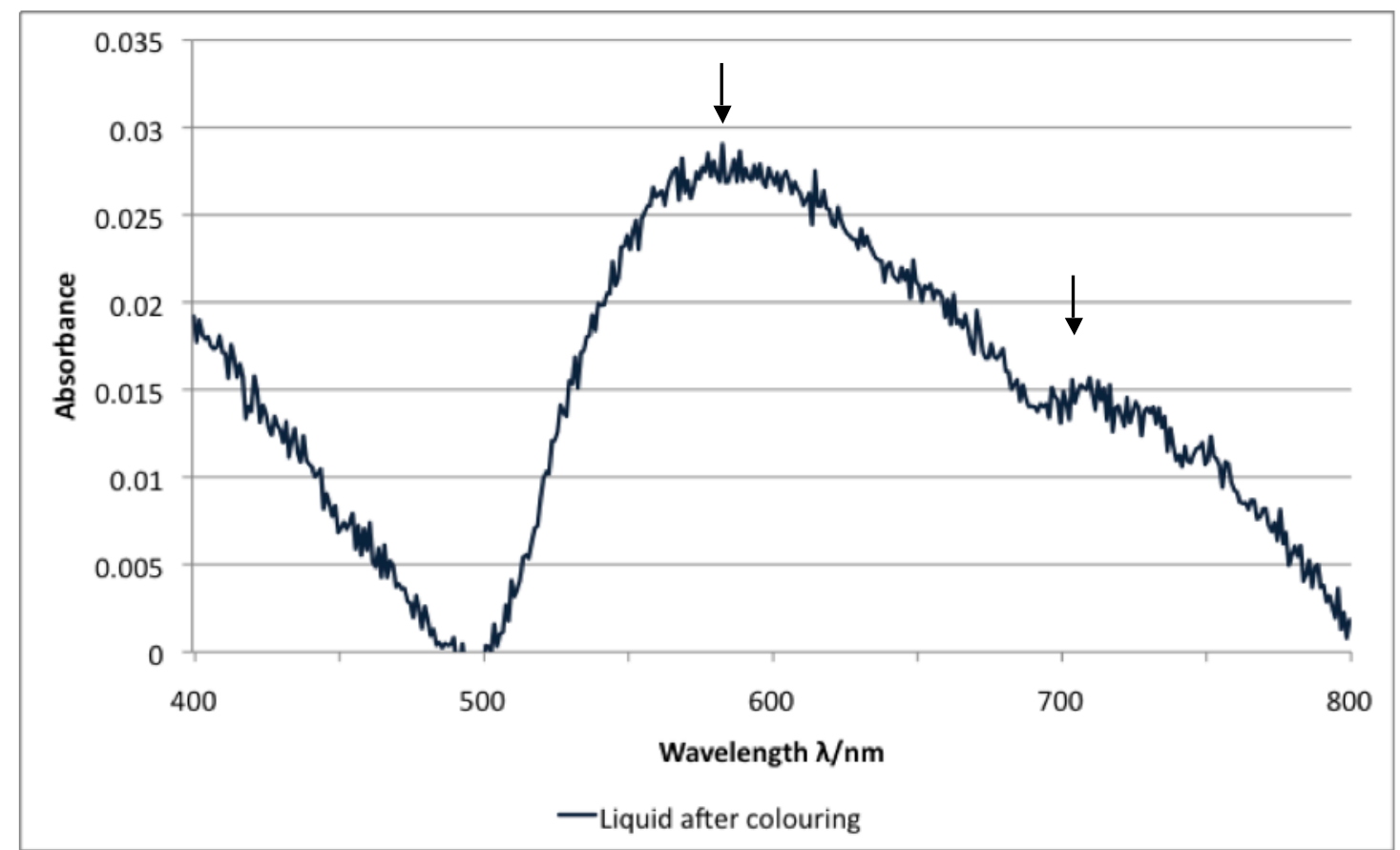

Figure 5.5: UV-Vis absorption spectrum of liquid left over after colouring wool. 


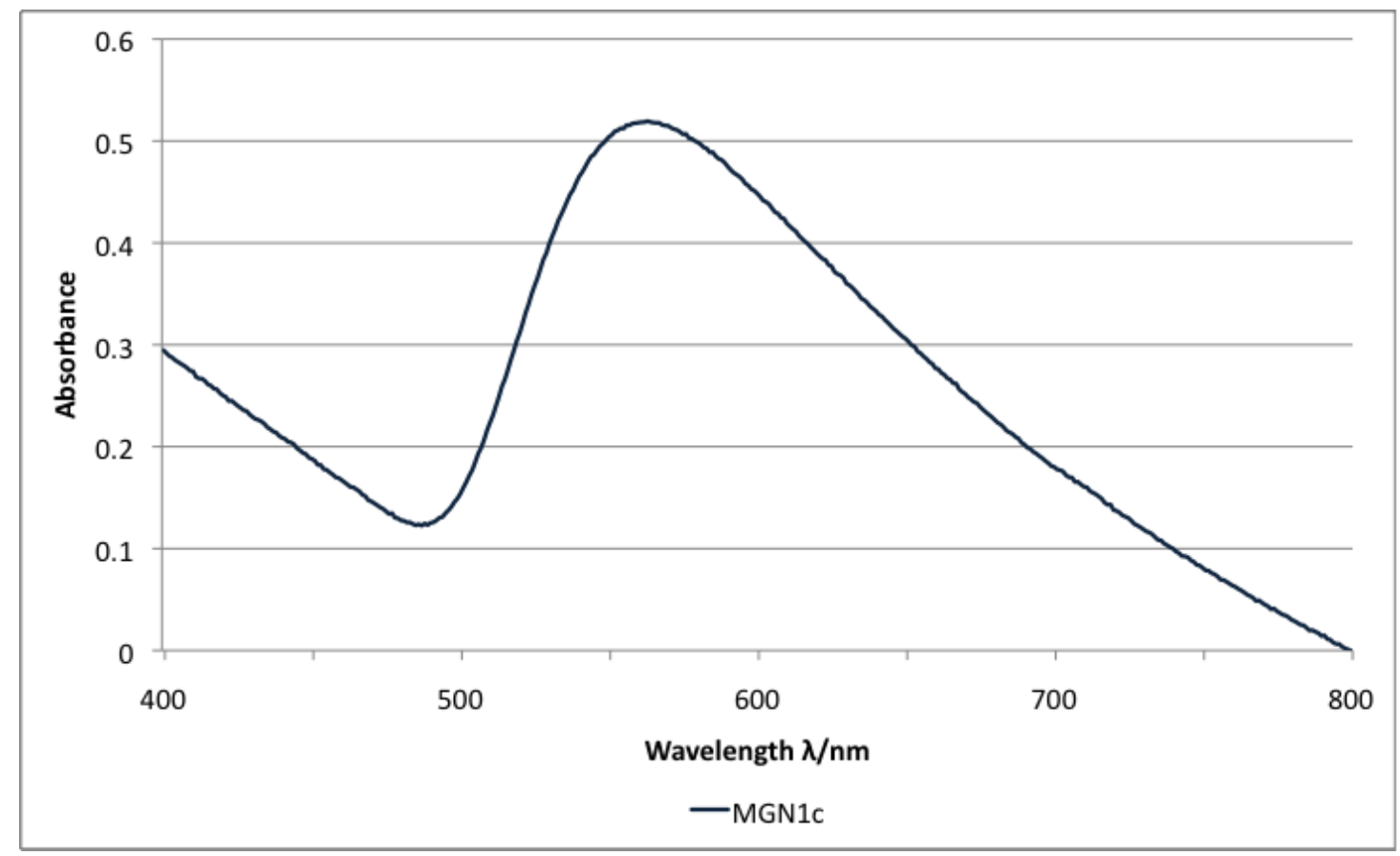

Figure 5.6: UV-Vis absorption spectrum of MGN1c before colouring wool.

\subsubsection{Colour Measurements}

The Kubelka-Munk transformed reflectance spectrum of wool coloured with silica-gold core-shell particles shows SPR characteristics similar to that of the colloid used for its colouring liquor, but it also shows a potential small peak at approximately $700 \mathrm{~nm}$. In the UV-Vis absorption spectrum of MGN1c, there was no observed peak above the peak at $565 \mathrm{~nm}$ attributed to spherical gold nanoparticles, but it is thought that a higher wavelength absorption, indicative of core-shell particles, was being concealed by the broad absorption of the colloid. Here it seems that the broadly absorbing species present in colloid is absent and the small SPR peak of the core-shell particles is observed. 


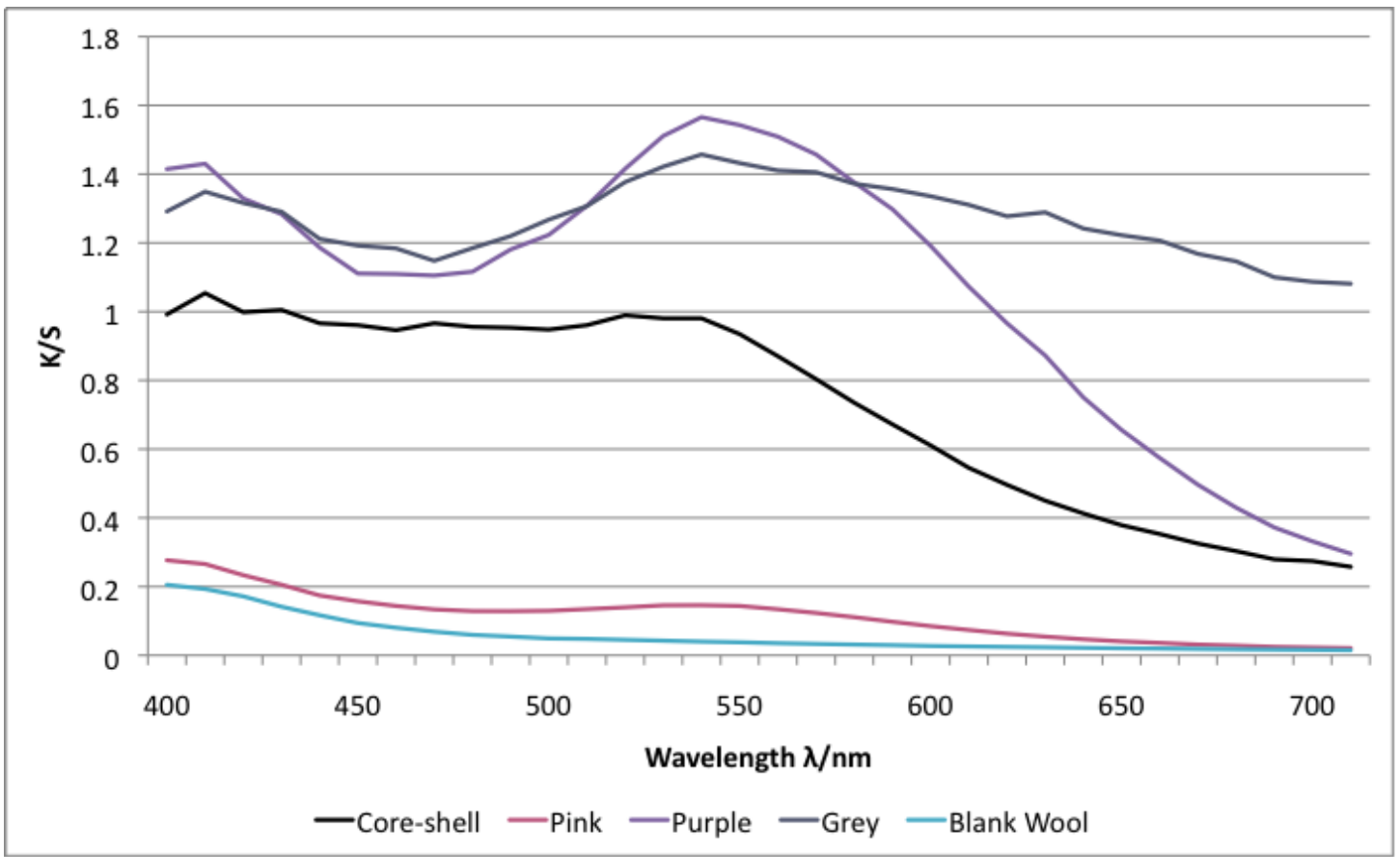

Figure 5.7: Kubelka-Munk transformed reflectance spectrum of wool coloured with core-shell nanoparticles, compared to spectra of wool coloured with spherical gold nanoparticles.

The CIE Lab values of wool coloured with silica-gold core-shell particles are shown in Table 5.2. These values, along with the Kubelka-Munk transformed reflectance spectra confirm that the colour achieved by using silica-gold core-shell particles is different to those achieved by using spherical gold nanoparticles. The CIE Lab values describe the colour with lightness in between that of the pink and purple samples coloured with spherical gold nanoparticles, a more red colour than any achieved with spherical gold nanoparticles and of a very similar yellowness to the pink sample coloured with spherical gold nanoparticles. 
Table 5.2: CIE Lab values of uncoloured wool, wool coloured with spherical gold nanoparticles and wool coloured with silica-gold core-shell particles.

\begin{tabular}{|c|c|c|c|}
\hline Sample & $L^{*}$ value & $a^{*}$ value & $b^{*}$ value \\
\hline Uncoloured & 89.77 & -0.41 & 9.36 \\
\hline Spherical - Pink & 82.78 & 4.17 & 4.51 \\
\hline Spherical - Purple & 54.89 & 6.64 & -2.76 \\
\hline Spherical - Grey & 54.39 & 1.45 & -2.71 \\
\hline Core-shell & 61.81 & 8.67 & 4.39 \\
\hline
\end{tabular}

\subsubsection{SEM}

SEM and EDS were carried out on the JEOL-JSM-6610LA instrument, and samples were coated with $16 \mathrm{~nm}$ of carbon. Again, the wool fibres were observed to be damaged by the electron beam. As seen in Figure 5.8, SEM imaging of wool coloured with silica-gold core-shell particles had many core-shell particles on the fibres. The particles were observed to adsorb preferentially near the cuticle edges of the fibres. This is also seen in the colouring of wool with spherical particles, and is due to the higher Gibbs free energy at these sites. The images show that many particles do tend to adsorb in the same areas with some lone core-shell particles adsorbing in areas far from others. Although many core-shell particles lie very close to others, significant formal aggregation of the particles does not seem to have occurred in these samples, and discrete core-shell particles can be seen.

No spherical gold nanoparticles or aggregates of such were observed under SEM. However, evidence from the transformed reflectance spectrum showed that some spherical particles were likely present. It is assumed that these spherical gold nanoparticles and any potential aggregates of these adsorbed to the fibres along with 
the core-shell particles were too small to observe in SEM images at magnifications used.
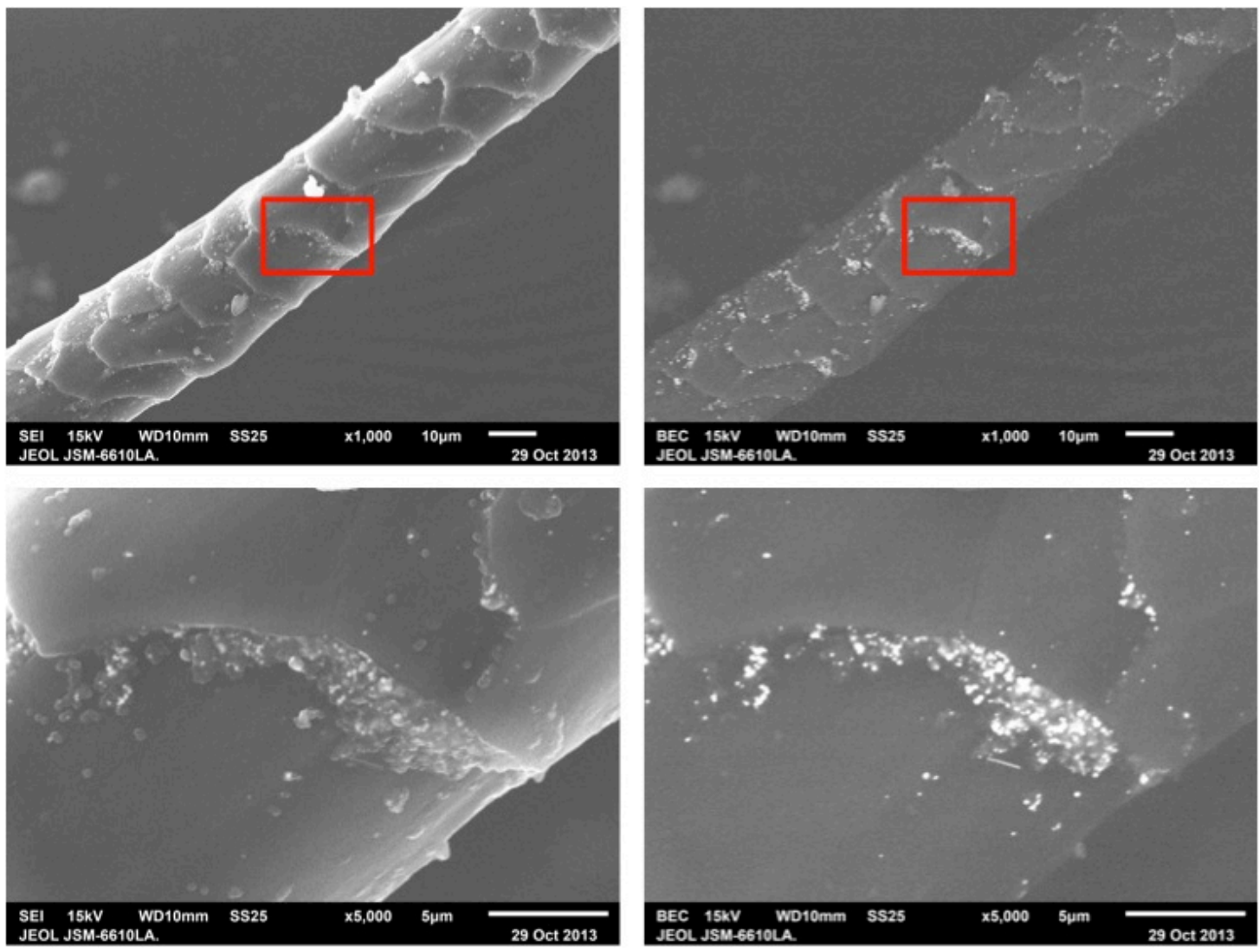

Figure 5.8: SEM images in both SEI (left) and backscatter (right) modes of wool coloured with silica-gold core-shell particles. Red box in low magnification images (top) indicates area shown at higher magnification (bottom).

EDS mapping was carried out to confirm that the areas of high-density material were the core-shell particles, and not aggregates of gold nanoparticles. As seen in Figure 5.9, the EDS map showed silicon in the same areas as gold, confirming that the wool is coloured by core-shell particles. Due to the high oxygen content of the wool fibres, the elemental mapping of oxygen provided no useful information. 

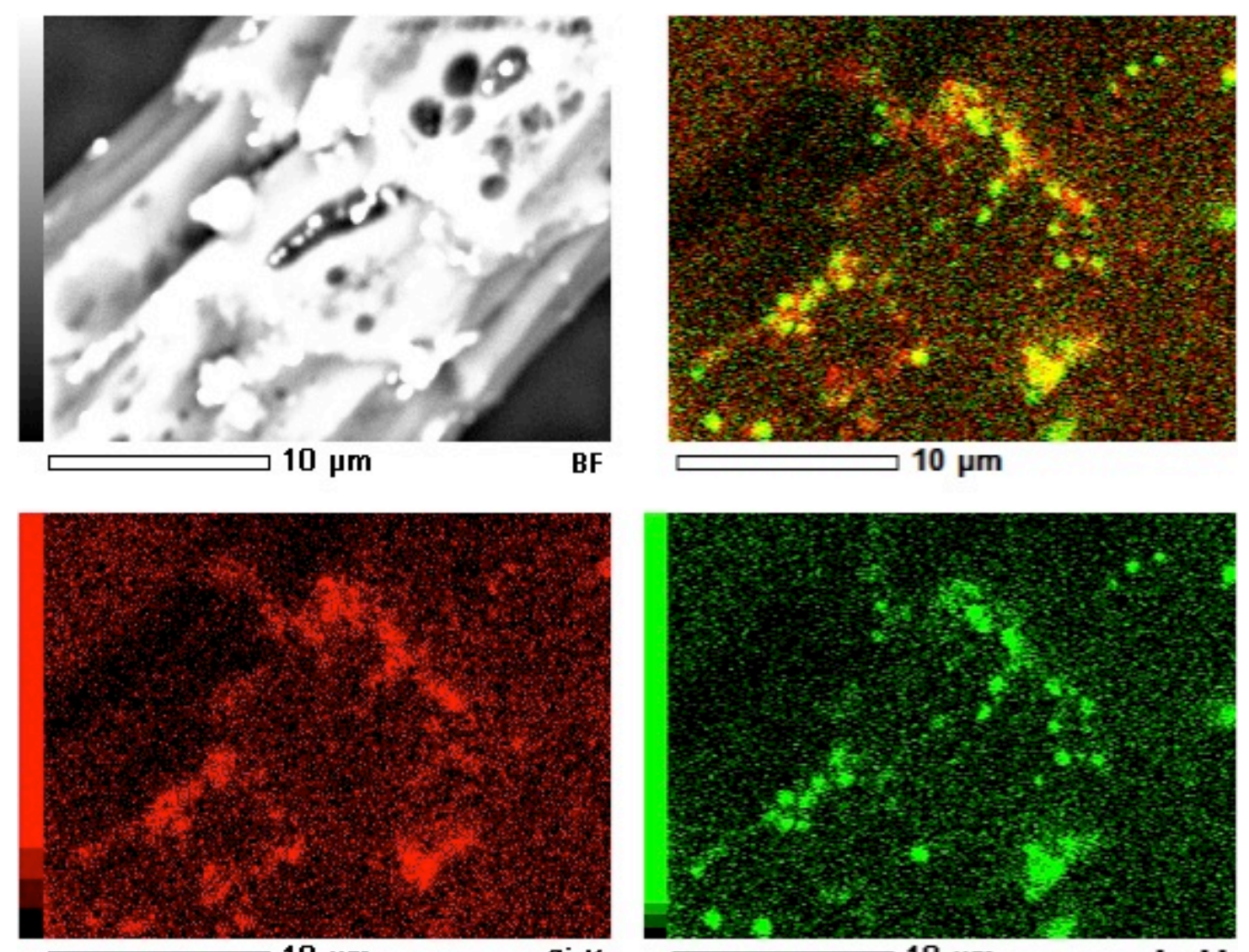

$10 \mu \mathrm{m}$

Si K

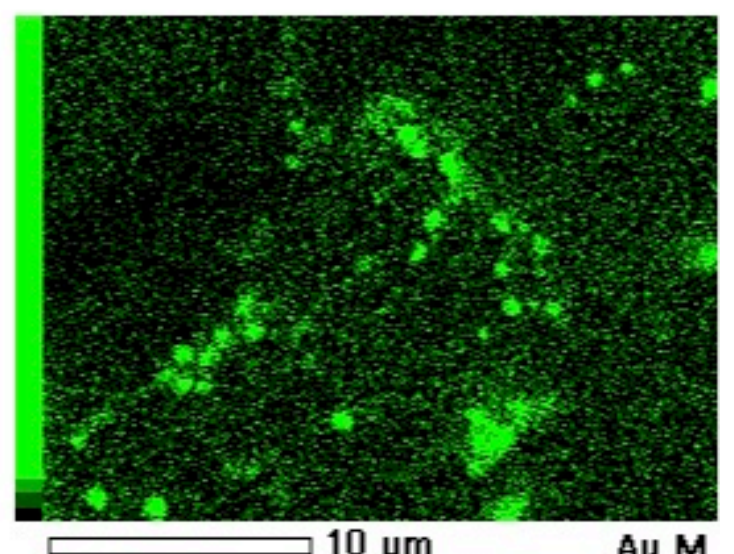

Figure 5.9: EDS map of wool coloured with silica-gold core-shell particles.

Top left: Backscatter image (showing dark areas corresponding to holes in fibre due to beam damage). Bottom left: Silicon map. Bottom right: Gold map.

Top right: Overlay of silicon and gold elemental maps.

\subsection{Conclusions from Colouring of Wool}

Silica-gold core-shell particles as synthesised for sample MGN1c were successfully used to colour wool fibres. The colour achieved with these particles was found to be different to those achieved with spherical gold nanoparticles, and hence successfully fulfilled one of the goals of this research, to increase the achievable colour range of wool using nanogold structures as colourants. 


\section{Conclusions and Future Work}

Capping titania with MPTMS was unsuccessful, it was found that the titania agglomerated and adhered to the walls of the vial. Since these titania spheres could not be capped with MPTMS, gold shells could not be formed on these surfaces. Hence, titania was not used as a core material.

The adapted Stöber method used yielded silica spheres of approximately $180 \mathrm{~nm}$ diameter. These silica spheres were successfully capped with either PEI or MPTMS. It was found that the spheres capped with PEI did not serve as a useful substrate for the growth of gold shells; instead many gold nanoparticles were formed on the PEI functionalised surface. Silica spheres capped with MPTMS were found to be useful cores for the synthesis of gold shells. Many synthetic methods were carried out to manipulate parameters in the gold shell formation leading to many successful syntheses. However, it was found that many MPTMS capped silica spheres remained in the samples, with no gold shells on them. Synthesising samples containing only core-shell particles proved difficult. In fact, even synthesising samples containing a greater number of core-shell particles than silica spheres without gold shells proved challenging. The manipulation of reaction parameters such as increasing the volume of gold used or decreasing the volume of silica used was investigated in an effort to overcome this difficulty. However, it was found that this led to large aggregates of gold in the samples, not associated to the silica surfaces.

Syntheses involving only the capping agent as a reductant and no external reducing agents were found to form some core-shell particles with very thick shells, but the samples were non-uniform so other methods were investigated. The core-shell particles synthesised in this way were observed to have thicker shells than those formed in subsequent syntheses. 
Syntheses involving only hydroxylamine as an external reductant did not form core-shell particles. It was determined that the reducing power of hydroxylamine was insufficient to reduce the amount of gold necessary for a full shell. However, these syntheses did show silica spheres with $\mathrm{AuCl}_{4}{ }^{-}$successfully adsorbed into the MPTMS capping layer indicating that with increased reducing power, the synthesis should yield successful core-shell particles.

Further reactions involving the use of both hydroxylamine and sodium borohydride led to the successful formation of silica-gold core-shell particles. Many reaction conditions were altered to increase the concentration of core-shell particles formed in samples and the synthesis involving a washing step after the addition of sodium borohydride was deemed to be the most successful in achieving this.

Procedures used to colour wool with spherical gold nanoparticles were adapted to colour wool using the silica-gold core-shell particles previously synthesised. These procedures were found to be successful in colouring the wool using these core-shells in the same way as spherical gold nanoparticles.

The colour achieved using silica-gold core-shell particles synthesised in this research to colour wool was shown to be one not previously possible using only spherical gold nanoparticles. This important result fulfilled one of the main aims of this research project.

In the future, investigation into altering the thickness of the gold shells could provide a method for creating additional colours in wool not able to be achieved using spherical gold nanoparticles. In using spherical gold nanoparticles to colour wool, many factors can be used to control the resulting colour. Parameters such as concentration and volume of gold colloid can be manipulated to achieve different colours. The manipulation of similar parameters in the colouring of wool using silica-gold core-shell particles would be another interesting avenue of research into creating new colours in wool using this technology. 


\section{References}

(1) Mie, G. Ann. Phys. 1908, 25, 377.

(2) Turkevich, J.; Stevenson, P. C.; Hillier, J. Disscussions Faraday Soc. 1951, 55.

(3) Pong, B.-K.; Elim, H. I.; Chong, J.-X.; Ji, W.; Trout, B. L.; Lee, J.-Y. J. Phys. Chem. C 2007, 111, 6281 .

(4) Long, N. N.; Vu, L. Van; Kiem, C. D.; Doanh, S. C.; Nguyet, C. T.; Hang, P. T.; Thien, N. D.; Quynh, L. M. J. Phys. Conf. Ser. 2009, 187, 012026.

(5) Demers, L. M.; Mirkin, C. a; Mucic, R. C.; Reynolds, R. a; Letsinger, R. L.; Elghanian, R.; Viswanadham, G. Anal. Chem. 2000, 72, 5535.

(6) Haruta, M. Catal. Today 1997, 861, 153.

(7) Haruta, M.; Yamada, N.; Kobayashi, T.; Iijima, S. J. Catal. 1989, 115, 301.

(8) Haruta, M.; Tsubota, S.; Kobayashi, T.; Kageyama, H.; Genet, M. J.; Delmon, B. J. Catal. 1993, 144, 175.

(9) Haruta, M. Chem. Rec. 2003, 3, 75.

(10) Kelly, K. L.; Coronado, E.; Zhao, L. L.; Schatz, G. C. J. Phys. Chem. B 2003, 107,668 .

(11) Liz-Marzan, L. M. Mater. Today 2004, 7, 26.

(12) Renteria-Tapia, V. M.; Garcia-Macedo, J. J. Nanosci. Nanotechnol. 2008, 8, 6545 .

(13) Link, S.; El-sayed, M. A. J. Phys. Chem. B 1999, 8410.

(14) Murphy, C. J.; Thompson, L. B.; Chernak, D. J.; Yang, J. A.; Sivapalan, S. T.; Boulos, S. P.; Huang, J.; Alkilany, A. M.; Sisco, P. N. Curr. Opin. Colloid Interface Sci. 2011, 16, 128.

(15) Prodan, E.; Radloff, C.; Halas, N. J.; Nordlander, P. Science 2003, 302, 419.

(16) Edgar, J. A. Development of New Methods for the Synthesis of PlasmonicallyActive Precious Metal Rods and Shells, University of Technology, Sydney, 2011.

(17) Oldenburg, S. J.; Averitt, R. D.; Westcott, S. L.; Halas, N. J. Chem. Phys. Lett. 1998, 288, 243.

(18) Prodan, E.; Nordlander, P. Nano Lett. 2003, 3, 543. 
(19) Raschke, G.; Brogl, S.; Susha, A. S.; Rogach, A. L.; Klar, T. A.; Feldmann, J.; Fieres, B.; Petkov, N.; Bein, T.; Nichtl, A.; Kurzinger, K. Nano Lett. 2004, 4, 1853.

(20) Raschke, G.; Brogl, S.; Susha, a. S.; Rogach, a. L.; Klar, T. a.; Feldmann, J.; Fieres, B.; Petkov, N.; Bein, T.; Nichtl, a.; Kürzinger, K. Nano Lett. 2005, 5, 811.

(21) Zhang, J. Z.; Schwartzberg, A. M.; Norman, T.; Grant, C. D.; Liu, J.; Bridges, F.; van Buuren, T. Nano Lett. 2005, 5, 809.

(22) Norman, T. J.; Grant, C. D.; Magana, D.; Zhang, J. Z.; Liu, J.; Cao, D.; Bridges, F.; Van Buuren, A. J. Phys. Chem. B 2002, 106, 7005.

(23) Averitt, R. D.; Sarkar, D.; Halas, N. J. Phys. Rev. Lett. 1997, 78, 4217.

(24) Cortie, M. B.; McDonagh, A. M. Chem. Rev. 2011, 111, 3713.

(25) Schwartzberg, A. M.; Olson, T. Y.; Talley, C. E.; Zhang, J. Z. J. Phys. Chem. B 2006, 110, 19935.

(26) Darwish, M. S. a.; Kunz, U.; Peuker, U. J. Appl. Polym. Sci. 2013, 129, 1806.

(27) Yang, D.; Tian, M.; Wang, W.; Li, D.; Li, R.; Liu, H.; Zhang, L. Electrochim. Acta 2013, 87, 9 .

(28) Zeleòáková, A.; Zeleòák, V.; Degmová, J.; Kováè, J.; Sedláèková, K.; Kusý, M.; Sitek, J. Rev. Adv. Mater. Sci. 2008, 18, 4.

(29) Phonthammachai, N.; Kah, J. C. Y.; Jun, G.; Sheppard, C. J. R.; Olivo, M. C.; Mhaisalkar, S. G.; White, T. J. Langmuir 2008, 24, 5109.

(30) Xue, J. G.; Wang, C. G.; Ma, Z. F. Mater. Chem. Phys. 2007, 105, 419.

(31) Phonthammachai, N.; White, T. J. Langmuir 2007, 23, 11421.

(32) Oldenburg, S. J.; Westcott, S. L.; Averitt, R. D.; Halas, N. J. J. Chem. Phys. 1999, 111, 4729.

(33) Brito-Silva, A. M.; Sobral, R. G.; Barbosa-Silva, R.; de Araujo, C. B.; Galembeck, A.; Brolo, A. G. Langmuir 2013, 29, 4366.

(34) Shi, W. L.; Sahoo, Y.; Swihart, M. T.; Prasad, P. N. Langmuir 2005, 21, 1610.

(35) Xinheng, L.; Masahiko, T. K.; Akira, B.; Hara, M. J. Nanosci. Nanotechnol. 2009, 9, 408.

(36) Kuchibhatla, S.; Karakoti, A. S.; Seal, S. JOM 2005, 57, 52.

(37) http://physchem.kfunigraz.ac.at/sm/Personal/Gerhard/Projects.htm.

(38) Richardson, M. J.; Johnston, J. H. J. Colloid Interface Sci. 2007, 310, 425.

(39) Stober, W.; Fink, A.; Bohn, E. J. Colloid Interface Sci. 1968, 26, 62. 
(40) Carp, O.; Huisman, C. L.; Reller, A. Prog. Solid State Chem. 2004, 32, 33.

(41) Testino, A.; Bellobono, I. R.; Buscaglia, V.; Canevali, C.; D’Arienzo, M.; Polizzi, S.; Scotti, R.; Morazzoni, F. J. Am. Chem. Soc. 2007, 129, 3564.

(42) Yan, M.; Chen, F.; Zhang, J.; Anpo, M. J. Phys. Chem. B 2005, 109, 8673.

(43) Pandikumar, A.; Murugesan, S.; Ramaraj, R. ACS Appl. Mater. Interfaces 2010, 2, 1912.

(44) Pandikumar, A.; Ramaraj, R. Indian J. Chem. 2011, 50A, 1388.

(45) Liu, R.; Sen, A. J. Am. Chem. Soc. 2012, 134, 17505.

(46) Johnston, J. H.; Richardson, M. J.; Burridge, K. A. In Nsti Nanotech 2008, Vol 1, Technical Proceedings; Laudon, M.; Romanowicz, B., Eds.; 2008; pp. 712715 .

(47) Johnston, J. H.; Lucas, K. A. Gold Bull. 2011, 44, 85.

(48) Johnston, J. H.; Burridge, K. A.; Kelly, F. M.; Hendy, S. C.; Brown, I. W. M. In AIP Conference Proceedings; Aip, 2009; pp. 189-192.

(49) Johnston, J. H.; Lucas, K. A. No Title. 5985543, 2012.

(50) Burridge, K. Gold and Silver Nanoparticles as High-value Colourants and Multi-functional Entities for Natural Fibres and Minerals, Victoria University of Wellington, 2010, Vol. PhD.

(51) Simpson, W. S.; Crawshaw, G. H. Wool: Science and Technology; McIntyre, P. J. E., Ed.; CRC Press LLC and Woodhead Publishing Ltd, 2002.

(52) Brown, K. R.; Natan, M. J. Langmuir 1998, 14, 726.

(53) Richardson, M. J.; Johnston, J. H.; Borrmann, T. Eur. J. Inorg. Chem. 2006, 2618.

(54) Meeks, N. D.; Rankin, S.; Bhattacharyya, D. Ind. Eng. Chem. Res. 2010, 49, 4687.

(55) Dohi, O.; Yagi, N.; Wada, T.; Yamada, N.; Bito, N.; Yamada, S.; Gen, Y.; Yoshida, N.; Uchiyama, K.; Ishikawa, T.; Takagi, T.; Handa, O.; Konishi, H.; Wakabayashi, N.; Kokura, S.; Naito, Y.; Yoshikawa, T. Digestion 2012, 86, 161.

(56) Freestone, I.; Meeks, N.; Sax, M.; Higgitt, C. Gold Bull. 2007, 40, 270.

(57) Lin, C.; Tao, K.; Hua, D.; Ma, Z.; Zhou, S. Molecules 2013, 18, 12609. 